\title{
Villes du Sahara
}

\section{Urbanisation et urbanité dans le Fezzan libyen}

Pliez, O. (2003). Villes du Sahara: urbanisation et urbanité dans le Fezzan libyen. CNRS.

http://books.openedition.org/editionscnrs/3729 


\section{REMERCIEMENTS}

Mes remerciements vont d'abord à Marc Cote, Professeur émérite à l'Université de Provence, pour sa patience, son soutien et ses conseils.

Toute ma reconnaissance va aussi aux membres de la Section $39 \mathrm{du}$ CNRS, qui en m'accordant un détachement, m'ont offert la possibilité de mener ce travail dans les meilleures conditions.

C'est aux membres de l'IREMAM, mon unité d'accueil, que vont ensuite mes remerciements. A son directeur, Christian Robin, Directeur de recherche au CNRS. A Jean-Robert Henry, Directeur de recherche, et Jean-Luc Arnaud, Chargé de recherche, qui ont accepté de m'intégrer dans leurs programmes de recherches.

Mes travaux n'auraient pas pris le même tour sans les conseils, les informations et l'aide d'André Laronde, Professeur à Paris IV, Taoufiq Monastiri, Ingénieur de recherche au CNRS, à la MMSH, Jacques Fontaine, Maître de conférences à Besançon, Hélène Claudot-Hawad, Directrice de recherche au CNRS, Tahar Najjar et Mounir Benhamida et à Tripoli, Mohamed Jerary et Mahmoud ad Deek, du Libyan Studies Center, Ahmed Fawzi Hellal, Directeur du centre national de cartographie, M. Karmous du centre des statistiques et Benoît Deslandes, attaché culturel à l'Ambassade de France.

Un travail de recherche, c'est aussi la rencontre avec ceux qui m'ont permis de mieux comprendre ce que vivre dans le Sahara peut signifier, plus particulièrement Jamal et sa famille à Sebha, Farouk et Azeledin à Takarkiba, Ali à Al Gharayfa, Ahmed Abdalla à Sebha et Ali Smail à Oubari mais aussi Anne Cote, et Yassin et Sassia Spiga, compagnons de terrain.

Je remercie Nathalie et Vanessa d'avoir accepté la pénible tâche de relecture de ce travail.

A Laurence, Salomé et Pénélope. 


\section{UNE LECTURE URBAINE DU FEZZAN}

Parler du Sahara conduit rapidement à se heurter à la représentation que l'on se fait des déserts. Les «beaux livres » de photographies ou les brochures d'agences de voyage médiatisent des images attendues, celles d'infinités vides, de nomades qui scrutent ces paysages en buvant du thé vert, d'agriculteurs sédentaires passés maîtres dans l'art du ciselage des jardins d'oasis ou des techniques séculaires d'irrigation.

Vu de loin, au prisme de l'imaginaire, des récits de découverte ou des belles études géographiques menées de l'Entre-deux-guerres aux années $1960^{1}$, le Fezzan, cette vaste région du Sahara libyen longtemps à la croisée de grandes routes caravanières historiques, réunissait a priori les ingrédients de ce que l'on imagine être encore le Sahara. Pourtant, dans cet espace que l'on serait un peu vite tenté de classer parmi les «confins » dont la science sociale coloniale était si friande, point de «civilisation paysanne » similaire à celles que de nombreux travaux ont tenté de circonscrire dans l'ensemble du Maghreb, point non plus de laborieux agriculteurs travaillant ces jardins comme le révélait le piteux état des palmeraies. Au contraire, plusieurs missions, étalées sur trois années, ont révélé l'ampleur du décalage entre l'image que j'avais de cette région, le Fezzan, et ce qui constitue réellement le quotidien de ceux que l'on appelle encore parfois les Fezzanais.

Il faut donc se rendre à l'évidence et constater que le Sahara contemporain est d'abord urbain. Le désert est parsemé de villes où se concentre la majeure partie des populations. Alors pourquoi les photographes ne nous donnent-ils pas à voir ces villes du Sahara ? Sans doute parce que leur objectif, légitime, consiste à vendre du rêve sur papier glacé... Or les paysages urbains sahariens suscitent plutôt la consternation dans le regard des observateurs extérieurs. On ne peut évidemment pas en rester là mais pour appréhender les mutations sociales et spatiales qu'a connu le Sahara durant ces trois dernières décennies, il est nécessaire d'effectuer un retour sur l'ampleur du processus d'urbanisation au Sahara, les grilles de lecture héritées et celles permettant de comprendre ce que signifie l'urbain dans un espace où l'on ne s'attend a priori pas à le rencontrer.

${ }^{1}$ Citons, sans prétention à l'exhaustivité, H. Duveyrier (1864), J. Despois (1946), J. Lethielleux (1948) et L. Eldblom (1968). 


\section{VERS L'URBANISATION TOTALE DU SAHARA}

\section{UN ÉTAT DES LIEUX}

Le processus d'urbanisation au Sahara frappe par son ampleur, sa sélectivité spatiale et sa rapidité ${ }^{2}$.

La population saharienne, dans les limites du Sahara «français » ${ }^{3}$, est estimée à 1,7 millions d'habitants en 1948, 2 millions en 1966 et 10 millions au milieu des années $1990^{4}$. La croissance démographique est donc récente puisque entre 1948 et 1966 l'importance de l'émigration annulait les effets du croît naturel alors que quarante ans plus tard le Sahara compte cinq fois plus d'habitants. Pour autant, le plus vaste désert du monde compte moins d'habitants que la seule agglomération du Caire.

Le Sahara, peu peuplé, l'est aussi inégalement puisque la moitié des Sahariens vivent le long des marges du désert. Par ailleurs, la croissance démographique contribue à différencier les versants maghrébin, au Nord et sahélien, au Sud. En effet, alors que les régions sahariennes des Etats du Sahel perdent des habitants, les régions les plus méridionales de Libye et d'Algérie, dans le Sahara central, dont les densités sont de l'ordre de 0,5 habitants au $\mathrm{km}^{2}$, connaissent des taux de croissance démographique très élevés, supérieurs à $4 \%$ l'an.

Tableau 1 : Les villes sahariennes de plus de 10000 habitants (mi-1990)

(dans les limites de l'aire définie par Capot-Rey, soit zone saharo-steppique non comprise)

\begin{tabular}{|c|l|c|c|c|c|c|}
\hline & Pays & $\begin{array}{c}\mathbf{1 0 0 0 0} \text { à } \\
\mathbf{2 5 0 0 0} \\
\text { hab. }\end{array}$ & $\begin{array}{c}\mathbf{2 5 0 0 0} \mathbf{5 0} \\
\mathbf{5 0 0 0} \\
\text { hab. }\end{array}$ & $\begin{array}{c}\mathbf{5 0 0 0 0} \text { à } \\
\mathbf{1 0 0 0 0 0} \\
\text { hab. }\end{array}$ & $\begin{array}{c}\mathbf{1 0 0 0 0 0} \\
\text { hab. } \\
\text { et plus }\end{array}$ & Total \\
\hline \multirow{2}{*}{$\begin{array}{c}\text { Nord } \\
\text { (Maghreb) }\end{array}$} & Maroc & 4 & 2 & 1 & 1 & 8 \\
\cline { 2 - 7 } & Algérie & 12 & 8 & 3 & 3 & 26 \\
\hline
\end{tabular}

\footnotetext{
${ }^{2}$ L'état des lieux le plus récent sur l'ensemble de l'aire est celui de Jacques Fontaine (1996).

${ }^{3}$ C'est-à-dire l'ensemble sous domination coloniale française comprenant le Maroc, l'Algérie, la Tunisie, la Mauritanie, le Mali, le Niger et le Tchad, soit une superficie de 4,3 millions de $\mathrm{km}^{2}$.

${ }^{4}$ Nous utilisons comme limite du Sahara l'isohyète $100 \mathrm{~mm}$, choix discutable mais qui permet d'écarter les zones semi-arides des marges, dont l'organisation spatiale s'assimile de plus en plus à celle des foyers de peuplement hors Sahara.
} 


\begin{tabular}{|c|l|c|c|c|c|c|}
\hline & Libye & 10 & 1 & 2 & 1 & 14 \\
\hline \multirow{3}{*}{$\begin{array}{c}\text { Sud } \\
\text { (Sahel) }\end{array}$} & Mauritanie & 2 & 1 & 1 & - & 4 \\
\cline { 2 - 7 } & Niger & 1 & 1 & 2 & - & 4 \\
\cline { 2 - 7 } & Tchad-Mali & - & - & - & - & 0 \\
\hline \multicolumn{2}{|c|}{ Total } & 33 & 14 & 9 & 5 & 61 \\
\hline
\end{tabular}

Source : J. Fontaine, 1996

Ces disparités posent question quant aux ressorts de la croissance d'autant plus que le déséquilibre Nord-Sud est tout aussi urbain que démographique. On compte 53 villes dans le Sahara maghrébin contre 8 dans le Sahara sahélien et si à l'échelle du désert le nombre de villes se réduit au fur et à mesure que l'on monte dans la hiérarchie urbaine, en revanche, à l'échelle des Etats, les situations sont très différentes entre le Tchad et le Mali, sans villes sahariennes de plus de 10000 habitants et le Maroc, l'Algérie et la Libye où certaines des agglomérations dépassent désormais 100000 habitants, paradoxalement situées loin des marges. L'urbanisation saharienne ne relève d'évidence pas d'un mouvement spontané mais de choix d'aménagements nationaux.

Pour autant, ce premier niveau de lecture s'applique surtout aux villes de plus de 10000 habitants, alors que le fait spatial le plus marquant de ces dernières années, commun à tous les Etats et extrêmement rapide, est la multiplication des petites agglomérations. Partout, une véritable « micro-urbanisation » saharienne accompagne la fixation des nomades, la création de centres administratifs et les regroupements forcés de populations. Le phénomène, massif et donc complexe, concerne tout le Sahara.

Le processus d'urbanisation au Sahara est donc caractérisé par l'agglomération généralisée des populations, le clivage entre versants maghrébin et sahélien, le déséquilibre systématique des hiérarchies urbaines, la présence sélective de quelques grandes villes et surtout la multiplication des plus petites. Etablir des parallèles entre ce processus et celui qu'ont connu d'autres régions du monde n'est pas chose aisée ; le Sahara, et sans doute aussi par extension d'autres espaces arides, présentent des logiques qui diffèrent de celles communément admises. C'est donc une grille de lecture que nous tenterons d'élaborer au fil de cette recherche, fondée sur les questions que posent les terrains sahariens et sur les «évidences » que nous venons d'énumérer ; questions empiriques donc, mais dans un cadre plus large dont il convient de définir les contours spatiaux et thématiques. 
Dans ce contexte, l'étude d'une région comme celle du Fezzan apporte un éclairage à une lecture plus générale des changements socio-spatiaux dans le Sahara contemporain ${ }^{5}$.

La localisation du Fezzan est exceptionnelle. Eloigné de près de 700 kilomètres du littoral libyen, dont la capitale, Tripoli, est déjà à la latitude du Bas Sahara algérien, il fait partie du Sahara central ; frontalier de l'Algérie, vers Tamanghasset, du Niger, vers Agadès et du Tchad, vers Bardaï et le Tibesti, espace de contacts entre trois grands groupes ethniques sahariens, les Arabes, les Touaregs et les Toubous, il bénéficie depuis le Moyen-Age d'un privilège de situation que les commerçants arabes et sahéliens ont pérennisé jusqu'aux premières décennies du $X X^{\mathrm{ème}}$ siècle.

Le Fezzan est, par ailleurs, libyen, un cadre géographique incontournable mais hélas très peu investi par les recherches en sciences sociales. La Libye pâtit de la longue parenthèse due aux tensions géopolitiques qui fait que peu de travaux de terrain ont pu y être menés depuis les années 1970. Bien heureusement, le contexte politique change mais laisse les chercheurs sans l'assise théorique nécessaire à mener une recherche de fond dans ce cadre national. Le « cas libyen » est pourtant à plusieurs titres exemplaire, pays saharien, rentier, d'immigration, dont le médiatique dirigeant, Mouammar Kadhafi, a mené avec force un projet social et spatial ambitieux qui a transformé en deux décennies l'ensemble du territoire national.

Le Fezzan est, enfin, l'une des régions les plus peuplées du Sahara, 350 000 habitants, et l'une des plus urbanisées, à près de $90 \%$. Cette urbanisation généralisée constitue une incontestable originalité qui préfigure le devenir de la plupart des régions sahariennes mais dont l'étude permet peut-être aussi d'éclairer le devenir urbain d'espaces sis hors des déserts. Deux modalités de peuplement dominent, largement répandues dans l'ensemble du désert. D'une part les grandes villes, et Sebha, avec près de 120000 habitants, à la fois centre administratif et ville cosmopolite d'un Sahara parcouru par des migrants venant de toute l'Afrique noire en est l'un des exemples les plus éclairants. D'autre part, trois vallées, densément peuplées, dont les habitants vivent dans des villages et des bourgs alignés le long de rubans de palmeraie d'une centaine de kilomètres de long chacun lesquels constituent de fins observatoires de la micro-urbanisation qui caractérise désormais tout le désert.

\footnotetext{
${ }^{5}$ Afin de ne pas multiplier les croquis, je renvoie le lecteur à la présentation détaillée du Fezzan dans le Chapitre 1 de la Première partie.
} 
Une recherche sur le Fezzan permet donc d'enrichir trois champs de connaissances, ceux de la Libye, du Sahara et des nouvelles dynamiques de l'urbain.

Deux fils directeurs fondent la lecture des mutations socio-spatiales du Sahara contemporain. La première a trait aux modalités selon lesquelles a été mené le processus d'urbanisation, commodément imagé par la dichotomie «urbanisation du haut vers le bas et du bas vers le haut». Derrière cette formule, se profile toute la question des rapports de l'Etat au processus urbain mais aussi aux espaces et aux sociétés sahariens. Le second concerne l'articulation entre l'urbanisation et les réseaux. Des systèmes sont construits, commerçants ou étatiques, dans lesquels les villes ont des fonctions différentes selon le type de réseau dans lequel elles sont insérées, qu'il s'agisse du commerce transsaharien ou de la construction du territoire national. C'est donc à l'épaisseur historique de ce rapport qu'il faut se référer afin de donner sens à une urbanisation qui semble surgir ex-nihilo.

\section{URBANISATION PAR LE HAUT, URBANISATION PAR LE BAS}

La distinction proposée par Gilles Sautter (1982) entre l'urbanisation du haut en bas et l'urbanisation remontante illustre bien les modalités du processus d'urbanisation dans l'ensemble du Sahara maghrébin. Pour autant, le «cas libyen » est singulier car la masse des moyens financiers dont a disposé l'Etat afin de mener cette politique en fait à bien des égards un cas d'école de l'urbanisation de haut en bas, menée par un Etat rentier, plus proche en cela du cas saoudien que des autres Etats sahariens. Plus que dans le reste du Maghreb, les autorités politiques libyennes ont eu les moyens de leurs ambitions, appliquant une politique d'urbanisation systématique sur l'ensemble du désert, mais ce processus s'est heurté à plusieurs obstacles, la chute des cours du baril de pétrole, une crise économique durable et l'ostracisme international qu'a finalisé la mise sous embargo de 1992. En conséquence, la Libye représente aussi un exemple intéressant de la manière dont le mouvement inverse d'urbanisation a été impulsé, du bas vers le haut, en réponse à des dynamiques locales fortes mais surtout, parce que le désengagement de l'Etat imposait aux sociétés sahariennes de prendre en charge l'héritage récent que leur laissaient deux décennies d'intervention massive de l'échelon national vers l'échelon local.

Le manichéisme sous-jacent à cette lecture du processus d'urbanisation en deux temps mérite cependant d'être nuancé, car en fin de compte, aucun 
des deux mouvements n'agit indépendamment l'un de l'autre; en revanche, ils varient en intensité selon les lieux et les périodes. La question de «l'après Etat », comme acteur largement prépondérant, n'en est donc que plus essentielle puisque l'Etat ne s'est substitué qu'un temps au « retour du local» (P.R. Baduel, 1985, M. Lavergne, 1995) longtemps différé mais aujourd'hui actif et multiforme. Alors que la logique d'Etat a, durant deux décennies, promu un modèle égalitariste d' " urbanisation des campagnes », aujourd'hui, les espaces sahariens se transforment sous l'impulsion de nouveaux ressorts et selon de nouvelles logiques d'action. Les pratiques habitantes, par exemple, révèlent que l'aspect rural n'est plus le déterminant majeur du Fezzan mais bien l'une des manifestations de son identité urbaine.

La petite ville survivra-t-elle ? se demandaient Saad Qezeiri et Richard Lawless en 1986. Disposant de revenus considérables et dans un contexte de croissance démographique et de migrations intenses, l'Etat libyen a créé les petites villes tout en les rendant dépendantes de ses subsides, eux-mêmes indexés sur les cours du baril de pétrole. Or cette politique fut impulsée alors que les cours augmentaient rapidement et tout laissait supposer que cette urbanisation de la croissance deviendrait une urbanisation de la crise avec la chute des cours. En somme, la baisse des revenus de l'Etat aurait révélé le caractère artificiel de cette urbanisation, «simple épisode dans la longue évolution du système de peuplement libyen ». Cette conclusion pessimiste ne surprend pas rétrospectivement, tant on peut être frappé par la massiveté de l'intervention de l'Etat. Dans une large mesure, elle a différé la dynamique inverse de « retour du local » repérée dans l'ensemble du Monde arabe durant les années 1980. Mais une quinzaine d'années après les analyses de Saad Qezeiri et Richard Lawless, nombre de petites villes des années 1960 et 1970 sont désormais des agglomérations de rang supérieur, intégrées dans une hiérarchie urbaine qui ne cesse de s'étoffer. Par ailleurs, la dynamique se poursuit malgré le contexte de crise économique et politique, laissant supposer que la petite ville a non seulement survécu mais qu'elle devient même au Sahara le mode de peuplement dominant.

Il convient donc de distinguer la crise de l'agglomération et les dynamiques urbaines car il existe un décalage entre ce que l'Etat a créé et les évolutions par rapport à ce support. C'est ainsi que d'un côté, la pénurie en logements ou les problèmes d'assainissement s'inscrivent dans les paysages et marquent le quotidien des habitants tandis que de l'autre, les migrations et de nouveaux repères identitaires contribuent au développement rapide d'une urbanité qui remet en cause le découpage ville-campagne. $\mathrm{La}$ rapidité des changements a pour 
conséquence l'absence de coupure nette, inscrite dans la durée, entre une ville et «sa » campagne car pendant que la ville émergeait, les campagnes connaissaient des mutations tout aussi rapides. Le continuum urbain n'est donc pas ici une dynamique qui transcenderait la coupure traditionnelle entre rural et urbain mais une évidence qu'il convient de préciser dans ses modalités et ses caractères.

\section{LES RÉSEAUX DE CIRCULATION PRODUISENT DE L'URB AIN}

On aura compris que le « retour du local» ne peut être réduit au seul affaiblissement de l'Etat. Il est révélateur, autant que produit, du système relationnel dense dans lequel est inséré le Fezzan, visible aux échelles régionales, nationales et sahariennes. Ceci est particulièrement net lorsque l'on prend la mesure des réseaux urbains actuels, véritables pivots d'une intégration régionale en cours. Le dynamisme des villes situées dans le Sahara libyen doit aujourd'hui moins qu'on ne pourrait le supposer a priori à l'effet des politiques d'intégration nationale et plus à un rôle de marge entre deux aires culturelles. On prend en effet sans doute à peine la mesure de l'intensité avec laquelle s'effectue actuellement la réactivation du réseau saharien. Une intensité qui rime souvent avec fragilité, due aux géopolitiques qui se jouent dans ces « confins saharo-sahéliens » où, au gré des opportunités, la circulation s'établit de part et d'autre des frontières entre la Libye, l'Algérie, le Tchad et le Niger, s'appuyant sur un ensemble de relais et de carrefours qui redessinent la trame urbaine du Sahara.

Le «cas libyen» révèle donc une dimension que l'on croyait totalement disparue dans le Sahara maghrébin en voie d'intégration aux Etats nationaux, celle de la circulation dans des espaces transnationaux. Ainsi se profile un fil directeur fondamental pour comprendre le Sahara libyen contemporain dans toute sa dimension historique, l'étroite relation entre les agglomérations et les réseaux dans lesquels elles sont insérées.

L'approche diachronique des relations entre réseaux et agglomérations au Sahara maghrébin (fig.1) peut être décomposée en quatre étapes chronologiques mais non datées pour la simple raison que les modalités diffèrent fortement selon les régions et les Etats. Quelques lieux-clés reviennent : le Nord, entendu ici au sens de littoral et de lieu de pouvoir où se décide le devenir du Sahara. L'organisation spatiale des quatre Etats maghrébins se décline selon un gradient Nord-Sud, 
Figure 1 : Urbanisation et circulations : une approche diachronique

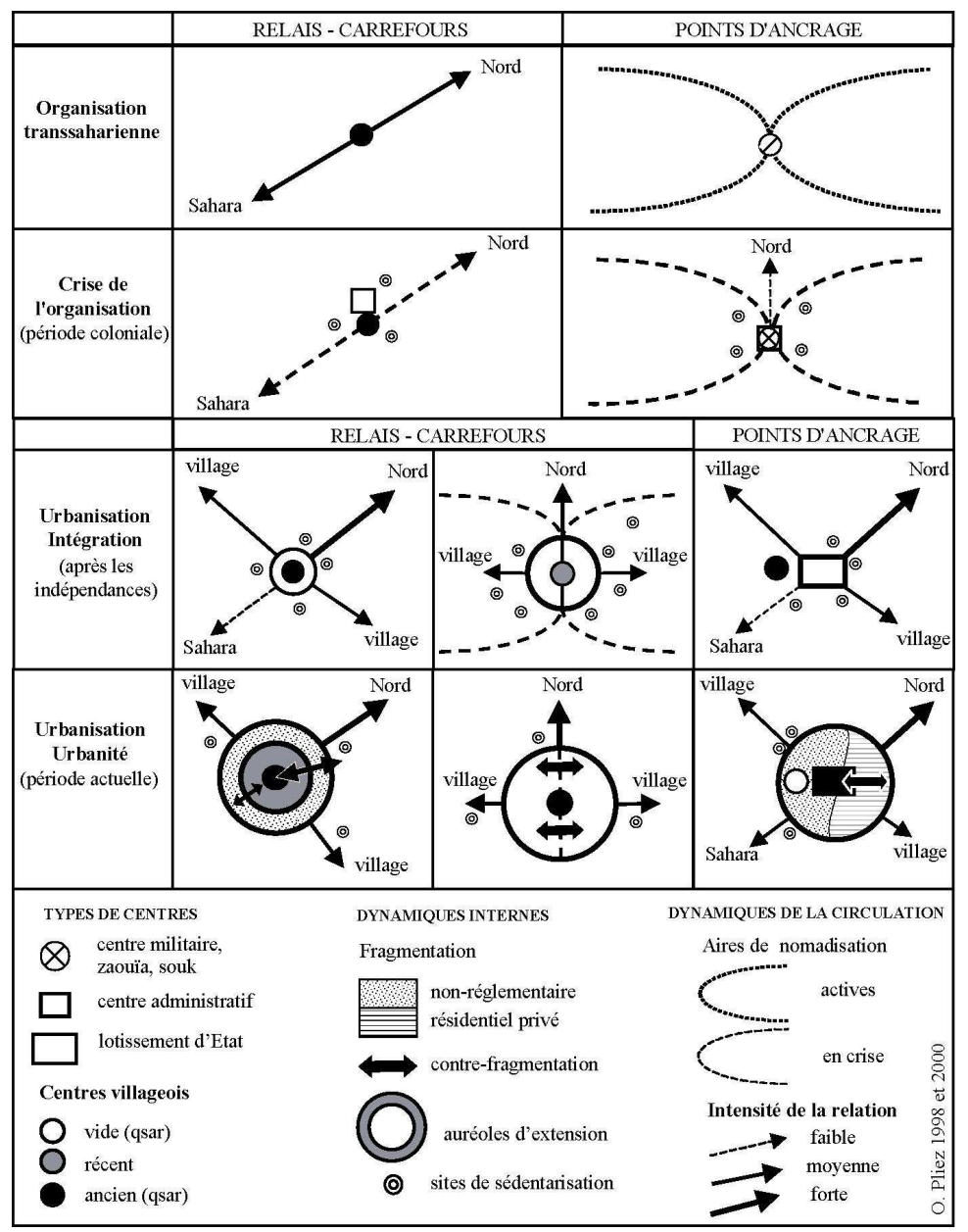


littoral/montagnes/désert de densités et d'activités décroissant dont l'intensité varie selon les spécificités nationales de chaque système spatial; le Sahara est le second lieu-clé, espace parcouru ou cloisonné selon les périodes et donc selon le rôle qui lui est imparti par le Nord. Les types de centres sont les noyaux initiaux de peuplement, ksour (sing. ksar), villages généralement fortifiés, centres militaires coloniaux, zaouïas (centres confrériques) ou souks (marchés) et plus récemment centres administratifs ou lotissements d'Etat lorsque les centres administratifs sont doublés de programmes d'habitation. Les types de centres se transforment au fil des quatre périodes par des dynamiques internes d'extension, de sédentarisation ${ }^{6}$ et de fragmentation ou de contre-fragmentation selon que la tendance est plutôt à la segmentation sociale ou à la porosité entre les différents quartiers qui composent les agglomérations. Les dynamiques de la circulation ont un effet déterminant sur la transformation des agglomérations qui rend nécessaire d'en distinguer les modalités et l'intensité.

L'organisation transsaharienne se caractérise par la valorisation de la fonction de circulation. Les acteurs essentiels en sont les commerçants et les nomades. Les sites se répartissent en deux catégories, soit des oasis-relais dynamiques situées sur les grandes routes caravanières dépendantes de ceux qui circulent (ou en déclin si elles ne le sont plus), soit des points d'ancrage, puits, zaouïa, souk, situés à la convergence d'aires de parcours pastorales différentes. Dans les deux cas, le système spatial se fonde sur le réseau comme principe organisateur essentiel.

La crise de l'organisation débute à la fin du XIX $\mathrm{X}^{\mathrm{èm}}$ siècle, sous l'impulsion des Ottomans et des colonisateurs européens pour s'achever progressivement après les indépendances. Partout la volonté de contrôler les territoires conduit les autorités militaires à implanter des infrastructures administratives, militaires ou

\footnotetext{
${ }^{6}$ Le terme de sédentarisation, utilisé ici par commodité et pour son caractère évocateur, sera remplacé dans le corps du texte par l'expression «fixation des nomades » afin d'éviter l'équivoque qui conduirait à supposer que les nomades deviennent sédentaires en se fixant.
} 
médicales à proximité des sites habités ou sur les aires de parcours pastorales. Ceci aura deux conséquences spatiales majeures: d'une part, les oasis perdent progressivement leur rôle de relais, la «vocation agricole » est insuffisante à assurer un niveau de vie convenable aux oasiens et l'émigration prend de l'ampleur; d'autre part, la sédentarisation qui doit être interprétée comme le signe de la crise de la fonction de circulation concerne de plus en plus de nomades. Elle s'effectue autour des oasis avec lesquelles ils étaient liés ou à proximité de lieux qui n'étaient jusque-là habités que temporairement alors que les parcours pastoraux subissent une désaffection.

En Libye, la coupure entre l'intérieur et le littoral a été autant marquée, sans doute, par la résistance à l'avancée coloniale italienne que par sa brièveté. Ici, point de traces de l'esquisse de politiques de développement dans lesquelles s'inscriront par la suite les Etats nationaux, celles que l'on retrouve dans la littérature coloniale en France sous le nom «d'œuvre française » pour reprendre le titre de la dernière partie de l'ouvrage de Robert Capot-Rey sur le Sahara français (1953). En ce sens, il est probable que la période coloniale -au sens large- a eu plus d'effets déstructurant et moins de tentatives de substitution d'un nouveau modèlent en Libye que dans l'aire française voisine.

L'urbanisation intégration ${ }^{7}$ correspond à la période où l'interventionnisme des Etats nés des indépendances se concrétise par une vigoureuse politique d'intégration nationale et par conséquent de consolidation du territoire hérité de la colonisation. Ce mouvement part des villes vers les campagnes et du littoral vers l'intérieur et c'est donc avec un certain décalage chronologique que le Sahara est touché par le mouvement. Celui-ci prend deux formes, la croissance rapide des agglomérations par croît naturel et migratoire (la sédentarisation et l'arrivée de fonctionnaires) et l'intégration par la création ou

\footnotetext{
${ }^{7}$ L'expression est d'Emmanuel Ma Mung, «l'urbanisation-intégration : la ville au service de l'Etat (Sbiba, Tunisie centrale) », in P. Signoles (dir.), Petites villes et villes moyennes dans le Monde arabe, Fascicule de recherche $n^{\circ} 16-17$, Tours, URBAMA, 1986.
} 
l'amélioration d'axes de circulation qui relient les villages entre eux et au nord.

Cette période est pleinement celle de « l'urbanisation des campagnes » avec l'émergence d'une strate de centres ruraux et de petites villes aux caractères homogènes, dotés de fonctions de base dans les domaines de l'éducation, de la santé et de l'administration. Les populations s'installent du centre vers la périphérie selon l'ancienneté de l'arrivée dans les villages oasiens ou bien par quartiers situés dans l'axe de l'aire de parcours pastoral dans le cas des villes-nomades. Mais dans les deux cas, la segmentation est partout très poussée.

Une rupture durable se dessine entre Nord et Sud du Sahara maghrébin car tandis que les agglomérations de la partie septentrionale se rapprochent dans leur évolution de celles du reste des territoires nationaux, dans la partie méridionale, comme l'illustre le cas du Fezzan, l'intervention des autorités publiques est beaucoup plus marquée et contribue à la multiplication des «villes d'Etat », Laayoune au Maroc, Tamanghasset en Algérie, Sebha et Koufra en Libye.

L'urbanité est un mouvement récent qui concerne surtout les agglomérations où le mouvement d'urbanisation se ralentit et où, la sédentarisation n'a plus la même ampleur qu'auparavant. Le passage ou non à cette phase témoigne d'un degré d'intégration inégal au système spatial de l'urbanisation-intégration. Cette inégalité est source de différenciation spatiale non seulement entre les agglomérations d'une même strate mais aussi entre les régions sahariennes, tant varient les politiques -et leur efficacité- selon les Etats et entre les régions de chacun d'eux.

Les itinéraires pastoraux disparaissent alors que se renforcent le réseau régional et l'intégration au réseau national. Pour autant, la différenciation nord-sud du rapport entre les dynamiques urbaines et celles des réseaux s'accroît. Les agglomérations des marges septentrionales sont de plus en plus intégrées aux régions littorales mais de moins en moins avec le Sahara central. Les dynamiques intra-urbaines se caractérisent par le ralentissement des rythmes de croissance démographique des années 1970 et 1980 et une atténuation des 
segmentations, ces divisions sociales de l'espace urbain fondées sur le regroupement des habitants par appartenance tribale ou origine géographique. Cette tendance a commencé dans le Sahara central, où l'exode rural était achevé depuis dix à vingt ans selon les lieux mais où les flux de réfugiés et de migrants contribuent à un nouveau processus de division, fondé notamment sur la nationalité, provoquant une fragmentation urbaine des agglomérations.

La présence, ou non, de villes au Sahara est donc étroitement corrélée à celle des réseaux, commerçants, nationaux et aujourd'hui transsahariens dans le Fezzan et tout le Sahara central.

\section{LECTURES ET RELECTURES GÉOGRAPHIQUES DU SAHARA}

\section{UNE GÉOGRAPHIE SAHARIENNE RURALE ET FRACTIONNÉE PAR LES AIRES CULTURELLES}

La géographie saharienne est l'héritière de la tradition de récits de voyages écrits par les explorateurs qui empruntent les pistes sahariennes, parfois à leurs risques et périls, durant les XVIII ${ }^{\text {ème }}$ et XIX ${ }^{\text {ème }}$ siècles, souvent pour le compte des sociétés de géographie européennes, friandes d'informations sur des espaces méconnus et donc fascinants, voire mythiques. La domination coloniale du Sahara, à la charnière des XIX ${ }^{\mathrm{ème}}$ et $\mathrm{XX}^{\text {ème }}$ siècles, entraîne un glissement vers une dimension scientifique des travaux, laquelle se renforce jusqu'aux décolonisations des années 1960, notamment, pour le domaine français, sous l'égide de l'Institut de Recherches Sahariennes (IRS) d'Alger avec entre autres les deux grands géographes que sont Robert Capot-Rey et Jean Despois. Cette géographie, on peut encore l'apprécier à travers la lecture des Bulletins de Liaison Saharienne ou les Travaux de l'IRS. Sans revenir sur l'ensemble des thématiques, retenons quelques points particulièrement intéressants car ils ont contribué, parfois à l'insu de leurs auteurs, à la diffusion de poncifs durablement en usage dans la lecture des sociétés sahariennes.

La géographie saharienne est placée sous le double signe de la découverte des espaces et des populations et de l'accumulation de connaissances. En marge de brillants travaux menés par des scientifiques 
qui ont sillonné le «Sahara français », et des militaires, à la fois observateurs, fins connaisseurs et acteurs chargés du maintien de l'ordre et de l'encadrement administratif, une fabrique de "petite géographie» (D. Retaillé, 1989) s'esquisse. Peu à peu, la collecte riche et diversifiée d'informations sur les tribus, leur composition, les plans de palmeraies ou de villages, les genres de vie nomades et sédentaires participent de la construction de catégories qui figent le Sahara dans des images promptes à satisfaire les imaginaires en quête d'exotisme.

Les ensembles oasiens, véritables foyers de peuplement du Sahara, constituent le socle des travaux de géographie régionale. De nombreuses monographies sont lancées sur les différentes régions qui composent le Sahara avec pour objectif d'effectuer une description exhaustive, physique ou humaine - sommes parfois inégalées depuis - portant sur des espaces peu étudiés par la suite, tels le Fezzan par Jean Despois (1946), le Souf par Claude Bataillon (1955), le Gourara par Jean Bisson (1957). Ces travaux sont intitulés selon le nom de ces ensembles régionaux oasiens circonscrits par l'alternance des «pleins » et des « vides » que tracent sur les cartes les inégales densités de population. La démarche empruntée par les chercheurs est logique : en l'absence d'informations, la priorité est au comblement des lacunes et à l'accumulation des connaissances sur l'ensemble du Sahara mais elle ne sera pas toujours comprise car progressivement une vision du désert se construit, celle d'oasis considérées comme des isolats spatiaux, sortes de cellules autarciques. Or rien n'est plus faux que cette image qui renvoie à un contexte datable, celui de la crise que traverse le Sahara après le déclin du commerce transsaharien et qui coupe les oasis des réseaux de circulation le long desquels elles assuraient une fonction de relais.

Le classement du Sahara dans le champ de l'urbain ou du rural est une question récurrente du discours géographique. Pour Robert Capot-Rey (1953), «on peut hésiter à reconnaître des villes au Sahara tant les caractères ruraux et urbains sont intimement mêlés » dont les principales distinctions apparaissent à travers l'habitat, mieux entretenu dans les villes, et les fonctions, telles que l'artisanat, le commerce de négoce et la taille des souks. Cette difficulté à cerner la ville renvoie au Sahara sous domination coloniale, à un espace qui a « perdu » ses villes comme Mourzouq, brillant exemple de la civilisation citadine du Sahara jusqu'au XIX ${ }^{\text {ème }}$ siècle, encore qualifiée de ville dans les années 1950 alors que, marginalisée et délabrée, elle ne compte plus que quelques centaines d'habitants. Cette différenciation formelle entre mondes rural et urbain est reprise quarante ans plus tard par exemple par Abdelfettah Kassah (1993) à propos de l'oasis de Tozeur où le caractère rural ou urbain est défini dans l'habitat, maison traditionnelle versus villa, l'aspect des rues, goudronnées ou pas, 
ou la présence ou non d'animaux. Pourtant les débats actuels ne visent plus à dissocier ce qui est urbain de ce qui ne l'est pas mais cherchent plutôt à réfléchir à ce qui fait la ville dans le Sahara contemporain.

Il existe en effet un débat scientifique remettant en cause les poncifs qui ont sous-tendu les représentations du Sahara. L'association trop rapide entre Sahara et ruralité ne paraît plus en mesure d'aider à cerner avec pertinence les dynamiques spatiales qui transforment aujourd'hui les foyers de peuplement du désert. L'analogie entre vide démographique et espace délaissé fait partie de ces faux-semblants qui conduisent à négliger la dimension « sensible » des espaces qui, à défaut d'être habités, n'en ont pas moins une place déterminante dans la conception territoriale des populations sahariennes. Enfin, la « totalité spatiale » que représenteraient le ksar et la palmeraie semble contredite par la vivacité des réseaux de circulation sahariens. Toutefois, ces débats ne sont pas abordés de la même manière sur les versants maghrébin et sahélien du Sahara.

Les géographes du Sahel opèrent, pour répondre à leurs questionnements, une «montée vers le Nord ». Leurs travaux sont inscrits dans des problématiques en prise avec les spécificités de leurs terrains et portent sur les échanges informels ou les circulations (E. Grégoire), les représentations ou les conceptions du territoire (D. Retaillé), Or ces terrains sont de plus en plus éclatés ou du moins ne sont plus considérés sous l'angle d'ensembles régionaux clairement délimités. Les géographes, comme tous les chercheurs en sciences sociales de cette aire géographique, prennent acte de la porosité des frontières dans les pratiques et non pas théoriquement, en partant de la vision géopolitique formalisée lors du Congrès de Berlin ou du principe d'intangibilité des frontières fixé après les indépendances.

La géographie du Sahara maghrébin a privilégié une lecture du Sahara selon le « gradient nord-sud » car ce versant du désert n'était considéré que comme le prolongement, vaste et périphérique, du littoral. Dans cette optique, le Sahara maghrébin est d'abord pensé à travers le rapport des Etats à cet espace et aux sociétés qui y vivent. Durant les années qui suivent les indépendances, la question essentielle est celle de l'intégration du Sahara aux territoires nationaux, objectif fondamental pour les autorités nationales, soucieuses de légitimer les frontières internationales et de construire des territoires étatiques "cohérents », en rupture avec les mécanismes de la dépendance qu'avaient mis en place les colonisateurs. On porte alors attention aux découpages administratifs, aux aménagements, à l'urbanisation, à la croissance démographique, bref à tous les éléments qui témoignent d'une plus ou moins grande intervention 
étatique dans le développement des portions nationales du Sahara. Par la suite, des études fines permettent de pointer l'émergence d'acteurs qui initient de nouvelles dynamiques locales, liées à une importante émigration, à l'agriculture privée, en expansion rapide et à la consolidation de la trame urbaine. En revanche, les travaux de recherche publiés décroissent en nombre du nord vers le sud. Ces disparités dans la couverture géographique du Sahara limitent notre compréhension des dynamiques socio-spatiales qui le traverse puisque nous connaissons mieux ses marges que son centre. Le déficit en travaux sur les espaces méridionaux du Sahara maghrébin n'a pour ainsi dire pas permis d'en repérer les mutations les plus récentes.

La lecture géographique du Sahara selon le gradient nord-sud pose aujourd'hui de plus en plus problème car c'est sur les marges, comme souvent dans les modèles d'interprétation, que les limites de cette vision se manifestent le plus nettement. La déconstruction des grilles de lecture héritées de la période coloniale est encore fortement ancrée dans le découpage politique en huit Etats (Maroc, Algérie, Tunisie et Libye au Nord, Mauritanie, Mali, Niger et Tchad au Sud) et deux aires culturelles (le Monde arabe et l'Afrique noire) du Maghreb et du Sahel. Ces divisions ne sont évidemment pas sans fondements car elles renvoient à des processus différents de construction territoriale sous impulsion coloniale puis nationale. Elles s'expliquent aussi par l'inégale propension des Etats maghrébins et sahéliens à maîtriser et à intégrer le Sahara aux territoires nationaux. Les premiers ont mené le mouvement d'intégration avec force alors qu'il reste encore en projet au Sud. L'inégalité en ressources financières est un facteur essentiel d'explication puisque les Etats sahéliens n'ont jamais réellement eu les moyens d'une politique pour désenclaver ou développer les régions sahariennes sur lesquelles ils exercent leur souveraineté. Mais inégalité en moyens financiers n'explique pourtant pas tout et notamment le fait que le Sahara central ait été une zone de conflits, secouée par les guerres entre le Tchad et la Libye et au Sahara occidental ou les conflits internes, principalement entre les Touaregs du Mali et du Niger et les Etats centraux.

Le Sahara n'était évidemment pas divisé dans les approches de manière artificielle, seulement ces divisions perdent aujourd'hui en pertinence. Du centre du Sahara vers ses «périphéries » littorales et sahéliennes, part un gradient inverse, fondé sur les dynamiques impulsées par la circulation des migrants et des marchandises dont l'importance est telle qu'il contribue à redessiner la géographie de nombreuses villes et régions. 


\section{UNE ENTRÉE URBAINE SUR LE FEZZAN CONTEMPORAIN}

Le Sahara est d'abord urbain, mais il l'est autrement. Aborder le Sahara au prisme de l'urbain devient une nécessité largement soulignée par les travaux menés depuis deux décennies ${ }^{8}$. Dans cette perspective, la notion même d'urbain n'est pas satisfaisante et il convient donc de la préciser et de tenter de définir quelques-uns de ces aspects.

L'urbanisation n'en est qu'une facette. Il est légitime qu'elle ait focalisé l'attention des chercheurs tant elle fut spectaculaire dans tout le Sahara. Mais en inscrivant les études urbaines dans la continuité du processus d'urbanisation, ne risque-t-on pas de poser des questions pertinentes à une échelle d'analyse mais pas aux autres ou de privilégier des approches justifiées ailleurs mais pas ici ? Odette Louiset (2000) souligne que «les phénomènes et processus considérés comme majeurs dans l'histoire et l'organisation de nos villes (sous-entendu européennes)... ne peuvent être abordés comme des évidences» et rappelle que «ce que nous appelons ruralité dans les villes africaines, marginalité dans les slums..., c'est de la ville pour ceux qui ne sont pas moins urbains, mais qui le sont autrement ». Cette optique incite désormais à relativiser l'intérêt porté au processus d'urbanisation afin de repérer comment se manifeste l'urbain dans le Fezzan aujourd'hui. Certaines manifestations y relèvent de la plus simple matérialité, fondée sur les décalages, les dysfonctionnements, les hésitations qui témoignent de la construction pas à pas d'un espace vécu alors que d'autres relèvent de l'idéel. Trois pistes méritent une attention particulière selon cette dernière optique : l'urbain est projet et transaction qui lie ceux qui détiennent le pouvoir à ceux qu'ils gouvernent ; revendication lorsqu'il transparaît dans le discours comme un symbole du confort et de la norme sociale ; pratique lorsque l'on évoque les regroupements communautaires et les nouvelles formes de sociabilités.

L'enjeu émergent est celui d'une conceptualisation de l'objet ville, enjeu qui, séduisant du point de vue théorique, s'avère évidemment délicat dans son application. A ce prix, peut se trouver une sortie permettant de ne plus considérer la ville seulement comme le produit de l'aménagement, de l'échange ou le réceptacle de diverses migrations mais aussi comme un objet qui produit de la société - une configuration sociétale dirait Jacques Lévy $(1994,1999)$. Cette question constitue une passerelle avec le sujet de cette recherche à condition de l'aborder selon les spécificités de l'aire

${ }^{8}$ Notamment dans les articles consacrés au Sahara dans les Fascicules de recherches URBAMA, cités en bibliographie (J. Bisson, 1989, 1991, 1993 et J. Fontaine, 1996). 
saharienne où le bouleversement social a largement accompagné le bouleversement spatial. La rapidité de ces dynamiques, et le fait qu'elles se poursuivent actuellement, contribuent à multiplier les questions. Mais il est à craindre que les réponses manquent encore, pour les scientifiques, certes, et surtout pour les habitants et les acteurs locaux, notamment ceux du Fezzan, cette vaste région du Sahara libyen, sur laquelle porte ce travail.

La première partie tourne autour d'une question centrale : qu'est-ce qui institue la ville? L'approche diachronique, entendue ici comme « histoire regardée avec les yeux de la géographie, c'est-à-dire en privilégiant la lecture de la dimension spatiale pour approcher les causes et les effets de ces changements » (J. Lévy, 1997), permet de réfléchir à l'évolution de l'organisation spatiale afin de comprendre la place qu'y occupe l'urbain. Il apparaît qu'à chaque période le ou les acteurs dominants, ceux qui façonnent l'espace et contribuent à son organisation, donnent un sens et un contenu différents de la notion d'urbain. Au commerce transsaharien correspond la ville, lieu de rencontres, d'échanges, de pouvoir. Durant la colonisation, les autorités militaires réduisent ces fonctions et déplacent les sites, privilégiant le contrôle des circulations pastorales et commerçantes plutôt que celui des foyers de peuplement oasiens. Par la suite, l'Etat, tout en confortant la trame coloniale, multiplie les agglomérations afin de contrôler mais surtout de permettre à l'ensemble des populations d'accéder à des normes de confort similaires au reste du pays. Actuellement, ce modèle est remis en cause par la réactivation des circulations transsahariennes entre la Libye et l'Afrique qui, sans constituer une véritable rupture, n'en conduit pas moins à nuancer le principe de relations exclusives entre échelons nationaux et locaux.

La seconde partie présente les principales traductions spatiales de ces mutations, tant urbaines que rurales. Sur bien des points, la coupure apparaît synthétique entre ces deux objets et si elle l'est, c'est sans doute parce que les acteurs n'agissent pas dans un champ cloisonné mais qu'au contraire ils saisissent les opportunités et les failles perceptibles dans un système national qui se rétracte. On rejoint sur ce point des constats établis dans l'ensemble du Monde arabe et on repense en conséquence à des concepts utilisés à ce propos. La fragmentation urbaine signifie-t-elle la crise de la ville ? N'y a-t-il de campagnes que sous l'influence des villes? Le local, représenté par ses acteurs, est-il un contrepoids au national ? L'analyse spatiale nous donne des pistes sur ces questions, et sur d'autres, mais aussi sur les intentions d'acteurs divers et aux marges de manœuvre bien inégales. 
Finalement se pose le problème du sens de l'urbain, thème central de la troisième partie. Par-delà les chiffres et les formes matérielles qui expriment le processus d'urbanisation, comment les populations expriment-elles leurs urbanités? Chercher «l'esprit du lieu» comme Jacques Lévy (1999) en géographie ou Jean-Claude Guillebaud (2000) en littérature le suggèrent ne va pas de soi et risque même dans les villes du Sahara libyen de mener au «non-lieu » (M. Augé, 1992), «sans identité, sans épaisseur historique, incapable de provoquer ces relations interindividuelles qu'encourage normalement un véritable espace social » (G. di Méo, 1995). Le questionnement peut paraître de prime abord artificiel mais souvent encore la référence au lieu reste attachée dans nos représentations à celle du patrimoine, conduisant à regretter que « de plus en plus, la ville s'efface au bénéfice de l'urbain » et à ne voir comme futur que « la juxtaposition de morceaux de villes qui ne se fondent pas dans un tout » (R. Escallier, 1998).

Mais parle-t-on de la mort de la ville ou de celle d'une conception de la ville? L'urbain a parfois des connotations d'uniformité, de quantité, d'anonymat en opposition à la ville, elle clairement circonscrite, porteuse d'une identité partagée par ses habitants. Or une telle ville est peut-être plus marginale qu'il n'y paraît au Sahara. Avant l'urbain pensé dans un cadre national, il y avait d'autres conceptions du territoire, pas forcément antithétiques de la ville, comme l'a suggéré Denis Retaillé (1989). C'est peut-être plus à cet itinéraire qu'il convient de réfléchir, en considérant alors la ville comme projet plutôt que comme un idéal perdu, mais certainement sous d'autres formes spatiales.

Il ne s'agit pourtant pas de faire l'économie d'une description des formes et de leur évolution car si l'urbain peut se définir autrement que par la forme, c'est dans un espace donné que les sociétés sahariennes déploient leur quotidien et sur cet espace nous ne disposons que d'informations lacunaires et éparses. Entendons-nous bien, nous souscrivons au constat d'Odette Louiset pour qui « la morphologie ne suffit pas à rendre compte et surtout à comprendre la ville comme lieu et comme concept » mais cette position pose cependant à notre sens un autre problème : travailler sur le Sahara libyen, c'est travailler sur les marges d'un Etat marginal au plan des travaux scientifiques et si passer outre une lecture strictement spatiale de la ville est nécessaire, dans quelle mesure doit-on tenir compte du cadre spatial ? On conviendra qu'il n'est pas neutre et cette absence de neutralité rend, à notre sens, nécessaire l'appréhension précise du cadre. Or rien ne serait plus faux, afin d'esquiver cet impératif, que de l'assimiler à un cadre maghrébin type. Considérant que si sans société, il n'y a pas d'espace mais qu'en retour, sans éclairage spatial, la compréhension d'une société est 
incomplète, je prends le parti, parfois, de privilégier une démarche centrée sur le décryptage des faits spatiaux plutôt que sur la lecture sociale de ces faits.

Les apparences plaident pour l'absence de lieux et donc de possibilités d'interactions sociales. Or les lieux dans le Sahara urbain émergent, au même titre que la ville d'ailleurs. Ils ne sont jamais une évidence immédiatement perceptible dans le paysage de l'agglomération tant la privatisation de l'espace est partout flagrante au détriment des lieux publics. Sommes-nous alors dans un de ces lieux où les obstacles seraient tels que l'urbanité et la ville comme lieu de production de société sont impossibles? L'approche scientifique exclut des réponses catégoriques et des questions qui ne le sont pas moins mais plaide plutôt pour la durée, un temps long de l'émergence de la ville. 


\section{Première PARTIE}

\section{LES VILLES DU FEZZAN ENTRE TERRITOIRE D’ETAT ET CARREFOUR}

«Le territoire des Kel Ahaggar (est) aujourd'hui disparu... alors que la société qui a construit le dispositif a vécu ». Ce paradoxe relevé par Denis Retaillé (1998) à propos des Touaregs du Hoggar vaut pour d'autres espaces sahariens et conduit à questionner la dimension temporelle du processus d'urbanisation. Si une société saharienne, telle celle des Kel Ahaggar, a donné sens et s'est appropriée un espace en le territorialisant, et bien de même, l'intégration de cet espace à d'autres, beaucoup plus étendus, entraîne un nouveau processus de territorialisation à l'initiative de nouveaux acteurs. Dès lors, peut-on considérer la généralisation de l'urbain comme le reflet de nouvelles territorialités ? On devine la réponse positive mais il importe de définir selon quelles modalités ce processus se met en place. Dans le Fezzan, une constante réside dans la trame du peuplement : on ne compte pas aujourd'hui beaucoup plus de sites habités qu'au début du siècle. Mais la hiérarchisation qui s'opère actuellement pose question. Pourquoi les carrefours se succèdent-ils? Pourquoi ne trouve-t-on pas ici cette dynamique cumulative qui aurait fait des centres historiques de l'échange, encore actifs il y a quelques décennies, les centres administratifs d'aujourd'hui ?

L'opposition dialectique, et didactique, entre l'urbanisation du haut en bas et l'urbanisation remontante proposée par Gilles Sautter (1982) permet d'esquisser un cadre explicatif. Certes, ces deux mouvements sont interdépendants et varient en intensité selon les lieux et les périodes. Mais une lecture globale des dynamiques socio-spatiales du Fezzan rappelle avec force que les cycles urbains qui s'y succèdent doivent à un moment donné à un acteur dominant. Il apparaît, pour aller vite, que le commerce transsaharien crée la ville, la colonisation la garnison et l'Etat l'agglomération. En disant les choses de manière si lapidaire, nous prenons le parti de réduire le champ de la perspective diachronique ébauchée mais aussi de préciser ce que nous entendons par organisation spatiale. Le terme se justifie dans les cadres commerçant et national mais il perd de sa pertinence à propos de la colonisation, brève et partielle, essentiellement déstructurante. Quant à la période contemporaine, plus qu'une rupture avec 
celle de l'intégration nationale, elle laisse apparaître les limites du rôle de l'Etat tout en s'inscrivant dans le cadre qu'il a instauré. 


\section{CHAPITRE 1 - LE FEZZAN, UN CARREFOUR SAHARIEN}

Le Fezzan est avant tout un carrefour saharien, inscrit dans la durée, sur les grands axes du commerce caravanier. Il présente les éléments caractéristiques de toutes les régions similaires, une localisation intermédiaire entre les deux « littoraux », méditerranéen et sahélien, une trame de peuplement dense et stable, une organisation fondée sur le rôle de relais commerçant. Avant d'évoquer ces trois points, nous reviendrons sur un principe qui nous semble essentiel, celui des échelles de «lecture » des espaces oasiens.

Pourquoi trouve-t-on des sites oasiens dans le Sahara ? A l'échelle locale, un lieu habité dépend de la proximité de l'eau. Pourtant, il est nécessaire de nuancer le rôle de l'eau car «ce ne sont pas tant les points d'eau, rares à l'état naturel, qui ont attiré les établissements humains. Il est beaucoup plus vrai de dire que puits et sources, comme les cultures qui en dépendent, ont été créés à proximité des villages » (J. Despois, 1946). Rester à l'échelle locale, c'est donc conforter une opinion erronée, celle qui tend à enfermer les villages dans un rôle uniquement productif, au centre d'un terroir oasien.

« Pour une ville du désert, il n'y a pas de mort violente, rien qu'une agonie prolongée » soulignait Robert Capot-Rey (1953). Il faut entendre par-là que la disparition d'un site habité ne s'effectue qu'avec sa marginalisation progressive vis-à-vis d'un système spatial plus vaste. A l'échelle du désert, la présence des villes s'explique par leur rôle de relais sur un axe de circulation. L'intensité de la fonction de relais varie selon l'importance du centre dans le réseau commerçant. En conséquence, une ville qui périclite a eu un rôle dans un système de circulation qui disparaît mais elle n'en a plus dans ceux qui se reforment ailleurs. Ce principe permet d'analyser l'évolution des villes et de leur situation dans le territoire fezzanais sur le long terme.

Il est donc utile de raisonner à deux échelles pour comprendre les mutations. L'échelle locale correspond au lieu, en l'occurrence l'oasis, elle permet de décrire un paysage et les pratiques de ceux qui y vivent alors qu'à l'échelle globale, on peut comprendre pourquoi et comment le lieu est lié à d'autres lieux. Ces deux approches permettent de donner sens à la question de la localisation mais aussi à la fonction de circulation qu'assument les nomades. Ce double jeu d'échelles invite aussi à souligner un point fondamental : si l'on a longtemps vanté l'intensivité du système agricole oasien afin de légitimer une vision « agraire » qui l'apparenterait 
aux campagnes que l'on trouve sous d'autres latitudes, on en a oublié sa fonction essentielle, celle de la circulation. L'espace rural peut en fin de compte se réduire à sa fonction de point de ravitaillement et d'échange à la croisée des routes transsahariennes.

\section{LES ÉCHELLE DU CARREFOUR FEZZANAIS}

Théodore Monod (1968) a proposé une lecture devenue classique de la structuration spatiale du Sahara en «compartiments méridiens» (fig.2). Ces «compartiments», ou « fuseaux », sont qualifiés de «positifs» ou "négatifs ». Les fuseaux négatifs correspondent à des régions d'ergs (massif de dune) de regs (plaine de sable ou de petits cailloux) ou de hamadas (surface formée par l'affleurement d'une couche dure) pour l'essentiel vides de population à l'exception de quelques noyaux oasiens tels le Kawar, à l'est du Ténéré (IV) ou Koufra dans le désert Libyque (VI), dont la fonction était celle du ravitaillement ou du négoce. Ces trois «vides» séparent quatre fuseaux positifs, Maure (I), Touareg (III), Toubou (V) et Vallée du Nil (VII), laquelle déroge aux principes d'organisation évoqués ici. Dans les trois premiers cas, le fuseau présente des caractères identiques, à fondement nomade et ethnique.

L'étonnante longévité du Fezzan comme carrefour s'explique par une localisation exceptionnelle. Vers le nord, d'abord, la Tripolitaine est nettement plus méridionale que les autres capitales du Maghreb, ce qui réduisait considérablement les trajets terrestres. Vers le sud, le Fezzan est la seule région à cette latitude à se trouver à l'intersection de deux fuseaux, Touareg à l'ouest, Toubou à l'est et d'axes commerciaux vers plusieurs directions majeures. Ainsi, alors que le déclin des itinéraires occidentaux, vers le Maroc et l'Algérie septentrionale, est imputé, durant l'époque moderne, à l'ouverture de nouvelles routes maritimes entre le Maghreb, l'Europe et les comptoirs de l'Ouest africain, l'itinéraire fezzanais reste plus rentable pour l'échange des objets précieux et la traite des esclaves. 
Figure 2 : La structuration spatiale du Sahara

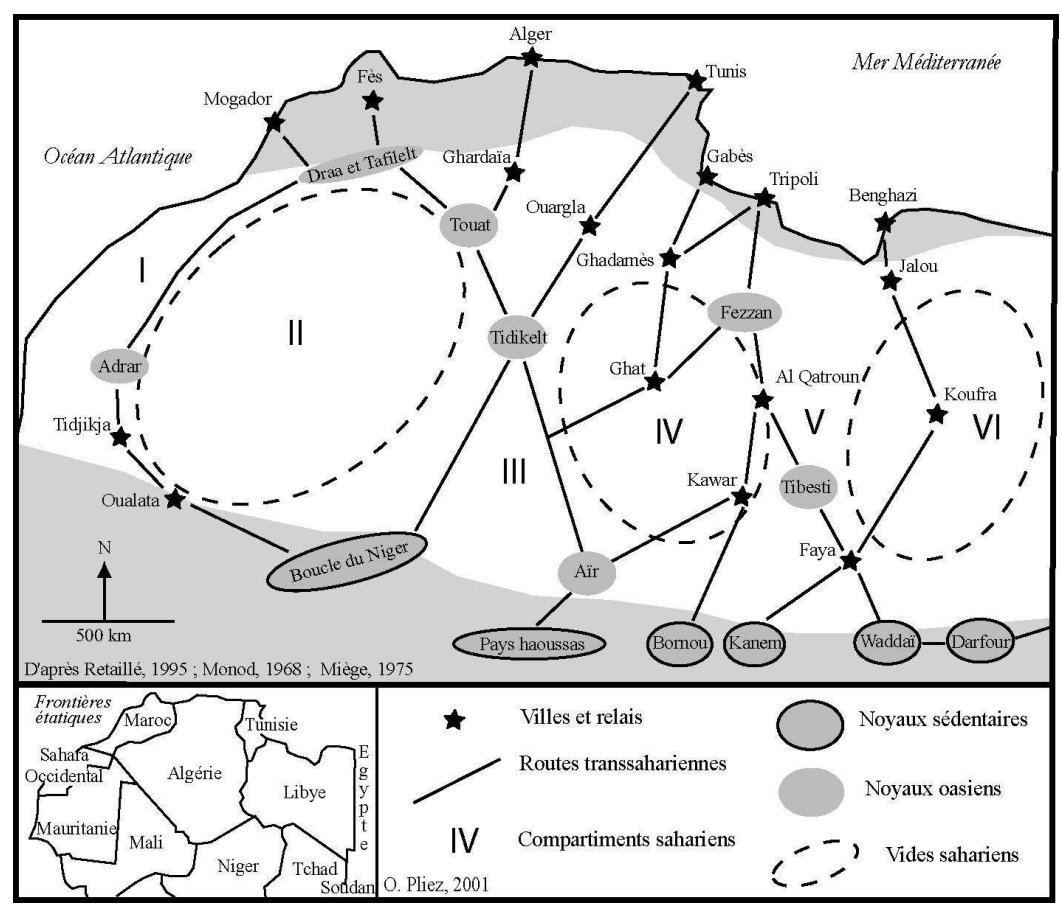

Les limites du Fezzan varient dans le temps et dans l'espace. On s'accorde néanmoins à circonscrire son noyau central autour de trois axes longitudinaux, correspondant plus ou moins à des vallées d'oued (rivières). Ils se caractérisent par un peuplement continu sur une centaine de kilomètres de long, séparés les uns des autres de 50 à 100 kilomètres (fig.3).

Au centre, se situe le Wadi Ajal (la vallée de la mort), baptisé Wadi Al Haya (vallée de la vie), après la rénovation rurale effectuée dans le cadre de projets de développement durant les années 1970 et 1980 . C'est là que le peuplement est le plus continu, même si l'on distingue deux parties, le Wadi Gharbi, occidental, densément peuplé, d'Oubari à Fjej et le Wadi Sharqi, oriental, d'Al Abiod à Al Graya. Ses terres, dépôts limoneux étroitement circonscrits par l'Erg d'Oubari au nord et la hamada (plateau) de Mourzouq au sud sont les plus fertiles du Fezzan. C'est aussi là que le khamessa (métayage) a toujours été le plus important car la richesse des terres a incité les familles les plus riches des autres vallées à y acquérir des terres (J. Lethielleux, 1948). 
Figure 3 : Le Fezzan

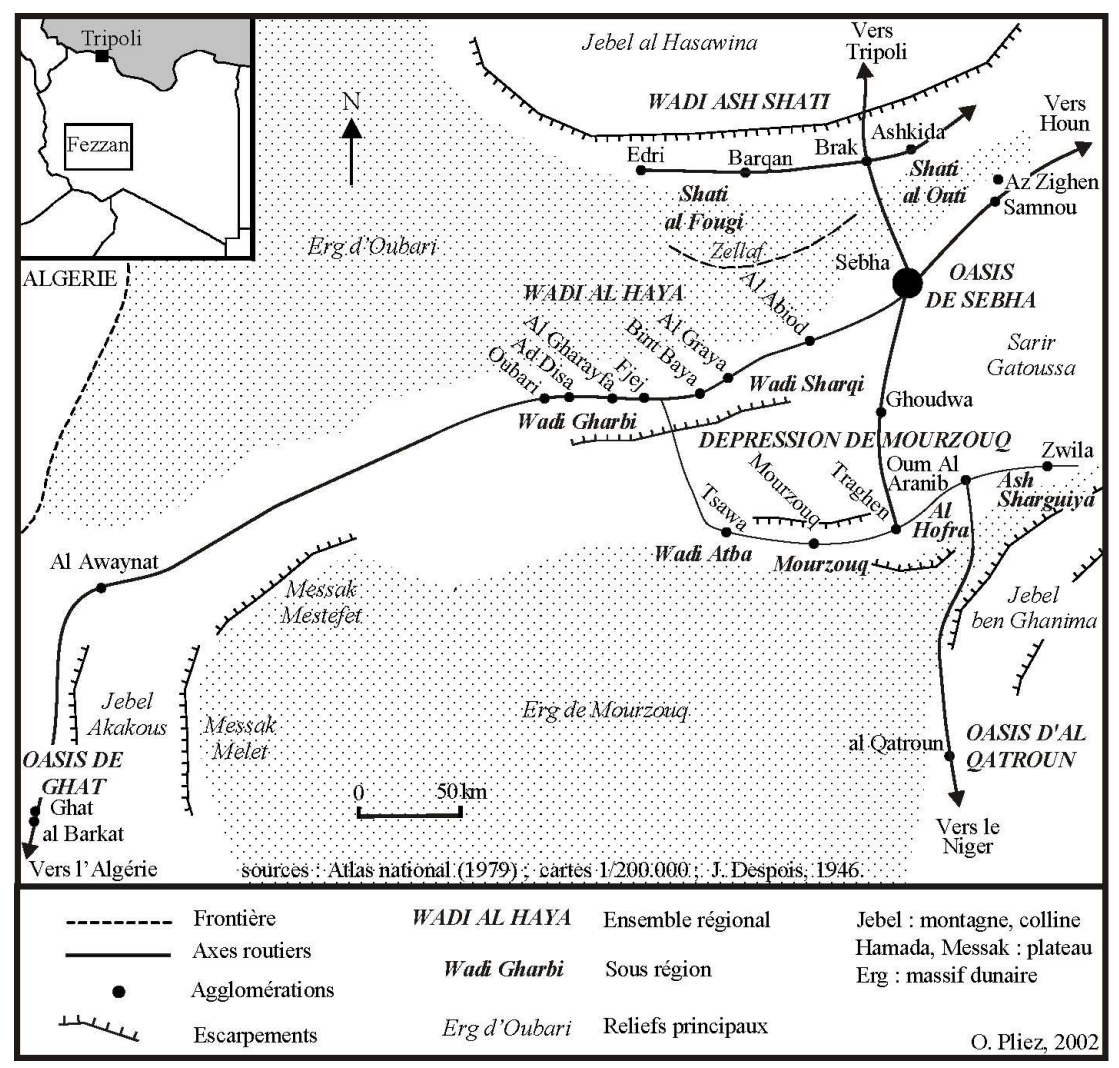

Au Nord, le Wadi Ash Shati s'organise sous la forme de groupements villageois discontinus, d'Edri à Ashkida qui correspondent à la localisation des terres cultivables, plus rares que dans le Wadi Al Haya. Le commerce y a toujours eu une place plus notable, en relation avec le Jebel Nefousa au Nord et le littoral syrtique (A. Cauneille, 1960). Au sud, la dépression de Mourzouq se compose aussi de noyaux de peuplement discontinus, autour de centres ruraux notables. Sur de nombreux points, elle s'apparente au Wadi Ash Shati avec peu de terres cultivables mais une accumulation du capital durablement liée au commerce transsaharien, cette fois-ci en lien avec le Sud. Enfin, deux autres aires de peuplement, moins peuplées et traditionnellement sans centres notables, finissent de composer le Fezzan, Sebha et Al Bouanis, à égale distance des trois vallées et la région d'Al Qatroun sur la route du Sahel. 
Afin d'achever cette présentation du Fezzan, il importe de revenir sur le clivage séparant les nomades des sédentaires. La vision coloniale a durablement forgé une distinction des populations sahariennes selon une gradation fondée sur la distance et la durée de leurs déplacements, du nomade, le plus mobile, au sédentaire, «l'immobile». Cette catégorisation, lorsqu'on la quantifie, restitue une image paradoxale puisque le Fezzan, unique région exclusivement saharienne de Libye, est celle qui compte en 1954 la plus faible proportion de nomades, $3 \%$, et la plus forte proportion de sédentaires, $91 \% \%^{9}$. On peut étayer ces chiffres à l'aide de deux arguments, l'un pragmatique, le Fezzan se situe dans le Sahara central, une région où l'hyper-aridité réduit les aires de pâturages et par conséquent l'activité pastorale; d'autre part, à l'intersection des fuseaux Touareg et Toubou, le Fezzan est d'abord un noyau de peuplement rural. Le second argument est idéel, à savoir que si le Fezzan est essentiellement un noyau de peuplement rural, « au sein même des sociétés dites nomades, les sédentaires sont majoritaires»(D. Retaillé, 1998). Quoique évocatrice, la spécificité fezzanaise d'une prépondérance sédentaire repose donc d'abord sur la persistance de catégories désuètes de lecture des sociétés sahariennes. A nouveau doit revenir à l'esprit l'importance du jeu des échelles : au plan local, les chiffres restituent une prépondérance numérique des populations sédentaires mais c'est à plus petite échelle que doit être pensé le carrefour fezzanais.

La situation et la fonction du Fezzan se traduisent en effet par un maillage dense relais sur les différents axes commerçants mais aussi de capitales, véritables carrefours et centres de négoce des marchandises et des esclaves qui y transitent. Depuis la période médiévale, trois capitales se succèdent, toutes situées dans la dépression de Mourzouq, Zwila à l'est, Traghen au centre, Mourzouq à l'ouest. Ce réseau est complété par plusieurs relais dans chacune des trois vallées, généralement traversées par une ou deux pistes en direction du nord dans le Wadi Ash Shati, du sud dans la Dépression de Mourzouq, du sud-ouest dans le Wadi Ajal. Cette organisation, dépeinte sommairement ${ }^{10}$, décline progressivement à partir du XIX ${ }^{\text {ème }}$ siècle alors que les puissances coloniales intensifient leur course à la conquête de nouveaux territoires.

\footnotetext{
${ }^{9}$ Selon le recensement de la population de 1954 on compte en Tripolitaine et en Cyrénaïque respectivement 7 et $12 \%$ de nomades ; 13 et $33 \%$ de semi-nomades ; 80 et $55 \%$ de sédentaires.

${ }^{10}$ Afin d'en rester à une présentation générale du cadre et donc de ne pas développer ce qui a pu être dit de manière plus détaillée par ailleurs. Nous renvoyons notamment aux ouvrages de J. Despois (1946), J. Lethielleux (1948), J. Thiry (1995), J. Bisson et J. Fontaine (1999).
} 
HINTERLAND OU PÉRIPHÉRIE ? LES ENJEUX COLONIAUX (1850 - 1950)

Trois puissances, les Ottomans, les Italiens et les Français se trouvent en concurrence dans la course au partage de l'Afrique du Nord à partir de la seconde moitié du XIX ${ }^{\text {ème }}$ siècle. Cette concurrence s'exprime dans un premier temps dans la conquête des principales régions littorales mais elle trouve rapidement des prolongements dans leur volonté commune d'étendre leur domination aux hinterlands, ces arrières-pays que constituent les espaces et les routes du Sahara. Dans cette conquête, le Fezzan n'apparaît d'abord pas comme un objectif majeur. Mais il le devient au fur et à mesure des extensions territoriales, réelles ou projetées, des acteurs en présence qui tentent de réactiver sa fonction de carrefour stratégique entre le Sahel et la Tripolitaine, plutôt que d'en faire une colonie. Durant le siècle de turbulence -d'anarchie dans le vocabulaire colonial- qui court des années 1850 aux années 1950, le Fezzan connaît une situation paradoxale de crise économique et sociale intérieure grave, la " décadence » évoquée par Jean Despois (1946) mais d'une lutte acerbe pour le contrôle des axes au sein desquels il occupe une place centrale : axes commerçants entre le Sahel et la Tripolitaine pour les Ottomans et la confrérie de la Senoussiya, relais vers la Corne de l'Afrique pour les Italiens puis trait d'union entre l'Algérie et le Tchad pour les Français.

Jusqu'au début du XIX ${ }^{\text {ème }}$, les Karamanli, qui détiennent le pouvoir en Tripolitaine, bénéficient de la bonne conjoncture économique des échanges caravaniers qui depuis le Bornou, via le Fezzan, animent le commerce maritime du port de Tripoli. Mais la prise d'Alger par la France en 1830 et l'éradication de la piraterie en Méditerranée entraîne une crise économique et la recherche par les autorités ottomanes de nouvelles sources de revenus (P. Soave, 2001). La taxation du commerce caravanier croît jusqu'à ce que le Fezzan entre en rébellion. Les autorités de Tripoli procèdent à la reprise en main de la Tripolitaine qui devient province ottomane, puis du Fezzan d'abord en négociant avec Abd el Gelil, le chef de la puissante tribu des Ouled Sliman puis en soumettant la région en 1841 (H. Duveyrier, 1846). Le Fezzan, dont la capitale reste Mourzouq, devient alors kä̈mankalik de l'Empire ottoman, dépendant du gouvernement de la Tripolitaine. L'âpre conquête territoriale à laquelle Français et Ottomans se livrent sur les marges nord du Sahara, entre confins tripolitains et Suds algérien et tunisien, pousse ces derniers à renforcer leur contrôle indirect sur le Fezzan qui est progressivement réduit au statut d'hinterland de la Tripolitaine (A. Martel, 1991).

C'est dans ce contexte de conquête coloniale qu'à partir 1850, le Fezzan devient terre de mission pour la Senoussiya, une puissante 
confrérie saharienne (J.L. Triaud, 1995). Vingt et une zawiyas y sont créées durant le $\mathrm{XIX}^{\mathrm{e} m e}$ siècle, contre quarante-cinq en Cyrénaïque d'où rayonne la confrérie, et 18 en Tripolitaine pourtant beaucoup plus peuplée. Ces centres regroupaient une mosquée, une école, la résidence du cheikh et de sa famille, une pension pour les voyageurs, un hébergement pour les caravanes et les réfugiés (A.A. Ahmida, 1994). Les zawiyas cumulent donc deux fonctions qui sont au cœur du projet senoussi, celles de haltes sur les grandes routes commerciales transsahariennes et celles de lieux spirituels

En bâtissant des zawiyas, la Confrérie construit aussi l'amorce d'un réseau urbain dont la logique ne se comprend que par sa localisation par rapport à des aires d'influence étendues. Henri Duveyrier en témoigne à Waw où «les frères de l'ordre d'Es-Senoûsi commencent à bâtir une ville » (in J.L. Triaud, 1995) dont il ne reste plus trace aujourd'hui. Mais surtout, dès 1850, les principaux centres d'implantation de la Senoussiya sont Ghat, Ghadamès, Soukna, Zwila et Waw, suivis d'Al Qatroun lorsque le réseau senoussi s'étend vers le sud, c'est-à-dire les «portes d'entrée » balisant les grands itinéraires de circulation transsahariens à partir du Fezzan.

La seconde moitié du XIX ${ }^{\text {ème }}$ siècle est pourtant marquée par le déclin de la plupart des routes transsahariennes : vers le Maroc, le Sultan Moulay Abdurahman tente d'endiguer la pénétration européenne mais de ce fait, il limite aussi les exportations sahariennes (J.L. Miège, 1975) ; la résistance des grands chefs arabes à la poussée française vers le sud de l'Algérie, et par conséquent le Sahara, coupe les axes commerciaux du Sahel vers les ports algériens jusqu'à la chute du bey de Constantine en 1840, celle d'Abd el Kader en 1848 et la prise de Laghouat en 1852 (O. Meynier, 1930).

Le Fezzan occupe donc une place paradoxale car la crise des itinéraires sahariens occidentaux et centraux se conjuguent avec les actions menées en parallèle par la Sublime Porte et la confrérie de la Senoussiya plus à l'est. Les autorités ottomanes prennent le contrôle des deux grandes places commerçantes de Mourzouq et de Ghadamès, pendant que les autorités de la Confrérie établissent leur pouvoir sur le Waddaï et sa capitale, Abéché, activant un axe commerçant dont les ramifications courent jusqu'à Tombouctou au sud du désert et jusqu'à Tripoli et Benghazi au nord.

De la sorte, alors qu'à un siècle de distance, Henri Duveyrier en 1846 et Jean Despois en 1946 utilisent le même terme de décadence pour qualifier la région, il apparaît qu'entre ces deux dates, le Fezzan connaît un regain d'activité marchande. Les transactions commerciales atteignent des niveaux historiques à Ghat en 1875 et Tripoli capte près des $9 / 10^{\text {ème }}$ des flux de marchandises (produits et esclaves) qui traversent le Sahara libyen 
(J.L. Miège, 1975), laissant prendre la mesure de la centralité du Fezzan jusqu'aux premières années du $\mathrm{XX}^{\text {ème }}$ siècle. Mais le privilège est précaire car en l'espace de quelques années, l'extension de la domination coloniale sur le Sahara et la captation des courants commerciaux vers l'Afrique centrale et orientale, reliées aux réseaux ferrés, fluviaux et maritimes, précipitent le déclin du carrefour fezzanais.

La présence italienne dans le Fezzan est brève et marquée par l'opposition de la Senoussiya. En effet, si l'occupation de la Tripolitaine débute en 1911 et les principales villes fezzanaises, Mourzouq et Sebha, sont occupées en 1914, la même année, l'opposition de la Senoussiya contraint les occupants à quitter la région. Entre sa reconquête par les Italiens en 1930 et l'installation française de l'armée Leclerc en 1943, le Fezzan n'a donc été occupé qu'une dizaine d'années durant par le régime fasciste.

Cette occupation éphémère demeure uniquement militaire, contrairement aux provinces littorales pour lesquelles d'ambitieux programmes de peuplement et de mise en valeur agraire sont planifiés, et partiellement appliqués (F. Cresti, 1999). L'équipement de base des nouveaux villages littoraux coloniaux, recensé par Federico Cresti (1999), » bureaux de la municipalité et de la poste, la casa del Fascio (bureau du parti fasciste), le poste de police, l'église, l'école, l'infirmerie, des magasins et le marché couvert... », bref tout le nécessaire à la vie quotidienne d'une communauté italienne, ne se trouve dans le Fezzan qu'à Sebha et à Brak. Dans le Sahara, les meilleurs indicateurs de l'implantation italienne sont les aéroports, les réserves d'essence, les garages et les bureaux administratifs. Il faut dire que pour les Italiens, soucieux de s'établir sur la Quarta Sponda, la quatrième rive, le Fezzan, ne se réduit qu'à un hinterland de la Tripolitaine, une « modeste dot » (P. Soave, 2001) dont la crise économique et sociale, parallèle au déclin du commerce caravanier, s'amplifie après la première occupation italienne.

Les choix des autorités militaires se fondent donc sur la volonté de contrôler la circulation plutôt que les espaces oasiens, « de dominer plus que d'occuper » (A. Martel, 1991). Ce choix s'exprime à toutes les échelles spatiales puisque Sebha, jusqu'alors modeste relais secondaire sur les itinéraires caravaniers devient le centre principal de la présence coloniale italienne, au détriment de Mourzouq, capitale historique du Fezzan. Il est vrai qu' "occuper Sebha équivaut à occuper le Fezzan » puisque l'oasis « occupe une position centrale idéale sur les axes nord-sud venant du littoral... et sur la voie ouest-est, de Ghat vers l'Egypte » (Jacques Thiry, 1995). Dans les vallées, la même logique est appliquée : Al Gharayfa était 
le principal centre rural du Wadi Al Haya vers lequel convergeaient quelques axes de circulation de direction nord-sud et est-ouest et où se déroulait le plus important marché local. Mais c'est pourtant à Oubari que les autorités militaires installent une poste, un dispensaire, un terrain d'aviation, le siège de la circonscription Touareg et un bureau militaire. L'impératif stratégique prime encore car Oubari est le point de rencontre des Touaregs et des villageois du Fezzan. Les choix sont partout identiques : Brak et Edri, aux extrémités du Wadi Ash Shati sont préférés aux centres de Berqen et de Mahrouqa, de même qu'Oum Al Aranib à l'est de la dépression de Mourzouq.

Ces choix de site sont à l'origine des principales agglomérations du Fezzan actuel. Malgré une présence brève, les autorités coloniales italiennes inscrivent donc les prémices d'un marquage spatial où les lieux d'échanges, malmenés par le déclin du commerce transsaharien, sont remplacés par des lieux de contrôle des espaces et des circulations. En effectuant ce choix, les autorités militaires italiennes effacent les restes du réseau urbain et commerçant fezzanais mais dessinent une trame urbaine durable.

Lorsque Jean Lethielleux conclut le survol historique de son ouvrage consacré au Fezzan (1948) par une phrase lapidaire, « dès lors, le Fezzan n'a plus d'histoire » ou lorsque dans sa «Géographie Humaine » du Fezzan (1946), Jean Despois insiste sur l'état de «décadence» de la région, on devine le souci qu'ont ces deux auteurs d'y légitimer l'installation des Français. Il faut dire qu'en prenant pied dans le Fezzan en 1943, les troupes françaises matérialisent un projet colonial vieux d'un siècle, celui de constituer un vaste Sahara sous leur seule autorité. La défaite italienne leur en donne l'occasion puisque le territoire libyen est jusqu'à la fin du second conflit mondial provisoirement administré par les Britanniques, en Cyrénaïque et en Tripolitaine où ils composent avec les Italiens non fascistes, pendant que les Français obtiennent un mandat de l'ONU pour l'administration du Fezzan, prolongé après le renoncement officiel de l'Italie à toute prétention territoriale.

Dans la logique coloniale française, l'intérêt du Fezzan est évident puisqu'il permet d'achever la constitution d'un espace saharien cohérent, de dominer une région dont le sous-sol est susceptible de renfermer des ressources minières et en hydrocarbures et surtout de relier par l'itinéraire le plus court la Méditerranée à l'Afrique en ne survolant ou en ne traversant par la piste que les territoires sous domination française (A. Martel, 1991). Cet intérêt stratégique, doublé d'une fierté non dissimulée, conduit la France, par le biais de la Direction des Territoires du Sud, à 
convaincre les Nations Unies et les populations de la pertinence d'une intégration définitive du Fezzan au domaine colonial français. Dès lors, à partir d'un budget largement financé par le gouvernement français, les autorités militaires en charge du Fezzan multiplient les chantiers : forages de puits artésiens, création d'établissements d'enseignement primaire, rénovation du système de santé, prêt de semences, distribution de vêtements et de nourriture (DTS, 1953)... bref ce qui devait être réalisé en une génération... est mis aussitôt en chantier » (A. Martel, 1991).

Les options françaises sont pourtant en décalage avec la nouvelle géopolitique mondiale qui se dessine aux lendemains du second conflit mondial. Le mouvement de la décolonisation est déjà engagé en Asie et la naissance de l'Etat d'Israël incite la communauté internationale à accorder des compensations à la Ligue des Etats Arabes. Dans ce contexte, la Libye obtient son indépendance en 1951 et les Français quittent le Fezzan le $1^{\text {er }}$ janvier 1952.

Objet de convoitise durant plus d'un siècle, le carrefour fezzanais n'a jamais fait l'objet du moindre projet global de mise en valeur. La confrontation entre les acteurs de la conquête du Sahara déstructure définitivement la fonction séculaire de carrefour du Fezzan. De l'indépendance libyenne jusqu'aux années 1970, le Fezzan se réduit à la périphérie marginalisée d'un nouvel Etat dont l'un des objectifs majeurs est la construction d'un territoire national. 
CHAPITRE 2 - L'INTEGRATION DU FEZZAN AU TERRITOIRE NATIONAL

«A dater de ce jour, le Fezzan ne sera plus une terre oubliée ${ }^{11}$

Mouammar

Kadhafi

Peu ou prou, tous les dirigeants d'Etats sahariens ont aspiré à la mise en œuvre du projet d'intégration de la portion du Sahara sur laquelle ils exercent leur souveraineté au territoire national. Seule la Libye a réellement disposé, un temps, des moyens de ses ambitions faisant en moins de deux décennies du Fezzan l'un des exemples les plus aboutis de l'urbanisation de haut en bas. La hausse rapide des recettes liées à la vente des hydrocarbures fournit à l'Etat libyen des moyens qui décuplent de 1963 à 1969 , passant de 100 millions à 1.2 milliards de dollars et doublent durant la décennie 1970 pour s'effondrer à partir de 1981 (F . Burgat, A. Laronde, 1996). C'est donc dans le cadre d'une nationalisation progressive de l'appareil économique que le pouvoir concrétise ses ambitions.

C'est du «haut », l'Etat, vers le bas, «le local », qu'est impulsé le processus d'urbanisation et de modernisation, selon deux axes majeurs : l'extension des réseaux routiers, la distribution des fonctions commerciales, industrielles et administratives.

La conjonction de ces actions entraîne une multiplication rapide du nombre d'agglomérations. L'Etat les a créées afin de stabiliser et de regrouper les populations du Fezzan mais aussi avec l'objectif de mettre en œuvre le système de redistribution de la rente et de traitement égalitaire du territoire.

Le peuplement de ces agglomérations se diversifie progressivement. Elles croissent d'abord sous l'effet de l'exode rural depuis l'ensemble des espaces oasiens du Fezzan. Mais l'essor des migrations internationales transforme progressivement les principaux centres sahariens en creusets de population, mosaïques à l'image de ce qu'est le Sahara central contemporain.

${ }^{11}$ Citation tirée d'une brochure de présentation des projets de production céréalière du Fezzan 


\section{DÉSENCLAVEMENT ET MISE EN PLACE DE LA TRAME URBAINE}

Les étapes de la construction du réseau routier donnent une idée de la massivité de l'intervention étatique. 2000 kilomètres de routes sont construits entre 1970 et 1975,5000 entre 1980 et 1985 et seulement quelques centaines durant la décennie suivante. L'extension maximale s'effectue donc durant un laps de temps réduit, une dizaine d'années, puis le rythme des chantiers ralentit après l'effondrement des cours du baril de pétrole pour être interrompu lorsque, en 1987, le désengagement de l'Etat est officiellement annoncé.

Figure 4 : Le désenclavement du Sahara libyen

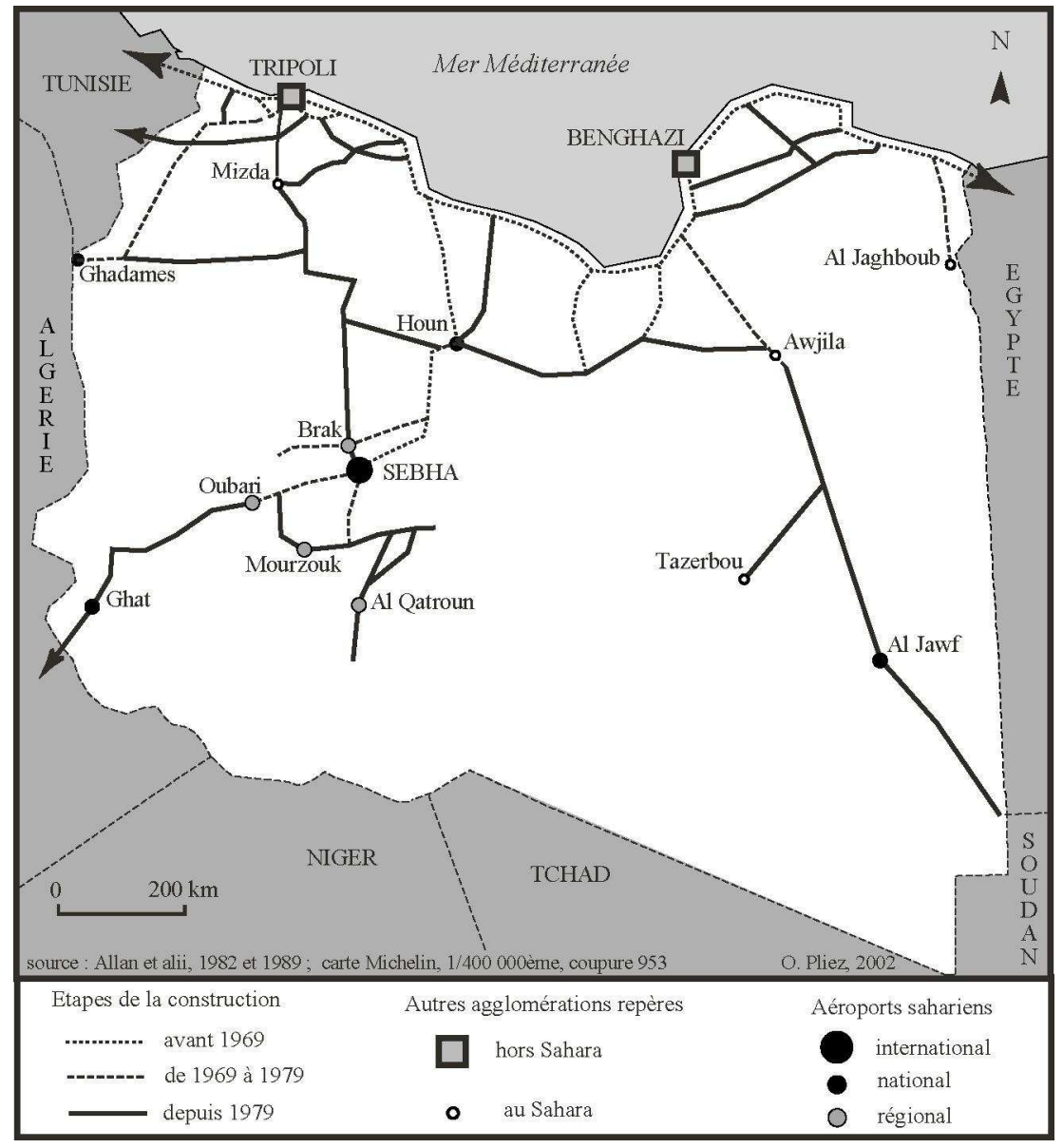


L'importance des travaux menés n'en est pas moins spectaculaire (fig.4). Sebha, Houn et les sites d'exploitation d'hydrocarbures sont reliés à l'axe central littoral durant les années 1960, première étape vers l'intégration des trois entités qui forment la Libye. Durant la décennie suivante, les agglomérations sahariennes «peu » éloignées du littoral de Ghadamès, Awjila et Al Jaghboub sont reliées au réseau routier national. Entre 1980 et 1985, les axes de Tripoli vers Sebha et Ghat et d'Awjila vers Al Jawf, dans le groupe d'oasis de Koufra sont achevés. Disposant de moyens financiers inégalés, l'Etat libyen achève ainsi l'essentiel du réseau routier national.

Dans toutes les directions, les axes goudronnés atteignent la limite méridionale des zones peuplées mais jamais les frontières. L'extension du réseau routier saharien apparaît donc d'abord comme un acte politique. Sa mise en œuvre aurait évidemment été difficile, et de toute évidence plus longue, sans les revenus exceptionnels tirés de la manne pétrolière. On comprend mieux que le recours au transport aérien ait souvent constitué un préalable pour les destinations les plus isolées, telles Ghadamès et les oasis de Koufra, à tel point qu'un cabinet d'experts internationaux, Doxyades, prônait en 1965 son extension au détriment de la route. L'importance et la qualité du réseau routier ont néanmoins eu un effet déterminant sur le processus d'urbanisation du Sahara libyen, d'autant qu'il fut parallèle à la rénovation rurale des régions d'oasis.

Parallèlement à la construction d'un réseau routier, l'Etat libyen procède à la réorganisation et à la densification de la trame urbaine en privilégiant trois objectifs : tenter de rééquilibrer le territoire national en limitant le poids des principales villes libyennes, Tripoli et Benghazi, qui concentrent une part croissante des habitants et des fonctions après l'accession à l'Indépendance, en impulsant la promotion administrative de centres régionaux et micro-régionaux sur l'ensemble du territoire ; diversifier les structures économiques des villes petites et moyennes; les doter en infrastructures de base, souvent de manière ambitieuse, dans les domaines de la santé, de l'éducation, des transports et des équipements (adduction d'eau et assainissement, électricité) (S.K. Qezeiri, 1986).

Dans un premier temps, cette politique conforte la hiérarchie urbaine du Fezzan selon une division en trois strates. Sebha voit son rôle de centre régional renforcé et est à ce titre dotée de fonctions administratives, économiques, universitaires et de transports importantes, quoique sans rapport avec celles des deux autres «capitales régionales » de Tripoli et Benghazi. Les centres principaux de chacun des quatre ensembles oasiens 
du Fezzan, Brak, Oubari, Mourzouq et Ghat, sont dotés de vastes périmètres agricoles, de services administratifs et sociaux (hôpitaux, banques), culturels et culturels (mosquées, bibliothèques, écoles et collèges). Tous les autres villages et bourgs bénéficient d'au moins une école, une poste, un souk d'Etat et une mosquée.

L'inventaire sommaire des fonctions dont chaque agglomération du Fezzan bénéficie laisse entrevoir l'artificialité d'une telle organisation. En effet, jusqu'à la moitié des années 1970 , la population active correspond à près d'un quart de la population totale dont $80 \%$ dans l'agriculture, $13 \%$ seulement dans l'industrie et le restant dans l'administration. La large majorité des agriculteurs occupe déjà un second emploi car leur activité principale ne leur permet pas de subvenir aux besoins du ménage, même si l'artisanat reste très répandu (M. Alawar, 1982). Or, à partir de la seconde moitié des années 1970, le processus d'urbanisation est mené parallèlement à la nationalisation des activités économiques. En conséquence, l'ensemble des agglomérations dépend des subsides de l'Etat, autant pour leur construction et leur équipement que pour la dotation en emplois de la population active. Or, le développement des secteurs productifs se limite souvent à la modernisation du secteur agricole et l'industrie se résume au développement du secteur de la construction et à la mise en place de quelques unités de production (céramique à Brak, pâtes alimentaires et boissons gazeuses à Sebha), dépendante de la maintenance de services techniques qui se trouvent au mieux à Tripoli, au pire hors de la Libye.

Le second temps de la politique de dotation en équipement pousse pourtant la logique de déconcentration plus avant. Ainsi, si logiquement, Sebha est dotée d'un aéroport international et Ghat d'un aéroport national, en revanche, Brak, Oubari et Mourzouq, pourtant proche, et même Al Qatroun, modeste bourgade excentrée, bénéficient de liaisons avec Sebha. C'est pourtant le système universitaire qui connaît la plus forte extension, étroitement corrélée à la poursuite de la promotion administrative de nouveaux centres. A Sebha se trouve la plus importante université, Oubari, Brak et Mourzouq sont équipées de centres notables, regroupant quelques milliers d'étudiants et durant les années 1990, presque toutes les bourgades exerçant quelque rayonnement à l'échelle d'une portion de vallée, comptent une antenne universitaire. Ainsi, actuellement, on ne dénombre pas moins de dix sites universitaires dans le Fezzan, qui sont pour la plupart d'entre eux très dégradés. En revanche, ils sont suffisamment proches de tous les foyers de peuplement pour que les étudiantes y soient aujourd'hui majoritaires. C'est là un fait essentiel car pour la plupart des familles du Fezzan, le fait que les filles puissent venir quotidiennement à 
l'université tout en demeurant chez elles, constitue la condition sine qua non d'accès à l'enseignement supérieur.

Créer des villes et les doter en équipement impose des choix et des arbitrages. En tentant de considérer le Sahara comme le reste du territoire national, les autorités libyennes ont mené à son terme leur vision de l'aménagement du territoire. C'est peut-être dans ce cadre que le traitement égalitaire du territoire et de ses habitants a montré ses limites avec plus de force que dans le reste de la Libye, la distance et la faiblesse des densités de peuplement rendant la maintenance d'un tel système plus difficile qu'ailleurs et par là plus sensible à la chute de la rente pétrolière.

\section{L'URBANISATION GÉNÉRALISÉE, UNE DYNAMIQUE EN DEUX TEMPS}

L'intensité de l'urbanisation se traduit par la croissance rapide du nombre d'agglomérations dans l'ensemble du Sahara (tab.2 et 3). Leur répartition s'explique différemment selon la période et la localisation. En 1973, on ne dénombre que 26 agglomérations dans l'ensemble du Sahara libyen mais en 11 ans, jusqu'au recensement suivant, leur nombre est multiplié par deux et demi. Il n'évolue guère par la suite, passant de 66 en 1984 à 77 en 1995. La multiplication rapide du nombre d'agglomérations confirme donc la relation étroite entre l'action de l'Etat et l'urbanisation en même temps que sa brièveté.

Tableau 2 : Nombre d'agglomérations par strate (1973-1984-1995)

\begin{tabular}{|l|c|c|c|c|c|c|}
\hline & \multicolumn{2}{|c|}{1973} & \multicolumn{2}{c|}{1984} & \multicolumn{2}{|c|}{1995} \\
\hline & Total & $\begin{array}{c}\text { dont } \\
\text { Fezzan }\end{array}$ & Total & $\begin{array}{c}\text { dont } \\
\text { Fezzan }\end{array}$ & Total & $\begin{array}{c}\text { dont } \\
\text { Fezzan }\end{array}$ \\
\hline $\begin{array}{l}\text { De 1500 à } \\
\text { 3500 }\end{array}$ & 18 & 11 & 50 & 30 & 41 & 27 \\
\hline $\begin{array}{l}\text { De 3500 à } \\
\text { 6000 }\end{array}$ & 5 & 1 & 6 & 5 & 21 & 15 \\
\hline $\begin{array}{l}\text { De 6000 à } \\
\text { 10000 }\end{array}$ & 2 & - & 6 & 1 & 5 & 2 \\
\hline $\begin{array}{l}\text { De 10000 à } \\
\text { 30000 }\end{array}$ & - & - & 3 & 1 & 8 & 2 \\
\hline Plus de 30000 & 1 & 1 & 1 & 1 & 1 & 1 \\
\hline Total & 26 & 13 & 66 & 38 & 76 & 47 \\
\hline
\end{tabular}

Source : RGP 1973, 1984, 1995 
Dès 1973 (fig.5), la trame urbaine du Sahara libyen diffère nettement de celle du reste du Sahara maghrébin. Du Maroc à la Tunisie, on observe vis-à-vis des régions sahariennes la persistance d'un gradient nord-sud marqué. L'urbanisation y est intense sur les piémonts puis elle devient discontinue au fur et à mesure que l'on s'avance vers les parties méridionales des territoires nationaux. Elle se confond alors avec les sites des régions d'oasis anciennes. En Libye, au contraire, l'absence d'une limite montagnarde aussi marquée entre le littoral et le désert a limité les effets de gradient. Le Fezzan, composante historique de la Libye, concentre, par contre, aujourd'hui la plus importante part de ces agglomérations. Une structure de peuplement ancienne se conforte donc.

Entre 1973 et 1984, les réseaux s'étoffent avec la multiplication des petits centres liés à la fixation des nomades et à la croissance démographique. La fixation est particulièrement active entre Ghadamès et Nalout au nord-ouest, entre Mizda et la Joufra au centre-ouest, le long des marges méridionales avec les villages d'Al Awaynat et Al Qatroun et dans la dépression de Mourzouq, notamment autour de Tsawa. Dans les principales régions oasiennes, le Fezzan, la Joufra, Koufra et Jalou, tous les villages connaissent des taux d'accroissement naturel élevés auxquels s'ajoute l'installation d'immigrés sur l'ensemble du territoire national.

Tableau 3 : Mesures de l'urbanisation dans le Sahara libyen

\begin{tabular}{|c|c|c|c|c|c|}
\hline & \multicolumn{3}{|c|}{ Recensements } & \multicolumn{2}{|c|}{$\begin{array}{c}\text { Croissance annuelle } \\
\text { (en \%) }\end{array}$} \\
\hline & 1973 & 1984 & 1995 & 1973-1984 & 1984-1995 \\
\hline Population agglomérée* & 111062 & 291492 & 445475 & 9.2 & 4 \\
\hline dont Fezzan & 87354 & 181623 & 268440 & & \\
\hline Population totale & 172756 & 324795 & 486378 & 5.9 & 3.7 \\
\hline dont Fezzan & 111349 & 208313 & 310992 & & \\
\hline Taux d'urbanisation & 64.3 & 89.7 & 91.5 & & \\
\hline \begin{tabular}{|ll} 
Fezzan & Tx urb. \\
\end{tabular} & 78.5 & 87.2 & 86.3 & & \\
\hline \begin{tabular}{|l|} 
Nombre \\
d'agglomérations
\end{tabular} & 26 & 66 & 76 & & \\
\hline
\end{tabular}

Entre 1984 et 1995 (fig.5), les réseaux se stabilisent, quoique très inégalement. En effet, sur les dix nouveaux centres émergeants, neuf se situent dans le Fezzan. Leur multiplication est donc essentiellement fonction de la densité du réseau villageois initial. Dans la plupart des 
ensembles oasiens, les agglomérations comptent désormais plus de 1500 habitants ou bien ont totalement périclité, ce qui est loin d'être le cas au Fezzan. Mais plus que les taux de croissance, la multiplication du nombre d'agglomérations est un indicateur de tendance pertinent.

Figure 5 : Les agglomérations du Sahara libyen en 1973

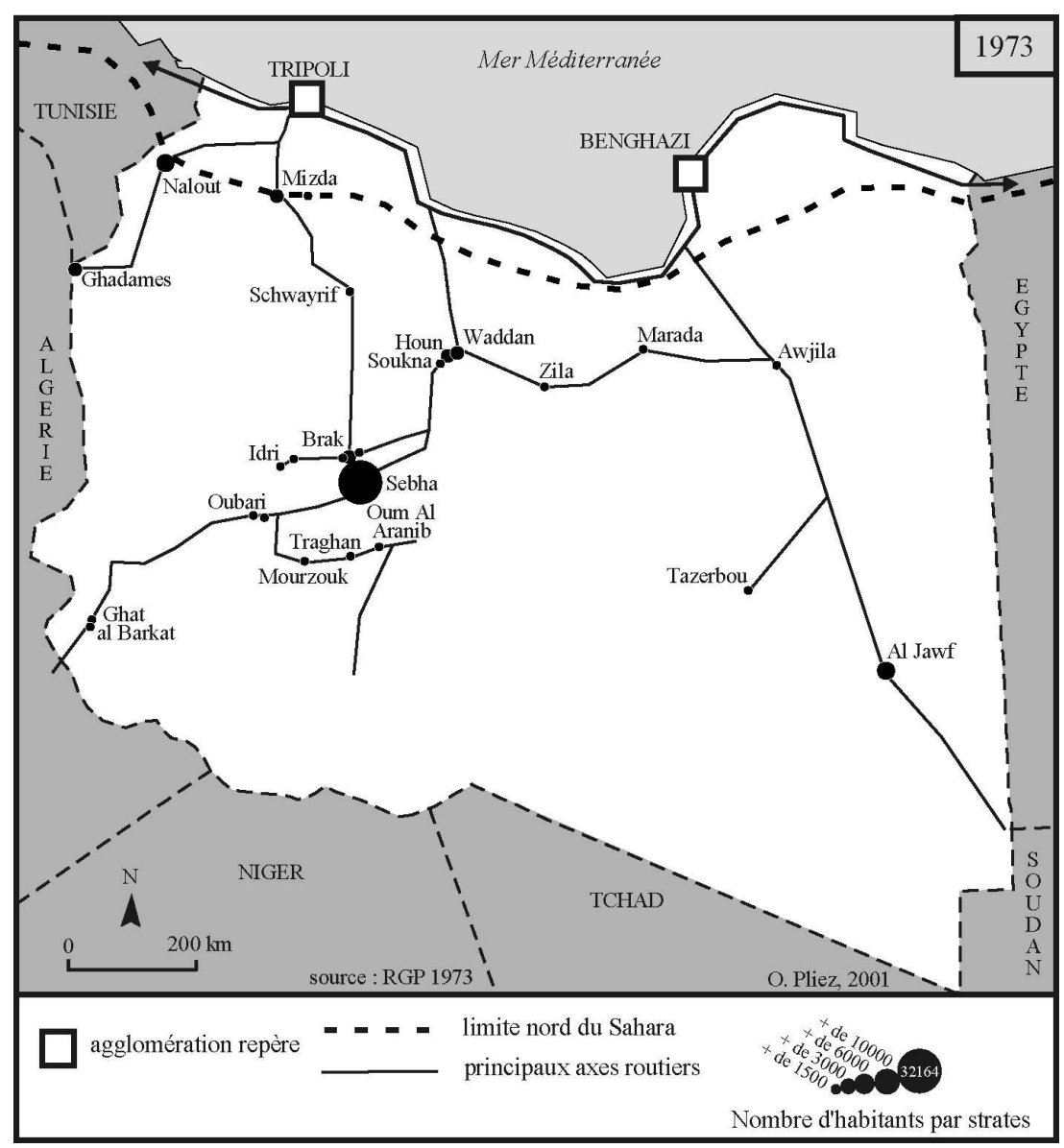

Une évidence s'impose, l'urbanisation du Sahara libyen est une micro-urbanisation. En effet, en 1995, une agglomération sur deux compte moins de 3500 habitants et huit sur dix moins de 6000 . En conséquence, la multiplication du nombre d'agglomérations durant les années 1970 et 1980 est avant tout celle des petits centres dont la répartition reflète le tropisme des axes routiers. Au recensement de 1995, le système urbain du Fezzan 
restitue une image paradoxale puisque quatre personnes sur dix vivent dans une agglomération, Sebha, et cinq sur dix dans un semis régional composé de plus de quarante centres. Le réseau urbain fezzanais, à la fois macrocéphale et éclaté, permet de rappeler que ce que l'on considère comme un déséquilibre ailleurs est ici la norme. C'est sans doute l'une des plus fortes spécificités des systèmes urbains sahariens actuels.

Figure 6 : Les agglomérations du Sahara libyen en 1995

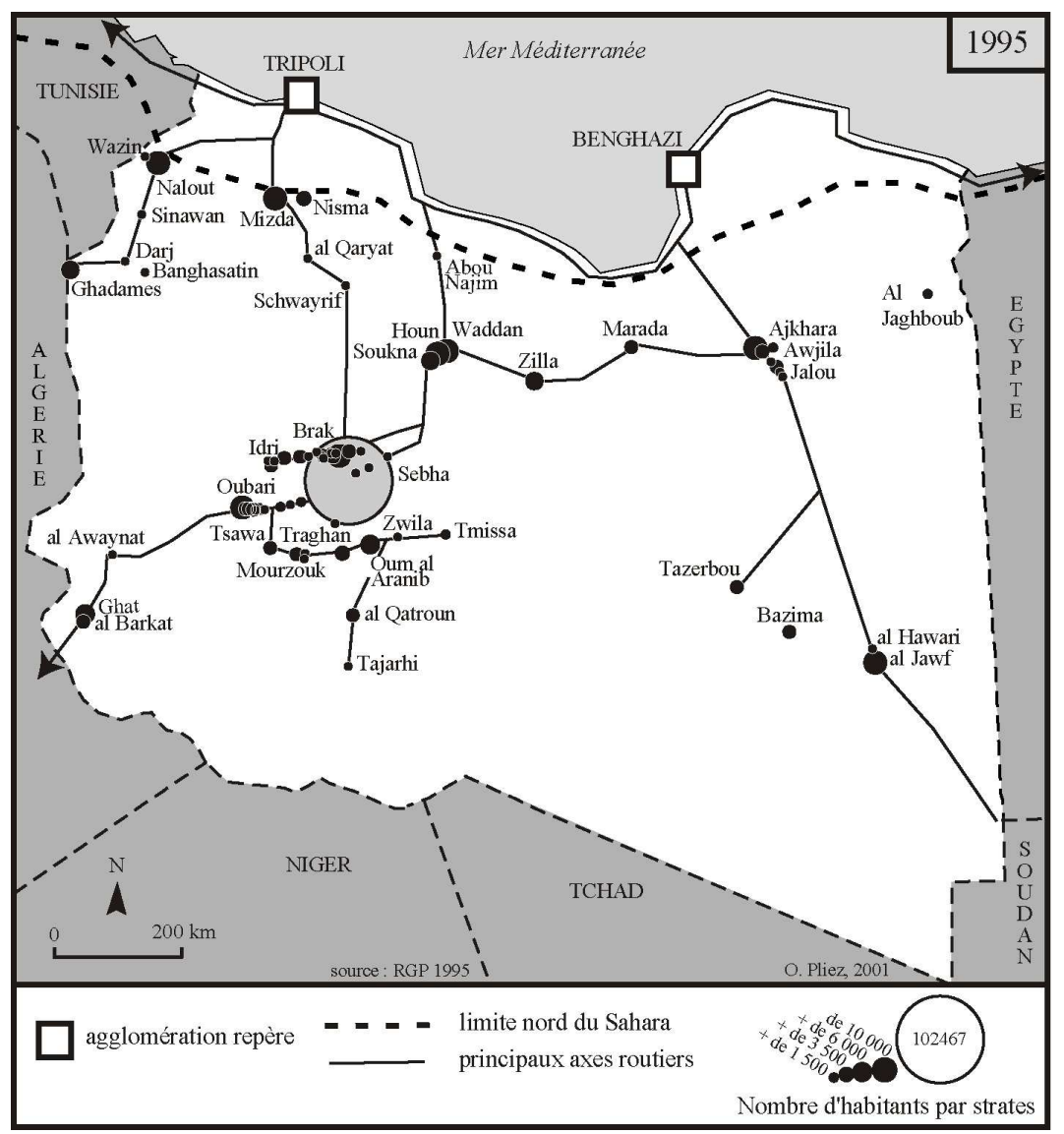

En fin de compte, l'urbanisation n'est pas en Libye de nature à remettre en cause la répartition de la population saharienne. Les dissymétries fondamentales, entre l'Est et l'Ouest et entre le Nord et le Sud du Sahara persistent. Les totaux de population viennent en appui de ce constat avec une remarquable constance, puisque la part du Fezzan dans la population 
totale du Sahara libyen oscille de 63 à $64 \%$ entre 1973 et 1995 . Bel exemple de l'inertie qu'un système de peuplement ancien oppose au volontarisme étatique même si c'est à l'initiative de l'Etat que le Sahara sort d'une longue période de « décadence».

\section{LES MIGRATIONS INTERNES, PRODUITS ET RESSORTS DE LA CROISSANCE URBAINE}

La mobilité des populations sahariennes n'a évidemment rien de nouveau. En revanche, l'établissement des frontières a provoqué la dissociation de ces mobilités entre mouvements internes ${ }^{12}$ et internationaux. L'intervention de l'Etat-aménageur constitue là aussi une rupture car il a contribué à accélérer et à orienter les flux de migrants au fur et à mesure de l'intégration du Sahara au territoire national. Le façonnement du cadre national par le haut s'accompagne donc d'une redistribution interne des populations et d'une adéquation croissante avec le processus d'urbanisation.

La tendance, entre 1964 et 1995, est à la réduction progressive des migrations. $10 \%$ des Libyens changent de lieu de résidence entre 1964 et 1973 (150 000 personnes), $5 \%$ entre 1973 et 1984 (112 000 personnes) et 2 $\%$ entre 1984 et 1995 (67000 personnes). Cette décroissance s'observe dans l'ensemble de la Libye mais selon des nuances sensibles, notamment dans le Fezzan où le taux est systématiquement plus élevé puisqu'il chute entre chaque période intercensitaire de $28 \%$ à $14.8 \%$ puis à $6.6 \%$. Dans un premier temps (fig.7), seules les deux « capitales », Tripoli et Benghazi enregistrent des soldes migratoires positifs, attirant des Libyens de toutes les régions mais dès la période 1973-1984, la plupart des régions sahariennes connaissent à leur tour des soldes positifs. Par la suite, la situation s'inverse puisque Tripoli et Benghazi enregistrent des soldes négatifs alors que des régions jusque-là déficitaires connaissent un renversement de tendance.

Jusqu'aux années 1950, l'émigration depuis le Fezzan est importante et généralisée. A Ghat et à Mourzouq, un habitant sur cinq a émigré

\footnotetext{
${ }^{12}$ Afin de mesurer les migrations internes, nous avons utilisé, à la suite de D. Retaillé (1995) pour la Mauritanie, le coefficient de préférence élaboré par P. Merlin dans Géographie de l'aménagement, PUF, 1988, pp.74-76. Ce coefficient présente l'intérêt d'effacer les effets de masse entre les régions d'émigration et d'immigration et donc de procurer une information fine sur les flux préférentiels depuis ou vers une région donnée. L'attraction est d'autant plus sélective qu'elle est supérieure à 1 et répulsive qu'elle est inférieure à 1 .
} 
temporairement ou définitivement (L. Eldblom, 1968). Sebha capte déjà la moitié des flux depuis ces agglomérations car elle cumule les fonctions de viviers d'emplois saisonniers et d'étape entre le Fezzan et le littoral. Durant les années 1960, alors que le Fezzan enregistre un solde migratoire négatif vis-à-vis du reste de la Libye (Atlas National, 1979), Sebha accroît son attraction en accueillant les deux-tiers des flux régionaux d'émigration, Brak, Oubari et Mourzouq, le tiers restant mais 9000 fezzanais sont installés à Tripoli et à Benghazi (M. Alawar, 1982).

Après 1973, l'attractivité de Sebha, surtout régionale, tend de plus en plus à rayonner au-delà des foyers de peuplement alentours, entraînant la diversité croissante des populations qui s'y installent. Les migrations internes y contribuent pour moitié à la croissance démographique entre 1973 et 1984 contre à peine plus d'un dixième à Oubari.

Entre 1984 et 1995, pour la première fois, le solde migratoire de Sebha serait négatif sans l'installation des Libyens de l'extérieur, principalement vis-à-vis des baladiyat environnantes. Sebha connaît donc une évolution identique à celle de Tripoli et Benghazi : de pôle d'abord attractif, la ville devient ensuite répulsive au fur et à mesure de l'équipement et de l'urbanisation du reste du territoire qui permet aux populations fezzanaises de ne plus quitter leur localité voire d'y revenir. Toutefois, à Oubari comme à Sebha, si plus de la moitié des migrants venaient du reste de la région en 1973, un flux de population notable depuis Sirte vers Sebha et depuis Ghadamès vers Oubari se maintient. La relation avec les espaces nomades s'affirme avec évidence puisque la baladiya de Sirte recoupe les territoires des Ouled Sliman, des Riah, des Guededfa et des Ouafi (J. Despois, 1946), traditionnellement en contact avec le Nord et l'Est du Fezzan et la baladiya de Ghadamès fait partie du même espace nomade qu'Oubari. La fixation des nomades s'est donc accompagnée de regroupements familiaux. De plus, la part des Libyens qui résidaient auparavant à l'étranger croît très rapidement en direction de Sebha et tend même à s'amplifier d'un recensement à l'autre.

Durant les années 1990, la transition urbaine du Sahara libyen s'achève. En moins de trois décennies, les agglomérations se sont multipliées comme autant de repères, voire de marqueurs, d'un territoire national en voie de constitution. Quoique récentes, ces agglomérations s'inscrivent dans une trame oasienne ancienne, devenue «territoire sans villes» durant la première moitié du $\mathrm{XX}^{\text {ème }}$ siècle. Le Fezzan constitue un cas à part à l'échelle du Sahara puisque les trois vallées qui le composent forment un réseau urbain d'une densité et d'une variété exceptionnelles. En Libye, l'urbanisation rapide et généralisée du Sahara est le fruit de la volonté des 
autorités publiques et des moyens financiers dont elles ont disposés. Mais on ne peut dissocier cet acte de son contexte : pour les autorités, urbaniser, c'est atténuer le tropisme exercé par les pôles littoraux auprès des populations sahariennes et exploiter des potentialités économiques jusque-là négligées.

L'exemple du Fezzan permet d'illustrer ce qui constitue souvent pour l'observateur extérieur un paradoxe. L'exploitation saharienne des hydrocarbures a, en définitive, peu d'effets urbanisants dans la région d'extraction. En revanche, les revenus qu'il procure permettent d'assurer la pérennité des véritables foyers de peuplement.

Pour l'essentiel, les modalités selon lesquelles l'Etat libyen a mis en œuvre l'intégration du Fezzan au territoire national ne diffèrent pas dans les objectifs mais plutôt dans les moyens avec le reste du Maghreb. Néanmoins, des années 1950 aux années 1970, l'émigration liée à la crise économique et sociale du Sud devient, au fur et à mesure, exode rural dans un cadre d'abord national mais de plus en plus nettement régional au fur et à mesure de la progression du processus d'urbanisation saharien. Il apparaît désormais que la centralité de Sebha n'est plus seulement régionale mais aussi « internationale » comme l'atteste la poursuite des flux de rapatriés libyens. Paradoxalement, alors que la multiplication des agglomérations matérialise l'intégration nationale, leur peuplement révèle et fonde les réseaux sahariens dans lesquels elles s'insèrent. 
Figure 7 : Les migrations internes en Libye de 1973 à 1995

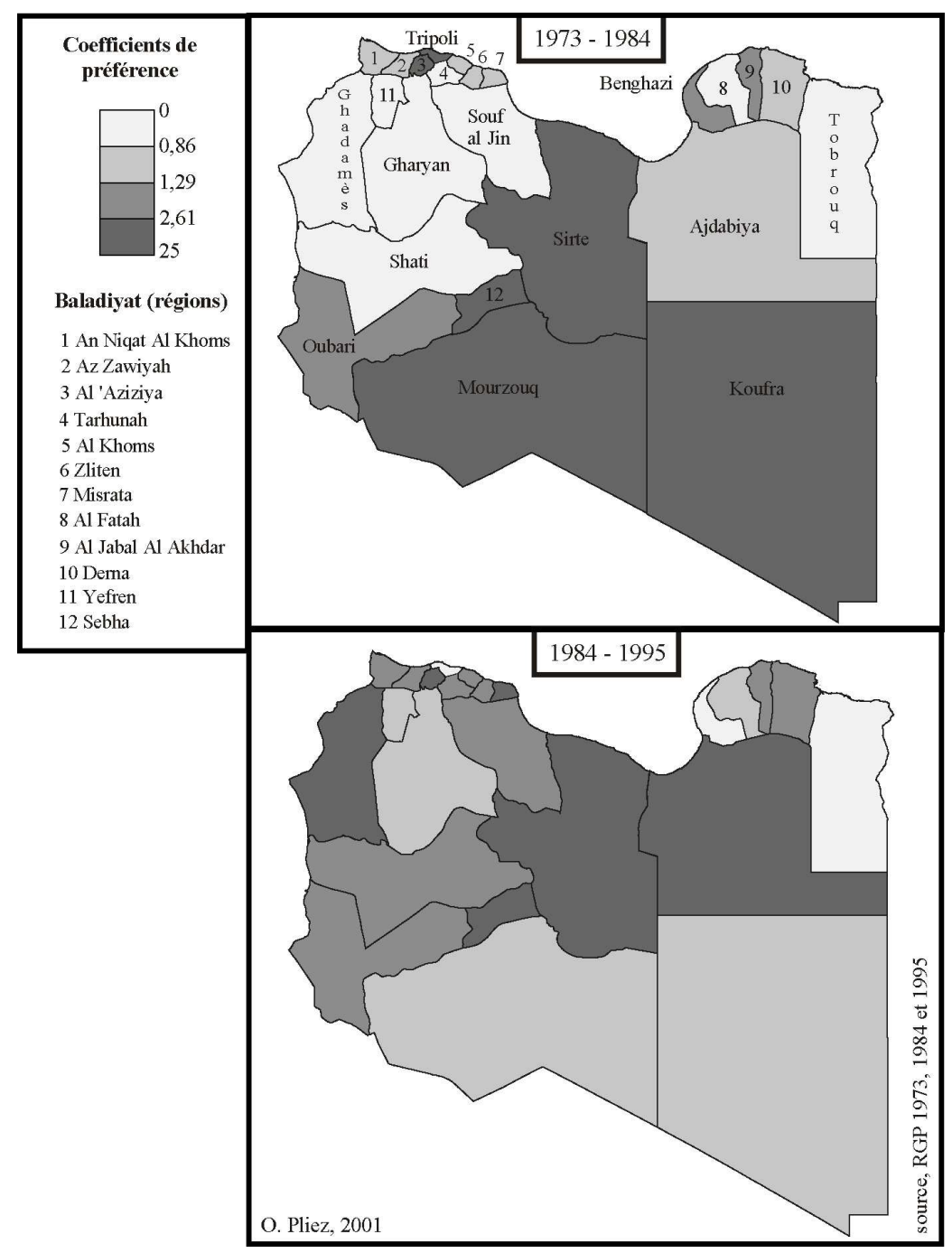




\section{CHAPITRE 3 - LE RENOUVEAU DU CARREFOUR FEZZANAIS}

La politique libyenne d'ancrage du Fezzan au territoire national est désormais achevée. Pour autant, le profond remodelage spatial qui l'a accompagnée n'a contribué qu'un temps au cloisonnement du Sahara libyen. Les indices de la réactivation d'un espace de circulation migratoire et marchand saharien se multiplient actuellement entre la Libye et l'Afrique subsaharienne.

Récente dans ses formes, la circulation n'en reste pas moins inscrite dans une continuité historique puisque les réseaux d'aujourd'hui se calquent sur ceux que les caravanes empruntaient hier, même si l'évocation de ce passé prestigieux ne suffit pas à tout comprendre. En revanche, les guerres, les périodes de sécheresses ou les insurrections qui ont secoué le Sahara central durant le $\mathrm{XX}^{\text {ème }}$ siècle, ont contribué à disperser les populations qui les ont subies. Et paradoxalement, ces crises, en divisant les familles et les groupes tribaux entre différents Etats, sont à la base d'actifs réseaux transfrontaliers qui lient le Fezzan aux régions sahariennes des pays voisins.

En outre, la nouvelle donne géopolitique entre la Libye et l'Afrique subsaharienne, voire entre l'Europe et l'Afrique, contribue désormais à conférer au Fezzan une position de carrefour au rayonnement allant très au-delà des limites du Sahara.

Or ces circulations se déploient dans un espace récemment remodelé par l'Etat libyen. Les villes d'Etat, en devenant carrefours sahariens, se transforment rapidement. La circulation des personnes en fait des lieux cosmopolites et contribue à l'extension spatiale des tissus urbains. La circulation des marchandises les transforme en places marchandes. Progressivement, le lien que l'Etat a voulu exclusif avec les communautés locales du Fezzan se distend au profit du renouveau du carrefour fezzanais.

\section{UN ESPACE MIGRATOIRE DE PROXIMITÉ}

Durant le $\mathrm{XX}^{\text {ème }}$ siècle, trois flux migratoires ont contribué à la construction d'un espace migratoire de proximité, intra-saharien, par-delà les limites entre les Etats voisins de la Libye, du Niger et du Tchad.

Les opposants à la colonisation avaient quitté la Libye au fur et à mesure de l'avancée italienne, s'installant dans les Etats voisins, notamment en Tunisie, en Egypte et au Tchad. Dès l'Indépendance, un 
grand mouvement de retour des «rapatriés » s'enclenche. En 1963, ils étaient estimés à 54000 , soit $4 \%$ de la population totale ; entre 1973 et 1984 , ils composent $16 \%$ des migrants internes (18 000) et encore $12 \%$ entre 1984 et 1995 (8 000).

Durant les années 1950 et 1960, les deux capitales, Tripoli et Benghazi, sont les destinations privilégiées des rapatriés. Mais dès les années 1970, un recentrage très net s'opère vers les baladiyat sahariennes et orientales. La référence historique est évidente puisqu'il s'agit des régions où l'opposition à la colonisation a été la plus violente et la plus longue, celles où la confrérie de la Senoussiya était la mieux implantée. Sebha tient une place à part, captant un cinquième puis plus de la moitié des arrivées totales de rapatriés pour les périodes intercensitaires de référence, soit les deux tiers des arrivées de Libyens vers la ville entre 1984 et 1995.

Il paraît a priori étonnant que des flux de rapatriés s'étalent sur plusieurs décennies, laissant supposer que ceux qui rentrent en Libye sont les descendants de ceux qui l'ont quittée. Des itinéraires familiaux permettent de comprendre la dimension saharienne de ces migrations :

Opposants aux autorités coloniales italiennes, des familles entières de la puissante tribu des Ouled Sliman fuient vers le Sahara algérien, nigérien et surtout tchadien où ils peuvent compter sur les réseaux de la Senoussiya. De là, en deux générations, des familles optent, volontairement ou sous la contrainte, pour des itinéraires très diversifiés. Certains s'y installent, investissent dans le secteur commercial à Faya, Abéché ou dans le Kanem, d'autres étendent les réseaux commerçants dans lesquels ils sont impliqués vers le Cameroun et la République centrafricaine, voire émigrent en France. Les autorités libyennes incitent ceux qui le désirent à revenir s'installer en Libye, principalement dans le Sahara, en leur proposant une maison, un emploi et une somme financière conséquente. Ainsi, en trois générations, les Ouled Sliman, à partir d'un ancrage fezzanais tissent des réseaux dans un espace qui s'étend de l'Europe à l'Afrique centrale. 
Cette dispersion, commune à de nombreuses familles de rapatriés, est devenue progressivement «ressource spatiale» ${ }^{13}$. Des réseaux commerçants transsahariens se mettent en place, entretenus par des alliances matrimoniales et les déplacements d'un lieu à l'autre. Ainsi, les Libyens qui rentrent encore en Libye, de plus en plus vers les régions sahariennes, sont bien souvent les enfants ou les petits-enfants de ceux qui étaient partis durant les années 1930. Nés à plusieurs centaines, voire plusieurs milliers de kilomètres de leur « terre natale », ils s'y installent, riches de leur réseau familial et souvent aussi commerçant.

Longtemps région d'émigration, le Fezzan attire aussi des immigrants. Cela peut ne pas surprendre compte tenu de l'attractivité de la Libye mais il nous importe ici de voir selon quelles modalités s'est mise en place cette facette de l'espace migratoire de proximité.

Jusqu'aux années 1960, les migrations pastorales et commerciales des nomades toubous du Tibesti au Tchad étaient actives mais la concurrence du camion, qui transporte bétail et marchandises depuis la Libye, provoque leur déclin (R. Capot-Rey, 1973). Les Tedas (groupe toubou du Tibesti) émigrent alors vers la Libye afin de travailler sur les chantiers ou les sites d'exploitation des hydrocarbures durant quelques mois; ce mouvement concerne un quart de la population mâle en âge de travailler en 1964. La crise climatique de 1969 à 1974 frappe durement le cheptel des Toubous Daza du Kanem, dans les terres basses situées entre le sud du Tibesti et le nord du Lac Tchad. Un flux d'émigration s'enclenche à destination du Fezzan et des villes du littoral libyen qui concerne, selon les comptages partiels, la moitié des chefs de famille entre 1972 et 1979 (J.C. Clanet, 1981). L'aire d'émigration touboue s'étend donc vers le Sud.

Ces deux exemples, quoique circonscrits au plan spatial, permettent de distinguer différents types de mobilités. A plusieurs reprises, Jean-Charles Clanet souligne la familiarité des itinéraires et des lieux choisis par les Daza candidats à l'émigration vers la Libye. Cette familiarité puise dans la proximité et dans l'histoire. En effet, et on recoupe ainsi les espaces nomades, les Toubous sont présents dans le Sahara libyen, notamment à Koufra et dans le sud du Fezzan, et y circulent depuis plusieurs siècles ; ce constat vaut aussi pour les Touaregs. Par conséquent, les fuseaux nomades de circulation dans lesquels se sont inscrits les échanges transsahariens sont aussi ceux dans lesquels se fixent les nomades et le long desquels se déplacent migrants et réfugiés aujourd'hui.

${ }^{13}$ MA MUNG (E.), «La dispersion comme ressource spatiale », Cultures et Conflits, Les anonymes de la mondialisation, $\mathrm{n}^{\circ} 33-34,1999$, pp. 89-103. 
La fixation des nomades est l'un des moteurs essentiels de l'urbanisation saharienne, particulièrement intense lors des «crises du pastoralisme » que les pasteurs des marges sahariennes ont subies (A. Bourgeot, 1995). Mais ce mouvement particulièrement marqué à la charnière Sahara-Sahel, ne suffit pas à expliquer les rapports entre la circulation de proximité et l'urbanisation dans les fuseaux nomades. Aux marges du Fezzan, les espaces du nomadisme s'étendent bien au-delà des frontières, vers le Niger et le Mali dans le cas des Touaregs, vers l'ouest du Niger et le Tchad dans le cas des Toubous. Ce décalage est à l'origine de deux types de circulations difficiles à dissocier l'un de l'autre. En incitant les nomades à se fixer, les autorités libyennes ont visé ceux qui vivaient en Libye mais le mouvement s'est étendu au-delà des frontières. Il s'avère donc parfois difficile de dissocier l'immigration de la fixation des nomades, en particulier dans les petites agglomérations. Ainsi, la quasi-totalité de la population d'Al Barkat à Oubari est composée de Touaregs, nés en Libye, au Niger ou en Algérie et dans la région d'Al Qatroun, l'essentiel des résidents sont Toubous, libyens, tchadiens ou nigériens.

Le premier type de circulation s'effectue sur les parcours et dans le cadre d'une «transaction» avec l'Etat libyen qui incite à la fixation définitive des nomades tout en facilitant cette installation ${ }^{14}$ car en général, la fixation des nomades s'accompagne de la création d'un village et d'un projet agricole comme à Maknoussa et à Tsawa, à l'est de Mourzouq, et le long de l'axe routier entre $\mathrm{Al}$ Awaynat et $\mathrm{Al}$ Barkat. Au milieu des années 1980, l'arrivée massive de réfugiés Toubous et Touaregs touche à nouveau les mêmes populations mais selon des modalités différentes. Lors de la guerre entre la Libye et le Tchad entre 1973 et 1994, l'armée libyenne annexe les provinces du nord du Tchad, le Borkou, l'Ennedi et le Tibesti (BET). Des cartes d'identité libyennes sont distribuées aux populations et le dinar libyen devient la monnaie d'échange (K. Bennafla, 1999). A la suite des revers subis par l'armée libyenne durant les années 1980, des Toubous fuient la région et s'installent dans le Sud libyen, notamment à Sebha et à $\mathrm{Al}$ Jawf (Koufra). L'arrivée des Touaregs, dans leur majorité nigériens, correspond à la conjonction de plusieurs facteurs, la succession des phases de sécheresses, les insurrections vis-à-vis du pouvoir central et l'activisme de Mouammar Kadhafi qui tente de déstabiliser le gouvernement nigérien (1983) et accueille les réfugiés Touaregs fuyant la répression. En l'espace de quelques années, une «diaspora en

\footnotetext{
${ }^{14}$ Il ne faudrait évidemment pas en conclure hâtivement que cette « transaction » soit toujours engagée entre pouvoirs publics et groupes nomades. Mais il paraît tout aussi utile de souligner que la fixation n'est pas systématiquement un acte autoritaire et subi.
} 
communautés » (A. Bourgeot, 1995) se déploie dans les régions sahariennes transfrontalières de l'Algérie et de la Libye. Certains rejoignent leurs familles vivant dans ces Etats mais beaucoup sont installés dans des camps frontaliers ${ }^{15}$ ou bien directement en périphérie des principales villes, Ghat, Oubari, Mourzouq et Sebha, nourrissant ainsi la croissance urbaine spectaculaire du Sahara central.

La dispersion des populations est donc une donnée essentielle dans le Sahara contemporain. Parfois, elle s'atténue, avec le retour des rapatriés libyens, d'autre fois, elle s'accroît, avec les déplacements de réfugiés, et dans tous les cas, elle contribue à ce que les membres des différentes communautés tissent des liens familiaux, tribaux et commerciaux inscrits dans la durée par-delà les frontières. Ces réseaux, structurés à partir des principales agglomérations et des groupes d'oasis, sont donc familiers, mais pas pour autant dépourvus de risques, à une partie de ceux qui les empruntent. Ils sont pour l'essentiel la conséquence d'une mobilité de proximité des Arabes, des Touaregs et des Toubous vivants dans ces régions transfrontalières, recoupant les fuseaux nomades qui structurent le Sahara central.

\section{UN « ENTRE-DEUX » ENTRE L'AFRIQUE ET LE MAGHREB}

En devenant une charnière entre l'Afrique subsaharienne et le Maghreb, le Fezzan renoue avec une fonction ancienne. Toutefois, un changement d'échelle est patent car les flux à destination, ou via, le Sahara libyen sortent de plus en plus nettement des limites du Sahara. Les facteurs qui contribuent à cette mutation relèvent de la géopolitique interne autant qu'externe de la Libye.

L'importance de l'immigration vers la Libye est connue, favorisée par la faiblesse du peuplement, la concentration des nationaux dans la fonction publique ou la multiplication des emplois liés à l'exploitation des hydrocarbures. Depuis les années 1970, des centaines de milliers de travailleurs venaient travailler en Libye, principalement depuis le Monde arabe, aux premiers rangs desquels les Egyptiens et les Tunisiens. Or depuis le milieu des années 1990, un basculement des flux migratoires est en cours, caractérisé par la croissance rapide du nombre de ressortissants d'Afrique subsaharienne.

\footnotetext{
${ }^{15}$ En Algérie: In Guezzam, Bordj El Mokhtar, Tin Zawaten, Timiawin, Adrar, Tamanghasset et en Libye : Oubari, Ghat, Ghadamès, Sebha.
} 
Ce basculement s'explique par la conjoncture politique et les choix libyens qui en découlent. Le 31 mars 1992, le conseil de sécurité des Nations Unies impose un embargo militaire et aérien à la Libye après que les services secrets libyens sont accusés d'avoir pris part à l'explosion d'un appareil américain de la TWA au-dessus de Lockerbie, en Ecosse, et d'un avion français d'UTA au-dessus du Niger.

\section{Photographie 1 : Camion à destination du Niger (Sebha)}

Les passagers (40 à 50 personnes) en attente du départ s'installent au-dessus de leurs bagages et des marchandises que transportent des convoyeurs durant un trajet éprouvant et périlleux de plusieurs jours qui les mène à Dirkou (Niger) et de là à N'Djamena (Tchad) ou à Agadès (Niger).

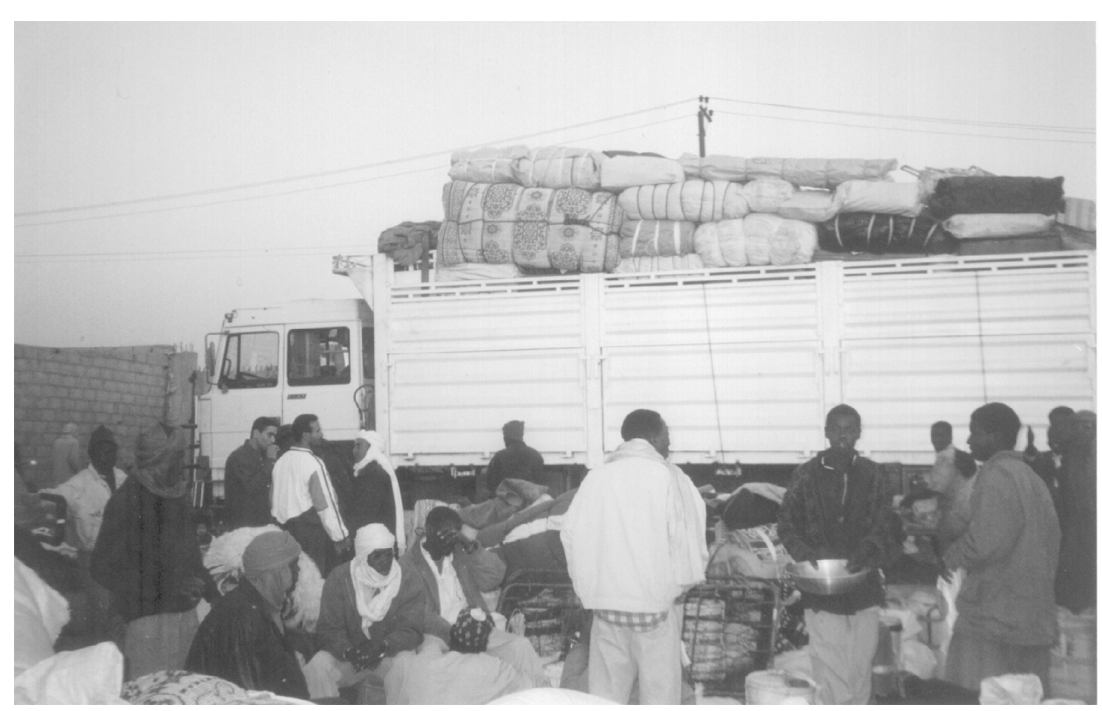

Mouammar Kadhafi lance de nouvelles initiatives pour briser l'isolement, charte d'intégration avec le Soudan en 1990 (F. Burgat, A. Laronde, 1996), accord de séjour et de libre passage des personnes avec le Tchad en 1994, création de la Communauté des Etats sahélo-sahariens (COMESSA ou CENSAD) en 1998. Ce grand projet «kadhafien», financé pour près de la moitié par la Libye, qui se veut la première étape vers les Etats-Unis d'Afrique regroupe six Etats à sa création (Burkina-Faso, Mali, Niger, Soudan, Tchad), onze en 2000 (Djibouti, Erythrée, Gambie, République centrafricaine, Sénégal) et seize en 2001 (Egypte, Maroc, Nigéria, Somalie et Tunisie). En se donnant comme objectif d'établir la libre circulation des personnes, des capitaux et la 
liberté de résider, travailler ou acquérir des biens, les Etats membres du CENSAD créent un cadre institutionnel facilitant les mobilités.

Toutefois, ces flux dépassent rapidement les limites du groupement inter-étatique car le prosélytisme dont fait preuve le Guide libyen et la médiatisation à laquelle il se livre dépassent rapidement les limites du CENSAD pour s'adresser à tous les candidats à l'émigration du continent africain. Ces appels reçoivent un écho d'autant plus favorable qu'ils interviennent dans un contexte de durcissement des conditions d'accès à l'Europe occidentale. En réponse, les filières migratoires et les itinéraires empruntés par un nombre croissant d'Africains se diversifient. Ils tentent de se rendre en Europe en empruntant les trajets terrestres sahariens via la Libye et l'Algérie puis le Maroc et la Tunisie.

Cette incitation à l'immigration se traduit par une croissance rapide des effectifs de migrants en Libye, près de 100000 Soudanais et 40000 Subsahariens au recensement de 1995 contre 5000 et quelques centaines vingt ans auparavant. Bien que spectaculaire, cette croissance est de toute évidence sous-estimée. Des estimations récentes évaluent le nombre de ressortissants d'Afrique occidentale et centrale à environ 1,5 millions (The Baltimore Sun, 26.10.2000), dont 500000 Tchadiens (AFP, 1.10.2000) et un nombre supérieur de Soudanais (PANA, 8.03.2000). Pourquoi un tel décalage ? Sans doute parce que « l'étendue des frontières en Libye facilite l'entrée illégale de nombreux Africains » (Weekly Trust, 5.11.00) mais aussi parce que cette main-d'œuvre pléthorique, prête à accepter tous les types d'emplois pour de très faibles salaires, trouve désormais à s'employer dans le secteur privé, en pleine expansion.

La massivité des flux a rapidement activé une organisation transfrontalière de transit des migrants. De part et d'autre des frontières, des réseaux de transport fonctionnent, en taxis collectifs sur les courts trajets ou en camion sur les trajets plus longs. En général, les itinéraires des migrants africains convergent vers Agadez et de là ils optent pour l'Algérie, jusqu'à Tamanghasset où ils rejoignent les flux plus modestes depuis le Mali avant de se diriger vers le Maroc via Ghardaïa ou la Libye via Djanet, Ghat et Oubari. Mais la plupart des migrants effectuent le long trajet à travers l'Erg du Ténéré, empruntant les camions qui d'Agadez les conduisent à Dirkou, dans le Kawar, à proximité de la frontière tchadienne puis en Libye, à Al Qatroun et Sebha. Les Touaregs nigériens, habitués à emprunter ces axes, choisissent parfois de couper à travers le Ténéré entre l'Aïr nigérien et Djanet, selon le temps dont ils disposent, même si le chemin le plus court est souvent aussi le plus dangereux car il est soumis au brigandage et aux rackets. 
Une fois en Libye, si les nouveaux arrivants n'y disposent pas de coordonnées, ils se dirigent vers les diverses localités à la recherche de compatriotes. Ces derniers, souvent spécialisés par corps de métiers, facilitent ensuite leur insertion: les Nigérians travaillent généralement dans la mécanique, les Egyptiens dans l'agriculture, les Soudanais dans les petits commerces, les ressortissants du reste de l'Afrique de l'Ouest sont employés dans différents petits métiers ou dans la construction et ceux du Maghreb, du moins pour la dernière génération, dans le tourisme grâce à leur maîtrise du français.

Des réseaux économiques très actifs doublent les réseaux migratoires car la Libye est à bien des égards un « Etat-entrepôt » (L. Martinez, 1998) pour les Etats voisins. Cette situation s'explique par des décalages de niveau de vie, perceptibles avec le Sud tunisien et plus encore l'Egypte, flagrants vis-à-vis des Etats sahéliens, Niger, Tchad et Soudan. Ce facteur d'explication ne doit toutefois pas faire oublier l'importance des activités de négoce qui accompagnent les liens familiaux et tribaux transfrontaliers, inscrits dans la durée.

En apparence, le Sud libyen n'est pas la région centrale de ces échanges. Emmanuel Grégoire (1998) indique que «le Niger demeure... un carrefour d'échanges... entre l'Afrique noire et le Maghreb ». Dans le Tchad voisin, Karine Bennafla (1997) aboutit à des conclusions similaires, constatant que le pays est « écartelé entre le Nigéria, l'Arabie Saoudite, le Cameroun, la Libye et...le Soudan ». Toutefois, le carrefour géographique ne tient souvent qu'aux opportunités laissées dans des Etats qui n'ont pas d'emprise sur des régions entières du territoire national. Parfois, les responsables politiques y participent plus ou moins activement et ouvertement. Ainsi, au Niger, «le négoce arabe contrôle le gros commerce » (Grégoire, 1999). Selon les estimations de l'auteur, l'essentiel des échanges se concentre sur l'axe Tripoli-Sebha-Agadez; Sebha-Faya et Al Jawf-Faya sont secondaires. Le Sud-Est libyen est plus nettement orienté vers le Soudan.

Sur tous ces itinéraires, les flux sont à double sens. Du Nord vers le Sud, les produits subventionnés dominent les échanges. En effet, la nationalisation du commerce et la politique de redistribution en vigueur en Libye avaient contribué à ce que les produits, majoritairement importés, soient ensuite écoulés à travers le réseau des souks publics. Leur exportation depuis la Libye, devenue alors illicite, n'en a pas moins pris une vaste ampleur, comme l'atteste la diffusion des souks libya dans les villes marchandes des Etats voisins, d'El Oued en Algérie à Faya au Tchad 
et Khartoum au Soudan. De nombreux produits quittent ainsi la Libye, principalement ceux qui se conservent et supportent les rigueurs du voyage tels l'huile, les pâtes, le riz, le concentré de tomates mais aussi l'électro-ménager ${ }^{16}$ et les pièces détachées mécaniques mais aussi de nombreux biens de consommation. C'est donc une véritable économie du transit qui s'est développée autour des gares, d'où les camions partent à Sebha. Des marchés se développent où les migrants achètent les produits mais aussi les récipients et les lanières afin de les transporter, des garagistes réparent les camions, des cafés et des restaurants accueillent ceux qui sont sur le départ.

Ces échanges sont toutefois modestes au regard de ceux qu'organisent les grands commerçants. Ils concernent évidemment les produits subventionnés mais aussi les cigarettes ou le bétail. Les troupeaux de dromadaires et d'ovins transitent par Sebha et Al Jawf après avoir traversé le Sahara à pied ou en camions depuis le Niger et le Tchad. Ils sont vendus à un très bon prix, particulièrement durant l'Aïd al Kabir afin d'alimenter l'ensemble du marché libyen. Emmanuel Grégoire (1996 et 1999) décrit aussi un de trafic de cigarettes qui part du port de Cotonou au Bénin via Niamey, Agadez et Sebha jusqu'à Tripoli et Benghazi et se chiffre en milliards de francs CFA. Autre négoce, celui des devises, fondé sur la non-convertibilité du dinar libyen qui conduit à l'organisation d'un trafic illicite en Libye mais toléré voire reconnu dans les Etats voisins.

Ces échanges, qui relèvent pour une large part de l'informel, sont évidemment difficiles à chiffrer. Mais d'évidence, ils mettent en jeu des sommes considérables et mobilisent de multiples acteurs à différents niveaux hiérarchiques, du simple migrant aux grands commerçants. Loin de briser les solidarités sahariennes, les évolutions géopolitiques récentes se sont accompagnées de l'adaptation de la circulation des personnes et des produits à des contextes changeants.

\section{LES ÉCHELLES DU NOUVEAU CARREFOUR}

La fonction de carrefour s'appréhende donc à différentes échelles, celle de l'Etat libyen dans son entier qui, comme d'autres pays pétroliers, attire une main-d'œuvre importante, celle des agglomérations par lesquelles transitent hommes et produits. Tripoli est bien souvent le terminus de ces réseaux. Les agences de voyage, les grands parkings où sont garés bus ou semi-remorques à destination du Soudan, du Tchad ou du Niger

\footnotetext{
${ }^{16}$ La majeure partie des produits est fabriquée en Libye sous la marque Garyounis.
} 
témoignent de l'importance des échanges. Sebha, principale agglomération du Fezzan est un relais essentiel où se rejoignent les routes depuis l'Algérie, le Mali et le Niger. En fait, trois systèmes de circulation interdépendants s'emboîtent par le jeu des échelles. Chacun s'appuie sur un réseau urbain de plus en plus hiérarchisé au fur et à mesure que le cadre spatial s'élargit.

A l'échelle locale, le dynamisme récent des agglomérations des marges du territoire libyen doit beaucoup à l'intensification de la circulation transsaharienne comme l'illustre l'exemple d'Al Qatroun :

\begin{abstract}
La population d'Al Qatroun, sur la route du Tchad, enregistre un rythme moyen de croissance de $4.9 \%$ l'an. Le constat en 1944 était pourtant lapidaire: «voie de passage obligée des caravanes et des populations qui viennent du Soudan oriental et du Tchad ... ce tronçon de voie saharienne est presque devenu un cul-de-sac » (J. Despois, 1946). Ainsi, même le nombre important de jardins et de palmiers ne suffisait pas à enrayer le déclin de la région. L'arrêt de la circulation entraîna un déclin économique et démographique majeur. La reprise de la croissance provient de l'effet inverse, c'est-à-dire de la réactivation de l'axe de circulation vers le Niger. Depuis 1984, la croissance touche l'ensemble des villages de la région. Tajarhi, relais secondaire plus méridional connaît désormais la plus forte croissance, à l'intersection de la terminaison de l'axe goudronné libyen et de la piste qui le prolonge vers le Niger.
\end{abstract}

En effet, si les grands centres captent en priorité les flux de marchandises et de migrants, la diffusion progressive de l'immigration à l'ensemble des lieux habités du territoire est indéniable. Dans le Fezzan, le nombre d'étrangers résidents recensés représentait environ $4 \%$ de la population en 1973,9\% en 1984 et $8 \%$ en 1995, soit plus d'un doublement en valeur relative en 10 ans puis un tassement relatif entre les deux dernières périodes intercensitaires. Ainsi, alors que les effectifs d'étrangers triplent presque entre 1973 et 1995, passant de 103000 à près de 270000 , leur taux de croissance est officiellement moindre durant ces vingt années que celui de la population libyenne mais on sait désormais les limites de ces statistiques. 
Ces chiffres permettent néanmoins de pointer le fait que la diffusion de la présence immigrée dans le Fezzan s'effectue parallèlement à la croissance urbaine. En 1973, la majorité des agglomérations sont de petite taille et comptent peu d'étrangers, moins de $5 \%$ en moyenne. Mais les centres régionaux, tel Sebha, ou locaux, tels Ghat et Oubari attirent les migrants en priorité car ils y trouvent un emploi et des compatriotes plus aisément. Entre 1973 et 1995, la diffusion spatiale de la population étrangère s'accroît et si neuf agglomérations seulement en 1973 comptaient plus de $10 \%$ d'immigrés, elles sont dix-sept dans ce cas en 1984 et quinze en 1995.

Plusieurs facteurs permettent de comprendre cette diffusion spatiale de l'immigration. La croissance numérique du nombre d'étrangers en 10 ans est un premier facteur. La demande constante en main-d'œuvre, en est un autre, qui s'est maintenu durant chaque étape des changements socio-économiques du Fezzan, sous initiative étatique jusqu'au années 1980 et sous initiative privée depuis. En outre, les plus forts taux de population étrangère recensée se localisent en 1984 dans les petites villes situées le long de l'axe depuis l'Algérie d'Al Barkat à Oubari, celles où le peuplement touareg domine. Alors qu'en 1995 ces forts taux touchent les centres de la dépression de Mourzouq, à peuplement toubou, sur l'axe d'Agadès (Niger) et de Bardaï (Tchad) vers Sebha. L'installation des migrants dépend donc plus de la localisation des agglomérations des agglomérations sur les itinéraires empruntés par les migrants que de leur taille. Et le fait qu'il s'agisse de résidents recensés laisse penser que la plupart d'entre eux, installés en Libye après avoir quitté définitivement leur pays d'origine, y obtiennent ensuite la nationalité libyenne.

Les migrations internes ont été l'un des moteurs initiaux du processus d'urbanisation. Les principaux pôles offraient de plus grandes opportunités d'emploi et ont capté les flux en priorité. Les migrants internationaux, en revanche, paraissent s'inscrire dans la trame urbaine en place, sans réellement en modifier l'architecture. La route reste le plus sûr vecteur de leur répartition sur des itinéraires plus vastes.

A l'échelle régionale, Sebha et $\mathrm{Al}$ Jawf, côté libyen, sont reliées à Tamanghasset, Agadez, Faya, ensembles de nœuds spatiaux d'un espace migratoire de proximité. Ces nouveaux carrefours sont généralement les villes que les autorités nationales ont choisies comme centres administratifs régionaux. Elles remplacent dans ces fonctions les vieux centres du commerce transsaharien tels Ghat et Mourzouq dans le Fezzan. C'est de là que partent les camions chargés de biens de consommation, de produits de contrebande, de bétail et de migrants qui circulent d'une région 
à l'autre. C'est aussi là que se situent les acteurs de ces circulations, fonctionnaires ou commerçants.

Figure 8 : Le carrefour migratoire libyen

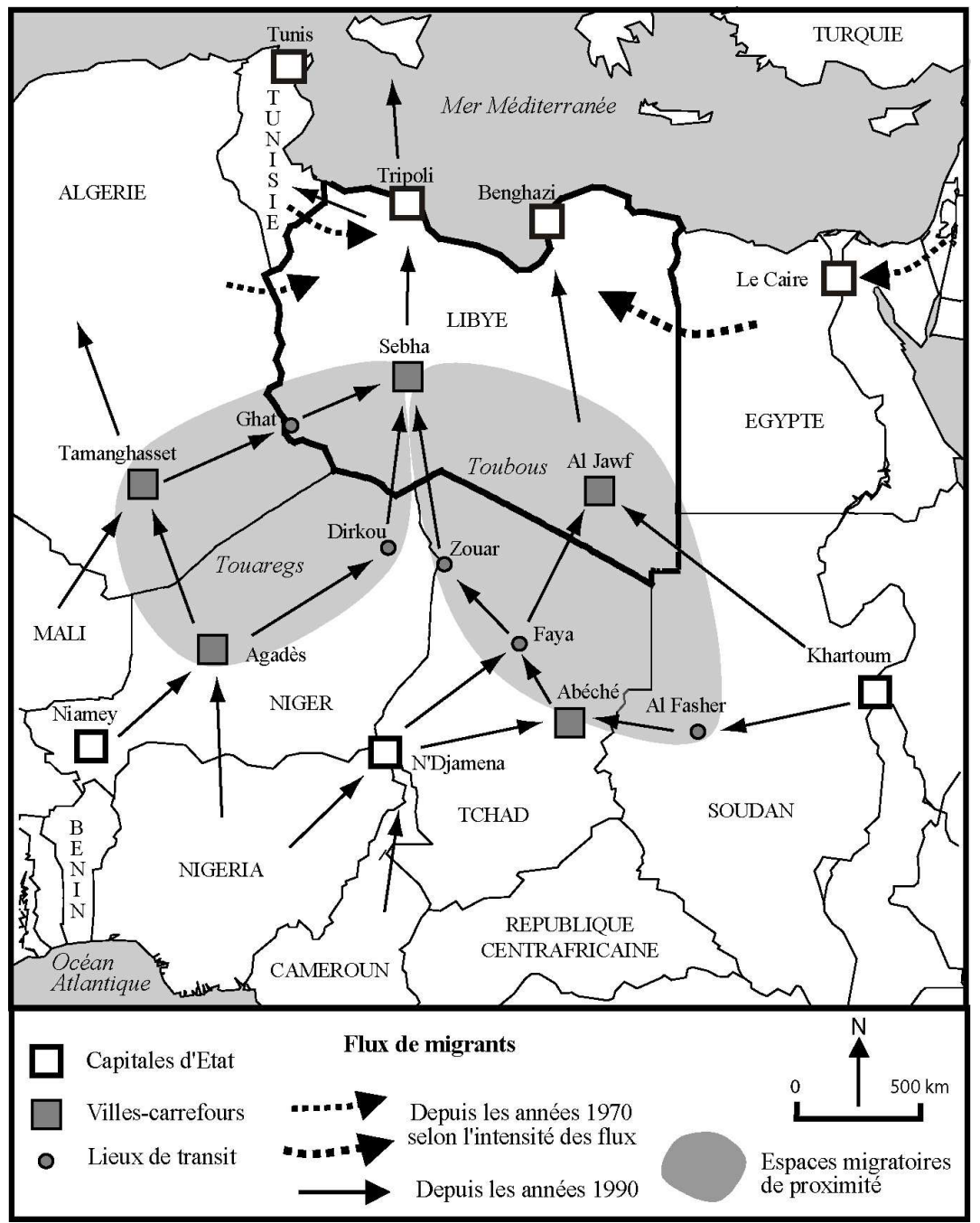

Al Jawf, dans le groupe d'oasis de Koufra, le plus isolé $\mathrm{du}$ territoire libyen et le plus proche des frontières soudanaise et tchadienne n'était qu'une bourgade de 2 000 habitants en 1954. La population croît ensuite rapidement puisqu'elle double durant chacune des deux 
décennies suivantes. Depuis les années 1980 , le taux de croissance se maintient à près de $6 \%$ par an. Aujourd'hui, Al Jawf compte près de 30000 habitants, conséquence d'une croissance qui repose sur la volonté étatique de construire un pôle administratif et de développer l'un des plus ambitieux projets agricoles d'irrigation saharien. Al Jawf, seconde ville du Sahara libyen après Sebha, cumule les mêmes fonctions de centre administratif et de carrefour vers le Soudan et le Tchad. D'ici partent ou transitent vers le littoral des camions chargés de personnes et de produits depuis Abéché et Khartoum, faisant de la ville une plaque tournante commerciale de première importance. Si l'agglomération actuelle a été façonnée autour d'un des projets agricoles les plus médiatisés, puis a bénéficié de sa fonction de base-arrière durant la guerre entre la Libye et le Tchad, c'est à nouveau la fonction de transit qui assure à Al Jawf son dynamisme actuel. Alors que le désengagement étatique se fait ressentir ici avec plus de force que dans le Fezzan, l'économie de transit offre des opportunités lucratives pour les Libyens de cette région isolée.

L'échelle régionale permet de prendre la mesure de la perpétuation du réseau transsaharien dans la continuité historique de celui qui avait périclité au tournant $\mathrm{du} \mathrm{XX}^{\text {ème }}$ siècle. La continuité dans le principe n'empêche pourtant pas une rupture dans les modalités de l'organisation spatiale. Désormais, les principales agglomérations se situent dans le Sahara central et l'on ne relève pas la présence de relais entre celles-ci et le littoral. La question de la distance ne se pose pas non plus avec la même acuité qu'à l'époque du commerce caravanier.

A l'échelle du fuseau Libye-Afrique, cet espace de circulation régional a des connections hors du Sahara vers Niamey au Niger, N'Djamena au Tchad et Khartoum au Soudan. Ce sont évidemment des capitales mais aussi des villes reliées à Tripoli par la voie aérienne via Sebha. Par ailleurs, au-delà des Etats sahéliens proches, les métropoles du littoral ouest-africain sont de plus en plus nettement intégrées à ces réseaux car des produits de contrebande y sont déchargés dans les ports et de nombreux travailleurs viennent de là après y avoir obtenu des renseignements sur les filières permettant de se rendre en Libye. 
La distinction de ces trois échelles d'analyse permet donc de souligner les intrications croissantes entre trois types de circulations. Les agglomérations sont des lieux majeurs sur les itinéraires transsahariens, car, l'urbanisation progressant, c'est de plus en plus de ville à ville que l'on circule. La circulation contribue notamment à créer de nouvelles centralités spatiales qui complètent -ou s'effectuent en marge de-celles que l'intégration au territoire national avait produit, et ceci à toutes les échelles, des centres ruraux où se fixent les nomades, aux centres régionaux dans le cas de l'organisation des échanges et des déplacements transfrontaliers, parfois même dans les capitales. La contribution à cette centralité s'atténue évidemment au fur et à mesure que l'on monte dans la hiérarchie urbaine mais dans les limites du Sahara, son rôle est déterminant. En conséquence, les espaces urbains se transforment en proportion de la taille et de la centralité des agglomérations concernées, dans leurs fonctions mais aussi dans les manières d'habiter des villes du Fezzan. 


\section{LES CYCLES URBAINS DU FEZZAN}

Dans le Fezzan, quatre temps forts rythment donc l'articulation entre logiques d'organisation de l'espace et dynamiques urbaines durant le $\mathrm{XX}^{\text {ème }}$ siècle.

Le premier cycle de l'organisation spatiale se fonde sur deux éléments essentiels, l'échange et la distance. L'échange entraîne l'émergence de villes sahariennes (Mourzouq, Ghadamès), véritables carrefours situés à la confluence des axes de l'échange. Entre ces centres, les relais sont nombreux, puits, villages isolés, ensembles oasiens. En les recensant, on retrouve le fondement de la trame de peuplement actuelle. Au bout des axes, des ports, ouverts vers l'extérieur rappellent le primat du réseau sur le territoire. Les limites, plus que les frontières entre aires d'influence des puissances dominantes situées hors du Sahara, transforment souvent les régions-carrefours du désert en hinterland. Selon les vicissitudes géopolitiques du moment, des villes déclinent, d'autres émergent, les axes se déplacent mais conservent leur organisation méridienne.

Du début du XX ${ }^{\text {ème }}$ siècle aux années 1970, le Fezzan est un territoire sans villes parce que la domination coloniale, brève, brutale et partielle achève la déstructuration de l'organisation spatiale fondée sur le commerce. Le contrôle devient l'élément majeur de la structuration spatiale avec la multiplication des forts, des centres administratifs et des aéroports souvent distincts des vieux foyers de peuplement oasiens. Mais au-delà des apparences de la décadence la trame sur laquelle s'appuie ensuite l'Etat issu des indépendances se dessine.

Le troisième cycle, celui de l'intégration nationale, se caractérise d'abord par la fermeture du Sahara libyen, devenu partie d'un territoire d'Etat. En l'espace d'une trentaine d'années, les mutations sont spectaculaires: le réseau routier est étendu, la vieille agriculture de palmeraie est rénovée, les populations nomades se fixent et tous bénéficient des subsides d'un Etat redistributeur. L'uniformité spatiale est à l'ordre du jour; la multiplication des agglomérations, considérées comme des lieux de concentration des personnes, s'accompagne de l'imposition d'un modèle-Etat d'urbanisation. C'est par le haut que la dynamique d'urbanisation-modernisation des vallées oasiennes s'effectue. En parallèle, la coupure Nord-Sud avec les comptoirs sahariens situés dans les Etats du Sahel s'accroît.

Actuellement, la moindre intervention de l'Etat, une porosité croissante des frontières entre Etats et la réactivation des réseaux sahariens se 
conjuguent pour dessiner un nouveau contexte. Il serait sans doute erroné de conclure à la substitution d'une dynamique du réseau à celle de la logique stato-nationale. Les échanges ne contribuent en effet pas à la restructuration globale de l'organisation spatiale mais ils s'immiscent dans les interstices du territoire national. A l'échelle locale, le dynamisme des agglomérations chefs-lieux, des plus petites à Sebha, et parfois même au-delà des frontières, s'explique en partie par les flux qui y transitent, les personnes qui y passent ou s'y installent. On ne peut penser le réseau régional et les évolutions de l'urbain sans tenir compte de cette dimension saharienne. En somme, des indices probants émergent de la conjonction de deux dynamiques souvent opposées, le temps long du réseau et le temps court du territoire d'Etat. 


\section{DEUXIÈME PARTIE}

\section{DES DYNAMIQUES LOCALES ENCADRÉES PAR L'ETAT}

L'Etat libyen a tenu une place déterminante dans le déclenchement du processus d'urbanisation du Fezzan, l'inscrivant dans un projet de plus vaste ampleur, celui du façonnement d'un territoire et d'une société. Mais que devient ce projet à l'épreuve du désengagement étatique ?

Les autorités publiques n'agissent plus désormais de manière unilatérale comme cela était le cas durant les trois décennies précédentes. Les mesures de libéralisation prises depuis la fin des années 1980 se sont accompagnées d'une redéfinition des cadres de l'action étatique au niveau local et de l'émergence d'acteurs dans tous les domaines d'activités, économiques, politiques et sociaux. Si la carence en informations rend aléatoire toute généralisation, il apparaît néanmoins que l'Infitah (l'ouverture, la libéralisation) a incontestablement contribué à l'émergence d'un cadre local dans lequel se déploient de nouveaux champs d'action.

Les agglomérations n'évoluent donc plus seulement désormais sous l'impulsion des initiatives étatiques mais aussi sous l'effet des actifs changements sociaux qui traversent la société fezzanaise aujourd'hui. Le modèle étatique d'urbanisation se délite et l'espace urbanisé porte la marque des différenciations sociales au fur et à mesure de son extension, celles que le pouvoir central avait tenté de gommer auparavant.

L'espace rural ne subit pas la désaffection que pourrait laisser supposer un processus d'urbanisation intense. S'il était encore besoin de le prouver, la croissance des villes ne se fait pas non plus au Sahara au détriment des campagnes. Ces dernières évoluent au contraire sous l'influence directe de l'urbain par une conjonction de facteurs que l'Etat s'est partout employé à combiner : regroupement des populations, création ou modernisation de périmètres irrigués, désenclavement routier, dotation des centres urbains en fonctions élémentaires d'éducation et de santé notamment. Durant les années 1990, l'émergence d'acteurs privés dans un cadre familial entraîne de nouvelles transformations, tant économiques que paysagères et sociales.

On aura compris que si le premier niveau de lecture vise au décryptage des dynamiques spatiales, la manière dont les sociétés agissent sur cet 
espace constitue un fil directeur d'analyse. Le glissement d'une initiative étatique à une initiative privée se manifeste partout avec force. Pour autant, les monographies et le repérage des principales mutations spatiales laissent entrevoir des dynamiques locales étroitement cantonnées dans un cadre fixé par le haut. 
CHAPITRE 4 - TRANSACTIONS ENTRE LE NATIONAL ET LE LOCAL

La rapidité du processus d'urbanisation suscite-t-elle ou correspond-elle à l'émergence de nouveaux acteurs dans le Fezzan ? Et dans ce cas, quelle est leur marge de manœuvre?

Il est nécessaire de sortir du cadre saharien pour aborder ces questions notamment pour comprendre quelques-unes des logiques de l'«Etat sans Etat », parfois perçu comme décentralisateur mais en définitive fondamentalement jacobin dans ses pratiques (F. Burgat, A. Laronde, 1996). Malgré la carence en études de cas récentes, quelques traits majeurs se dégagent, qui révèlent un «cas libyen» aux rouages politiques, économiques et sociaux souvent peu différents de bien d'autres pays arabes. C'est sans doute en partie parce que les bouleversements sociaux, notamment l'urbanisation généralisée et la croissance démographique, perturbent le fonctionnement politique jusqu'alors circonscrit aux relations entre l' «Etat et les tribus » (J. Davis, 1987). Avec réticence, les autorités libyennes poussent plus avant l'Infitah économique, annoncée en 1987 puis freinée durant la période d'embargo mais que les acteurs de l'activité économique privée réclament avec force.

Dans le Fezzan, deux éléments caractérisent le contexte politique, économique et social actuel: le cadre d'action est avant tout local et familial. Pour autant, ce cadre n'est pas statique, faisant preuve d'une adaptation remarquable aux changements qui touchent la région. Nous énumèrerons quelques entrées afin d'en cerner les contours : la croissance des activités informelles, celle du nombre de migrants, les extensions urbaines ou les investissements dans les activités agricoles. L'exemple de Ghadamès est d'autant plus intéressant qu'il rompt avec la tendance générale et rappelle à quel point le rôle des acteurs locaux est d'autant plus notable qu'il s'inscrit dans la durée.

\section{L'OMNIPRÉSENCE DE L'ETAT SANS ETAT}

Le système politique libyen des années 1970 présente des particularités qui conduisent l'observateur à le distinguer de ceux des autres Etats du Maghreb. Trois grilles de lecture paraissent fondamentales afin de comprendre la Libye contemporaine (R. Bocco, 1988). 
La principale particularité est, d'évidence, l'importance de la rente pétrolière ${ }^{17}$ qui a permis aux dirigeants libyens de disposer de ressources importantes sans avoir à taxer leurs concitoyens et de prendre en charge ces derniers en les employant massivement dans la fonction publique. Cette opportunité a eu des avantages immédiats, notamment celui de laisser les mains libres aux dirigeants exempts de l'obligation de rendre compte de leurs choix politiques, exempts aussi de toute velléité de perfectionnement de l'appareil d'Etat. Mais les limites de ce mode de fonctionnement sont toutes aussi flagrantes. L'économie rentière libyenne se caractérise par une triple dépendance, vis-à-vis de la variabilité du prix du baril de pétrole, de l'importation massive de biens de consommation et de technologie et de besoins massifs de main-d'œuvre étrangère. Toute crise économique est donc de nature à fragiliser la Libye et par-là les fondements de la légitimité de ceux qui la gouvernent. Or, dès le début des années 1980, la chute des cours des hydrocarbures met progressivement à mal le système économique puis politique libyen, et par conséquent les modalités selon lesquelles la rente était redistribuée à la population. On peut lire dans les changements que la Libye a connus durant les années 1990 la manière dont les autorités ont tenté d'adapter l'appareil d'Etat à ce nouveau contexte.

La seconde particularité du régime politique libyen réside dans la volonté des autorités, en l'occurrence du Guide et théoricien de la Révolution, Mouammar Kadhafi, de médiatiser l'image d'absence d'Etat.

Plusieurs raisons ont amené le pouvoir central à ce choix : le fait que la Libye n'a pas hérité d'un modèle étatique colonial, cette période ayant été courte, brutale et dénuée de toute velléité d'émergence d'élites locales qui auraient géré la succession italienne; la vision suspicieuse que porte Kadhafi envers les institutions étatiques et donc au refus non seulement de l'Etat mais aussi de toute forme de représentation. La Jamahiriya (Etat des masses) se définit par l'abolition de tout gouvernement central et de remise du pouvoir aux unités de base au sein desquelles la population est regroupée (R. Bocco, 1988). Parler d'Etat en Libye revient donc à employer un terme générique, une commodité de langage plus qu'une réalité clairement définie. Il est dès lors nécessaire de se questionner sur le «système Kadhafi » afin de mieux comprendre le fonctionnement institutionnel libyen. Le schéma institutionnel présente théoriquement une

\footnotetext{
${ }^{17}$ En 2000 , le pétrole brut et les produits dérivés composent environ $95 \%$ des exportations annuelles libyennes. Les réserves prouvées de brut en Libye, quoique très inférieures à celles de l'Arabie Saoudite (263,5 milliards de barils), sont les plus importantes d'Afrique. En 2000, avec 29,5 milliards de barils de réserves, la Libye se place devant le Nigeria (22,5 milliards de barils) et devant l'Algérie (9,2 milliards de barils).
} 
grande souplesse. Le pouvoir juridique appartient aux congrès populaires et le pouvoir exécutif aux comités populaires. Ils ont « une base territoriale (quartiers et municipalités) ou sectorielle (par activités)» (F. Burgat, A. Laronde, 1996). La réunion annuelle des congrès, en un Congrès Populaire Général, vise à avaliser et à harmoniser les décisions prises par chacun d'eux et à les doter ou non d'un financement. Le comité populaire général, instance exécutive, correspond à un gouvernement dont les secrétariats sont les ministères.

Les relations entre les niveaux de pouvoir local et national s'effectuent sans échelons intermédiaires, de niveau régional, aux attributions clairement identifiées mais de nombreux paramètres viennent perturber ce mode de fonctionnement. Indéniablement le fait que «l'autoritarisme jacobin » prédomine limite d'autant les marges de manœuvre du pouvoir local. De plus, «dans la pratique, l'autonomie de décision des congrès de base va s'avérer assez largement fictive (car) les appartenances traditionnelles et leurs logiques mobilisatrices propres se sont très vite coulées dans les failles de ce système pour le détourner » (Ibid, 1996).

L'assise tribale de la société joue donc un rôle essentiel qui conduit à considérer la relation entre "l'Etat et des tribus » comme fondatrice de l'action politique dans la Jamahiriya. C'est là la troisième particularité du système politique libyen.

Les recherches de John Davis (1987), qui analyse les relations de pouvoir au niveau local et leurs articulations avec le système politique national libyen, sont un point de départ incontournable. L'auteur montre notamment comment «l'organisation tribale... se reproduit dans la Jamahiriya (à une époque où) l'arène politique du pays est relativement petite » (R. Bocco, 1988). Quelques clés d'explication sur les modalités de l'agir en Libye découlent du rapport entre l'Etat et les tribus notamment lorsque «de ce principe de fonctionnement qui caractérise la société, découle la physionomie actuelle des villes libyennes, leur peuplement, leur bâti... (Elles sont), en définitive la négation même du creuset urbain » (J. Bisson, 1997).

Lorsque John Davis mène ses études de terrain entre 1975 et 1979, dans la partie orientale du pays, les trois entités régionales de Tripolitaine, Cyrénaïque et Fezzan étaient alors loin d'être homogènes, présentant des différences notables, selon leur inégale affiliation à la Senoussiya, puis leurs rapports aux régimes successifs : la Cyrénaïque, à l'est, était un soutien à la Royauté et le foyer de la dissidence envers le régime de Kadhafi alors que la Tripolitaine et le Fezzan, à l'ouest avaient des positions inverses. Depuis, deux problèmes se posent, celui de savoir si ces 
informations valent pour toute la Libye et si elles permettent de comprendre la période actuelle car on admettra aisément qu'entre-temps le contexte politique, la société et les modalités d'exercice du pouvoir aient pu largement évoluer. Si les analyses de John Davis permettent de comprendre les modalités de la production de l'urbain durant la période d'intervention étatique, sont-elles aussi pertinentes pour la période actuelle?

L'évolution des découpages administratifs est sans doute symptomatique de la délicate répartition des pouvoirs entre les différents niveaux d'encadrement du territoire, national, régional et local. En Libye, le système initialement décentralisé semblait susceptible de favoriser, bien avant les Etats voisins, l'émergence d'un pouvoir local institutionnalisé. L'instauration de la Jamahiriya, en 1977, se traduit par une inflation du nombre de municipalités et d'annexes, émanation de la vision «kadhafienne» d'une large décentralisation des pouvoirs. Mais si le Fezzan est divisé en cinq municipalités et trente-neuf annexes en 1978, les découpages suivants consistent en une rétraction du nombre d'entités administratives particulièrement drastique: trois municipalités et six annexes en 1986 puis une et quatre en 1991. Le mouvement est contraire à celui que l'on constate au même moment en Tunisie ou en Algérie puisqu'en Libye on passe progressivement d'un maillage local à un maillage «micro-régional ». Le découpage de 1997 renoue avec celui de 1978, proposant un morcellement identique. L'interprétation de l'évolution du maillage administratif libyen est donc pour le moins délicate ; elle ne traduit ni n'accompagne la croissance et l'agglomération de la population, contrairement à la tendance observée dans le reste du Sahara maghrébin. Le découpage de 1978 traduit en fait une vision idéologique du territoire, très décentralisée au plan local, alors que les découpages suivants correspondent aux aires d'action d'aménagement du territoire.

Le dernier découpage marque en apparence un retour vers la tendance initiale, amplifiée même, puisque le nombre d'annexes double. Pourtant, certains changements de dénomination ne trompent pas. Ainsi les centres (marâkaz) sont désormais des villes (moudoun), telles Oubari, Mourzouq et Ghat. De plus, la majorité des découpages correspondent à des ensembles villageois cohérents. De toute évidence, ce découpage prend acte de l'urbanisation ou plus précisément d'une micro-urbanisation linéaire ; elle se caractérise par la multiplication des pôles qui forment la trame d'un réseau de villages dont nous avons rappelé l'ancienneté. L'évolution sémantique consacre donc l'émergence d'une trame urbaine hiérarchisée et structurée par les principales agglomérations. Les choix 
libyens de maillage territorial semblent progressivement accompagner la mutation spatiale du Sahara libyen mais ils nous disent sans doute plus sur les délicates relations entre le national et le local. C'est probablement en marge de cette organisation que l'on peut réellement lire l'émergence d'acteurs du local.

La récente décision de décentraliser les pouvoirs va peut-être aussi dans le sens d'une plus grande lisibilité des échelons du pouvoir administratifs en créant en 1998 avec les Sha abiyat un niveau régional d'encadrement du territoire plus affirmé. Il semble qu'après plusieurs scenarii, il ait été décidé de stabiliser le découpage à 26 unités, dont quatre pour le Fezzan : Wadi Ash Shati, Sebha, Wadi Al Haya et Mourzouq ; on retrouve ainsi les quatre grands ensembles qui composent la région, l'ensemble oasien de Ghat étant rattaché au Wadi Al Haya. Cette réforme frappe par son ampleur, 21.5\% du budget national est alloué aux Sha`abiyat en 1998, 38 $\%$ en 1999 et $48 \%$ en 2000. La dissolution de l'appareil d'Etat, longtemps prônée, se concrétise alors que le discours dogmatique sur l'Etat sans Etat a désormais perdu l'essentiel de sa substance, conférant à l'échelon régional une capacité d'intervention inédite dans l'histoire de la Libye contemporaine. Le Fezzan ne paraît pas particulièrement avantagé par cette réforme, bénéficiant d'une dotation budgétaire sensiblement équivalente à son poids démographique à l'échelle nationale. En revanche, il paraît opportun de se questionner sur ceux qui bénéficient d'une décentralisation aussi massive. A bien des égards, la déconcentration des moyens financiers dont dispose l'Etat libyen paraît profiter aux élites locales proches du régime, renforçant ainsi de nouvelles formes de clientélisme, plus discrètes.

Ainsi, par-delà les allers-retours entre différentes formes d'encadrement $\mathrm{du}$ territoire national se devinent les indispensables transactions auxquelles doit procéder l'Etat libyen face aux structures sociales locales.

\section{DE L'INFITAH (OUVERTURE) À LA MUTATION DE L'ÉCONOMIE LOCALE}

Partir du principe que le désengagement de l'Etat aurait suscité l'émergence d'acteurs n'est sans doute pas erroné mais le raccourci est pour le moins réducteur. Certes, l'année 1987 est marquée par « le virage de l'Infitah économique et de la perestroïka politique » (F. Burgat, A. Laronde, 1996). Mais Dirk Vandewalle (1995, 1998) a démontré les limites du mouvement, soulignant qu'à la place de « l'Infitah nationale », les gouvernants ont privilégié une modeste « Infitah de la consommation». 
Comment et pourquoi, se demande-t-il, après une intense période d'étatisme, le gouvernement semble-t-il incapable de libéraliser l'économie?

Dirk Vandewalle explique ces paradoxes par l'incapacité de la Libye à rompre avec le fonctionnement d'un Etat rentier: une incapacité qui s'inscrit selon lui dans la volonté du pouvoir de maintenir des rapports clientélistes avec les soutiens au régime, et plus largement avec l'ensemble de la population, élément indispensable au maintien de sa légitimité. En conséquence, la situation actuelle se caractérise par de nombreux paradoxes. Les produits de consommation de base sont toujours distribués à bas prix par le biais des souks d'Etat mais en parallèle, les commerces privés, autorisés à rouvrir, vendent les produits que ne fournit pas le circuit officiel, ceux qui correspondent à une demande croissante dans une société en plein changement. Les commerçants s'approvisionnent par le biais d'un marché parallèle toléré de biens de consommation en provenance de Malte, Istanbul et Dubaï.

Depuis une décennie, les mutations de l'économie locale du Fezzan sont rythmées par les étapes de la libéralisation et du désengagement de l'Etat. Les contours de cette économique révèlent les carences qui minent de plus en plus nettement le système de redistribution de la rente et reflètent les disparités croissantes qui divisent les populations du Fezzan, entre Libyens et non-libyens, possédant ou non, propriétaires et locataires, comme le reflètent les exemples suivants.

La croissance des activités informelles constitue l'élément le plus voyant de la mutation de l'économie locale. Elle renvoie à une évolution générale que l'on retrouve sous des formes similaires dans l'ensemble du Monde arabe (R. Escallier, 1998). Dans le Fezzan, l'économie informelle est largement présente du fait de la double activité qui touche toutes les couches sociales. Les fonctionnaires et les étudiants forment les plus importants contingents de gérants et de propriétaires des échoppes qui se sont multipliées dans le tissu urbain de tous les villages et les villes.

L'essor du secteur touristique permet de pointer des formes d'entreprenariat de plus grande ampleur, dépassant des stratégies individuelles et structurées à l'échelle de l'ensemble de la région. La libéralisation et la croissance rapide du nombre de visiteurs ont entraîné la multiplication des petites agences de voyages, à l'initiative de particuliers, souvent Targui, qui proposent leurs services en tant que guides. Il y aurait à Ghat plus d'une vingtaine d'agences de ce type mais les plus importantes se situent à Sebha ou à Tripoli. Leurs propriétaires sont souvent natifs du Fezzan mais leurs moyens financiers et leurs qualifications leur permettent 
d'organiser des filières reliées aux foyers émetteurs. En conséquence, ils intègrent les petites agences locales dans des réseaux coordonnés à partir de Sebha, subordonnants les moins nantis des acteurs du secteur touristique aux agences les plus importantes et construisent des infrastructures touristiques sur les lieux les plus visités.

La place des travailleurs immigrés en Libye est au cœur des changements économiques actuels. $\mathrm{Si}$ cette main-d'œuvre était indispensable au fonctionnement de l'économie étatisée, son rôle parait être tout aussi déterminant au développement de l'économie privée. Il faut peut-être rappeler que dès le milieu des années 1970, un actif sur trois n'avait pas la nationalité libyenne et que durant les années 1980, les étrangers composaient plus de la moitié de la population active en Libye (F. Burgat, A. Laronde, 1996).

Actuellement, la majorité des jardins sont entretenus par des paysans égyptiens ou maghrébins qui travaillent pour le compte d'un propriétaire libyen. Mais l'articulation entre les activités liées à l'agriculture et l'emploi des migrants semble parfois prendre une toute autre ampleur. La volonté de rendre les exploitations plus productives incite les autorités à privilégier les acteurs les plus dynamiques de l'agriculture, en l'occurrence les Egyptiens. A Ad Disa, près d'Oubari, l'Etat leur loue directement des exploitations sur le nouveau périmètre agricole. Il fournit les intrants, ensuite le produit de la récolte est racheté à la moitié de sa valeur estimée, ou le locataire verse une contrepartie financière équivalente à ce montant. Cette solution donne aux Egyptiens, très bien insérés dans le système agricole libyen, la possibilité d'écouler eux-mêmes leur production. Ils s'adressent à leurs compatriotes qui ont mis en place des filières de vente orientées vers le marché de gros de Tripoli, sur la route d'Al Aziziya au sud de la ville. L'orientation commerciale détermine les types de cultures à forte valeur ajoutée (melons d'eau, agrumes, raisin) au détriment de celles qui sont plus répandues et donc exposées à des chutes de prix durant les périodes de surproduction, notamment les oignons. Cette organisation constitue une véritable nouveauté dans un système où les nationaux ont toujours été privilégiés. Les étrangers n'ont légalement pas accès à la propriété mais 
ils ont acquis un poids prépondérant dans certains secteurs, au point d'en devenir des acteurs essentiels.

De même, la présence croissante de subsahariens explique le développement de commerces et d'activités plus spécifiques à cette région, tel que l'artisanat des forgerons, en bord de route ou les multiples intermédiaires de l'économie de transit qui animent une large partie du commerce de Sebha. Le secteur touristique doit une partie de sa structuration rapide à l'importance des effectifs de migrants, tels les jeunes guides ou cuisiniers touaregs nigériens, mobilisés dans le cadre lignager, afin de travailler durant la saison touristique dans le Fezzan, ou les Maghrébins pour leur pratique de la langue française.

Les mutations économiques du Fezzan reflètent donc le changement social mais aussi les réseaux dans lesquels est insérée la région. Par exemple, la quasi-totalité des produits vendus dans les boutiques sont importés, parfois par le biais de la contrebande avec les pays frontaliers comme c'est le cas pour les multiples échoppes où l'on écoule des cigarettes de marque américaine fabriquées au Niger ou au Mali. Instrument de régulation sociale à l'intérieur, l'économie informelle devient en conséquence un facteur de relations avec l'extérieur dans un contexte de fermeture politique. La forme la plus classique, que l'on retrouve dans les pays étatisés, est celle des marchés noirs.

Mais c'est beaucoup plus profondément que se transforment les rouages de l'économie locale comme les recompositions de l'offre et de la demande dans le secteur de la construction le laissent apparaître.

Les évolutions du secteur du bâtiment sont symptomatiques des évolutions récentes de l'appareil gouvernemental. Les différentes branches de ce secteur du bâtiment sont réparties entre plusieurs comités populaires généraux : le comité de l'habitat et des services publics prend en charge l'habitat, en particulier les logements sociaux; les centres de santé et les hôpitaux relèvent du comité de la santé; le comité de l'agriculture gère les projets agricoles de peuplement ; le comité du tourisme doit prendre en charge la construction des infrastructures hôtelières et les aménagements liés à cette activité ; enfin, un centre de la planification urbaine est chargé de coordonner les différentes initiatives en vue d'un aménagement du territoire cohérent.

Ces actions sectorielles relèvent donc d'un nombre limité de comités populaires. Mais certains d'entre eux bénéficient de revenus (perception de taxes) et de dotations qui leur donnent la possibilité d'agir hors de leur 
champ de compétence établi. Ils créent des sociétés dépendantes qui ont en charge des projets précis de construction de logements, d'hôtels, de périmètres agricoles. En outre, les incitations au développement de l'initiative privée s'accompagnent d'une remise en cause du système qui prévalait jusque-là, celui des projets «clés en main » exécutés par des entreprises étrangères. Les sociétés locales se multiplient donc afin d'obtenir des contrats ou de servir d'intermédiaires. Elles sont généralement dirigées par des hauts fonctionnaires, des militaires, des personnalités proches du pouvoir.

En définitive, le nombre d'acteurs s'accroît et cette croissance s'accompagne d'intrications croissantes entre acteurs publics et privés. Les premiers acquièrent une visibilité institutionnelle alors que leurs relations avec les seconds deviennent plus difficiles à appréhender (P. Signoles, G. El Kadi, R. Sidi Boumedine, 1999).

La pénurie en logements est source de fortes distorsions entre l'offre et la demande. La demande se modifie sous l'effet du croît démographique des Libyens. La pénurie est donc essentiellement quantitative puisque la construction stagne alors que le nombre de jeunes en âge de s'installer a considérablement crû, conséquence d'un taux de natalité soutenu sur une longue période. Le problème du logement devient alors problème social puisqu'il faut économiser des sommes plus importantes pour avoir accès à la propriété... et donc pouvoir fonder sa propre famille. On mesure ce que cela peut entraîner comme frustrations et recherche de revenus en vue de constituer l'indispensable pécule. Dans ce cas, la stratégie est individuelle et a pour objet l'adaptation, difficile, des individus à de nouvelles contraintes afin de construire un projet familial surtout si sa famille ne dispose pas des moyens nécessaires afin d'aider ses membres. En conséquence, le nombre de Libyens qui résident dans de vieux villages ou qui se sont installés sur les ruines des ksour croît rapidement. Ils bénéficient généralement d'une propriété familiale, établie de longue date dans ce site. Plus problématique est le cas de ceux qui s'installent dans une agglomération différente de celle dont ils sont natifs, comme les étudiants. La pénurie d'habitat public touche en effet aussi les logements universitaires. S'ils veulent trouver un lieu de résidence et s'ils deviennent tributaires de l'offre disponible sur le marché locatif. Or, officiellement, la location d'un logement demeure illicite... On comprend alors pourquoi la forte croissance de l'habitat non-réglementaire joue pour beaucoup dans l'extension spatiale des agglomérations.

La question du locatif se pose avec plus d'acuité encore pour les étrangers. Au-delà de la durée de leur séjour, saisonnière pour les uns, 
presque définitive pour d'autres, ils se trouvent en effet confrontés à un paradoxe insoluble : celui de ne pouvoir être, légalement, ni propriétaires ni locataires. Ce paradoxe est contourné par la nécessité selon plusieurs modalités. On peut parler de tolérance lorsque les autorités locales décident de construire un immeuble pour loger les enseignants, majoritairement égyptiens ou irakiens, du village. Mais en général, c'est plutôt la tolérance qui est de mise, lorsque les étrangers s'installent dans les agglomérations, forcément de manière illégale... compte tenu des contraintes que la loi impose.

On devine, au vu de ces exemples, comment se met progressivement en place un marché de l'immobilier dans un pays où la construction et la distribution des logements relevaient jusqu'à présent exclusivement des pouvoirs publics. En se désengageant du secteur de la construction, l'Etat en délègue la gestion aux acteurs du local, permettant aux plus nantis d'envisager des placements spéculatifs. Ces derniers sont d'abord à l'initiative d'un important mouvement d'autoconstruction, logements ensuite loués à des Libyens ou, surtout, à des étrangers. De plus, ils peuvent s'installer dans des résidences plus luxueuses tout en louant leur logement individuel public pour un montant souvent conséquent. Les croissances parallèles de l'habitat résidentiel privé et du non-réglementaire expliquent pour une large part l'extension et la différenciation croissante des tissus urbains. Dans les faits, elles sont souvent reliées car les mêmes personnes sont à l'origine de l'un et de l'autre, agissant comme de véritables promoteurs dans les villages et les villes du Fezzan.

\section{DES ACTEURS ENTRE CADRE FAMILIAL ET ÉTATIQUE}

Comment se déploient les stratégies des individus dans le Fezzan contemporain ? Des monographies, itinéraires dans le réseau villageois du Wadi Al Haya, permettent de prendre la mesure du cadre qui se dessine aujourd'hui. Ce cadre présente des divergences fondamentales selon les niveaux de revenus et la place des personnes au sein des hiérarchies sociales mais aussi des convergences indéniables qu'il s'agisse des relations que chacun entretient avec son cadre familial ou vis-à-vis de l'action des pouvoirs publics.

Deux portraits d'exploitants agricoles permettent de comprendre comment se mettent en place de nouvelles formes de médiation, entre le cadre familial et l'intervention étatique. 
Brahim vit dans un nouveau village qui ne date que d'une dizaine d'années. Sa famille vivait jusque-là dans l'Erg d'Oubari, sur les pourtours du lac de Gabraoun, un ensemble oasien parmi les plus pauvres et les plus enclavés du Fezzan. En 1989, les autorités publiques décident de déplacer les populations vivant encore dans l'erg vers un nouveau village, Gabraoun Jedid ou dans les autres agglomérations du Wadi afin de les faire bénéficier de la redistribution de la rente, qui butait sur la localisation marginale de Gabraoun... et peut-être aussi pour mieux les encadrer. Le «nouveau Gabraoun» ressemble à s'y méprendre aux autres villages, composé de maisons cubiques, ocres, flanquées de deux petites cours mais il s'en distingue par des aménagements absents dans les autres villages, tels que les rues goudronnées. En outre, les autorités publiques, en prise à la réticence des chefs de famille à quitter l'erg malgré des conditions de vie difficiles, décident d'attribuer à chaque famille non seulement une maison mais aussi des lots de terres de 10 hectares, mis en valeur à partir de nouveaux forages sur des terres incultes du Wadi.

Brahim s'occupe, avec son frère, de l'exploitation familiale. Il n'est d'ailleurs ici qu'à temps partiel puisqu'il suit une formation agronomique dans un Institut Technologique de Sebha. L'exploitation est divisée en plusieurs parcelles. On trouve des oignons, de la luzerne, une palmeraie, des arbres fruitiers et un modeste cheptel ovin... pour la consommation familiale. Les débouchés de la production sont locaux et régionaux. Le succès de l'exploitation incite ces agriculteurs à mettre en valeur de nouvelles parcelles avec le soutien actif des autorités locales du congrès de base.

Mohamed a une connaissance plus fine du marché. Natif de Fjej, diplômé de l'Université, il occupe un poste de responsable local d'un secteur rural du Wadi. Grâce à son réseau de relations, il a une connaissance plus aiguë de l'évolution de la demande extérieure. Il dispose, avec son frère, de 30 ha de terres à mettre en valeur. Des forages profonds ont permis d'installer un système d'arrosage performant et le matériel agricole est récent. Mohamed présente ses terres comme l'exemple de ce qu'est le Wadi aujourd'hui, un espace agricole productif et orienté vers la demande du marché. Il justifie la 
présence de travailleurs étrangers par le temps que sa charge de Secrétaire de circonscription lui prend. Ce chef d'entreprise présente son père comme un paysan, attaché à sa terre, qui se livre encore au jardinage. Pour lui, dit Mohamed, le principe «donne ton fils avant de donner ta terre » vaut toujours, une époque révolue. On retrouve les productions de l'exploitation de Gabraoun Jedid ; les vignes en composent une part importante car le raisin est mûr en mai contre septembre sur la côte. Les profits tirés de cette agriculture de contre-saison sont importants.

Ces deux exploitants ont en commun le souci de la productivité et de la demande du marché. Un marché étendu non seulement au Wadi qui se transforme en une zone maraîchère mais aussi à l'ensemble de la Libye et même des marchés occidentaux. En effet, la faiblesse du dinar libyen et les difficultés liées à l'embargo ont incité des Tunisiens à jouer le rôle d'intermédiaires pour écouler une partie de cette production hors du territoire libyen. Ces entreprises agricoles, fondées à partir des lots distribués par l'Etat, constituent aujourd'hui le pivot du dynamisme agraire du Fezzan selon des principes propres à la Libye, à savoir un propriétaire libyen, des travailleurs étrangers, le choix de productions rentables. Mais l'égalitarisme, qui constituait initialement la base de la redistribution des terres sous forme de lots, est mis à mal par ces nouveaux exploitants car les bénéfices qu'ils retirent de leurs ventes et les incitations des autorités locales à mettre en valeur les terres poussent les principaux entrepreneurs à étendre leurs propriétés par la mise en culture de nouvelles parcelles. Par ailleurs, l'intervention étatique a facilité la mise en place de véritables stratégies économiques dans un cadre familial, qui sont d'autant plus actives que ses membres sont bien insérés dans les rouages locaux de l'appareil d'Etat. La famille de Brahim est ainsi passée en une génération du statut de paysans pauvres soumis aux aléas de l'agriculture de subsistance à celui de classe moyenne à revenus d'origine agricole et étatique (pensions et subventions). En revanche, celle de Mohamed dispose des moyens financiers et relationnels suffisants pour construire une forme d'entreprenariat privé au sein duquel s'observent les caractères d'une accumulation de capital suffisamment conséquente pour permettre des investissements fonciers et immobiliers.

La famille d'Ali vit dans un centre urbain du Fezzan. Son installation y est ancienne comme l'atteste le fait que l'un 
des ksour porte leur nom. Du temps de la royauté, son grand-père travaillait dans le commerce international de produits alimentaires, une activité reprise par son fils. Elle permet à la famille de construire une vaste résidence. L'extension de l'habitat individuel public autour de cette maison facilite l'installation des différents enfants à proximité de la demeure du père.

A la fin des années 1970, la suppression du commerce privé fragilise leur situation. Mais l'oncle d'Ali et le beau-fils de ce dernier exercent des responsabilités administratives dans la baladiya et, pour sa part, Ali détient un poste de décision dans un Ministère. Cette famille de notables de «l'ancien régime » a donc opéré un glissement du commerce privé vers l'administration publique, choix nécessaire afin de conserver un important réseau de relations. Initiateurs précoces du mouvement de résidentialisation, ils possèdent désormais tous de vastes demeures et louent les domiciles qui leur avait été attribué par l'Etat à des immigrés travaillant à $\mathrm{Al}$ Gharayfa. Ils participent aussi $\mathrm{du}$ mouvement d'extension des terres agricoles. Sentimentalement, chacun reste attaché aux 2 hectares de terre familiale à proximité du ksar, dans la vieille palmeraie mais ce qui focalise désormais l'attention des pères de famille, c'est le domaine mis en valeur depuis peu à Ad Disa, dans le cadre d'un périmètre alloti. Les 35 hectares de terres y sont consacrés aux fourrages et à l'élevage d'ovins, vendus par la suite à Sebha.

Cet exemple illustre l'étroite association entre les mutations urbaine et rurale, gérées dans le cadre familial par des notables vigilants aux adaptations indispensables au maintien de leur statut malgré les soubresauts brutaux du système politique libyen.

Chez Azzedine, l'idée de stratégie familiale prend tout son sens mais elle vise ici au maintien d'un niveau de vie minimal ; toute aussi ancienne que celle d'Ali, sa famille vit dans un village. De modestes moyens financiers initiaux n'ont pas permis à ses membres de s'installer dans le lotissement d'Etat. Ils vivent donc dans la partie du village construite durant les années 1950, aujourd'hui 
très dégradée, comme quelques familles libyennes. La poursuite des cours à l'Université est considérée par Azzedine et son cousin comme une opportunité réelle de promotion sociale ultérieure. Ils doivent toutefois travailler pour assurer un apport aux revenus familiaux. Chacun d'eux tient donc une boutique, épicerie pour l'un, tabac pour l'autre. Leurs frères, plus âgés, sont fonctionnaires. Ils profitent de leur temps libre pour cultiver la vieille propriété familiale. Malgré l'aide financière de l'Etat, ils ne peuvent envisager de financer le forage indispensable à la création d'une parcelle sur les terres vierges.

La mise en relation de ces deux itinéraires familiaux est presque caricaturale tant tout semble les opposer. Elle permet de voir que l'égalitarisme de façade, promu à travers l'habitat et les exploitations agricoles publiques, n'a pas atténué le rôle déterminant des modes de relations sociales inscrits dans la durée. La place de chaque famille dans les hiérarchies sociales conditionne des trajectoires différentes dans le tissu urbain des agglomérations et une inégale propension à participer au mouvement d'extension des exploitations agricoles privées.

Le cadre familial paraît toujours constituer une structure incontournable même s'il est parfois considéré comme désuet et conservateur, souvent synonyme de statisme et d'enracinement local. Ce n'est qu'en se référant aux modalités selon lesquelles il fonctionne et ses membres l'organisent que l'on prend la mesure des remarquables adaptations dont il fait preuve, à la fois comme élément de protection de chaque membre mais aussi comme support indispensable aux stratégies des individus. La place prise par le cadre étatique varie considérablement selon les individus et les familles. Dans les cas de Brahim et Mohamed, malgré de fortes divergences, on constate que l'Etat initie et impulse une dynamique que saisissent ou dont bénéficient les individus comme élément de leur promotion sociale. En revanche, les exemples d'Ali et d'Azzedin permettent de pointer deux stratégies à l'œuvre, l'une d'insertion, difficile, à un espace où l'urbain se diffuse, l'autre contribuant à cette diffusion.

\section{GHADAMÉS, ENTRE LOCAL ET GLOBAL}

Nous mesurons quelques champs de l'action au niveau local. Il est plus délicat de les appréhender dans le cadre de relations avec d'autres niveaux. 
Les enjeux patrimoniaux autour de la médina de Ghadamès illustrent une autre facette de ces relations.

Ghadamès est l'archétype de la ville saharienne. Le patrimoine y est enjeu mais aussi instrument d'une politique dont les acteurs se situent à divers échelons, du local au mondial. Ghadamès est, en ce sens, un exemple marginal mais dont la portée permet de nuancer le rôle de l'un ou de l'autre des acteurs au profit des multiples relations qu'ils établissent entre eux. L'identité de Ghadamès se fonde sur la référence à un patrimoine qui distingue la ville de l'ensemble des agglomérations libyennes et même sahariennes, selon l'argumentaire de l'UNESCO. Cet élément est prégnant dans les discours et les actes de tous les acteurs impliqués.

L'UNESCO tend à valoriser certains sites et développe ainsi une logique patrimoniale à l'échelle mondiale. Ghadamès est, dans cette perspective, un « exemple éminent d'un habitat traditionnel représentatif d'une culture et qui est vulnérable sous l'effet de mutations irréversibles » (Icomos $\left.{ }^{18}, 1986\right)$. Le classement au patrimoine mondial contribue à donner un «label de qualité », à valeur universelle. Celui-ci se fonde sur le principe de sauvegarde de la tradition et du risque de sa disparition.

Le rôle de l'Etat semble limité, du moins si l'on se fie à ce qu'en disent les décideurs locaux. De ses services émanent la production des images de la ville, par l'intermédiaire de l'organisme étatique, le bureau général du tourisme. Ainsi, dans tout lieu susceptible d'être pratiqué par les touristes, restaurants, hôtels, agences de voyage, à Tripoli ou dans le Fezzan une ou plusieurs photographies de la vieille ville sont affichées. Celles-ci représentent des vues de la médina, qualifiée de «Perle du Désert » ou de « Trésor caché ». Un fascicule en français et en anglais, faisant la part belle à Ghadamès a même été édité récemment ${ }^{19}$ et rares sont les articles de presse consacrés à la Libye, de la revue Géo au quotidien Le Monde, qui n'évoquent pas le lieu. Cette promotion par l'image est complétée par l'installation d'un bureau chargé de la promotion de Ghadamès, ouvert depuis peu, et la tenue d'un festival annuel.

$\mathrm{Au}$ niveau local, une véritable organisation fonctionne. Elle est le fait de Ghadamsis travaillant pour l'organisme officiel ou dans les agences de voyages privées qui fleurissent dans le nouveau centre. Ils sont soucieux de maintenir en bon état une ville qu'ils pratiquent encore, quotidiennement

\footnotetext{
${ }^{18}$ Icomos, Argumentaire pour la nomination du site archéologique de Ghadamès à la liste du patrimoine Mondial de l'Humanité, ronéotypé, 1986, 3 p.

${ }^{19}$ Secrétariat au tourisme, Les cités du Sahara, Dar al anies, Misrata.
} 
pour le jardinage et l'élevage du petit bétail ou en été, comme le vantent les guides de voyage, estivale lorsque la chaleur diurne rend les logements de la nouvelle ville insupportables. Les rues couvertes de la médina deviennent alors un lieu de rencontre, de discussion, de sieste.

La dynamique de singularisation et de construction de l'identité constitue-t-elle une passerelle entre ces différents acteurs ? Le classement au patrimoine mondial de l'Humanité, proposé par la Jamahiriya Arabe Libyenne date de 1986. Il s'assortissait de recommandations, présentées dans un plan de gestion en trois parties (Icomos, 1986) : « la délimitation d'une zone de protection autour de la vieille ville, bien distincte de l'agglomération moderne dont la croissance devait être contrôlée; la sauvegarde des techniques et savoir-faire traditionnels pour assurer la conservation d'un bâti fragile dans le respect des formes et des matériaux ; le contrôle de l'oasis dont la survie est nécessaire à la compréhension historique et à l'équilibre écologique de la ville. Les systèmes traditionnels d'irrigation de la palmeraie devraient être l'objet de soins particulièrement attentifs ». La prise en charge pratique du projet devait relever de la Direction des Antiquités du Ministère Libyen de l'Education.

Il faut replacer ces décisions dans leur contexte, celui de la profonde mutation urbaine du territoire libyen durant les années 1970-1980. La croissance démographique du pays s'accompagnait d'une multiplication ou de l'extension des agglomérations. Dans le cas de Ghadamès, l'espace bâti passait de 32 à 167 ha entre 1966 et 1980, soit un quintuplement en 16 ans en moyenne alors qu'un doublement était prévu d'ici l'an 2000 (S. Qezeiri, 1989). On comprend, à l'appui de ces chiffres, que l'objectif principal était celui de la préservation du site d'une urbanisation rapide qui n'aurait pas manqué de précipiter sa détérioration.

Mais durant les années 1990, le ralentissement de la croissance démographique, la moindre intervention étatique dans la production urbaine et la dégradation de la géopolitique internationale et régionale ont relégué Ghadamès dans une situation périphérique : périphérie frontalière compte tenu des faible flux de circulation avec l'Algérie, composés d'immigrés originaires d'Afrique subsaharienne et d'Algérie, périphérie nationale avec la suppression des liaisons aériennes vers Tripoli. La ville, avec 7500 habitants, est moins peuplée qu'au siècle dernier, autant que dans les années 1940, si l'on exclut de ce décompte les Touaregs Ifoghas, fixés durant ces dernières décennies. Toutes les conditions étaient donc réunies pour que la ville entre à nouveau en léthargie. Pourtant, le nombre de visiteurs d'une année sur l'autre augmente rapidement : au début des années 1980, Ghadamès est une excursion que l'on peut effectuer sur 
autorisation spéciale à partir d'un voyage en Algérie ; durant la saison 1998, 4000 touristes y ont séjourné, et probablement plus de 10000 en 1999.

La croissance rapide du nombre de touristes est de toute évidence le déclencheur des actions en cours à Ghadamès. Le classement du site n'ayant jamais abouti à des décisions concrètes, le comité populaire local demande, en session du congrès général populaire, l'autorisation de prendre en charge l'organisation du tourisme à Ghadamès. Or, le ministre du tourisme est lui-même originaire de la ville. Les responsables concernés au sein du Congrès général contactent alors l'UNESCO qui conseille de fonder une association. Celle-ci fonctionne depuis trois ans sous la dénomination de «gestion et organisation de la vieille ville». La coordination en est assurée par un Ghadamsi, qui dirige aussi une agence de voyage à Tripoli.

Les membres de l'association s'en tiennent pour le moment à deux objectifs, rénover la vieille ville et la viabiliser. En effet, ils considèrent que la priorité est de permettre aux citadins et aux touristes de pouvoir vivre ou séjourner à l'intérieur du site dans des conditions conformes aux normes de confort des agglomérations libyennes actuelles. A plus long terme, les projets portent sur la création d'un pôle touristique international. Le point d'orgue de l'année touristique est indéniablement le festival de Ghadamès qui attire chaque année des touristes libyens mais aussi étrangers, dont quelques personnalités, afin d'accroître la médiatisation du site.

Cette valorisation du patrimoine bénéficie à l'ensemble de l'agglomération qui jusque là avait eu la trajectoire banale de beaucoup d'autres villes sahariennes. Au début du siècle, Ghadamès présente une organisation oasienne «classique ». Le village et les palmeraies forment un ensemble ramassé, enserré dans des remparts, afin de se protéger des attaques extérieures. La présence coloniale se matérialise par la construction d'un fort et de quelques services administratifs à proximité. Ce site périphérique s'étend avec la fixation des nomades Touaregs Ifoghas et aboutit à une dualité urbaine entre le ksar et le noyau récent colonial et nomade.

Cette dualité s'accentue lorsqu'une ville nouvelle est créée à partir du noyau colonial dans les années 1970. Le ksar se vide rapidement de ses habitants, le clivage ancien entre les deux tribus rivales de Ghadamès, Walid et Wazit, s'atténue et l'installation des Ifoghas s'accélère. La physionomie de Ghadamès devient alors en tout point identique à celle que l'on peut trouver dans les autres agglomérations du Sahara libyen. En 
somme, le processus d'urbanisation impulsé par l'intervention étatique produit une uniformisation spatiale et l'atténuation de la différenciation tribale et ethnique caractéristique des vieilles villes oasiennes.

Or, la croissance du nombre de touristes réactive la dualité morphologique. En effet, d'un côté, un site saharien est valorisé par son intégration dans des réseaux mondiaux de circulation mais aussi de catégorisation fondée sur l'universalité «labellisée » du patrimoine par l'UNESCO. Un petit pôle touristique se conforte le long des murailles de la médina, autour du centre d'information où se multiplient les agences de voyage, les boutiques d'artisanat et les restaurants. Alors que de l'autre côté, la ville nouvelle reste très dépendante de sa situation sur le territoire libyen qui détermine un type d'urbanisme étatique uniforme et se caractérise par un périphérisme lié à l'isolement géographique de Ghadamès, à 650 kilomètres de Tripoli et 300 de Nalout, la ville la plus proche. Le clivage spatial qui se dessine au plan local est donc l'émanation d'une double insertion à bien des égards paradoxale, dans un réseau international et dans une continuité territoriale.

Les élites locales ont pris conscience de ce décalage croissant et elles tentent d'y remédier en développant deux types d'actions, à partir de la logique patrimoniale, l'une envers la vieille ville, l'autre envers la nouvelle. Dans le premier cas, la volonté est d'habiter le patrimoine afin qu'il ne soit pas figé et ne se détériore pas. Si la pratique estivale de la vieille ville par les Ghadamsis est très répandue, il s'agit maintenant d'intégrer les deux parties en laissant la possibilité à ceux qui le désirent d'aller s'installer dans leur ancienne demeure ou de la transformer en pension touristique. La viabilisation consisterait alors en la mise en place d'une adduction d'eau et à l'électrification. La ville nouvelle se transforme aussi, notamment sous l'effet du regard extérieur sur la ville qui a conduit à la collaboration entre les différents acteurs. En effet, les Libyens en contact avec les touristes constatent que ceux-ci soulignent fréquemment que les palmeraies et les rues sont jonchées de déchets sans que les habitants semblent s'en soucier alors que cela nuit à la qualité du site. Il a été en conséquence décidé à Ghadamès de procéder à un entretien régulier des espaces publics et à la construction des habitations selon les normes esthétiques de la vieille ville.

A Ghadamès, les acteurs locaux sont très empreints d'une certaine idée de la citadinité, entendue ici comme élément de singularité, en opposition à l'urbanisation uniformisante du Sahara libyen. Ils ont pu, en s'appuyant sur leur patrimoine et un réseau de relations particulièrement efficace, agir à différents niveaux. L'exemple de Ghadamès est certainement marginal en 
Libye où la plupart du temps les mutations du tissu urbain se sont effectuées au détriment des centres historiques. En revanche, il renvoie à une situation commune à l'ensemble du Sahara, celle où les touristes s'attendent à voir tous les éléments constitutifs de l'image qu'ils se font du désert durant de brefs voyages. Il impulse en conséquence une demande en paysages touristiques à laquelle se prêtent les acteurs locaux, d'autant plus aisément qu'elle leur permet de faire valoir le rayonnement dont ils bénéficient au plan national

Malgré sa marginalité, l'exemple de Ghadamès montre à quel point le jeu des acteurs est déterminé par le contexte précis il se déploie. Ici, le poids d'une tradition d'ouverture vers l'extérieur, qui se traduit aujourd'hui par une présence notable des acteurs de la ville, et de l'image qu'ils en diffusent, dans les rouages de l'activité touristique en Libye, reste déterminant. 


\section{CHAPITRE 5 - LA FIN DU MODÈLE URBAIN ÉTATIQUE}

Le processus d'urbanisation déclenche évidemment une extension importante des tissus urbains dans les villages et les villes du Fezzan. A peu près partout, cette extension s'effectue en trois étapes, des ksour, les villages sahariens traditionnels, aux formes étatiques nées durant les années 1970 jusqu'à la situation actuelle caractérisée par une diversité croissante des tissus urbains, au point que l'on puisse dégager un schéma commun d'évolution des formes, à la fois grille de lecture diachronique et synchronique. La rupture majeure dans cette évolution tourne autour du rôle de l'Etat, qui a créé les agglomérations actuelles puis s'est progressivement désengagé de ses fonctions de bâtisseur et de régulateur de leur croissance. Les mobilités et les hiérarchies sociales qui s'opèrent, rendues un temps peu visibles par la force du mouvement d'uniformisation urbaine, tendent désormais à marquer de plus en plus nettement les tissus urbains. Les agglomérations se fragmentent et deviennent ainsi dans leur morphologie, des observatoires du changement social et de dynamiques d'appropriation, individuelles et collectives, du bâti.

\section{LES ÉTAPES DE LA CROISSANCE}

En 1966, à l'exception de Sebha, le Fezzan ne compte que des villages, des hameaux et des centres ruraux de quelques centaines d'habitants. La faible diversité de l'habitat est donc marquée puisque dans la majorité des cas, les populations vivent encore dans les ksour ou bien dans les villages construits avec l'aide des autorités publiques durant les années 1950 et 1960. Ces villages sont peu diversifiés, mais aussi, peu étendus car le ksar se structure en un ensemble d'habitat individuel groupé. Cette organisation éclate sous l'effet de politiques d'aménagement volontaristes et les villages connaissent désormais des trajectoires variables selon la place qui leur est dévolue dans les hiérarchies urbaines et le dessin que les bureaux d'études chargés de leur conception en avaient faits. Les quatre agglomérations sur lesquelles s'appuient les analyses qui suivent reflètent la construction d'une trame urbaine hiérarchisée. A partir des années 1980, Sebha conforte sa place de centre régional du Fezzan, Oubari chapeaute l'ensemble du Wadi Al Haya, la principale vallée de la région, tandis qu'Al Gharayfa retrouve son rôle de bourg à l'échelle de la partie occidentale de cette vallée. Germa n'occupe pas de fonctions administratives notables mais l'importance du tourisme lui confère une place à part. 
En 1981 (tab.4), on prend la mesure de ce qu'est le modèle urbain étatique puisque de la moitié aux huit dixièmes de l'habitat est construit dans le cadre des plans d'aménagement. Au plan de l'extension spatiale, l'habitat public domine donc largement les formes d'initiative privée et à l'intérieur de cette première catégorie, les logements individuels l'emportent sur les collectifs ${ }^{20}$. Les autres formes d'habitat correspondent aux anciens villages, ksour ou noyaux construits durant les années 1950. Leur part dans le total est extrêmement variable mais toujours notable. Le résidentiel privé, très marginal, épars et relativement ancien, témoigne pour les cas relevés sur le terrain de la présence de familles de commerçants enrichis avant la nationalisation du commerce. La généralisation de l'urbanisation s'est donc accompagnée de l'extension du modèle étatique, contribuant à cette uniformisation si caractéristique des paysages urbains sahariens.

\section{Types d'habitats : les définitions et leurs limites}

Pour évoquer les différents quartiers des agglomérations, il convient d'utiliser des termes reconnus. Nous retenons la proposition de Pierre Signoles (1999) qui distingue des formes réglementaires, « où les règles d'urbanisme et les règles de construction sont peu ou prou respectées... » et des formes non réglementaires, qui «ne respectent pas les règles édictées par la législation et la réglementation en vigueur ».

Pour autant, l'opposition entre le réglementaire et le non-réglementaire n'a pas dans le Sud libyen de validité réelle car les autorités n'établissent pas de ligne juridique étanche entre ces deux formes. A Sebha, par exemple, l'installation de réfugiés tchadiens, avec l'aval du pouvoir central, nécessite la construction de centaines de logements mais la demande est telle que les autorités locales ne peuvent faire face. Un noyau que l'on pourrait qualifier, selon une formule barbare, de «légal, non-réglementaire et sous-intégré » se met en place : légal, on vient de voir pourquoi, non-réglementaire car construit selon des principes qui dérogent aux règles urbanistiques en vigueur, sous-intégré car ne bénéficiant

\footnotetext{
${ }^{20}$ Certes, le déséquilibre est accru par le mode de calcul puisqu'en mesurant les surfaces, on surestime forcément le premier au détriment du second. Toutefois, le choix d'un modèle est évident car, à l'exception de Sebha, le collectif coïncide généralement avec des familles élargies. Il s'agit donc aussi d'habitat individuel, adapté à la demande des populations.
} 
pas des mêmes équipements collectifs que le reste de l'agglomération. Ce contre-exemple montre les paradoxes de la catégorisation trop stricte. Des règles d'urbanisme sont édictées et globalement respectées par ceux qui désirent construire une résidence individuelle ; c'est peut-être plus l'écart à ces règles qui permet d'opérer une distinction.

Nous avons intégré sous la formule de non-réglementaire l'habitat insalubre car il ne compose qu'une infime partie des agglomérations. De même, l'extension du résidentiel et du collectif public a entraîné le déclassement des formes antérieures d'habitat dans la catégorie de non-réglementaire pour plusieurs raisons; d'une part parce que l'accès généralisé à de meilleures conditions de confort dans les nouvelles constructions a marginalisé les vieux villages ; d'autre part, pour les Libyens les plus modestes, les migrants et les nomades, ces noyaux d'habitat partiellement abandonnés ont souvent constitué la seule possibilité de logement. L'extension du non-réglementaire correspond donc à une insertion par défaut dans la ville : on s'installe ici car on ne dispose pas des moyens afin de s'installer ailleurs.

Les ambiguïtés que génère une division fondée sur les seuls critères juridiques sont nuancées ici par la distinction entre public et privé. C'est l'occasion de pointer pour chaque date les acteurs prédominants de la construction et d'introduire des nuances qui appellent à une lecture sociale des formes de l'habitat. Il nous a, par exemple, semblé nécessaire de distinguer sous le terme $\mathrm{d}^{\prime}$ « habitat mixte » les quartiers où résidentiel public et privé sont juxtaposés. Si du point de vue du clivage réglementaire/non-réglementaire, cette distinction n'a pas de sens, elle permet en revanche de mettre l'accent sur des indices du changement social (cf. ch.8). 
Tableau 4 : Les types d'habitats, essai de mesure (en pourcentage de la surface totale d'habitat) ${ }^{21}$

\begin{tabular}{|l|l|c|c|c|c|c|c|c|c|}
\hline & & \multicolumn{3}{|c|}{$\begin{array}{c}\text { Gl } \\
\text { Gharayfa }\end{array}$} & \multicolumn{2}{c|}{ Germa } & \multicolumn{2}{c|}{ Oubari } & \multicolumn{2}{c|}{ Sebha } \\
\hline $\begin{array}{l}\text { Habita } \\
\text { t }\end{array}$ & Types & $\begin{array}{c}198 \\
1\end{array}$ & $\begin{array}{c}200 \\
0\end{array}$ & $\begin{array}{c}198 \\
1\end{array}$ & $\begin{array}{c}200 \\
0\end{array}$ & $\begin{array}{c}198 \\
1\end{array}$ & $\begin{array}{c}200 \\
0\end{array}$ & $\begin{array}{c}198 \\
1\end{array}$ & $\begin{array}{c}200 \\
0\end{array}$ \\
\hline Public & Individuel & 55 & 46.6 & 48.7 & 26.2 & 45.6 & 21.6 & - & 31.2 \\
\hline & Collectif & 26.5 & 21.9 & 17.9 & 8.4 & 10.5 & 5.8 & - & 15 \\
\hline Privé & Résidentiel & 4.1 & 18.1 & - & 26 & 1.3 & 3.6 & - & 10 \\
\hline & $\begin{array}{l}\text { Non } \\
\text { réglementair } \\
\text { e }\end{array}$ & 14.4 & 13.4 & 33.4 & 39.4 & 42.6 & 48.6 & - & 24.3 \\
\hline Mixte & $\begin{array}{l}\text { (résidentiel + } \\
\text { public) }\end{array}$ & - & - & - & - & - & 20.4 & - & 19.5 \\
\hline Total & & 100 & 100 & 100 & 100 & 100 & 100 & - & 100 \\
\hline
\end{tabular}

Source : mesures sur cartes, planches d'atlas et relevés de terrain

Le rythme de l'extension spatiale ralentit entre 1981 et 2000 mais en même temps, il change dans ses modalités (tab.5). Plus que de mutation, il faudrait parler de nouvelle rupture dans les taux de croissance des types de bâti. L'ensemble du secteur public de la construction, qu'il s'agisse d'habitat et d'équipement, stagne alors qu'il contribuait quasiment en intégralité à la croissance entre 1966 et 1981. Le relais est désormais pris par le résidentiel privé, le non-réglementaire, le mixte en ce qui concerne l'habitat, le commerce et le tourisme pour le bâti fonctionnel. Initialement d'initiative publique, les agglomérations traduisent désormais l'emprise croissante d'acteurs privés, individuels ou collectifs, sur l'espace urbain. Ce basculement doit pourtant être observé au regard de la structure dominante. Le passage d'acteurs publics à des acteurs privés correspond à un rééquilibrage de l'action sur l'espace, sans doute aussi à un mouvement de réappropriation locale selon diverses modalités.

Tableau 5 : Taux de croissance du bâti entre 1981 et 2000 (en \% par an)

\footnotetext{
${ }^{21}$ Les mesures des différentes formes d'extensions urbaines ont été effectuées à partir d'éléments très disparates. Les cartes et les photographies aériennes les plus fiables datent de 1981 et sont issues d'un document sur l'aménagement du territoire de 1984. Le dernier ne concerne que le Wadi Gharbi, nous n'avons donc aucune information à propos de Sebha. Pour l'an 2000, nous avons procédé par relevés de terrain à partir des documents dont nous disposions, c'est-à-dire ceux de 1981, ainsi qu'un plan d'aménagement de Sebha. Les inévitables approximations qui en découlent nous ont conduit à privilégier les valeurs relatives qui donnent des ordres d'idée vraisemblables, aux superficies en hectares dont l'utilisation serait trop imprécise.
} 


\begin{tabular}{|l|l|c|c|c|}
\hline & & Oubari & Germa & Al Gharayfa \\
\hline Habitat public & Individuel & 0 & 0.7 & 0.3 \\
\hline & Collectif & 0.8 & 0 & 0.2 \\
\hline Habitat privé & Résidentiel & 9.9 & 100 & 9.4 \\
\hline & Non-réglementaire & 4.7 & 4.9 & 0.8 \\
\hline & Mixte & 100 & - & - \\
\hline Autre & Equipement & 0.2 & 0.9 & 0.5 \\
\hline & Commerce & 1.4 & 7.7 & 3.7 \\
\hline & Tourisme & 0 & 2.4 & - \\
\hline
\end{tabular}

Source : mesures sur cartes, planches d'atlas et relevés de terrain

La dynamique de résidentialisation est très marquée même si les mesures ne permettent pas réellement de cerner l'ampleur du mouvement. L'amélioration de l'habitat individuel public, qui relève de cette dynamique, ne peut-être que difficilement appréhendée par ce biais, contrairement aux deux autres modèles, mixte et résidentiel privé, directement mesurables sur les images. Ils contribuent à accentuer le clivage entre les petits centres et les plus importants, Oubari et Sebha. En effet, si partout les deux premiers modèles s'affirment, c'est de manière bien inégale. Par un paradoxe apparent, cette extension concerne essentiellement Al Gharayfa, Germa et Takarkiba. Dans les deux principales villes, le modèle mixte domine largement en terme de croissance et y correspond au cinquième de l'ensemble de l'habitat.

Partout, l'habitat non-réglementaire se développe rapidement, à l'exception d'Al Gharayfa puisqu'il correspond selon les lieux au moins au quart et jusqu'à la moitié de l'habitat. Peut-être faut-il revenir sur les évolutions différenciées du résidentiel et du non-réglementaire. Le premier est très récent, quasi-absent du tissu urbain en 1981, contrairement au second. Précisons que nous englobons les ksour dans cette dernière catégorie car l'extension de l'habitat public contribue à leur déqualification. En revanche, entre 1981 et 2000, l'évolution du non réglementaire correspond à l'autoconstruction, complétée par des formes d'habitat spontané à partir de ses noyaux ksouriens ou dans les interstices du tissu urbain récent.

Ces développements d'autres formes d'habitat témoignent de la limite du modèle uniforme comme à $\mathrm{Al} \mathrm{Gharayfa} \mathrm{où} \mathrm{la} \mathrm{croissance} \mathrm{est} \mathrm{due} \mathrm{pour} 60$ $\%$ au résidentiel privé et à Oubari où le non-réglementaire y contribue pour moitié. L'habitat non-réglementaire ne satisfait évidemment plus les catégories qui s'enrichissent mais sa part reste très insuffisante pour les couches les plus modestes et les migrants qui se voient en conséquence 
contraints de se loger par d'autres biais, notamment en recourrant massivement à l'autoconstruction.

Les équipements ont pour l'essentiel été construits en même temps que les nouveaux villages comme le montre la situation en 1981, comme l'atteste une extension presque nulle depuis. Mais l'espace affecté aux différentes activités connaît parfois une dynamique plus marquée, tels le tourisme à Germa (14\%) et le commerce à Al Gharayfa (13\%). L'effet de rééquilibrage consécutif à la prépondérance de l'action publique joue de toute évidence à plein, mais de manière plus différenciée que dans l'habitat.

\section{UN MODÈLE D'ÉVOLUTION COMMUN ?}

Takarkiba, Al Gharayfa, Oubari et Sebha diffèrent par leur taille, leurs fonctions mais présentent un modèle d'évolution en partie commun au plan morphologique, comme l'atteste le décryptage des formes. En revanche, leur trajectoire diffère totalement, permettant ainsi de comprendre comment, en quelques décennies, des villages sans fonctions historiques notables jusqu'au $\mathrm{XX}^{\mathrm{ème}}$ siècle ont pu si diversement se transformer selon un schéma que l'on peut diviser en quatre étapes chronologiques (tab.6).

Tableau 6 : Trajectoires urbaines

\begin{tabular}{|l|l|l|l|l|}
\hline Etapes & Takarkiba & Al Gharayfa & \multicolumn{1}{|c|}{ Oubari } & \multicolumn{1}{|c|}{ Sebha } \\
\hline Précoloniale & $\begin{array}{l}\text { Ksar - } \\
\text { jardins }\end{array}$ & $\begin{array}{l}\text { Marché local } \\
\text { Ksour-jardins }\end{array}$ & $\begin{array}{l}\text { Lieu d'échange } \\
\text { nomades-agriculteurs }\end{array}$ & $\begin{array}{l}\text { Ksour - } \\
\text { jardins }\end{array}$ \\
\hline Coloniale & Néant & Néant & Poste militaire & $\begin{array}{l}\text { Principal } \\
\text { centre } \\
\text { militaire et } \\
\text { administratif }\end{array}$ \\
\hline $\begin{array}{l}\text { Rôle des } \\
\text { populations }\end{array}$ & $\begin{array}{l}\text { Emigration } \\
\text { et exode } \\
\text { rural }\end{array}$ & $\begin{array}{l}\text { Emigration et } \\
\text { exode rural }\end{array}$ & Fixation des nomades & $\begin{array}{l}\text { Réceptacle de } \\
\text { l'exode rural }\end{array}$ \\
\hline $\begin{array}{l}\text { Intégration } \\
\text { nationale }\end{array}$ & $\begin{array}{l}\text { Nouveau } \\
\text { village }\end{array}$ & $\begin{array}{l}\text { Nouveau } \\
\text { village } \\
\text { Promotion } \\
\text { administrative }\end{array}$ & $\begin{array}{l}\text { Ville construite } \\
\text { ex-nihilo }\end{array}$ & $\begin{array}{l}\text { Centre } \\
\text { administratif } \\
\text { régional }\end{array}$ \\
\hline $\begin{array}{l}\text { Situation } \\
\text { actuelle }\end{array}$ & $\begin{array}{l}\text { Village, } \\
\text { tourisme }\end{array}$ & Centre rural & Centre micro-régional & $\begin{array}{l}\text { Centre } \\
\text { régional et } \\
\text { saharien }\end{array}$ \\
\hline
\end{tabular}

Durant la situation précoloniale, le centre le plus important était $\mathrm{Al}$ Gharayfa, tant en nombre d'habitants que par ses fonctions d'échange. 
Germa et Sebha présentaient des caractères communs, ceux de villages associés à une palmeraie et Oubari n'était qu'un puits, parfois lieu de négoce entre les Touaregs et les oasiens du Wadi Al Haya, un point nodal, très faiblement peuplé à la croisée de pistes importantes. La décadence de la première moitié du $\mathrm{XX}^{\text {ème }}$ siècle est une période de rupture durant laquelle Takarkiba et $\mathrm{Al}$ Gharayfa connaissent un exode rural marqué alors qu'Oubari et Sebha, après leur promotion administrative, voient leurs effectifs de population croître rapidement. A Sebha, chacune des quatre étapes ajoute à un processus cumulatif d'attractivité des populations et de renforcement du rayonnement qui se dessine durant le $\mathrm{XX}^{\text {ème }}$ siècle, faisant d'un village parmi d'autres une agglomération qui rayonne aujourd'hui sur tout le Sahara central. L'ensemble de villages oasiens du Fezzan laisse donc progressivement place à une trame de peuplement hiérarchisée où la répartition des fonctions, touristiques, commerciales, administratives différencient de plus en plus nettement les agglomérations.

Takarkiba résume les principales transformations de l'habitat dans le Sud libyen. Ce village du Wadi Al Haya, en bord de route, compte environ 700 habitants. Ici, la couleur dominante est l'ocre car elle revêt les bâtiments construits durant les années 1980. On mesure ainsi la prépondérance du poids de l'Etat puisque la majorité des équipements et des logements individuels en relèvent. Ce «cas d'école» nous permettra de mieux comprendre les évolutions des autres agglomérations.

Le ksar, village saharien traditionnel, correspond au noyau initial. L'habitat y est groupé et les maisons qui donnent sur l'extérieur en constituent la barrière défensive. Le matériau utilisé est local, terre crue et troncs de palmiers. Durant les années 1950, un second village est construit dans la palmeraie à l'initiative des autorités publiques. Sa structure est identique à celle du ksar : la mosquée est au centre et les maisons autour, construites dans des matériaux identiques. En revanche, une velléité de planification se traduit par un quadrillage des rues qui ne sont plus « tortueuses » comme dans le ksar mais droites.

La mise en œuvre des plans d'aménagement et de rénovation rurale après l'arrivée au pouvoir de Mouammar Kadhafi entraîne une rupture. Partout des villages sont construits par des entreprises étrangères, selon des normes identiques, le quadrillage des rues est de rigueur et les maisons standard. Mais à la différence du déplacement du ksar vers le premier village planifié, cette fois, seule une partie des villageois en bénéficie car les familles déménagent au fur et à mesure de la construction des maisons, processus qui n'est d'ailleurs toujours pas achevé. La succession 
des sites entraîne donc la juxtaposition de trois noyaux : le ksar est vide, le village de l'après-guerre en passe de l'être et le troisième est le véritable village actuel.

Le village gouvernemental (photo. 2), au plan géométrique, aux maisons cubiques et aux rues rectilignes est donc le centre actuel de Takarkiba. En parcourant ses rues, on constate que la couleur ocre y est concurrencée par le ciment ou des couleurs plus vives, les maisons centrales ont été surélevées par leurs habitants et quelques-unes transformées en résidences vastes et ostentatoires. Ces dernières se multiplient même si la taille du village exclut la présence d'un véritable quartier résidentiel, malgré d'évidentes velléités.

Aujourd'hui, la dynamique de construction est palpable autour du noyau central. Au sud, les terrassements sont achevés, dans le prolongement géométrique du noyau. Un peu à l'écart, vers l'est, un lotissement de résidences étatiques est déjà habité et en voie de transformation. Projet postérieur au noyau villageois initial, celui-ci se compose de maisons de $250 \mathrm{~m}^{2}$, jardin compris, contre $500 \mathrm{~m}^{2}$ précédemment. En effet, les maisons du projet initial étaient vastes mais depuis, les subventions de l'Etat ont réduit et ceux qui veulent construire sur plus de $250 \mathrm{~m}^{2}$ le font à leur frais. On dispose donc d'un bon indicateur de la hiérarchisation sociale en cours et de ses effets spatiaux.

\section{Photographie 2 : Takarkiba, le modèle urbain étatique}

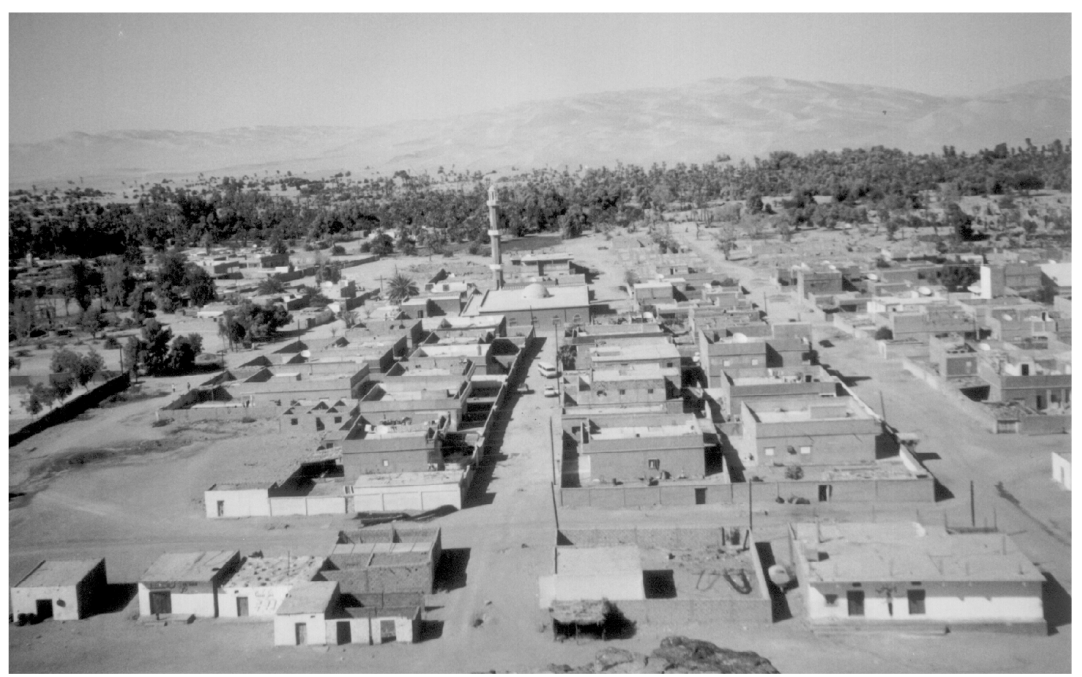


Pourtant, les indicateurs les plus utiles ne se perçoivent pas directement dans le paysage. Le plus flagrant est celui de la présence de Libyens dans le vieux village construit à la fin des années 1950. Ce dernier est aujourd'hui en grande partie vidé mais des familles s'y maintiennent, des étrangers s'y installent, essentiellement Nigériens, Maliens et Mauritaniens. Les maisons effondrées jouxtent celles qui sont maintenues, voire améliorées par leurs propriétaires. Certes, quelques habitants restent, par attachement à la demeure familiale, mais la plupart des autres vivent ici à défaut d'avoir pu acquérir une résidence gouvernementale.

C'est là une différence fondamentale entre la Libye et les pays voisins. Dans un ksar marocain, Mohamed Naciri (1986) décrit le déplacement du centre du pouvoir de l'intérieur du ksar vers l'extérieur, avec la création d'un centre administratif colonial, qui met à mal le système social. La contrainte communautaire s'atténue et les ksouriens s'installent dans des maisons individuelles. La communauté tente d'organiser ce déplacement afin qu'il concerne tous les villageois, que l'acte soit collectif et non individuel. Mais le plus souvent, ce sont les émigrés qui à leur retour prennent les premiers la décision de quitter le ksar, les autres habitants les suivent.

On peut donc lire la mobilité résidentielle comme une mutation sociale où les choix du groupe laissent place à ceux de l'individu. Cet élément ne suffit pourtant pas à expliquer tous les changements comme permet de l'étayer le cas de Takarkiba. Les deux premiers déplacements concernent l'ensemble des villageois, « la sortie du ksar » durant les années 1960, puis le déplacement vers le village gouvernemental durant les années 1970. Dans les deux cas, les autorités publiques ont encouragé ce mouvement en construisant une nouvelle structure, alors qu'à Tinjad, Mohamed Naciri souligne que le mouvement initial est le fait d'individus qui, après un temps passé hors du village en émigration, ont constitué un pécule afin de construire une résidence individuelle.

En Libye, l'Etat s'est substitué à ce mouvement individuel, donnant à la communauté les moyens d'assumer un déplacement collectif et donc de préserver la cohésion du groupe. Aujourd'hui, la situation diffère car l'étroite association entre la mobilité résidentielle et la fragmentation urbaine signifie aussi que le déplacement n'est plus tant communautaire qu'individuel. Le mouvement constaté au Maghreb a donc été différé. Ainsi, ceux qui n'ont pas pu bénéficier d'un logement individuel précédemment quittent le village construit durant les années 1950 pour aller s'installer dans le nouveau village au fur et à mesure de l'extension des lotissements publics. Par ailleurs, leurs logements vacants sont repris 
par la population immigrée qui vient s'installer dans le village. Enfin, l'amélioration des logements individuels des années 1970 et l'extension du résidentiel public constituent un dernier type de mobilité ou de dissociation de l'espace villageois uniforme.

Figure 9 : Oubari, la morphologie urbaine (1981-2000)



Oubari est l'exemple même de la « ville d'Etat », elle en illustre aussi la crise (fig.9). En 1944, le village compte moins de 700 habitants et malgré un doublement de la population en 20 ans, on peut considérer que la stagnation démographique perdure. Ce site de contrôle choisi par les autorités coloniales italiennes devient pôle régional dans le cadre des plans d'aménagement appliqués depuis l'Indépendance. Cette promotion administrative contribue à intensifier le rythme de la croissance urbaine, 2 500 habitants en 1973, 7200 en 1984 et 17000 en 1995, soit le taux de croissance intercensitaire le plus élevé du Sud sur l'ensemble de la période, nourri par la suite par l'installation de nouvelles populations sur un vaste 
périmètre agricole aux marges de la ville-centre puis la fixation de Touaregs sur leurs anciens itinéraires pastoraux et de Toubous venus après la guerre du Tchad.

Sur les trois sites villageois initiaux, deux seulement sont encore présents. Le troisième, au cœur de l'agglomération actuelle, a sans doute été détruit. La part du bâti d'initiative étatique est largement majoritaire, qu'il s'agisse de bâtiments administratifs ou de logements individuels ou collectifs. Ces trois formes se répartissent de façons plus ou moins concentriques, le long d'axes goudronnés. Au centre, l'habitat individuel domine dans un quartier homogène mais largement transformé par les habitants et autour, le collectif est plus important en superficie mais très dégradé. Curieusement, le résidentiel privé est proportionnellement peu présent par rapport à ce que l'on observe dans les autres agglomérations et ne forme pas de quartier homogène. On peut supposer que l'importance de l'intervention de l'Etat a contribué à la construction d'un nombre suffisant de logements mais aussi que la proximité du périmètre agricole et de nombreuses parcelles privées entrânent un mouvement de résidentialisation hors de la ville. L'ouest est dominé par une zone de bâtiments administratifs, hôpital, caserne, établissements scolaires et universitaires. Au final, cette répartition relativement rigide de l'habitat traduit la volonté de «zoning spatial » exprimée par les aménageurs à travers les plans urbains.

Le développement de l'habitat informel est plus significatif de l'état de crise de la ville. On compte au moins quatre sites : deux se greffent sur les villages initiaux, l'un à l'est, dans la palmeraie et le dernier au sud ainsi que de plus petits noyaux dans le tissu urbain central. La marque communautaire y est pourtant forte puisqu'une nationalité ou une aire régionale est majoritaire voire exclusive sur chaque site, Mauritaniens, Maghrébins, Toubous, Asiatiques (Pakistanais, Bangladeshi), Touaregs, Africains de l'ouest... et Libyens. La «communautarisation» des quartiers croît donc avec la diversité sociale et géographique des agglomérations.

La différence essentielle entre Takarkiba et Oubari, et plus généralement entre les villages et les principales villes du Fezzan réside dans l'apport permanent de populations extérieures à l'agglomération. L'émergence d'une individualisation des comportements, en opposition au groupe, ne s'y pose pas avec autant d'acuité puisque malgré la nette prédominance des familles touaregs en proportion de la population qui vient s'installer à Oubari, la préoccupation des autorités est plutôt de loger tous les arrivants. La fixation des nomades, par exemple, s'effectue autant 
en marge de l'agglomération que dans les logements publics qui la composent. A cette phase préliminaire de peuplement Touareg, se surimpose une seconde phase de migrants et de réfugiés. Les seconds prennent la place des premiers dans les noyaux villageois initiaux lorsque ces derniers accèdent à un logement d'Etat ou bien construisent leur propre demeure en se regroupant à nouveau par origine. Deux types de trajectoires animent donc le tissu urbain, celle des premiers arrivants, majoritairement libyens et celle des vagues de migrants plus récentes, généralement de migrants internationaux.

En définitive, l'urbanisation du haut vers le bas a pris dans le Fezzan tout son sens grâce aux moyens dont a disposé l'Etat. La diversification croissante du tissu urbain atteste de la fin du modèle urbain étatique, synonyme d'uniformité du bâti. Elle reflète aussi la complexité croissante des liens établis entre le Sud libyen, le reste du territoire national et les Etats voisins. Cette complexité se traduit par l'arrivée de populations de différentes origines géographiques, dont les stratégies résidentielles et l'insertion dans le tissu urbain varient fondamentalement. 


\section{CHAPITRE 6 - LA MUTATION RURALE SOUS L'INFLUENCE DE L'URBAIN}

Parmi les grands travaux initiés par l'Etat libyen durant les années 1970 et 1980, la rénovation agricole occupe une place de choix. Celle-ci visait à une moindre dépendance vis-à-vis du marché international et affichait en conséquence des objectifs de productivité mais surtout, elle participe d'un projet social et d'aménagement du territoire de grande ampleur. A l'échelle de la Libye, l'agriculture du Fezzan est évidemment négligeable avec à peine plus de $3 \%$ de la superficie agricole nationale et $12 \%$ des surfaces irriguées, mais au plan local cela se traduit dans les paysages par une continuité de l'activité agricole malgré de fortes disparités régionales. Il s'agissait d'abord de juguler les flux d'exode rural vers Sebha et le littoral en modernisant l'habitat et les pratiques agricoles et en diversifiant les autres activités économiques. Le marquage de l'espace fut une autre priorité qui s'est traduite par la volonté de maintenir des populations dans le Sahara, particulièrement dans les régions frontalières, en multipliant les périmètres agricoles. Quoique ancienne, l'activité agricole connaît donc depuis une vingtaine d'années des mutations importantes qui s'inscrivent dans un rapport de réciprocité avec le processus d'urbanisation, les deux s'effectuant conjointement. Pour cerner ce rapport, nous partirons d'une présentation des principales dynamiques rurales, puis des acteurs qui y participent avant d'en mesurer les conséquences spatiales dans le Fezzan.

\section{CRISE ET MUTATION D’UN ESPACE OASIEN}

La crise agricole est l'un des éléments les plus emblématiques de la « décadence » du Fezzan. Les pages de l'ouvrage de Jean Despois (1946) sont émaillées de descriptions sur le piteux état de l'agriculture et «l'aspect misérable » des exploitations bien que la mise en valeur des terres soit l'activité principale des Fezzanais. En revanche, les remarques de Marcel Mercier (1946) incitent à nuancer l'importance réelle de la crise, lorsqu'il précise que « l'impression d'abandon (des parcelles) tient moins à leur mise en valeur effective qu'à la méthode de jachère ». Certes, le Fezzan a connu une crise grave et durable mais elle n'explique pas à elle seule le faible dynamisme agricole régional.

Les superficies irriguées dans le Fezzan étaient inférieures à 1000 hectares en 1945 (J. Despois, 1946) ; elles sont d'environ 60000 hectares aujourd'hui. En 1962, la superficie totale cultivable de la région de Ghat 
est de 435 hectares (L. Eldblom, 1968), et de près de 4000 hectares en 1987. Ces chiffres conduisent à penser que l'extension des terres irriguées ne s'est pas effectuée durant les cinquante mais plutôt durant les trente dernières années.

Une approche par ensembles régionaux laisse apparaître une distribution relativement homogène des terres irriguées dont le cinquième se trouve sur chacun des deux axes du Wadi Ash Shati et de Mourzouq, un tiers dans le Wadi Al Haya, $15 \%$ dans la région de Sebha et $5 \%$ dans celle de Ghat. Les terres cultivables, c'est-à-dire celles dont la mise en valeur est possible, sont beaucoup plus inégalement réparties. Cette réserve foncière correspond à près de la moitié des terres de Sebha et du Shati, à un quart de celles de Mourzouq mais à seulement $10 \%$ des terres du Wadi Al Haya. On ne peut considérer ces chiffres comme totalement fiables mais en revanche, ils permettent de mesurer l'intensité de la mise en valeur à un moment donné.

A un niveau plus fin, on constate que la moitié des terres irriguées est concentrée dans les trois secteurs d'Al Gharayfa, Sebha et Brak. Le cas de Brak est un peu particulier puisque sur une aire réduite, 15 kilomètres de rayon, sont associés une agglomération moyenne, plusieurs villages et un périmètre agricole étatique. Néanmoins, la distinction des deux principales agglomérations n'est pas anodine puisque si les terres offrent un potentiel que l'on ne retrouve pas ailleurs, c'est aussi l'indice de l'intensité de la périurbanisation agricole. Il n'est pas non plus surprenant de noter la présence d'Al Gharayfa parmi les trois principales annexes. C'est même là une remarquable continuité historique puisque les terres les plus fertiles du Fezzan s'y trouvent, les plus convoitées, depuis toujours, par les propriétaires fonciers des trois vallées centrales. En définitive, le Wadi Al Haya et la région de Sebha constituent les zones agricoles pionnières.

Jean Lethielleux (1948) parle d'environ 2000 jardins pour l'ensemble du Fezzan dans les années 1940 et retient le chiffre de 2300 hectares, soit une superficie moyenne de 1,15 hectares. Aujourd'hui, la plupart des exploitations privées comptent de 5 à 40 hectares en irrigué. L'Etat a largement contribué à cette augmentation de la taille moyenne des exploitations par une série de plans d'aménagement, de la moitié des années 1960 à la moitié des années 1980. La réorganisation des terres dans le cadre des projets familiaux s'est accompagnée d'une multiplication des exploitations dont la superficie varie de 5 à 14 hectares, puisqu'elles composent en 1987 du cinquième au quatre-cinquièmes du nombre total des exploitations selon les lieux. De profondes disparités spatiales opposent les régions de Ghat et du Shati, peu touchées, à celles du Wadi Al 
Haya et de la dépression de Mourzouq, où l'action de l'Etat fut la plus importante. Ces disparités se retrouvent dans les vallées, notamment entre Oubari, où deux tiers des exploitations se situent dans cette fourchette et $\mathrm{Al}$ Gharayfa, nettement moins touchée. Or, Oubari est un noyau de peuplement récent alors qu'à $\mathrm{Al}$ Gharayfa, vieille région agricole, le morcellement et la petite taille des propriétés prédominaient avant l'intervention étatique. Ces exemples permettent de pointer les limites de l'action de l'Etat, très forte dans les espaces peu peuplés mais en butte à la gestion coutumière du foncier rural dans les vieux foyers de peuplement.

Culture autrefois prépondérante du Fezzan, le palmier dattier demeure aujourd'hui dans le Sahara libyen l'indice d'une mise en valeur traditionnelle des jardins. Malgré quelques projets de rénovation et d'introduction de nouveaux plants, les espèces les plus recherchées, telle la deglet nour, sont absentes. Notons que Marcel Mercier (1946) s'en étonnait déjà après la seconde guerre mondiale et sans doute aussi les autorités coloniales italiennes puisqu'elles tentèrent, sans résultat, d'en développer l'exploitation durant les années 1930. Les dattes servent aujourd'hui à la consommation locale et nationale, à la production d'une confiture et à la nourriture du bétail.

La répartition des palmiers dattiers révèle la forte corrélation entre l'urbain et la route (fig.10). Dans près de la moitié des annexes, le palmier dattier correspond à plus de $85 \%$ du total des arbres fruitiers. Or ces annexes sont en situation périphérique par rapport à l'axe routier principal. On a là une confirmation de l'étroite association entre les choix de production et les infrastructures, déjà constatée à propos du remembrement des parcelles.

L'importance du raisin est exactement inverse de celle du palmier dattier (fig.10). Elle est l'indice de l'émergence d'un marché urbain de consommation car le raisin de table fait partie des nouvelles cultures à forte valeur ajoutée. Sa répartition fait apparaître plus nettement encore que d'autres productions l'importance de l'axe central, de Sebha à Oubari, dans l'implantation des cultures périurbaines. La localisation de long de cet axe garantit un transport rapide des denrées fragiles vers les foyers de consommation proches comme Sebha, plus lointains comme la Tripolitaine.

Une dissociation assez nette entre deux types d'espaces ruraux se dessine donc : ceux qui correspondent aux principales villes où la périurbanisation transforme leurs périphéries et ceux qui furent remodelés dans le cadre de la rénovation rurale associant remembrement des exploitations, orientation commerciale des cultures et urbanisation intense. 
La qualité du réseau routier national, le très bas prix de l'essence, la grande mobilité des personnes et des produits et l'intense recomposition du rôle des acteurs agissent ici comme des facteurs déterminants du dynamisme de l'agriculture privée dans son fonctionnement actuel. On devine pourtant à quel point la situation est plus ambiguë qu'il n'y paraît au premier abord dans la mesure où les rouages économiques sont entre les mains d'acteurs privés. Mais leur marge de manœuvre est d'autant plus forte que l'Etat garantit des infrastructures et un soutien déterminant à leur initiative.

\section{LES ALÉAS DE L'AGRICULTURE BUREAUCRATIQUE}

La politique rurale de l'Etat libyen vis-à-vis du Sahara s'articule durant les années 1970 et 1980 autour de deux objectifs : restructurer et rénover les vieilles régions d'agriculture oasienne et mettre en place quelques vastes périmètres de production, médiatisés et à forte charge symbolique, celle d'un idéal d'autonomie nationale.

C'est à partir des villages existants que sont généralement conçus les projets de peuplement étatiques. Ils ont une facette agraire, fondée sur la redistribution des terres cultivables et l'attribution aux exploitants, théoriquement les plus modestes, d'un lot de 6 à 10 hectares à mettre en culture, d'une fermette, d'une bergerie, d'un verger, d'un tracteur, d'une somme d'argent et d'un prêt sur vingt ans visant à rembourser le tiers des équipements fournis (C. Souriau, 1984). Mais au-delà des dotations individuelles, se profile un projet plus ambitieux car avec 100 à 200 propriétés construites dans chaque périmètre, ce sont souvent plus de mille personnes qui sont concernées, notamment à Oubari, Oum al Aranib, Brak, Fjej et Tahala. D'autant plus que chaque périmètre est assorti de la création d'un centre de services et de soins, une école, une mosquée, une poste, un atelier de maintenance, une coopérative, un laboratoire d'analyse agricole et un marché (M. O. Attir, 1985). La politique de rénovation rurale aboutit donc à multiplier et à moderniser les noyaux de peuplement villageois et micro-urbains.

Eléphants blancs de l'agriculture saharienne ou projets de production nécessaires à l'indépendance nationale, les grands complexes étatiques focalisent souvent -à tort ?- l'attention par la démesure des investissements qui leur ont été consacrés.

Le site de Maknoussa se situe au sud du Wadi Al Haya. Il se présente sous la forme de deux séries parallèles de pivots, de part et d'autre de l'axe routier en direction de Mourzouq, sur un plateau. A chacun des 90 pivots correspond un forage et la superficie totale est de 3900 hectares, soit 45 
hectares par pivot. Deux récoltes par an sont assurées, une de céréales d'hiver (blé et orge) et une de céréales d'été (maïs). La production est écoulée localement ou sert de fourrage sur place car une station pilote d'élevage d'ovins et de bovins vient d'être installée sur le site.

Figure 10 : Les dynamiques de l'agriculture dans le Fezzan

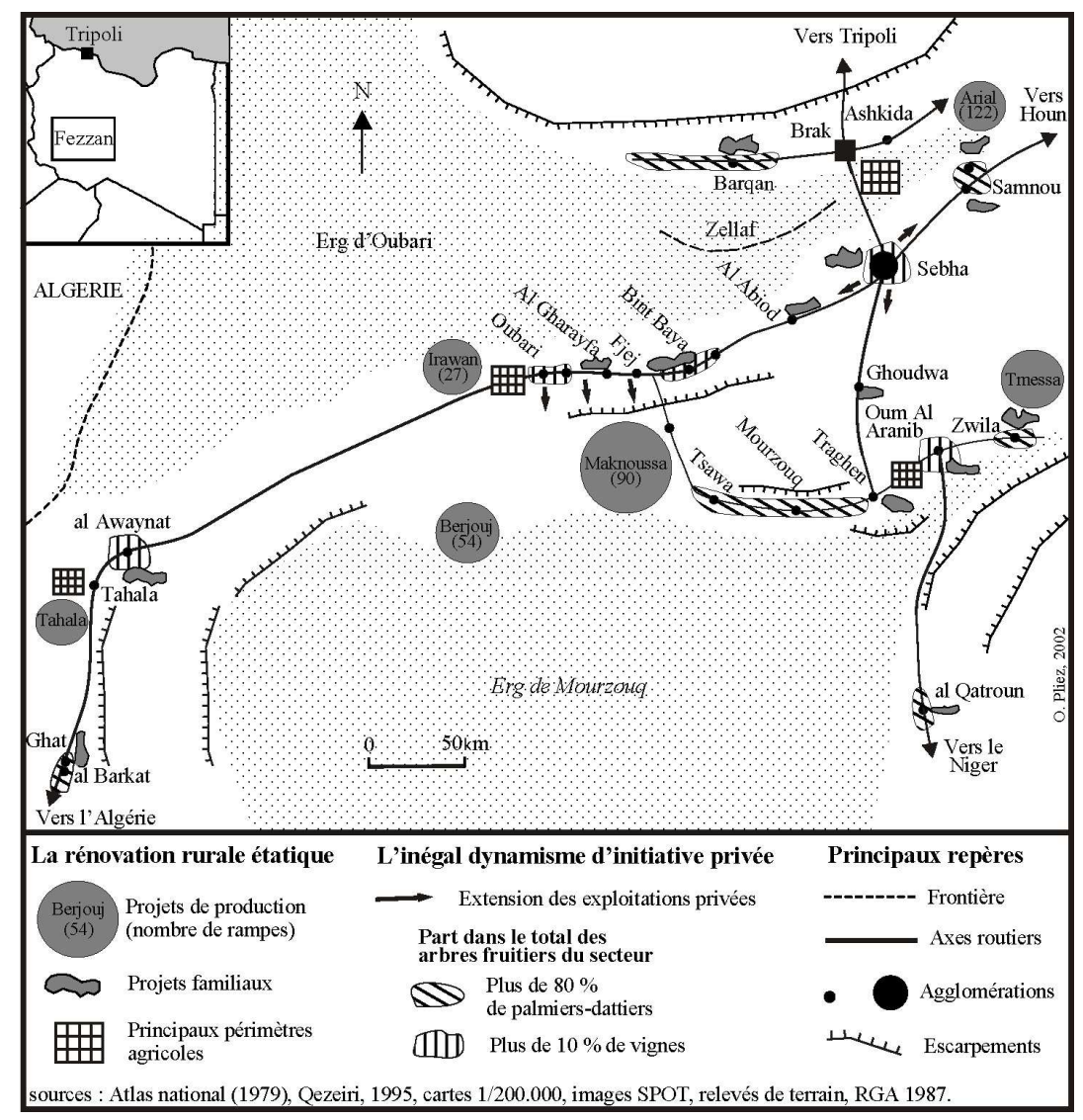

Malgré la proximité de deux vallées peuplées, le complexe de Maknoussa est marginal puisqu'il n'emploie que 200 salariés. De plus, les soucis de rentabilité ont conduit les responsables à réduire cet effectif dont la moitié vit dans les villages alentours et les autres résident sur place, en mobil-homes s'ils sont ouvriers ou techniciens ou bien dans un lotissement s'ils sont cadres. La rentabilité affichée du projet laisse sceptique car sans brise-vent, il est exposé aux vents de sable qui déposent des poussières sur 
les parcelles et sans engrais, ses rendements semblent nettement inférieurs à ceux des exploitations privées. Ce bilan mitigé contraste donc avec le dynamisme des parcelles de mise en valeur individuelle, très liée à la généralisation du processus d'urbanisation.

Dans le Fezzan, on recense six sites, Maknoussa (1978-1979, 90 rampes), Berjouj (1987-1989, 54 rampes), Irawan (1987, 27 rampes), Arial (1979-1980, 122 rampes), Tmessa et Tahala (fig.10). Chaque pivot a une superficie de 40 ha et l'on n'en dénombre au moins 300 pour cette seule région, soit plus que pour tout le Sahara algérien (Marc Cote, 1993). L'objectif est double, d'une part contribuer à l'autosuffisance alimentaire de la Libye dans le cadre plus idéologique des grands projets d'indépendance nationale, d'autre part contribuer au développement du Sahara libyen. La Fezzan Agriculture Authority (FAA) est créée avec pour objectif de gérer l'ensemble des sites régionaux. Une compagnie américaine fournit l'assistance et les conseils techniques, la majorité des employés viennent des villages alentours, les autres sont logés sur place dans des baraquements prévus à cet effet. Les productions diffèrent selon les saisons, blé en hiver, maïs en été mais aussi luzerne, ail.

Mais depuis le début des années 1990, le désengagement étatique touche les grands les grands périmètres construits durant les deux décennies précédentes. La privatisation des organismes chargés de la gestion des sites expose désormais la FAA à la double concurrence des produits importés et de la production agricole libyenne d'initiative privée. En outre, l'aide technique a pâti de la dégradation des rapports avec les Etats-Unis qui ont souvent fourni matériel et compétences. Dans ce contexte, la portée sociale et politique de l'agriculture bureaucratique s'est érodée, laissant place à des considérations plus pragmatiques, celles du marché. Or, sur les quatre sites gérés par la FAA, deux posent des problèmes de localisation, liés à l'âpreté des conditions naturelles, notamment, la salinité des sols et les vents qui assèchent les cultures et transportent la poussière de sable de l'erg. En outre, Irawan, à soixante kilomètres à l'ouest d'Oubari, pâtit de la découverte d'un champ d'exploitation pétrolier à 2,5 kilomètres vers lequel ont convergé la main-d'œuvre, les investissements et l'utilisation de l'eau des nappes fossiles. L'enclavement ajoute aux handicaps comme à Wadi Arial puisque le site décline à cause des difficultés liées à l'agriculture en zone sableuse et par suite des frais occasionnés par l'approvisionnement et la prise en charge des employés, toutes charges qui ne sont plus compatibles avec les objectifs de rentabilité. Au bilan, moins de la moitié des rampes d'aspersion fonctionnent. 
Les mauvais résultats financiers de Arial et Irawan sont difficilement compensés par ceux, positifs, de Berjouj et Maknoussa. Certes, des conseillers de pays proches de la Libye sont présents sur les sites, Egyptiens et Soudanais essentiellement mais les défaillances de la maintenance, la mauvaise qualité du matériel, rarement renouvelé depuis l'embargo, rendent la productivité aléatoire. Peu en prises avec les dynamiques qui traversent actuellement le Sahara libyen, ces projets risquent donc de décliner comme c'est déjà le cas pour celui d'Al Jawf dans le groupe d'oasis de Koufra, pourtant emblématique de cette agriculture bureaucratique.

\section{L'ESSOR DE L'AGRICULTURE PRIVÉE PÉRIURBAINE}

La combinaison des interventions publiques et de l'initiative privée caractérise désormais le rapport à l'espace rural dans l'ensemble du Fezzan. C'est là une rupture avec le principe de répartition des terres agricoles sous la forme de périmètres allotis assez similaire à celle constatée dans l'habitat, où l'arrêt de la construction d'individuel public a entraîné l'extension de nouveaux types d'habitat. En conséquence, l'extension des mises en valeur agricoles individuelles transforme les paysages du Fezzan, encore marqués par la trame déjà remodelée durant les années 1970 et 1980 sous impulsion étatique. Cette combinaison varie selon le degré d'intervention initial de l'Etat et le type d'urbanisation qui caractérisent le Sahara libyen: dans les vallées oasiennes, la micro-urbanisation linéaire le long de la route impulse un mouvement d'extension rapide de mise en valeur des terres vierges tandis qu'aux marges des villes d'Oubari et de Sebha se développe l'agriculture périurbaine.

Le dynamisme agricole et urbain du Wadi Al Haya (fig.10) se traduit par une mutation paysagère spectaculaire, dans une vallée où se concentre le tiers des terres du Fezzan sur une centaine de kilomètres. La route matérialise la limite entre deux formes de mises en valeur: les plus anciennes s'étalent en piémont de l'erg d'Oubari, là où la nappe phréatique se situait en moyenne à 3,6 m de profondeur (M. Mercier, 1946). Les formes plus récentes se situent de l'autre côté de la route et des villages, plus loin de l'Erg et des lieux de la vallée les plus densément peuplés et cultivés. On peut avancer au moins deux raisons pour expliquer ce mouvement. D'une part, contrairement à l'image encore répandue de la culture oasienne traditionnelle à trois étages -jardin, verger et palmeraie sur la même parcelle-, le Fezzan donne l'exemple de paysages agraires où la dissociation entre palmeraie et jardins est ancienne et où la multiplication 
de ces derniers conduit les propriétaires à chercher de nouvelles terres. D'autre part, l'acquisition de terres vierges ne pose pas de problèmes puisqu'elles deviennent automatiquement la propriété de celui qui les met en valeur. En revanche, l'importance de la mise en valeur est telle que les nappes superficielles de piémont d'erg s'assèchent; par conséquent le déplacement des cultures correspond aussi à une «quête » de l'eau là où les ponctions sont encore limitées.

Le Fezzan donne à voir une situation différente de celle qui prévaut en Tunisie, tant du point de vue des acteurs que des positions défendues par les autorités. La fixation des nomades constitue en effet dans le Sud tunisien, notamment dans le Nefzaoua, une impulsion essentielle dans l'extension des fronts pionniers agricoles sur les anciens itinéraires pastoraux; le modèle fixation/urbanisation/front pionnier domine. En Libye, au contraire, le front pionnier se situe à l'intérieur de la vallée, à l'initiative des villageois disposant des moyens financiers nécessaires et non des nomades ou semi-nomades installés récemment. Le témoignage d'un propriétaire d'Al Gharayfa illustre les modalités selon lesquelles ces exploitations sont créées :

Ce chef de famille possède 20 hectares. Il a d'abord délimité un périmètre sur les terres vierges à proximité du centre, a fait effectuer un forage à $55 \mathrm{~m}$ de profondeur puis a déclaré l'action aux autorités locales car si les terres vierges font partie des domaines de l'Etat, l'acte de mise en valeur entraîne leur privatisation au profit de celui qui l'effectue. Malgré l'insistance des responsables locaux, il n'a pas procédé à la rédaction d'un acte de propriété, préférant user ensuite du droit coutumier pour régler sa succession. En conséquence, il n'a bénéficié que d'une aide limitée puisque seule une partie des frais occasionnés par le forage lui a été remboursée. Le propriétaire participe occasionnellement à la mise en valeur de l'exploitation et partage la récolte pour moitié avec les deux Egyptiens qui y travaillent à temps complet. Comme dans la plupart des cas, ils ont privilégié la petite polyculture, céréales (blé et luzerne), une palmeraie, des arbres fruitiers (orangers, citronniers) et des légumes. Les fruits sont réservés par des grossistes de Sebha et les légumes sont vendus sur les marchés locaux. 
Le mouvement d'extension des parcelles privées s'inscrit donc très logiquement à la suite de celui impulsé durant les années 1970 et 1980 par les pouvoirs publics. Mais si le soutien financier est toujours conséquent ; en revanche, on passe d'un projet rural à vocation sociale à une incitation à vocation productiviste où les mécanismes fonciers sont gérés dans le cadre familial, en marge des règles que les autorités tentent d'instaurer.

Le périmètre d'Oubari est le type même des aménagements étatiques créés ex-nihilo, aujourd'hui en crise. Alors que les traces de foggaras indiquaient la présence d'une agriculture laissée à l'abandon depuis plusieurs décennies, la décision de promouvoir Oubari comme centre principal du Wadi Al Haya s'accompagne de la construction d'un des plus importants périmètres agricoles de peuplement du Sahara libyen à proximité de la ville.

Figure 11 : Oubari, un périmètre agricole étatique en crise



Ce périmètre (fig.11) s'étend sur 1800 hectares, découpé en 172 lots de 10 hectares chacun, et autant de fermettes, groupées par quatre. Des chemins rectilignes permettent de circuler entre les exploitations, séparées 
par des brise-vent de tamaris de $10 \mathrm{~m}$ de haut. Ce rationalisme contraste avec la faible intensivité des parcelles. Malgré la présence d'agriculteurs égyptiens et de manœuvres subsahariens, le projet agricole, construit « clés en main » pâtit d'un évident manque de maintenance. A cela plusieurs raisons, l'absentéisme des propriétaires, le sous-effectif salarié (un employé égyptien par exploitation), la faible mécanisation. Au total, du tiers au quatre-cinquième de la superficie des parcelles est mis en valeur.

Figure 12 : L’agriculture périurbaine autour de Sebha

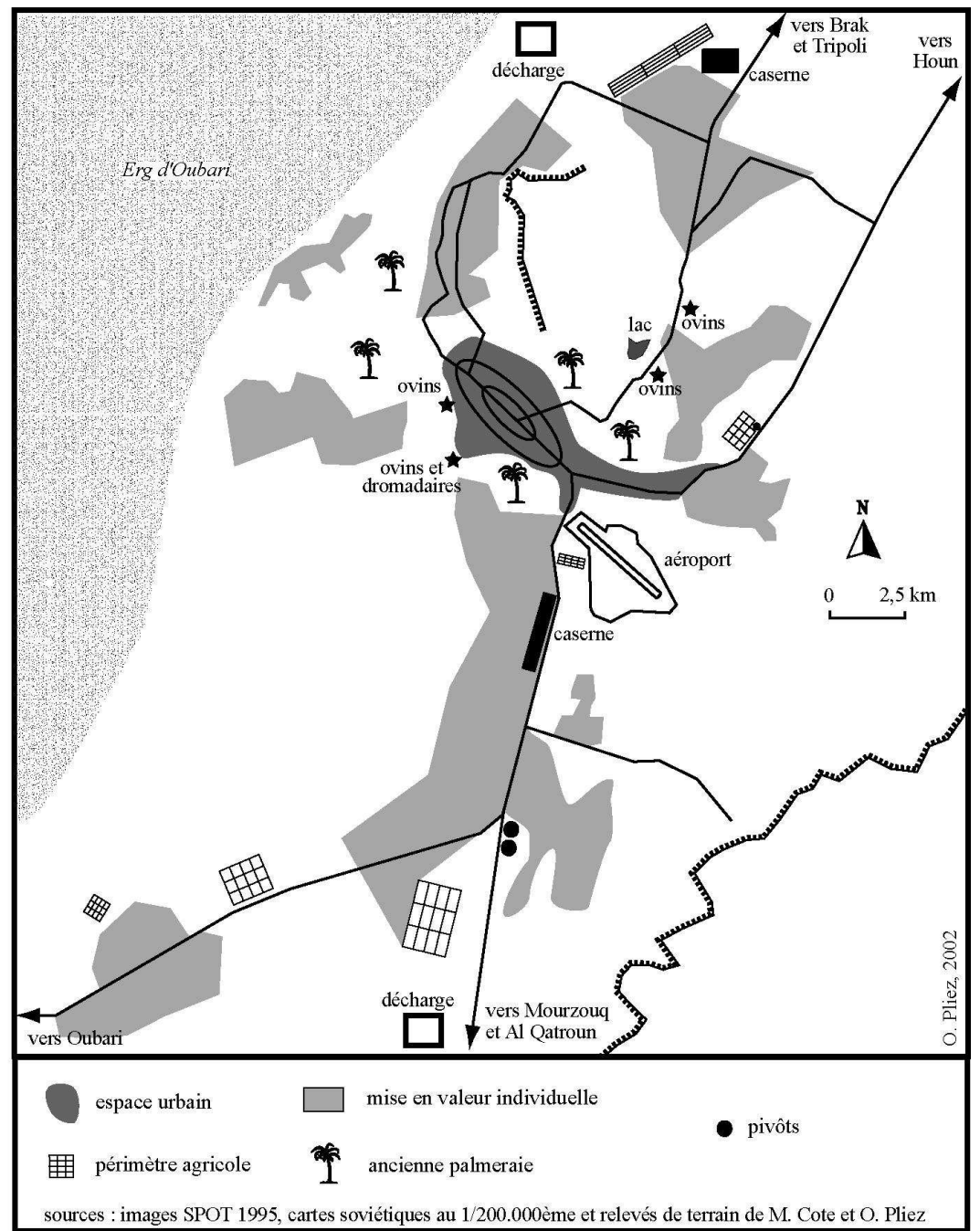


Les pressions extérieures sur ce périmètre sont fortes. Aux marges du projet, deux villages de fixation des nomades s'étendent, l'un de peuplement Toubou, l'autre plus important, de Touaregs. La proximité des brises vent qui délimitent le projet et séparent les exploitations, les équipements électriques et l'accès à l'eau sont une véritable aubaine pour ces pasteurs. Non seulement, ils utilisent le bois pour leurs besoins domestiques ou pour produire du charbon vendu dans les agglomérations mais de plus, ils profitent de la végétation pour les troupeaux qu'ils élèvent.

$\mathrm{Au}$ vu de l'exemple d'Oubari, le bilan des périmètres agricoles étatiques, tels Brak, Oum Al Aranib et Tahala, pour ne citer que ceux du Fezzan, est donc extrêmement mitigé et les empiétements révèlent, plus qu'ils ne suscitent, la crise. Ils devaient permettre aux familles d'agriculteurs ou de nomades qui se fixaient de bénéficier d'une complète autonomie tout en participant à la production agricole du Sahara. Malgré l'importance des moyens mis en œuvre, les problèmes sont multiples, techniques certes, mais aussi sociaux. Ce déclin contraste avec la vitalité des parcelles de mise en valeur privée jusqu'à la limite du projet et l'extension des villages de fixation des nomades, dont témoignent les multiples sites de fabrication de briques en terre crue. Ce n'est donc pas l'agriculture dans son ensemble qui est remise en cause mais certains choix de mise en valeur.

Le dynamisme agricole autour de Sebha (fig.12) s'apparente à celui du Wadi Al Haya. Là aussi, les vieilles palmeraies entourent la ville même si la pression urbaine s'exerce avec plus de force. Le plan d'aménagement à l'horizon 2000, établi au début des années 1980, prévoyait des extensions urbaines, qui dans les faits ont rapidement été suspendues car elles empiétaient trop sur les palmeraies. Les nouvelles exploitations se situent généralement le long des axes qui relient Sebha au reste du Fezzan. Les propriétaires privilégient donc l'accessibilité, choix logique pour des cultures orientées vers la demande locale et celle des foyers de peuplement littoraux. Les autorités publiques avaient fait de même en installant plusieurs périmètres allotis, initiant les formes individuelles de mise en valeur. Plus que dans le Wadi, le décalage entre la surface occupée par les palmeraies et celle des nouveaux jardins est spectaculaire, à la hauteur du vaste marché de consommation urbain que constitue la ville. 
La ceinture agricole se double ici de plusieurs marchés et de nombreux enclos à bétail aux portes de la ville, jusqu'à 10000 ovins et 5000 dromadaires. Ceux-ci appartiennent aux citadins qui les font garder en périphérie de la ville mais Sebha est surtout un marché saharien où sont acheminés des troupeaux élevés au Niger et au Tchad à destination des marchés de consommation urbains de l'ensemble de la Libye.

\section{LES COMPLÉMENTARITÉS ENTRE L'URBAIN ET LE RURAL}

L'étude des dynamiques agricoles du Fezzan laisse entrevoir de nombreux liens avec le processus d'urbanisation. Elle dément ainsi l'idée d'un déclin des espaces ruraux au profit de l'urbain, l'un et l'autre étant en crise avant l'intervention avant l'intervention massive de l'Etat. En revanche, leur rénovation en parallèle contribue à susciter de nouveaux liens fondés sur la complémentarité plus que sur l'opposition.

Cette complémentarité s'exprime d'abord dans les rythmes de croissance. Dans les cas de Sebha et de Brak, les villes créent leurs campagnes au fur et à mesure de leur extension. Les modalités de cette dynamique diffèrent sensiblement mais elles répondent à la nécessité de fournir des produits agricoles aux citadins et de se situer près des axes de communication majeurs. A Al Gharayfa, la dynamique est différente puisque la principale zone agricole du Fezzan est aussi la principale aire de micro-urbanisation. Les villages sont extrêmement proches les uns des autres et connaissent tous des rythmes de croissance soutenus depuis une trentaine d'années. Certes, celle-ci doit plus à l'intervention de l'Etat en terme d'emplois tertiaires et d'équipements. Mais c'est justement cette amélioration des conditions de vie et cette incitation à rester dans leur village d'origine qui encouragent les habitants à chercher dans les nouvelles formes d'agriculture des compléments de revenus, voire des placements rentables pour les plus aisés.

Cette complémentarité se retrouve dans l'évolution des pratiques spatiales. Les familles de Sebha qui possèdent des propriétés importantes s'y rendent durant l'été, y installent des jeux pour leurs enfants et des résidences secondaires. Le jardin devient donc le prolongement de l'espace privé selon un mouvement très marqué à Tripoli.

Enfin, la complémentarité s'exprime à travers les hiérarchisations sociales en cours. Jean Lethielleux notait en 1948 «qu'il faut déjà être riche pour pouvoir faire un nouveau jardin (et que) les riches n'hésitent pas à acquérir ou à créer des jardins dans les meilleures régions de culture ». Ce principe est toujours actif, particulièrement dans le cas des périmètres 
allotis de mise en valeur qui connaissent deux types d'évolutions : la sous-exploitation par manque de moyens (nous relevions que la superficie allotie était souvent trop importante par rapport aux moyens de leurs propriétaires) ou un dynamisme identique à celui des parcelles de mise en valeur individuelle. Dans ce dernier cas, ce sont les capacités d'insertion au marché et donc de choix des cultures les plus rentables qui font la différence. Les exploitations les plus dynamiques, quel que soit leur statut juridique, se caractérisent par leur superficie importante (de 10 à 40 hectares). Elles appartiennent à des familles qui disposent de réseaux de relation diversifiés et peuvent mobiliser les capitaux nécessaires. 


\section{AGIR SUR L'ESPACE À L'HEURE DE L'INFITAH LIBYENNE}

Les modalités selon lesquelles l'Etat libyen se désengage des objectifs économiques, sociaux et spatiaux qu'il s'était lui-même assigné restent difficiles à cerner et par conséquent à appréhender de manière globale. D'évidence, malgré quelques mesures spectaculaires et de nombreux discours, l'Infitah libyenne n'a pas été à la hauteur des promesses des gouvernants. En effet, les dirigeants ont rapidement été forcés de préserver les éléments sur lesquels se fonde leur légitimité alors que les leviers économiques sur lesquels ils se sont toujours appuyés, ceux de la rente des hydrocarbures, se sont progressivement érodés. Afin de résoudre ces paradoxes, le pouvoir central a privilégié deux options, plus «discrètes » mais dont on prend à peine la mesure : il a procédé à une redistribution des pouvoirs vers le niveau local et laisser se développer une économie, à dominante informelle, laissant à chacun le soin de trouver les moyens de compléter des revenus amputés par une inflation élevée et durable et la moindre redistribution de la rente.

On devine la manière dont les disparités sociales peuvent s'accroître dans le système qui se met en place. Observer quelques-uns des ressorts de l'économie, les agglomérations et l'espace rural conduit ainsi à pointer les principaux mécanismes et les indices de la mutation qui s'opère depuis la fin des années 1980 alors que l'intervention étatique était encore largement prépondérante.

La redistribution des pouvoirs s'est concrétisée par un «dégonflement» rapide et spectaculaire de l'appareil d'Etat mais les intentions des dirigeants libyens vont d'évidence au-delà des apparences. Cette déconcentration massive des moyens a profité à de nouveaux acteurs, régionaux et locaux, peu visibles pour l'observateur extérieur. Les relations entre l'Etat et les élites locales se placent donc sous le signe du renouvellement des modes de transactions entre les différents échelons de pouvoir. Celles qui lient l'Etat et les tribus perd progressivement de sa substance sous sa forme initiale mais en revanche, le soutien du régime aux élites semble aller dans le sens d'une nouvelle répartition du pouvoir. Ces dernières consolident leur place dans les rouages politiques et économiques locaux et, en retour, garantissent leur soutien au régime en place.

Il n'est donc pas étonnant que ce soit au sein des familles, et de manière plus large en s'appuyant sur les réseaux lignagers, que la redistribution des pouvoirs s'opère, certains gèrent les entreprises, les autres assurent des 
fonctions administratives et politiques. Les notables villageois s'associent ainsi plus étroitement aux rouages de l'Etat, à la fois bien intégrés dans les réseaux du pouvoir sur place et à Tripoli. Ces notables participent à l'évolution de l'espace urbain à titre privé grâce à des moyens financiers qui leur permettent d'organiser des stratégies résidentielles familiales à l'intérieur des villages et d'agir comme de véritables promoteurs immobiliers.

Grâce à leurs responsabilités locales, ils peuvent aussi exprimer une vision de l'espace éloignée des préoccupations immédiates des villageois. Ainsi, les aménagements touristiques tels que les auberges de jeunesse ou les hôtels, construits sur fonds publics, sont les manifestations du désir de certains de ces responsables de susciter de nouvelles activités mais aussi d'en être les principaux initiateurs. Ces quelques exemples montrent à quel point la séparation entre acteurs publics et privés est à manier avec précaution. Une même personne -ou famille- assume les deux fonctions mais dans les deux cas, c'est une même vision de l'espace qui s'exprime.

La fragmentation des tissus urbains traduit assez finement les disparités croissantes qui se dessinent au sein de la société libyenne, disparités de niveau de vie entre Libyens ou selon le clivage national/étranger vis-à-vis des immigrés. L'habitat, avec l'extension des formes résidentielles et non-réglementaires est un observatoire privilégié de ces processus de hiérarchisation. Mais s'il traduit une forme de crise sociale, marquée notamment par l'appauvrissement des couches modestes, jusque-là tributaires des subsides de l'Etat et la marginalité des migrants dont l'installation dans le Fezzan tend à se pérenniser, l'évolution de l'habitat prouve aussi que les habitants deviennent de plus en plus les acteurs de la transformation de l'espace dans lequel elles vivent.

La mutation des espaces ruraux du Fezzan va dans le même sens que celle des espaces urbains. Cela ne peut pas surprendre puisque les campagnes sont de plus en plus la projection de logiques et de demandes impulsées depuis les villes, tant pour la consommation que les investissements et les pratiques d'une société très largement urbanisée.

Nous disposons donc d'un fil directeur permettant de pousser plus avant le décryptage des dynamiques spatiales au prisme du changement social, en posant la question de l'émergence de l'urbanité dans le Fezzan, c'est-à-dire les modalités selon lesquelles les habitants s'approprient et territorialisent un espace durablement marqué par l'emprise de l'Etat. 


\section{TROISIÈME PARTIE}

\section{VERS UNE URBANITÉ SAHARIENNE}

Evoquer l'urbanité c'est tenter d'appréhender plus directement la dynamique sociétale qui émerge dans le Sahara libyen. Au préalable, il paraît toutefois nécessaire de revenir sur la définition de cette notion. Soulignons une des ambiguïtés liées à l'utilisation du terme d'urbanité : parle-t-on des hommes ou des lieux qu'ils habitent ? Cette ambiguïté vient sans doute du fait que l'urbanité a droit de cité et valeur de notion dans d'autres sciences sociales, par exemple la sociologie. Le fait que les réflexions autour de l'urbanité se développent est révélateur de la volonté des géographes de dépasser les limites de la notion d'urbanisation et le flou que laisse planer celle d'urbain.

Pour Roger Brunet (1993), l'urbanité est le «caractère de ce qui est urbain », c'est-à-dire par extension et en tant que synonyme de civilité l' «ensemble de traits de comportements positifs, impliquant courtoisie, respect de l'autre, bonnes mœurs et usages que l'on assurait être le propre du citadin ». L'emploi de l'imparfait et les expressions utilisées ne sont pas anodins. L'urbanité dont parle Roger Brunet avec ironie renvoie à une vision condescendante, celle du « citadin de souche », vis-à-vis du monde rural et de la « ruralisation des villes »; une vision connotée donc, qui dans ces conditions conduirait à proscrire le terme de toute analyse scientifique. Pour donner sens aux mutations de l'urbain dans les pays industrialisés, Jacques Lévy (1994) part du constat que la production dans la ville a plus été étudiée que la production de la ville. Dans ces conditions, «l'urbanité étudie les effets productifs (de la ville) sur l'ensemble de la société urbaine... Elle est une force de production économique, sociologique, politique, spatiale, temporelle, individuelle » où le spatial se réduit à une entrée parmi d'autres. En 1986, lorsque Pierre Signoles mentionne le terme d'urbanité dans le titre d'une synthèse, c'est pour l'assimiler à celui de citadinité, sans plus de précision. En revanche, dix ans après, dans un ouvrage collectif coordonné par le même auteur, Rachid Sidi Boumedine (1996) considère que « lorsque nous parlons d'urbanité, nous sommes en terrain sûr. Les vieux débats sur la ville, sur sa caractérisation... reposent sur un consensus quant à la prise en compte des caractères essentiels du fait urbain. Et ce consensus repose sur une série de données qui ont en commun 
d'être observables, mesurables, comparables... L'urbanité, c'est (être dans la ville)».

La présentation de ces trois définitions risque d'obscurcir le débat tant elles différent. Pour Rachid Sidi Boumedine (1996), l'élément central n'est plus la ville mais la personne. Le terme d'urbanité relève pour lui du jargon disciplinaire classique et ne mérite pas d'être plus développé. Ceci s'explique sans doute en partie par le fait que son propos, dans l'article cité, est plutôt axé sur la citadinité. Ce qu'il remet sans doute en cause, ce n'est pas la pertinence du thème mais le biais utilisé pour l'aborder. En revanche, la définition de Jacques Lévy semble compléter et « rafraîchir » celle de Roger Brunet dans le sens où dans les deux cas, il est question de ce que diffuse la ville. Sauf qu'à la vision restrictive et passéiste, celle de l'acculturation partie des cités et des citadins vers les campagnes et leurs habitants, succède une vision de la ville comme centre d'impulsion des dynamiques contemporaines vers d'autres espaces. La ville n'est plus seulement un centre de concentration et de diffusion des fonctions économiques, culturelles et de commandement sur les campagnes qui l'entourent mais un objet sociétal.

Dans cette optique, on peut considérer que dans le Fezzan l'intervention étatique a essentiellement contribué à créer des agglomérations, simples lieux de concentration des populations et des services. A tous les niveaux de la hiérarchie urbaine, cette action sectorielle et fonctionnelle s'est manifestée avec force à travers la mise en œuvre d'une planification urbaine, la définition d'un appareil législatif et la production de plans d'aménagement qui ont guidé les étapes de l'urbanisation d'Etat.

Cette vision de la ville s'est heurtée à des impératifs : il fallait agir dans l'urgence, avec des moyens importants et donc déléguer à des bureaux d'étude extérieurs la conception et la construction des agglomérations. Ces dernières connaissent des dérèglements depuis que l'érosion de la rente pétrolière ne permet plus d'assurer la maintenance que requièrent ces modèles urbains importés, inadaptés aux conditions climatiques et à la demande sociale des populations. Mais dans quelle mesure le modèle pensé et conçu par le haut est-il approprié par ceux qui l'habitent ? L'émergence de nouveaux modèles permet de pointer la diversité de «l'habiter » dans le Fezzan.

Cette lecture de l'habitat ne donne pas à voir le fonctionnement social des lieux, la manière dont se produisent les sociétés urbaines que nous désignons ici par le terme d'urbanité. Deux modes d'urbanités sont en gestation, révélateurs de tendances que l'on retrouve dans tout le Sahara 
maghrébin. D'une part, à l'image de Sebha, de véritables mosaïques urbaines se dessinent, dans des villes-charnières, villes-carrefours, qui se remodèlent socialement à l'épreuve des flux migratoires qu'elles captent. D'autre part, les réseaux villageois oasiens, tel celui du Wadi Al Haya, permettent de pointer de nouvelles expressions d'urbanité, lesquelles paraissent émerger en faisant l'économie du support matériel de l'agglomération, bref, une urbanité sans ville. 


\section{CHAPITRE 7 - DE LA VILLE PENSÉE À LA VILLE VÉCUE}

Afin de prendre la mesure de la dimension sociétale des agglomérations, il nous semble nécessaire de déplacer l'analyse vers l'amont et l'aval du processus d'urbanisation étatique. Par delà la simple appréhension des conséquences spatiales, il est donc tentant de déceler les intentions et les choix qui ont précédé les plans d'aménagement urbain, ceux des dirigeants qui élaborent un projet puis ceux des aménageurs qui tentent de donner corps à ce projet à partir des informations dont ils disposent. Il y a d'abord cette montée en puissance de l'Etat comme acteur prépondérant qui exprime sa vision du territoire mais aussi sa vision de la société à travers une planification à l'échelon national. Ces visions se heurtent à des problèmes multiples, dépendance technique vis-à-vis de bureaux de planification étrangers, difficulté à estimer et à projeter les rythmes de croissance et les besoins des populations.

L'invention d'un modèle urbain étatique par les aménageurs, forcément incomplet, s'accompagne de mouvements d'adaptation, d'appropriation et de contournement. Les agglomérations connaissent des transformations par rapport aux intentions de ceux qui les ont pensées et conçues par le simple fait qu'elles sont désormais habitées. Ce mouvement se perçoit à travers l'habitat mais aussi à l'analyse des dynamiques du centre des îlots d'habitat, à la fois vides dans la ville et objets de réappropriation. Plus problématiques sont les distorsions entre le modèle et sa traduction spatiale que la multiplication des dysfonctionnements dans le tissu urbain révèle, à la fois signes de crise mais peut-être aussi de mutation.

\section{LA CONCEPTION D'UN MODÈLE D'URBANISATION}

Dès les années 1950 et 1960, l'Etat libyen issu des indépendances s'est doté dans le champ de l'urbain d'un appareil législatif et d'intervention de grande ampleur car très vite la planification de l'urbain devient une nécessité dans l'ensemble du pays. Elle se fonde d'abord sur l'urgence ; il faut résoudre les problèmes liés à l'exode rural, à la pénurie de logements et à la faible articulation entre les principaux de foyers de peuplement de Tripolitaine, Cyrénaïque et du Fezzan. La planification exprime aussi, plus nettement après la prise du pouvoir par Mouammar Kadhafi en 1969, la volonté des autorités de réguler de manière exclusive l'aménagement du territoire national. Un bref retour sur la mise en place des politiques 
d'intervention dans le champ de l'urbain apporte un éclairage sur l'émergence du modèle urbain étatique.

Durant les années 1960 et 1970, une planification et un appareil législatif de régulation et d'accompagnement sont progressivement mis en place, analysés par Borham Attalah et Mona Fikry (1972). Il en ressort deux points essentiels : «l'élaboration de ces plans... est essentiellement l'œuvre des bureaux de l'administration centrale, souvent composés de spécialistes étrangers ». En revanche, si pour les principales agglomérations urbaines, "tous les détails d'aménagement technique, économique et démographique » furent préparés, pour les plus petites, seules « les grandes lignes de l'aménagement » furent envisagées.

Ces décisions, prises à l'époque de la royauté (1951-1969), ont des conséquences qui perdurent après la chute du régime sous l'impulsion de Doxiades Associates et de Whiting Associates International, «deux organismes de conseillers internationaux ont pris une part active dans l'élaboration de la politique libyenne de l'urbanisme ». Or leurs études privilégient les agglomérations principales, Benghazi et Tripoli, dont la croissance s'accélère, nourrie des flux de retour des Libyens expatriés durant la colonisation italienne et de l'exode rural alors que c'est l'ensemble du territoire libyen qui connaît de nombreuses mutations liées à la croissance démographique accélérée et aux flux migratoires internes en direction des villes petites et moyennes du territoire. Ces dernières se transforment donc pour l'essentiel en marge des «grandes lignes de l'aménagement » trop sommaires.

Le décalage entre l'espace planifié à l'horizon 1988 et l'espace réellement urbanisé en 1978 est révélateur de la difficulté qu'éprouvent alors les autorités libyennes à construire une politique nationale d'aménagement urbain. Il est frappant de voir à quel point l'extension spatiale des agglomérations sahariennes fut sous-estimée, leur taux de croissance attendu étant nettement inférieur à leur taux de croissance réel sur la période. Les planificateurs prévoyaient en effet une croissance continue de la population sur le littoral, nourrie par la poursuite du mouvement d'exode du Sud vers le Nord. Le cas de Jalou, petit centre saharien dans la principale région d'exploitation pétrolière, au sud de Benghazi (S. Qezeiri et R. Lawless, 1986), révèle les décalages entre la politique d'intégration nationale du Sahara et celle des petites agglomérations. Alors que le plan prévoyait une baisse significative de la croissance démographique de Jalou entre 1968 et 1988, la population a presque doublé. Or, le petit centre supposé isolé allait bénéficier à la fois du développement d'un périmètre agricole étatique, de sa situation le long 
de l'axe routier Ajdabiya-Koufra et de sa promotion administrative au rang de chef-lieu de Baladiya. Dans ces conditions, le mouvement d'exode s'inverse et provoque une poussée urbaine soutenue. De même, Al Jawf, principal village du groupe d'oasis de Koufra, connaît sous l'impulsion des pouvoirs publics et de la guerre du Tchad un afflux massif de population, nomades, réfugiés, militaires, techniciens, qui provoque un essor inattendu de l'agglomération, en état de léthargie depuis son occupation par les Italiens en 1931.

La généralisation de la croissance urbaine en Libye et la poussée des petites villes qui en a résultée n'est sans doute pas étrangère à l'évolution du rôle de l'Etat dans le secteur du bâtiment durant la royauté. L'appareil juridique était destiné à juguler un marché de la construction d'initiative privée. Mais rapidement, « l'Etat libyen... ne s'est pas limité à édicter des normes d'urbanisme et à lutter contre la spéculation... il chercha à devenir aussi le grand constructeur de logements pour les distribuer aux citoyens » (B. Attalah, M. Fikry, 1972). Ce rôle central s'accroît durant les années 1980 puisque l'Etat devient dans le domaine de l'habitat «financier, régulateur, spéculateur, administrateur, constructeur, propriétaire... » (S. Qezeiri, 1989). En multipliant ses domaines d'intervention, son rôle évolue du strict domaine de la régulation de la construction à celle de la planification à l'échelle de toute la Libye. C'est dans ce cadre que sont élaborés de nouveaux plans d'aménagement.

Progressivement, les petites agglomérations sont prises en compte par la planification. Durant la seconde moitié des années 1970 et le début des années 1980, de nouvelles études, plus détaillées, sont à nouveau confiées à des cabinets d'experts internationaux. La plus importante, menée par Italconsult, est une étude exhaustive du peuplement en Libye publiée en 1976. La préoccupation essentielle devient celle de l'équilibre du système urbain à l'échelle du territoire national. Les rédacteurs de l'étude proposent donc de recomposer la hiérarchie urbaine, afin d'inciter au rééquilibrage de la population sur le territoire national. Ces analyses, reprises dans le cadre d'un plan d'aménagement national marquent le véritable lancement de la politique de multiplication des petites et moyennes agglomérations sur l'ensemble du territoire, sur la période 1981-2000. Elle est complétée par les études de Doxiades (1979), Polservice Consulting Office (1980), Finnmap et Speerplan (1981) sur les différentes régions du pays. Ces études prennent appui sur une couverture aérienne exhaustive et la cartographie de toutes les agglomérations, y compris des plus petits villages, est établie à l'échelle du $1 / 1000^{\text {ème }}$ (cadastre) et du $1 / 5000^{\mathrm{eme}}$ au $1 / 15000^{\text {ème }}$ (planification des nouveaux équipements et de l'habitat). La nécessité d'agir dans l'urgence conduit à nouveau les responsables de 
l'aménagement à dépendre d'experts qui mènent des études générales peu en rapport avec les réalités locales afin de juguler une urbanisation effectuée hors des plans précédents. En outre, l'exécution des travaux est confiée à des sociétés étrangères de bâtiments et travaux publics selon le principe des projets « clés en main ». La réponse quantitative à la demande de logements est donc en grande partie comblée au prix de l'imposition d'un modèle standard et allogène d'urbanisation.

\section{CRISE ET RÉAPPROPRIATION DU MODÈLE IMPORTÉ}

L'une des constantes des agglomérations du Sahara libyen est leur état sanitaire critique. Partout, les signes de crise sont flagrants : des ordures jonchent le sol en tas de superficies variables, d'immenses flaques d'eau stagnent, y compris dans les plus petits villages. Crise de croissance ou crise de fonctionnement du modèle étatique ?

L'usage des « vides urbains » constitue l'une des clés de lecture de ces dysfonctionnements, mais aussi des régulations que les habitants mettent en œuvre pour y pallier. Les terrains vagues sont nombreux dans les agglomérations au point de faire parti du paysage et d'acquérir un rôle dans les dynamiques de réappropriation du tissu urbain. La comparaison du bourg d'Al Gharayfa à près de 20 ans de différence renseigne sur les modalités de la création des terrains vagues.

Durant la période de construction des logements et des infrastructures, les aménageurs ont privilégié la « dé-densification » du bâti, rompant ainsi avec l'organisation des ksour. Les premiers ensembles étaient éloignés les uns des autres, reliés par un quadrillage de pistes, parallèles et perpendiculaires à la route. L'extensivité du bâti a dans un premier temps conduit à l'éparpillement des immeubles, bâtiments administratifs et résidences individuelles. Les constructions furent implantées le long de la route et des pistes. Le décalage entre le nombre de bâtisses et la superficie de l'espace à urbaniser a donc entraîné la multiplication des vides, le centre des îlots urbains restant vide, en contraste avec les axes. C'est là l'indice, en somme, du décalage entre les intentions des planificateurs et les choix des habitants.

C'est à l'initiative de ces derniers que cette situation change rapidement. Le comblement des îlots s'accompagne en effet de la diversification du bâti, le jardin du centre de l'îlot disparaît, un lotissement résidentiel d'Etat est construit au sud, ainsi que quelques résidences privées. Surtout, l'emprise de l'habitat non réglementaire s'étend puisque l'on relève quatre sites différents : l'un se résume à un enclos en parpaings 
et à une boutique, un second à des résidences individuelles, le troisième est loué à des étudiants et le dernier se greffe sur les ruines d'un des vieux sites villageois. Sur un périmètre réduit, on trouve l'essentiel des formes d'habitat présentes dans les villages du Sud libyen et on devine aussi quelques-uns des ressorts qui ont conduit à leur construction. Si l'enclos correspond à la volonté de son propriétaire de compléter ses revenus par l'élevage d'ovins et une épicerie, les autres cas révèlent à quel point l'accès à la propriété pose problème : des particuliers aspirent à posséder une résidence individuelle mais ne peuvent accéder aux normes de l'habitat réglementaire ; des étudiants pâtissent de la crise du logement universitaire à Oubari et s'installent dans une baraque louée à 20 kilomètres de l'Université ; la plupart des immigrés subsahariens n'ont généralement pas d'autre choix que de s'installer dans les interstices de l'espace urbain. Le comblement de l'îlot ne se réduit donc pas seulement à une dynamique spatiale, il reflète aussi un changement social.

L'assainissement est l'un des principaux problèmes posés par la rapidité de la croissance urbaine. Quatre systèmes d'évacuation sont utilisés dans les petites agglomérations libyennes, les puits perdus (dans les vieux centres), les fosses étanches, «vidangées à la demande des municipalités », les fosses communales... «dans les nouveaux lotissements construits par l'Etat... pour un groupe de dix habitations » et finalement, le réseau collecteur relié à une station d'épuration dont seules parmi les petites ville Al Marj et Al Khoms étaient dotées au milieu des années 1980 (S. Qezeiri, 1986). Dans la mesure où les agglomérations du Sud ont été construites récemment par l'Etat, c'est le système des fosses septiques communales qui prédomine. A défaut d'un réseau complet d'évacuation des eaux usées, les îlots avaient été dotés de fosses communes, censées être vidées régulièrement par les services d'assainissement installés dans chaque agglomération. Or, plus on monte dans la hiérarchie des agglomérations, plus les flaques sont nombreuses et étendues, devenant parfois de véritables égouts à ciel ouvert, en particulier à Oubari (photo 3).

Cyniques signes de la rapidité du processus d'urbanisation, ces flaques témoignent de la croissance rapide de la consommation en eau par les particuliers. On touche là à un paradoxe qui concerne la plupart des espaces sahariens : si les forages et les motopompes permettent de pourvoir à la demande en eau des populations des agglomérations, en revanche la nécessaire mise en place d'un réseau d'évacuation s'avérerait coûteuse. Les dirigeants des services d'urbanisme en repoussent donc l'échéance d'un plan à l'autre, par faute de moyens financiers suffisants. Les forages se multiplient, à l'intérieur même des quartiers, comme à Sebha, 
contribuant ainsi à repousser à plus tard les risques de pénurie. La consommation croît, en même temps que le gaspillage, rendant ainsi le problème de l'assainissement de plus en plus critique.

Photographie 3 : « Lac» d'eau stagnante à Oubari

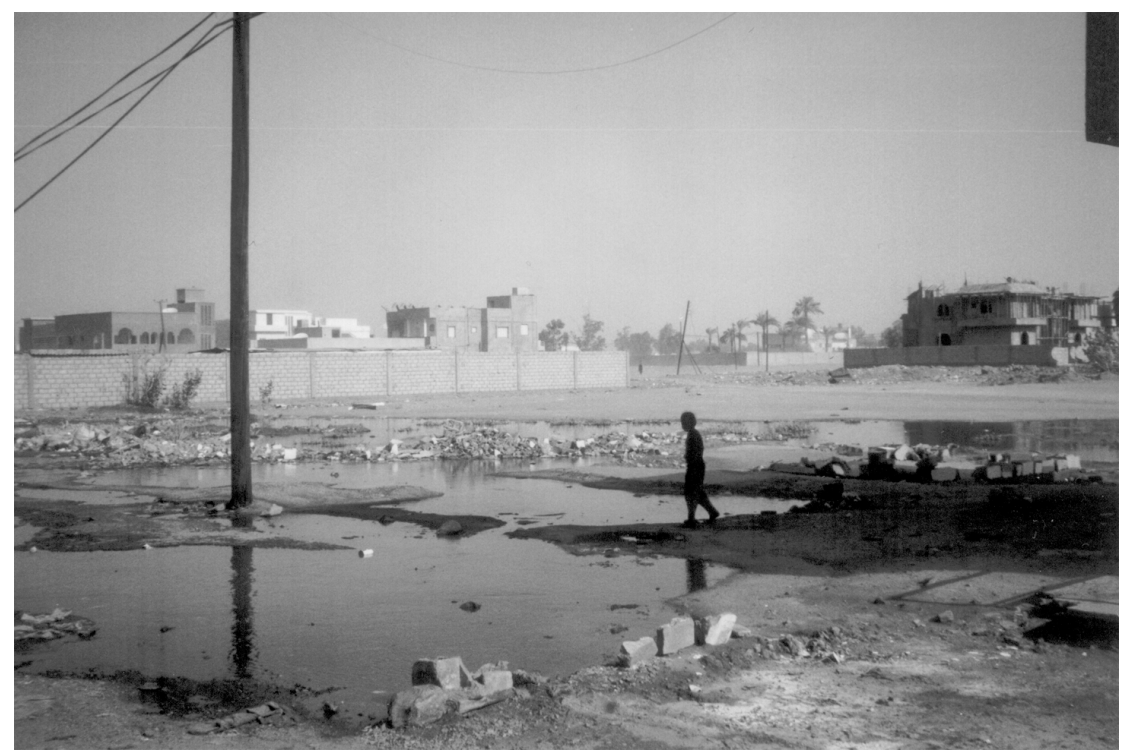

Face à ce changement, les pouvoirs publics sont démunis comme en témoignent les petits camions-citernes qui arpentent les rues d'Al Gharayfa afin de vider fosses et flaques. Leurs chauffeurs déversent ensuite leur contenu en périphérie de l'agglomération où de véritables lacs se forment en cas de ruptures de canalisations... jusqu'à ce que les températures estivales ne les assèchent. A Oubari, il est bien question de mettre en place un réseau complet d'évacuation mais celui-ci était déjà prévu dans le plan d'aménagement précédent. Au retard pris, s'ajoute le problème du recyclage des eaux... à moins de déplacer le problème du centre vers la périphérie urbaine comme à Sebha, dotée d'une station d'épuration mais dont les eaux sont ensuite déversées dans un lac.

Seules les zones d'habitat informel échappent encore au problème d'assainissement car elles ne bénéficient pas du système d'approvisionnement municipal. Leurs habitants creusent eux-mêmes des fosses à proximité des habitations et se cotisent pour les faire vider régulièrement. Mais il est probable que l'amélioration des conditions de 
vie entraîne une consommation d'eau croissante et donc à terme les mêmes problèmes d'assainissement.

Le problème des déchets est commun à toutes les agglomérations. A Takarkiba, on peut dénombrer au moins six tas d'ordures conséquents à l'intérieur d'un village d'environ 700 habitants. Leur répartition obéit à une logique de proximité puisque les petits dépôts se situent dans les espaces vacants au sein des îlots d'habitat constitués par l'amoncellement des déchets jetés par les riverains. Les tas d'ordures les plus conséquents se situent plutôt en périphérie de Takarkiba, vers la palmeraie, notamment dans le vieux village qui se transforme en décharge et les fondements des nouveaux lotissements, parsemés d'ordures regroupées en tas. L'extension du bâti repousse les limites du village et à chaque nouvelle construction les ordures périphériques sont repoussées à la pelleteuse plus avant dans la palmeraie.

Cette tendance se confirme à $\mathrm{Al}$ Gharayfa où les ordures se trouvent dans le centre des îlots les moins bâtis et en périphérie, le long des axes goudronnés. Toutefois, l'importance des déchets est ici telle que la mise en place d'une décharge à la sortie de la ville est désormais indispensable. Néanmoins, comme pour les eaux usées, le décalage entre les quantités à déplacer et la modestie des moyens laisse douter de la durabilité de ces solutions. Malgré cette crise généralisée de la gestion collective des déchets, certains quartiers résidentiels de Sebha sont dénués de tous tas d'ordures, laissant présumer que, comme à Tripoli, des systèmes privés de ramassage se mettent en place.

L'effet de taille déjà prégnant à propos de la question des eaux usées contribue à un clivage entre Sebha et les autres agglomérations. La taille de Sebha impose une réponse de la part des pouvoirs publics, tant du point de vue de l'assainissement que dans d'autres domaines de la régulation de l'urbain. A bien des égards, cette réponse paraît modeste mais l'importance et la durée des taux de croissance hypothéquaient jusqu'à présent toute possibilité d'adéquation entre les besoins et les services disponibles. A défaut de pouvoir anticiper, les autorités tentent au moins de combler le retard.

Plus préoccupante est la situation des petites agglomérations. Leur taille, et sans doute aussi les modestes besoins des habitants à l'époque de la construction des villages, expliquent qu'il a pu être possible de faire dans un premier temps l'économie d'une gestion collective de l'assainissement. Depuis, les taux soutenus de croissance démographique provoquent une crise qui progresse avec l'urbanisation, laissant augurer de problèmes qui vont se poser avec acuité dans un proche avenir. 


\section{LE LOGEMENT, INDICE DE CHANGEMENT SOCIAL}

Généralement, le logement porte les premières marques d'une appropriation de l'espace par les individus. Cette tendance est d'autant plus marquée dans le Fezzan que le fait de bénéficier de logements identiques a parfois conduit leurs propriétaires à effectuer les mêmes types de transformation. Pourtant, la diversité des formes d'habitat et par conséquent des modalités de leur réappropriation nous dit beaucoup sur les sociétés du Sahara libyen.

Au-delà de la recherche d'un hypothétique modèle saharien ou de l'énumération et de la description de tous les types d'habitat, il semble donc plus opportun de dégager une grille de lecture des «figures de l'habiter », à même de nous permettre de progresser dans une lecture du changement social. Ces figures seraient au nombre de trois (J. Lévy, 1994), la nécessité étant la forme la plus élémentaire, le pouvoir-habiter, qui signifie que les individus peuvent élaborer des choix et le vouloir-habiter lorsqu'ils sont en mesure d'arbitrer les choix qui s'offrent à eux.

\section{Photographie 4 : Transformation des logements collectifs public à Al Gharayfa}

Dans l'exemple ci-dessous, une seule famille vit dans chacun des petits logements collectifs. Comme pour les maisons individuelles, l'une des premières transformations opérées par les habitants consiste en la surélévation de la bâtisse par ajout d'un étage où logeront les enfants des propriétaires, parfois avec leur épouse s'ils se marient mais n'ont pas les moyens de faire construire.

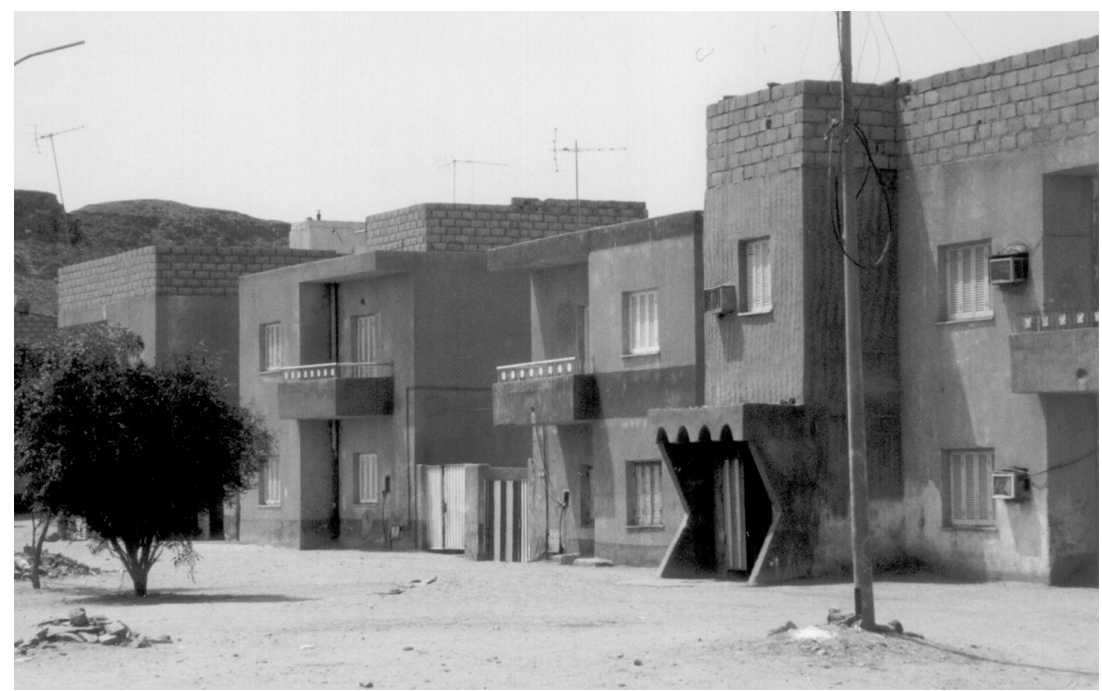


La figure dominante est celle du pouvoir-habiter, promue durant la période d'intervention étatique à travers la construction de logements pour les populations. Dans la majorité des villages, l'habitat se résume essentiellement à la maison individuelle en lotissement. Le collectif (photo.4) ne diffère pourtant pas forcément de l'individuel, sauf à Sebha, où ses dimensions sont en proportion avec la taille de la ville.

La nécessité est minoritaire par rapport au pouvoir-habiter malgré la croissance rapide de l'habitat non-réglementaire. Elle concerne essentiellement l'habitat aux conditions sanitaires les plus critiques. Les Libyens y sont moins présents que les immigrés ou les réfugiés. Plusieurs situations peuvent être distinguées. $\mathrm{La}$ première relève de l'autoconstruction, fréquente, comme nous l'avons déjà mentionné, depuis la rétraction du rôle de l'Etat dans la construction. Elle s'effectue soit ex-nihilo, dans les vides du tissu urbain (photo.5), soit à partir des ruines des ksour. La seconde relève de l'insertion des migrants dans le tissu urbain, qui s'effectue aux marges des agglomérations dans les logements délaissés par les Libyens ou bien dans leur centre (photo.6).

\section{Photographie 5 : Autoconstruction à Al Gharayfa}

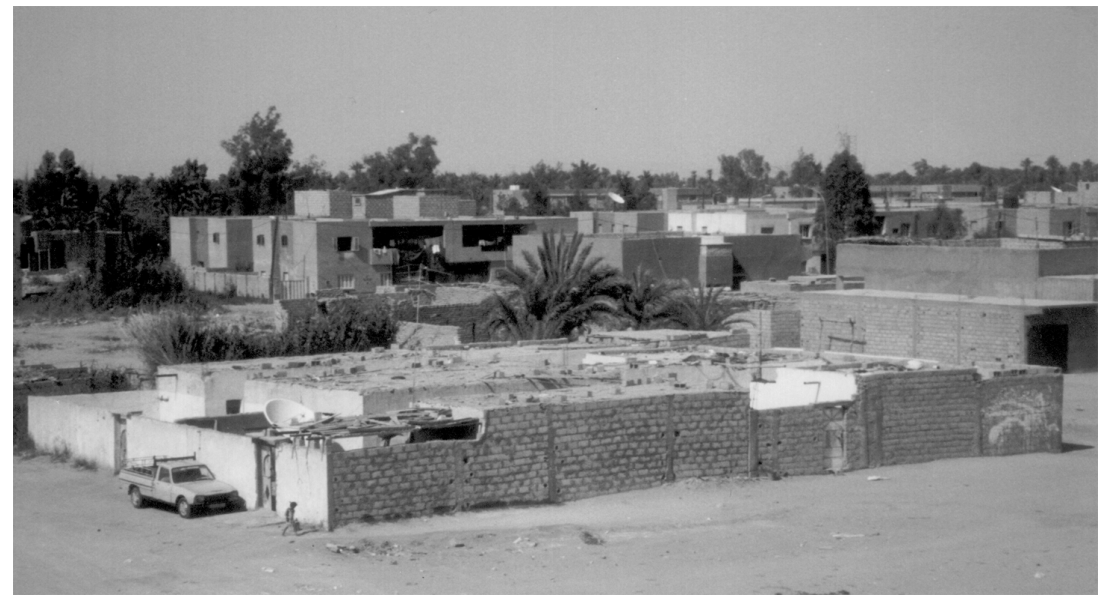

L'exemple d'Al Hajara (photo.7), à Sebha, nous amène à réfléchir à la situation du ksar dans le contexte d'urbanisation actuel. Quelques constatations d'Amina Zine (1994), complétées par nos propres 
observations conduisent à distinguer plusieurs modes d'intégration du ksar dans l'agglomération, dans l'ensemble du Sahara. La rapidité de la croissance urbaine, l'intégration dans des villes de tailles bien différentes, les choix des populations et ceux des pouvoirs publics (plan de rénovation ou d'abandon au profit de nouveaux sites), très variables selon les lieux, constituent les variables d'une typologie, même sommaire.

\section{Photographie 6 : Locatif pour migrants à Sebha}

Dans la plupart des cas, l'exiguïté des logements ou bien l'agrandissement de la famille motive la construction d'un étage supplémentaire. Il est en revanche fréquent de trouver dans les quartiers où vivent les couches aisées, à Sebha, des abris sommaires que les propriétaires ont construits afin de loger leurs employés étrangers (ici un Egyptien) et de compléter leurs revenus grâce aux loyers.

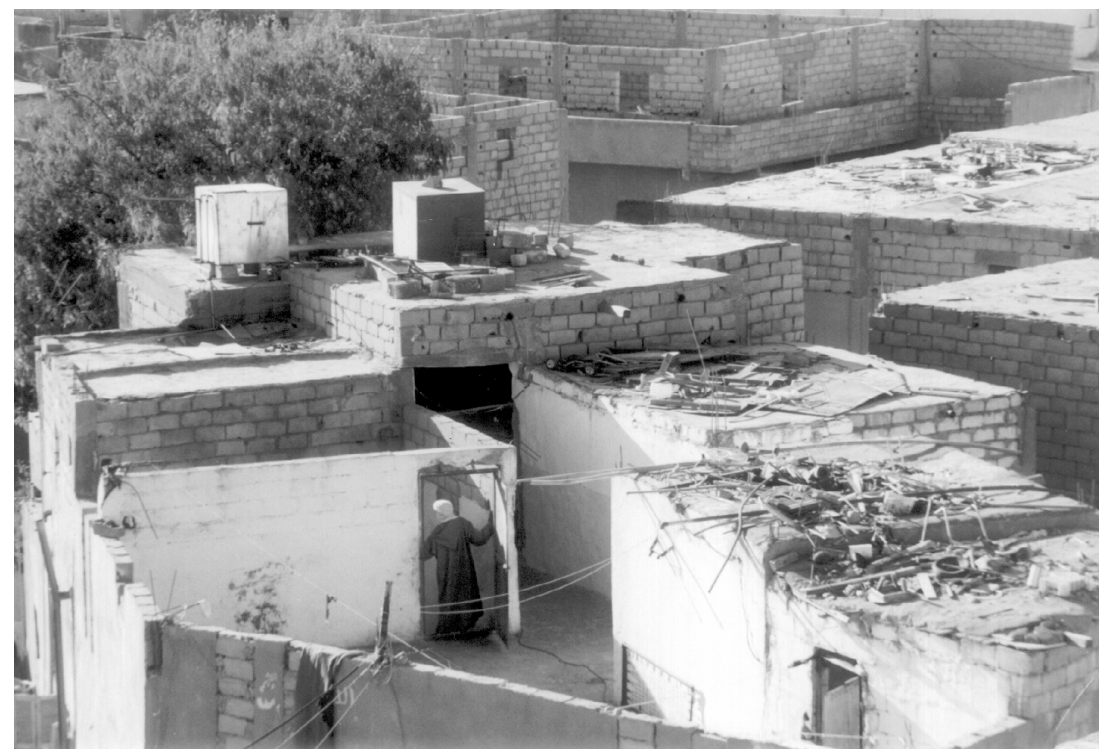

Le ksar peut toujours faire fonction de centre-ville, mais c'est alors un centre densément peuplé dans une agglomération importante. Délabré, il n'en demeure pas moins le lieu où s'installent les populations les plus modestes, comme cela a souvent été le cas depuis quelques décennies dans les médinas des métropoles du Monde arabe. Le ksar-patrimoine peut aussi se situer dans le centre ville (Tozeur, Ghadamès), à proximité du nouveau centre, commerçant et administratif. Cœur historique, il est souvent rénové car il contribue à médiatiser l'image de la ville à l'extérieur. Le ksar enclavé (Nefta) l'est à des degrés divers puisqu'il fait partie intégrante de 
l'agglomération au même titre que les quartiers plus récents. Le ksar abandonné, en ruine, est un cas très répandu de part et d'autre du Sahara. Les causes en sont diverses, mais généralement liées à l'évolution de la communauté et des représentations qu'elle se fait de l'habitat, où souvent l'ancien est déclassé au profit des constructions récentes. On ne peut évidemment pas négliger les mesures qui contribuent à une conservation ou à une rénovation liées à un classement patrimonial (Ghadamès, Ghardaïa, Chinguetti) ou à une valorisation touristique (Tamerza).

La Libye ne déroge pas à la diversité des situations que l'on retrouve dans toutes les régions sahariennes. Mais généralement la situation est plus tranchée puisque le ksar est soit abandonné soit un lieu-refuge pour les populations qui s'insèrent dans les villes, qu'ils s'agissent des réfugiés touaregs ou toubous et des migrants subsahariens.

\section{Photographie 7 : Squat Toubou dans les ruines du ksar d'Al Hajara (Sebha)}

Al Hajara est l'un des ksour qui composait l'ensemble oasien de Sebha que ses habitants ont quitté pour s'installer dans la ville moderne. Les réfugiés Toubous tchadiens venus s'installer à Sebha dans les années 1980 s'accommodèrent, comme aujourd'hui les migrants subsahariens, de ces villages en ruine. Ils louent les maisons dans lesquelles ils vivent aux propriétaires libyens et rénovent les ruines afin de les transformer en logements décents.

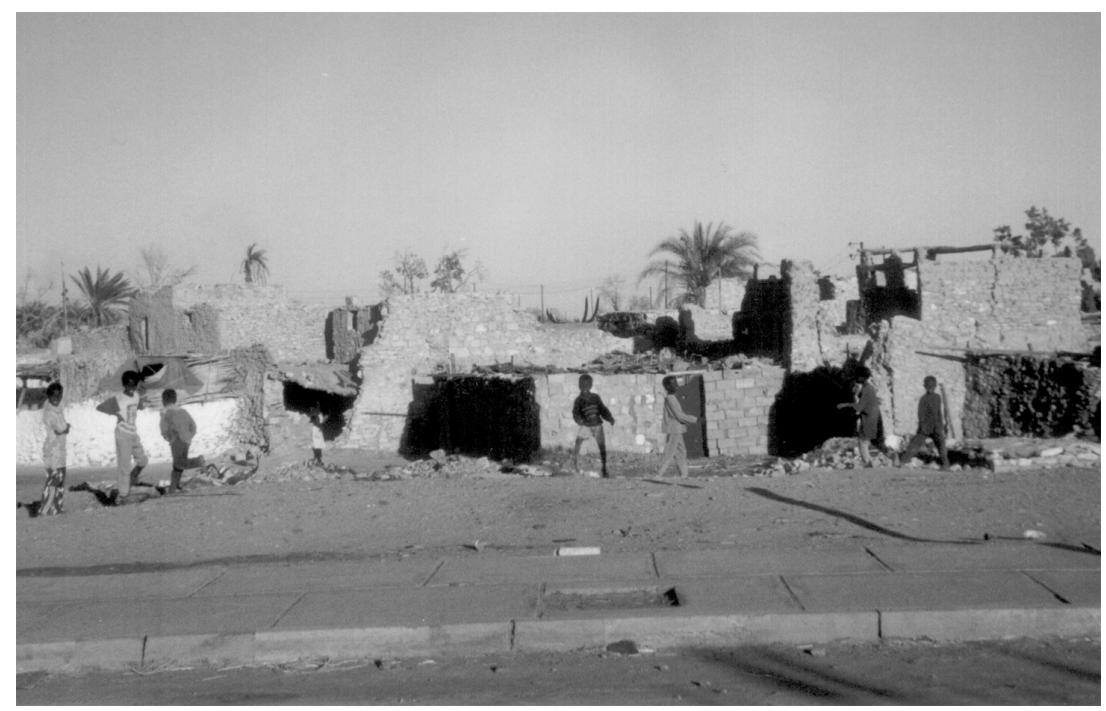


La troisième situation relevant de la figure de la nécessité, est celle des villages de fixation des nomades (photo.8). Ils sont généralement nettement séparés du tissu urbain actuel et construits suivant un schéma répandu, de la tente à l'enclos, puis de l'enclos à la maison par la construction de pièces en dur.

La diversité de l'autoconstruction ne se retrouve pas seulement dans les formes, elle relève aussi des matériaux employés. Les deux types dominants sont la brique de ciment et la brique de terre. La première est la plus employée car elle présente l'avantage d'être résistante, facile à confectionner et à acquérir puisque chaque village compte un atelier de fabrication. Autre matériau, la brique de terre crue, traditionnellement utilisée pour bâtir les ksour, ne l'est plus aujourd'hui que dans quelques sites de fixation des nomades. L'importance des ateliers de fabrication donne d'ailleurs une idée de la rapidité de la croissance du village.

\section{Photographie 8 : Nomades et réfugiés tchadiens en périphérie de Sebha}

Plusieurs milliers de réfugiés tchadiens, installés en périphérie de Sebha, vivent dans des tentes progressivement consolidées et aménagées. C'est là qu'ils participent à l'élevage et au négoce du bétail qui est consommé par les citadins ou bien transite entre Tchad, Niger et Tripolitaine.

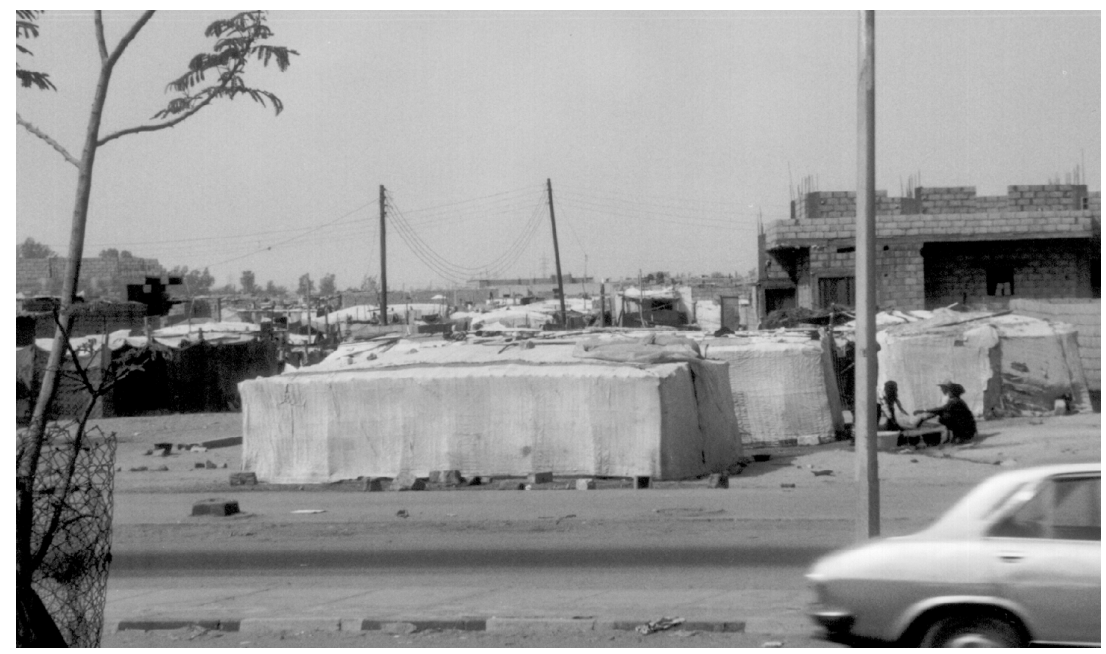

La dernière figure, le vouloir-habiter, correspond à l'habitat résidentiel privé, qui s'étend rapidement. Dans les villages et les bourgs, les choix de 
localisation sont souvent restreints par un facteur surdéterminant, celui de la proximité familiale. Mais à Sebha, le processus de résidentialisation est déjà largement engagé (fig.14).

\section{Photographie 9 : Cloisonnement des balcons à Sebha}

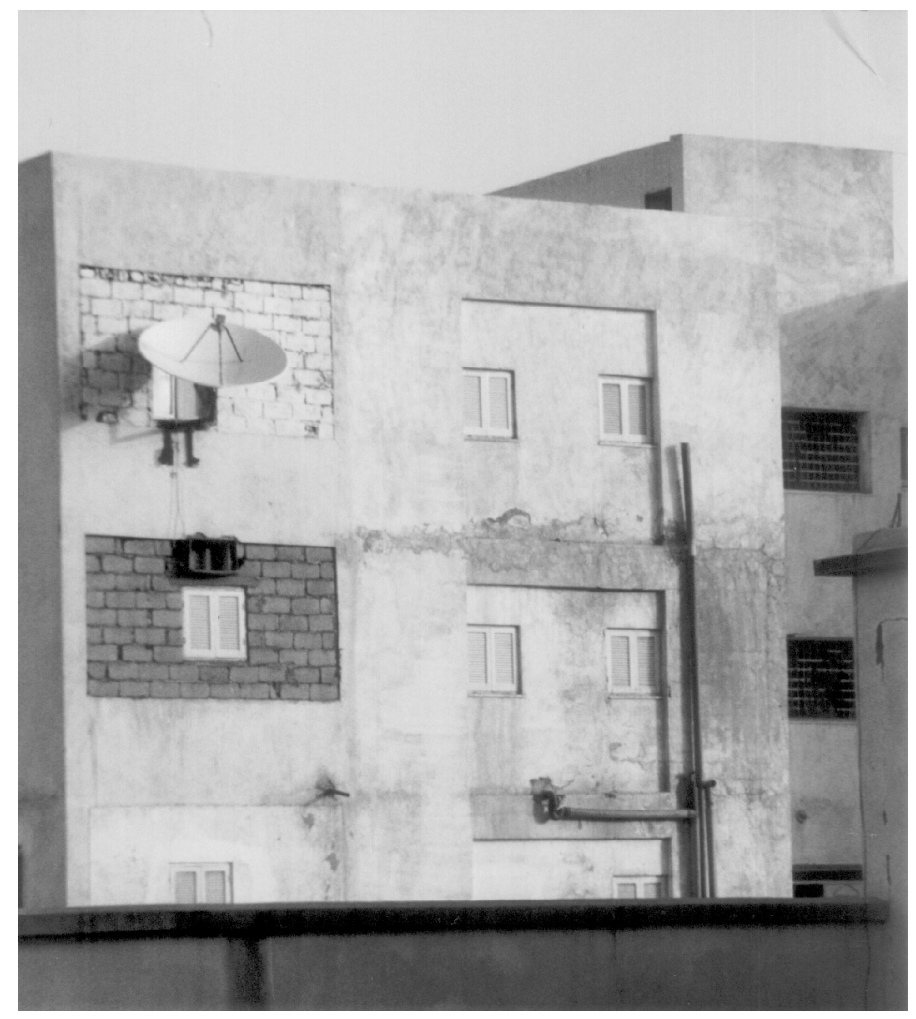

Nadir Boumaza (1993), en observant les villes maghrébines, recense trois conflits de valeurs à la traduction flagrante dans l'habitat: le conflit occidental/oriental fondé sur l'ambivalence entre l'aspect extérieur de la maison oriental et le confort intérieur selon les normes occidentales ou la diffusion du bâti type «HLM»; le conflit passé/présent, qui croise le précédent car la référence historique est toujours orientale (ouverture/fermeture de la maison); le conflit modernité/tradition caractérisé par l'opposition à la modernité qui produit parfois une volonté de retour à une tradition imaginée, magnifiée. Si les trois types de conflits sont séparés pour la clarté de l'exposé, ils ne fondent 
pas trois modèles différents mais sont entremêlés, selon l'idée qu'avance Françoise Navez-Bouchanine (1991) à partir d'études menées dans les métropoles marocaines. Il y a bien, selon elle, un modèle de l'habiter "rêvé » par les couches populaires pour lesquelles l'appropriation de l'habitat consiste en une transformation du logement visant à combler l'écart entre le lieu où l'on vit et celui où l'on aspirerait à vivre. Ce modèle, loin d'être circonscrit aux métropoles, atteste du partage de valeurs esthétiques et sociales à l'échelle d'un territoire national voire du Maghreb.

La volonté de séparer l'ouvert de l'intime est symptomatique de ces conflits, particulièrement dans les grandes villes. Dans les villages, la préservation de l'intime était prévue dans le cadre des lotissements si l'on se fie à la hauteur des murs. L'ouvert, c'est l'espace où sont accueillis les invités, l'intime est réservé à la vie familiale. Or même si les logements sont exigus, la place du premier est disproportionnée par rapport au reste de la demeure puisqu'il peut correspondre au tiers de la résidence. De manière presque systématique, les balcons sont cloisonnés (photo.8), pour des raisons pratiques, gagner une pièce de plus et se protéger du soleil mais aussi avec le souci de préserver cette intimité. Cet exemple illustre de manière flagrante le décalage entre l'importation d'un modèle et l'indispensable réappropriation à laquelle procèdent les habitants. L'exiguïté est un autre élément plus commun qui peut aussi expliquer ce cloisonnement mais dont la conséquence dominante réside dans la surélévation des résidences individuelles. En fin de compte, les éléments communs avec d'autres régions sahariennes, voire arabo-musulmanes, l'emportent sur les spécificités libyennes.

En tentant de répondre aux urgences de la croissance démographique et aux recompositions entraînées par les migrations, les planificateurs n'ont évidemment pas agi de manière neutre. Rapidement, l'Etat s'est imposé comme l'acteur essentiel de la production du bâti, avant tout pour mettre en place un principe de redistribution généralisé par la suite à d'autres domaines d'intervention.

On voit que le modèle urbain étatique tel qu'il a été plaqué dans les villes et les villages du Fezzan portait en germe de nombreux maux. D'évidence, les agglomérations sont sous-dimensionnées par rapport à la rapidité de la croissance démographique. Elles semblent avoir été pensées pour une société qui aurait maintenu son mode de vie antérieur dans un nouvel espace de vie, négligeant ainsi la fabrique sociale que représente l'épreuve de la ville. On ne peut alors être surpris de la crise du modèle 
urbain étatique, d'autant que celle-ci était déjà annoncée par les chercheurs durant la période d'extension du modèle.

En habitant ces villes pensées ailleurs, les populations du Sahara libyen ont en parallèle provoqué leur déclin... en même temps que l'émergence de nouvelles modalités de l'habiter. 


\section{CHAPITRE 8 - SEBHA, FACETTES DU COSMOPOLITISME SAHARIEN}

Comment un village parmi d'autres devient-il en quelques décennies l'une des plus importantes agglomérations de Libye mais aussi de tout le Sahara ? La croissance de Sebha est forte dès les années 1940 et ne ralentit pas par la suite. Entre 1931 et 1995, avec un croît démographique annuel de près de $7 \%$, la ville connaît l'une des progressions les plus importantes du Fezzan durant le $\mathrm{XX}^{\text {ème }}$ siècle. Cette croissance est dans un premier temps due à un exode rural massif alors que Sebha, centre colonial italien puis français, devient ville d'Etat après l'Indépendance pour être aujourd'hui la ville qui capte dans le Sahara les plus importants flux de migrants nationaux et internationaux. La succession de ces dynamiques de croissance fait de Sebha une ville plurielle, métropole régionale, nationale et saharienne. Une identité cosmopolite émerge dans un cadre urbain pensé ailleurs mais progressivement réapproprié par les populations qui l'habitent, le pratiquent et le signifient.

\section{LA PLANIFICATION URBAINE À L'ÉPREUVE DES MIGRATIONS}

L'évolution du tissu urbain de Sebha reflète les allers-retours entre la croissance soutenue que la ville connaît et la volonté de régulation de la part des autorités. Durant les années 1970, l'étude du cabinet Doxiades sur la ville de Sebha conduit à la mise en place d'un schéma directeur complété par l'analyse démographique italienne effectuée par Italconsult.

Sebha n'était qu'un modeste ensemble oasien aux lendemains de la Seconde Guerre Mondiale (fig.13). Ceci explique sans doute en grande partie les similarités entre son organisation et celle des plus petites agglomérations du Fezzan. Trois villages étaient disséminés dans la palmeraie de Sebha, Al Hajara, Al Jedid et Qourda, encadrés par un centre colonial administratif et militaire installé à proximité, sur une butte. Rapidement, des phases de migrations se succèdent, nourries par l'exode rural puis l'afflux de réfugiés et la fixation des nomades, contribuant à la croissance rapide de la population et à l'extension spatiale du bâti. La première phase d'installation, de villageois originaires du sud de la Libye, est résorbée par une intervention étatique vigoureuse. Comme ailleurs au Sahara, on assiste à l'émergence d'une « ville-champignon », agglomérat de grands ensembles, d'habitat précaire, de bâtiments publics et de casernes. 
En 1966, alors que Sebha compte moins de quinze mille habitants, un axe central structure le centre-ville et traverse l'agglomération de part en part, selon une direction sud-est/nord-ouest. Les principaux centres commerciaux et les bâtiments administratifs se situent le long de cet axe et les noyaux d'habitat, quoique étendus, sont groupés en cellules séparées les unes des autres. Un regroupement selon l'origine géographique (habitants du Shati à Al Mahdia, de Mourzouq à Jedid), s'effectue dans les logements collectifs construits par l'Etat, dont deux seulement se situent dans l'agglomération (Jedid et Qourda); le troisième, Hajara, est sans doute abandonné comme le laisse supposer la disparition de cette entité administrative entre les recensements de 1954 et 1964.

Figure 13 : Sebha, extension et migrations (1966-2000)

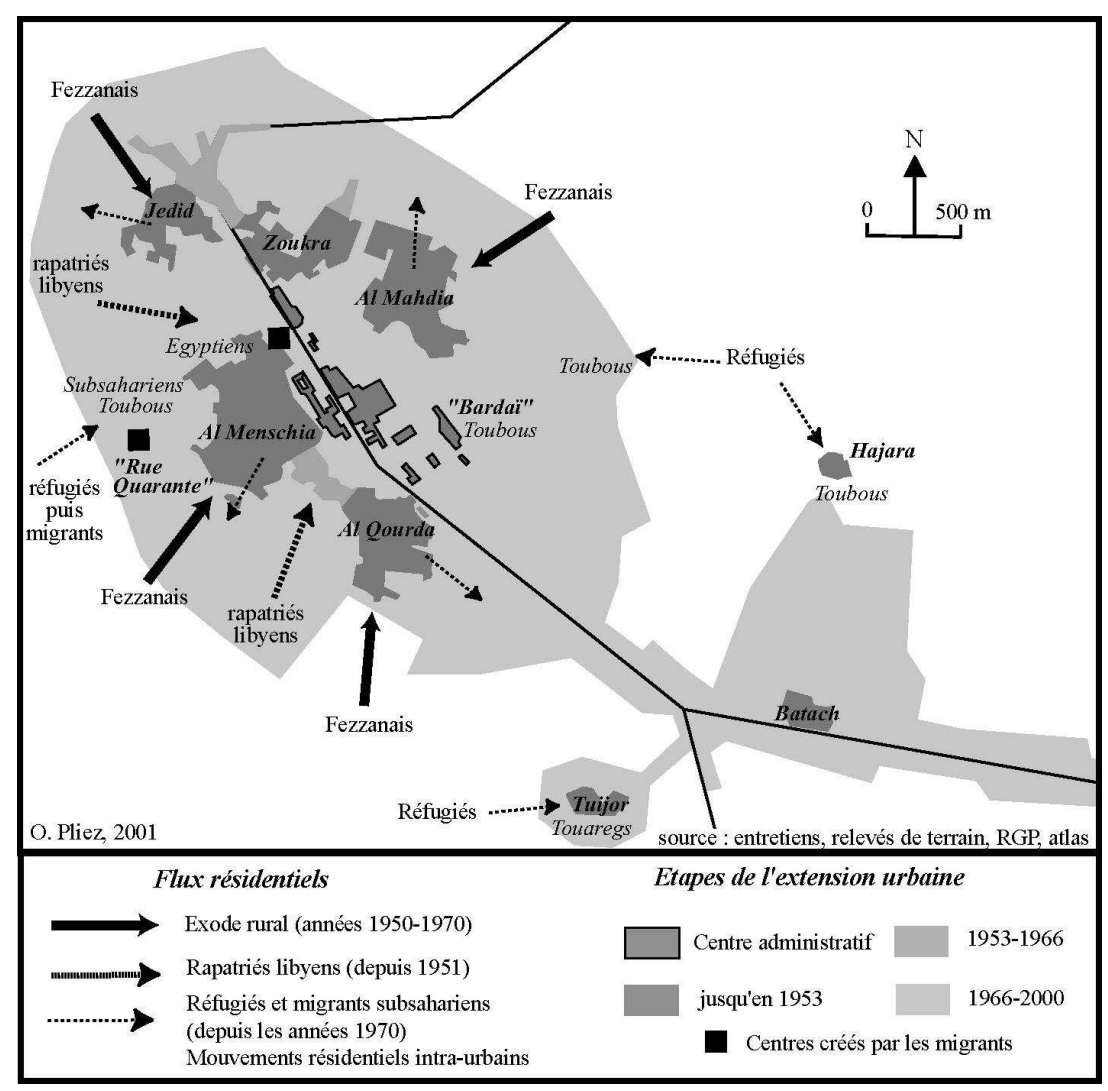

En 1981, l'étude menée par le cabinet finlandais Finnmap aboutit à un nouveau schéma à l'horizon 1988, dont l'ambition est clairement de 
réorganiser le tissu urbain en reliant les différents sites. Pour les auteurs de l'étude, quatre éléments doivent être pris en compte : mettre en adéquation la structure sociale communautaire et la ville, doter les nouvelles extensions urbaines de services de base, promouvoir la fonctionnalité de la ville et protéger les espaces agricoles. Cette déclaration d'intention, somme toute assez vague et générale, aboutit à l'élaboration de cinq scénarios d'occupation du sol. Finnmap préconise aux responsables libyens d'opter pour le modèle le moins coûteux et le plus simple à mettre en œuvre, celui qui ne remet pas en cause la structure existante puisqu'il vise à orienter l'étalement spatial en auréoles concentriques à partir de l'axe central.

L'extension de l'infrastructure routière devient l'élément structurant de Sebha. Autour de l'axe central, deux boulevards périphériques cerclent la ville, coupés par des axes perpendiculaires qui partent du centre vers la périphérie et correspondent à des «coulées vertes » où l'implantation des espaces verts a pour fonction d'aérer la ville mais aussi de matérialiser la séparation entre quartiers. L'appareil commercial se répartit en deux ensembles : au centre de Sebha, une vaste zone commerciale est prévue entre les deux axes parallèles, complétée par des bâtiments administratifs alors que dans les zones d'habitat, un petit centre commercial associé à un bâtiment de services administratifs et un jardin public est censé limiter les déplacements des habitants dans la ville.

Cette organisation ne remet pas en cause la phase précédente d'urbanisation spontanée; au contraire, elle la formalise et la met en cohérence avec l'extension du tissu urbain. Les regroupements communautaires, fondés sur l'origine, sont donc le mode d'habiter dominant de Sebha. La séparation des sites, et la centralité qui l'accompagne, donnent une structure similaire à celle d'un village où la juxtaposition des quartiers simule celle de villages que cerclent la palmeraie, les jardins et les espaces industriels, tous périphériques. En somme, le modèle de l'agglomération prime sur celui de la ville car au regroupement communautaire voulu par les populations et les autorités ne correspondent pas d'alternatives nettes qui viseraient à ce que se multiplient les interactions entre communautés d'origines géographiques ou tribales différentes, par exemple à travers la mise en place de lieux publics. Les autorités publiques se contentent de gérer la croissance en taille et en surface du tissu urbain de Sebha, laissant le soin aux Fezzanais et aux rapatriés libyens qui s'y installent de choisir les modalités de cette installation. 
A partir des années 1970 et surtout 1980, le basculement de flux migratoires d'abord d'origine régionale et progressivement internationale met à mal les principes proposés dans les schémas directeurs. Des milliers de Tchadiens et de Nigériens viennent s'installer en Libye, surtout à Sebha, à la suite des insurrections touareg au Niger et au Mali et de la guerre entre la Libye et le Tchad. Cet afflux massif de population contribue à une extension rapide de l'habitat informel périphérique. Et alors que la «première » ville de Sebha, fondée sur l'origine fezzanaise des populations commençait à se constituer, l'arrivée de réfugiés entraîne la naissance d'une «seconde» ville, dont le poids se renforce durant les années 1990 avec l'intensification des mobilités et des échanges transsahariens entre le Sahel et la Tripolitaine.

Les administrateurs locaux ne cachent pas leur trouble du décalage qu'ils ressentent entre les outils dont ils sont munis et l'évolution de la ville qu'ils observent à la fois comme acteurs et comme habitants. Les schémas directeurs et les dossiers d'études ne donnaient en effet aucune information sur les études sectorielles et de faisabilité, c'est-à-dire sur les outils permettant de juguler l'extension du tissu urbain. Pour autant, la structure duale n'est pas figée comme en attestent la diversification de l'habitat, les stratégies résidentielles et les pratiques quotidiennes de la ville par les citadins. Mais cette évolution échappe très largement à toute velléité d'intervention publique.

\section{LE QUARTIER, UNE UNITÉ FAMILIALE}

Le quartier a un statut intermédiaire entre la maison et la ville car il ne se réduit pas à une addition de maisons mais l'addition de quartiers ne fait pas non plus la ville. Dans le Sahara, c'est au niveau du quartier plus que de la maison, que la question de l'altérité se pose avec force en raison de l'étroite association entre les communautés et la structure urbaine. Le lien communautaire conduit à élargir le périmètre de l'aire où l'anonymat n'existe pas.

La segmentation urbaine est une modalité banale de la structuration sociale des agglomérations sahariennes, particulièrement lors du processus de fixation des nomades. L'installation de ces derniers s'effectue en fonction du côté par lequel le groupe nomade aborde la ville, c'est-à-dire dans l'axe des parcours pastoraux. Il en résulte une forte adéquation entre l'organisation de l'espace nomade et la structure des quartiers. Cette division s'atténue avec le temps car les déplacements et les mariages contribuent progressivement à une mixité spatiale et sociale. 
Afin de repérer les quartiers, on peut d'abord s'attacher à leur aspect visuel, à leur manière de contraster dans le paysage urbain mais ce repérage ne permet évidemment pas de distinguer entre eux les quartiers aux formes d'habitat identiques mais aux caractères sociaux différents. Le nom est un indice intéressant à repérer dans les nouveaux quartiers d'habitat ou ceux où de nouvelles populations se sont installées. Souvent, le village de fixation des nomades porte le nom du site sur lequel il s'étend, comme les quartiers Touaregs de Tilaqi à Oubari et d'al Karama à Sebha. En périphérie du projet agricole d'Oubari, le village Toubou prend plus simplement le nom de Projet. Le nom se référant à l'origine du groupe installé se retrouve souvent, en particulier à Sebha: un collectif très dégradé, où furent installés des réfugiés du Tchad, est appelé Bardaï, du nom de la principale bourgade du Tibesti ; de même, la rue principale du quartier africain est appelée Rue Quarante, en référence à la rue centrale de N'Djamena.

Tous les quartiers péri-centraux de Sebha présentent la même configuration : on passe du goudron au sable, en rupture avec les axes intra-urbains et le bâti est très hétérogène à l'intérieur même de l'îlot contrairement aux quartiers et aux villages où l'homogénéité de l'habitat est très prégnante. Ici quelques résidences privées de haut standing jouxtent des logements publics, très largement transformés, et des surfaces où l'autoconstruction s'étend, dans les interstices laissés libres entre les deux autres types de logements. Aux périphéries de ces quartiers, c'est-à-dire le long des axes de circulation de la ville, les commerces occupent le rez-de-chaussée des demeures.

Cette hétérogénéité qui se répète si fréquemment est l'indice d'une structuration familiale du quartier. Le passage du «goudron» au sable matérialise la frontière, plus que la limite, entre espaces publics et privés, du moins semi-privés. Cette rupture, le promeneur la ressent lorsqu'il pénètre dans l'îlot; à peine s'y est-il engagé que l'un des commerçants vient à sa rencontre, le questionne sur sa présence. Cette impression de rupture est confortée par le nombre d'enfants qui jouent seuls dans les ruelles.

Les mariages sont l'occasion de marquer l'unité du quartier car ils se déroulent à l'extérieur plutôt que dans les maisons. Des bâches sont tendues dans les rues, entre les murs afin de dresser une tente pour les hommes et une pour les femmes (photo.10) qui serviront après la fête de lieu de repos pour les invités extérieurs à la ville. Or cette sortie du domicile ne pourrait s'effectuer directement dans la rue. C'est donc une 
véritable recréation d'espaces intermédiaires à l'intérieur même du tissu urbain qui s'opère, en somme une privatisation de l'espace proche.

Cette structuration familiale n'empêche pas l'émergence d'une hiérarchisation sociale marquée, sans pour autant contribuer à l'éclatement de la cellule familiale. Au contraire, les résidences les plus imposantes appartiennent aux membres les plus aisés du groupe ; elles sont donc aussi celles de la plus importante sociabilité, les lieux où l'on se retrouve pour les repas ou les loisirs.

Photographie 10 : La tente de mariage, une appropriation temporaire de la rue

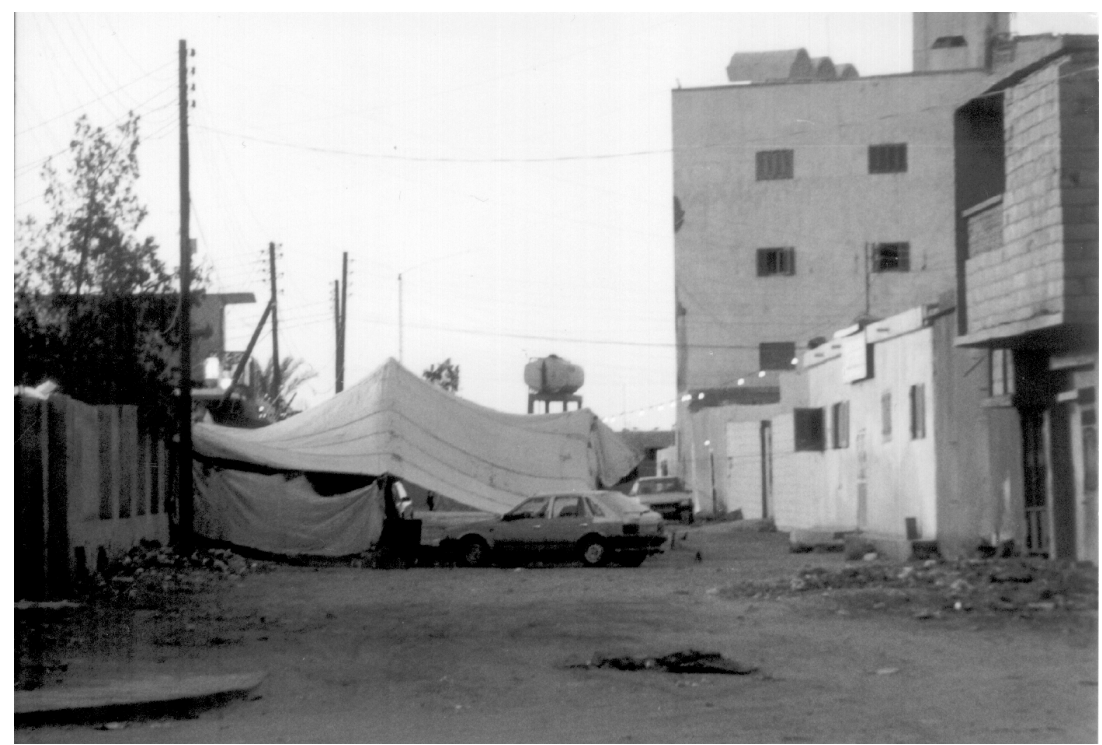

La morphologie de base des quartiers se composait de résidences d'Etat et de terrains vagues mais ces derniers sont désormais en voie de comblement. Le développement du résidentiel privé y est pour beaucoup, de même que la location de baraques autoconstruites aux immigrés qui permettent de les loger sur place et d'assurer des compléments de revenus. Enfin, les boutiques se multiplient pour répondre au même besoin et si elles ne sont pas tenues par des membres de la famille, elles le sont par des immigrés logés dans l'îlot. Les fonctions du quartier se diversifient donc rapidement, tout en conservant la même assise familiale. 
Cette organisation contribue aussi à une gestion familiale du foncier et conduit à pointer les délicates relations entre l'Etat et les autorités coutumières. En effet, dès 1952, un an après l'Indépendance de la Libye, les autorités procèdent au recensement des propriétés, un acte sans conséquences car la population continue à appliquer le droit coutumier pour l'acquisition ou la transmission des terres, c'est-à-dire sur la reconnaissance d'une propriété après mise en valeur ou installation. Après 1969 et la prise du pouvoir par Mouammar Kadhafi, différentes lois sont promulguées afin de supprimer la propriété tribale des terres. En 1985, l'Etat annule tous les enregistrements de titres de propriété et institue un domaine public sur les terres non utilisées. Dès lors, tous les propriétaires doivent faire enregistrer leur bien pour bénéficier d'une reconnaissance officielle de leur statut or les enregistrements ne commencent que depuis deux ou trois ans à Sebha alors que les autorités de l'Etat les accompagnent de gratifications substantielles telles que l'attribution d'un lot de $500 \mathrm{~m}^{2}$ pour une construction et des incitations financières sous forme de prêt sans intérêts ou de subventions. Ce retard s'explique par le fait que les tribus les plus puissantes, qui peuvent se dispenser des aides, continuent à procéder selon le droit coutumier. Ce moyen de revendiquer leur droit premier sur la terre entraîne un processus de privatisation des terres du domaine public qui changent de facto de statut juridique. En revanche, ce face-à-face limite les marges de manœuvre des autorités publiques locales, lesquelles ne peuvent dans ces conditions que difficilement penser et mettre en œuvre une politique urbaine cohérente à l'échelle de la ville de Sebha.

Finalement, l'hétérogénéité du bâti cache une dynamique fondée sur la consolidation de la structure familiale parallèle au processus d'urbanisation. La situation peut sembler paradoxale puisque l'on trouve de manière accrue à Sebha, grande ville de création récente, image apparente de la «modernité saharienne », ce qui semblerait n'être qu'une manifestation d'un conservatisme cantonné aux villages oasiens. Or c'est tout le contraire que l'on observe, de toute évidence parce que la taille de l'agglomération s'accompagne d'adaptations afin de protéger la structure familiale face aux « risques » de la grande ville, l'anonymat, l'éclatement du groupe et l'atténuation du contrôle social.

\section{DES CENTRES CRÉÉS PAR LES USAGERS DE LA VILLE}

Les planificateurs n'ont pas négligé les centres-villes puisqu'ils étaient dans leur vision de la ville construite par les pouvoirs publics, le lieu de concentration des fonctions de pouvoir, d'administration et de commerce dans un contexte de nationalisation des activités. La ville pensée ailleurs a 
donc été dotée d'un centre fonctionnel, établi à partir du modèle -même s'il peut laisser l'observateur extérieur dubitatif- du CBD, le Central Business District des villes nord-américaines. On devine les intentions sous-jacentes à cette démarche : créer la ville, c'est nécessairement la doter de centres qui n'existaient pas jusque-là. En revanche, la référence au CBD surprend tant son décalage est flagrant avec ce qu'est la ville de Sebha, ville somme toute moyenne avec environ 120000 habitants, ville de l'altérité par rapport aux lieux où les modèles ont été pensés. Le porte-à-faux entre le prétendu « universel urbain » et la réalité a produit ici l'inverse du résultat escompté, le centre étant en fin de compte un «non-lieu » vers lequel les citadins ne se déplacent que lorsqu'une raison précise les y force.

Si ce «non-lieu» n'a pas fait l'objet d'un investissement social, symbolique ou pratique de la part des habitants, en revanche plusieurs centres jouent un rôle actif au plan fonctionnel et comme lieux d'interaction sociale. Dans cette optique, les nouvelles centralités constituent un des indices majeurs de l'urbanité émergente et traduisent quelques-uns des traits saillants de la société urbaine de Sebha, composite et cosmopolite.

A Sebha (fig.14), le centre ville institutionnel se matérialise par la présence d'immeubles modernes, d'hôtels, de la poste, des principaux bureaux administratifs et bancaires. Le réseau commercial, en revanche, est réduit, dominé par les librairies et la bureautique car les commerces se répartissent sur différents axes de la ville, selon une spécialisation marquée : grossistes en produits alimentaires à proximité du principal souk aux légumes; bijoutiers sur une avenue des quartiers plus aisés; équipements ménagers sur une portion de l'axe central et vêtements dans trois sites distants les uns des autres. Le fait qu'une ville de taille réduite comme Sebha se caractérise par une telle polycentralité relève de deux raisons principales, l'une renvoie à l'évolution des positions du régime vis-à-vis du commerce de détail, un temps prohibé et l'autre renvoie à la place de Sebha dans le commerce avec l'Afrique.

La planification de la ville de Sebha est en effet mise en œuvre durant les années 1980, c'est-à-dire dans un contexte de nationalisation des activités économiques. Le commerce de détail n'existe plus que sous la forme des souks d'Etat, sortes de grandes surfaces où l'ensemble des produits vendus aux particuliers est regroupé. Le développement du commerce privé de détail est postérieur à la période de la création du centre-ville, qui en conséquence n'avait pas été conçu pour intégrer ces activités. La coïncidence entre la localisation des quartiers commerçants et 
des quartiers résidentiels s'explique par le fait que la majorité des boutiques sont attenantes à la demeure où vit le propriétaire.

Figure 14 : Sebha en 2000, la morphologie urbaine

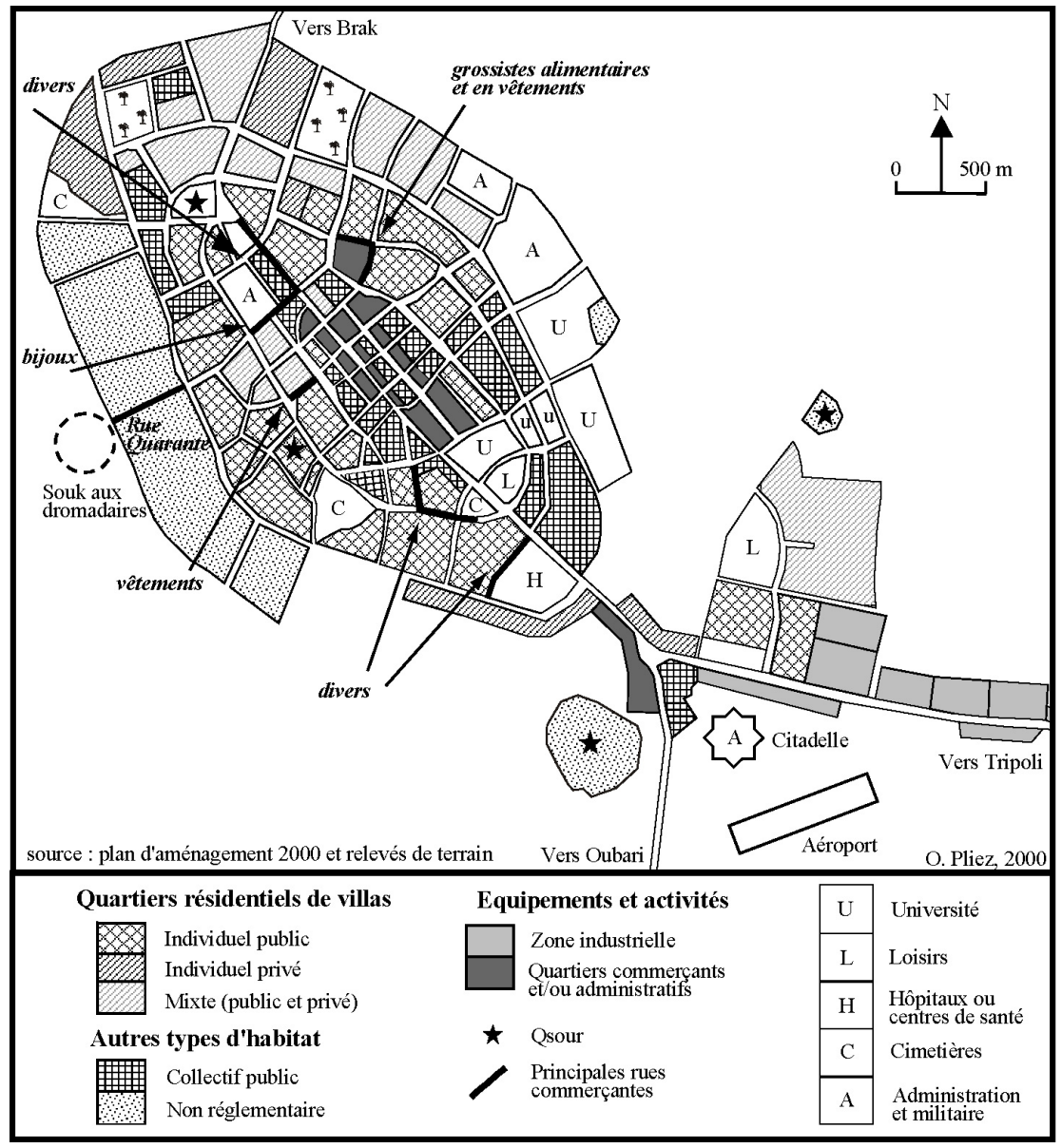

En outre, Sebha est une grande place commerçante entre la Libye et les Etats d'Afrique subsaharienne, en même temps qu'une destination pour les migrants du Monde arabe. Ce cosmopolitisme rejaillit sur la structure polycentrique de la ville puisque le centre commerçant populaire est égyptien, le centre des échanges est africain et les autres sont libyens. Dans les deux premiers cas, produire de la centralité, c'est créer un lieu où l'on peut échanger des produits circulant dans les réseaux commerciaux tissés 
par les migrants et les commerçants transnationaux de même nationalité ou de même origine régionale (Sahel, Afrique du nord) mais c'est aussi créer un lieu de sociabilité fondé sur cette origine géographique commune.

Le cas des Egyptiens est le plus éclairant. Très présents dans le secteur agricole, ils investissent aussi bien les activités de production que de commercialisation des fruits et légumes. C'est donc à la charnière entre la rue centrale de Sebha et le principal marché de la ville qu'ils sont les plus présents. Ce secteur est d'abord un lieu de travail pour ceux qui acheminent les produits agricoles depuis les fermes périurbaines ou les vendent sur les étals mais aussi pour les journaliers, assis sur le bord du trottoir, le long des avenues, tenant au bout d'une tige de fer l'outil qui renseigne sur leur métier les employeurs potentiels qui circulent en voiture. Rapidement, les autres dimensions de ce lieu apparaissent avec d'abord la présence de quelques cafés animés et ouverts sur l'extérieur, où les clients fument le narghilé en regardant les programmes égyptiens sur les télévisions mais aussi des restaurants qui proposent des plats égyptiens. La situation, autant que l'ambiance qui se dégage de ce lieu, en fait aujourd'hui un point de rencontre des Egyptiens mais aussi des Libyens dans la ville. Ils y viennent voir des films en terrasse, parler ou jouer avec leurs amis.

La Rue Quarante est la principale rue des quartiers périphériques de Sebha qui tient son nom d'un des axes de N'Djamena, au Tchad. La Rue Quarante a plusieurs fonctions, commerçante avec la présence de petits commerces et de deux bazars; de transit avec le Sahel puisque c'est là qu'arrivent les camions chargés de produits et de travailleurs à destination du Tchad et du Niger depuis et vers Agadès ; d'approvisionnement car le bétail destiné au marché libyen y est acheminé depuis le Niger et le Tchad. Au-delà de cet aspect fonctionnel, la rue Quarante est aussi le pendant à l'austérité de la ville. Animée le soir, elle est le lieu de passage, à pied ou en voiture, de ceux qui recherchent l'animation d'un quartier dont l'ambiance diffère totalement. Les commerces, la population, l'organisation et même le nom renvoient à l'Afrique subsaharienne. Sas pour ces populations, elle est pour les Libyens un espace de la marginalité, rejetée dans le discours, attractive au quotidien. Quartiers des trafics et de la prostitution, elle participe des principaux lieux de sociabilité de Sebha. La présence étrangère contribue à l'émergence de lieux publics qui font peu à peu partie intégrante de la ville.

La centralité des quartiers commerçants libyens diffère de celle des quartiers à population migrante. Elle est guidée par la localisation des espaces résidentiels puisque généralement les boutiques sont ouvertes sur 
le rez-de-chaussée du domicile privé ou regroupées par corporations. Sa pratique spatiale est d'abord familiale ou féminine, essentiellement en début de soirée car c'est rarement avant la tombée de la nuit et après l'heure du dîner que les Libyens ouvrent ces commerces. La mixité de l'espace prend réellement sens dans les quartiers aisés à population libyenne dominante et à proximité du domicile familial, c'est-à-dire à la condition que le contrôle social soit assuré.

La polycentralité traduit donc les différences sociales entre les citadins de Sebha. Chacun de ces centres a une dimension fonctionnelle, mesurable et cartographiable à partir de la répartition des commerces, mais elle est aussi « fabrique » de lieux de sociabilité, indispensables à ceux qui vivent ici autant qu'à ceux qui y passent.

Le quartier semble être cette unité élémentaire de la communauté, une structure intermédiaire entre l'individu et la ville. A Sebha, l'ouverture de la maison sur l'intérieur que forme le quartier coïncide avec une fermeture sur l'extérieur, la rue, confirmant ainsi l'idée d'une adéquation entre la juxtaposition des quartiers de la ville et celle des communautés. A ce niveau spatial, on peinerait à chercher des indices probants d'urbanité.

Si Sebha a été créée par l'Etat, les centres le sont par les habitants parce qu'alors que la ville est d'abord la traduction spatiale d'un plan d'aménagement, les centres, au contraire, se caractérisent par leur décalage avec la structure urbaine initiale et leur forte relation avec l'organisation sociale de la ville. L'émergence de nouvelles centralités contribue à souder le tissu urbain puisqu'elle participe d'une dynamique d'ouverture qui contraste avec celle, plus nettement fermée, plus protégée aussi, des quartiers. Cet effet de contrepoids, ou d'équilibre, incite à pousser plus avant le parallèle entre les deux mouvements. Désormais, les restructurations urbaines d'initiative publique et privée sont conjointes. La croissance de l'habitat et des locaux privés se traduit à Sebha par une privatisation du cadre urbain au profit des groupes qui l'habite. L'émergence d'espaces publics, lieux du négoce et des sociabilités, apparaît comme un contrepoids qui reflète la diversité croissante du bâti et des populations. Ces deux dynamiques, d'apparence contradictoire, renvoient en définitive à une seule réalité de la ville. 
CHAPITRE 9 - L'URBANITÉ SANS VILLE DU WADI AL HAYA

Hors du Sahara et de son aire d'influence directe, peut-on encore évoquer le terme d'urbanité ou bien la présence des rubans de palmeraies confine-t-il les vallées oasiennes dans la ruralité ? L'ajout du suffixe -ité cache mal la tentation du chercheur à opérer une séparation entre l'urbain et le rural, les villes et les campagnes. Il paraît nécessaire de rappeler quelques faits. En 1995, plus d'une personne sur deux dans le Sahara libyen vit dans une agglomération de 1500 à 10000 habitants. Le processus d'urbanisation a donc été dans le désert un processus de micro-urbanisation par création et/ou croissance démographique de centres petits et moyens.

L'exemple du Wadi Al Haya illustre bien les caractères de ce processus. Dans un premier temps, le mouvement d'urbanisation-modernisation mené du haut vers le bas, s'exprime à travers une politique d'aménagement fondée sur la rénovation rurale de la région et la promotion de centres administratifs. Mais progressivement, à travers leurs pratiques quotidiennes et routinières, les habitants du Wadi Al Haya s'approprient cet espace de vie façonné par des interventions extérieures ; ils le signifient en le transformant, construisent un discours qui reflète des représentations collectives, véritables clés de compréhension du quotidien dans les espaces oasiens.

Pour autant, la lecture selon laquelle un mouvement du bas vers le haut se substituerait à un mouvement inverse, jusqu'alors prédominant, du haut vers le bas, reste évidemment schématique. Dans un cadre fixé par d'autres, les Fezzanais redéfinissent leur rapport à l'espace sous l'expression d'une urbanité qui se déploie dans un réseau villageois, en somme, une urbanité sans villes.

\section{CONSTERNANTS PAYSAGES URBAINS !}

La rapidité -oserait-on dire la brutalité ?- du processus d'urbanisation a laissé plus d'un observateur perplexe. L'uniformisation des paysages, tout comme la rapidité des changements spatiaux et sociaux ont été à l'origine, en réaction, d'un discours nostalgique sur la ville. D'As Smara, dans le Sahara occidental, Le Clézio (1997) donne une description très évocatrice. «Au-dessus de la vallée, la citadelle est en ruine. Elle est flanquée de casernes, de garnisons; une grande antenne de radio jaillit des anciens remparts. La vaste agglomération de tentes en peau de chameau... a laissé 
la place à des constructions précaires en parpaing, coiffées de dômes blancs. Il y a un souk où les commerçants venus des quatre coins du Maroc vendent des tissus, des comestibles. Le palais de la Province domine la ville de ses hauts murs de pierre noire. Il y a des avenues rectilignes, des trottoirs, quelques maigres jardins. La cité mystique s'est changée en garnison militaire et en centre commerçant...». Le regard distancié que posent les écrivains sur un village du Sud marocain, où ils arrivent après un long et onirique trajet automobile, nous permet de souligner la difficulté à laquelle est confronté le géographe face à son objet d'étude. Ce bourg saharien, à travers les yeux d'un romancier plus soucieux de capter le sensible que le réel, n'est qu'un artefact qui semble n'avoir été fait ni par ni pour ceux qui y vivent. De même, avec une pointe d'ironie, Mustapha Boudiaf (1994) constate qu'El Oued, principale ville du Souf, dans le Sahara algérien «a adopté le béton... Ici, comme ailleurs, c'est l'architecture nationale qui domine... à telle enseigne qu'on a l'impression de ne pas bouger quand on voyage ».

Mais une fois le constat posé, quelle lecture sociétale faire de ces «non-lieux » ? La diffusion d'un modèle standard, imposé par le haut, a souvent été interprétée comme un facteur de remise en cause des traditions et valeurs villageoises. Mohamed Naciri exprime bien cette tendance à travers l'étude d'un village du Sud marocain effectuée au début des années 1980. Son analyse sur les causes du déclin rapide du mode de vie «ksourien» mettait l'accent sur l'importance de la route, $\mathrm{y}$ «transitent hommes et produits... l'argent mais aussi les modèles, les valeurs, les aspirations nouvelles ». La nouvelle agglomération, dénommée Nimirou (numéro) «par référence au chiffre de la borne kilométrique » symbolise cette tendance de l'urbanisation à fondre les villages dans «l'anonymat, l'uniformisation et la monotonie ». Ici, comme cela a souvent été le cas dans les espaces ruraux marginaux du Maghreb des années 1960 aux années 1980, les émigrés ont impulsé un mouvement d'urbanisation que l'Etat a accompagné en construisant des axes routiers. En effet, grâce à leurs revenus, ils ont investi dans la construction d'une villa, hors du ksar, le long de l'axe, suivis par les autres familles. Ce processus banal fut souvent perçu au plan local comme par les observateurs extérieurs comme le premier signe du délitement social. Toutefois, la lecture du processus proposée ici est symptomatique du discours dominant dans un contexte de bouleversement total des «campagnes sahariennes». Le mouvement spatial a partout conduit à tirer des conclusions sur le changement social, individualisation des comportements, anonymat, perte des référents identitaires «traditionnels», au détriment de l'organisation communautaire ou de l'équilibre de la cellule que représentait le ksar. 
Quel regard porter alors que l'achèvement de la transition urbaine se dessine dans l'ensemble du Sahara maghrébin ? La route a un effet structurant, spatial autant qu'imaginaire, mais symbolise-t-il pour autant la dissolution des identités locales dans un ensemble uniforme?

\section{VISIONS ET DISCOURS DES HABITANTS DU WADI}

On parle souvent de réappropriation matérielle de l'espace mais l'idée même de réappropriation est multiforme et dépasse la simple transformation du cadre bâti ; elle est aussi un processus selon lequel chacun tend à redéfinir ses repères dans l'espace vécu. Les discours révèlent quelques biais selon lesquels s'effectue cette réappropriation discrète et conduisent progressivement à chercher une définition de l'urbain par ceux qui le vivent ${ }^{22}$.

\footnotetext{
${ }^{22}$ Dans cette optique, afin de disposer d'un corpus de données cohérent, j'ai recueilli des informations par entretiens et questionnaires. Les entretiens, rarement formalisés, sont le résultat de rencontres avec des étudiants, des jeunes engagés dans la vie active, des responsables locaux, parfois des travailleurs immigrés, originaires d'Afrique de l'ouest, du Sahel et du Monde arabe. J'ai obtenu auprès d'eux des informations très diverses sur leur quotidien, leurs pratiques, leur vision de la région, du village, de la route, des touristes. Ces éléments m'ont permis de mieux cerner les spécificités du Sud libyen par rapport à ce qui se dégageait de mes lectures sur le reste du Sahara maghrébin. Néanmoins, j'ai ressenti le besoin de savoir si ces informations étaient représentatives de ceux qui, polyglottes, souvent diplômés, s'étaient prêtés aux entretiens ou bien si elles reflétaient une tendance générale hors de ce cercle étroit. En effet, les entretiens, à la fois informels et formulés à l'écart du groupe, étaient pour certains un exercice d'expression libre ou bien de propagande appuyée ; le risque d'une certaine artificialisation de ce corpus d'informations n'était pas négligeable.

J'ai donc soumis 26 questions à 41 personnes qui résident pour l'essentiel dans le Wadi Al Haya occidental, de Gabraoun Jedid à Oubari. Les différents niveaux de la hiérarchie des agglomérations sont représentés, quoique très inégalement, dans un secteur qui a largement bénéficié des sollicitudes de l'Etat et connaît aujourd'hui des taux de croissance encore élevés.

Les résultats obtenus sont très mitigés. En effet, il n'a pas été possible d'interroger de femmes et les étrangers ont généralement décliné. Leur situation délicate les pousse plus facilement à s'exprimer à l'oral. Autre élément limitant, «la sincérité » des réponses qui s'assimilent parfois à une vulgate officielle. Plus de la moitié des enquêtés sont engagés dans la vie active, les autres se répartissant de manière équitable entre retraités et lycéens ou étudiants. Dans la mesure où nous n'avons pas de questionnaire d'immigrés, la part des fonctionnaires dans le total des actifs est élevé, 19 sur 25, dont trois reconnaissent pratiquer une activité dite complémentaire, souvent principale dans les faits. Les actifs du secteur privé sont donc peu représentés.

L'exploitation systématique s'est donc avérée impossible à mettre en œuvre et a rendu caduque les regroupements de question trop formels, finalement remplacés par d'autres,
} 
Paradoxalement, si le nom Fezzan est bien présent dans le registre du tourisme, pour qualifier des circuits ou des enseignes d'agences de voyage... il n'est pas sûr qu'il connaisse le même succès dans la région qu'il est censé qualifier.

La question des limites du Fezzan ne pouvait que surprendre les enquêtés et les conduire en fait à formuler les contours de l'espace régional dans lequel eux se reconnaissent (fig.15). Néanmoins, elle permettait de distinguer deux manières de voir l'ensemble régional, l'une élargie, l'autre restrictive. La vision élargie renvoie à un vaste territoire s'étendant de Ghadamès au Nord à la frontière avec le Niger au Sud alors que la vision restrictive se limite au noyau central, de Sebha à Oubari et Mouzouq. Le Wadi Ash Shati est dans ce cas rarement évoqué, moins en tout cas que Ghat, pourtant beaucoup plus distante du Wadi Al Haya.

Ces éléments nous permettent de conforter la prégnance de deux conceptions territoriales fondamentales. La vision élargie correspond peu ou prou à celle du Fezzan» historique », c'est-à-dire un carrefour saharien. Est-il étonnant que ceux qui le représentent ainsi soient surtout les personnes âgées et les plus diplômés ? Ils restituent alors un cadre appris plus que vécu. Par contre, les jeunes réduisent le territoire à celui de leur pratique quotidienne et occasionnelle. «Leur » Fezzan se réduit alors à une portion du Sahara libyen méridional.

\section{Figure 15 : Quatre visions du Fezzan}

En haut, deux représentations contrastées, révélatrices d'un clivage générationnel : en haut à gauche, un jeune homme réduit le Fezzan à l'espace de ses pratiques, c'est-à-dire le long de l'axe routier et des agglomérations qui le jalonne. Le Shati, au nord et l'Est de la Dépression de Mourzouq sont exclus. Notons, en outre, qu'elles correspondent respectivement au "Fezzan arabe", lié à la Tripolitaine au nord et au "Fezzan toubou", lié au Tchad et au Niger vers le sud-est. A droite, on retrouve une vision plus classique, celle du "carrefour saharien".

En bas, deux croquis : à gauche, le Fezzan dans ses limites administratives ; à droite, le Fezzan reconstitué dans une représentation de l'espace nomade, les agglomérations se substituent aux autres repères comme points d'ancrage spatiaux. Ici, seules sont représentées celles comptant une communauté touarègue notable ou majoritaire, finalement assez proche de la représentation "routes et villes".

thématiques. Des réponses ont rapidement révélé le caractère artificiel de certaines séparations, celle par exemple entre la région et le village. Souvent, on n'obtient de réponse qu'à l'une ou l'autre des questions sur ces thèmes ou bien une redite. L'élément est intéressant en soi puisqu'il permet de supposer que le village n'est pas toujours un cadre pertinent de réflexion, du moins dans le contexte d'un réseau villageois dense ; mieux vaut y substituer la vallée. 


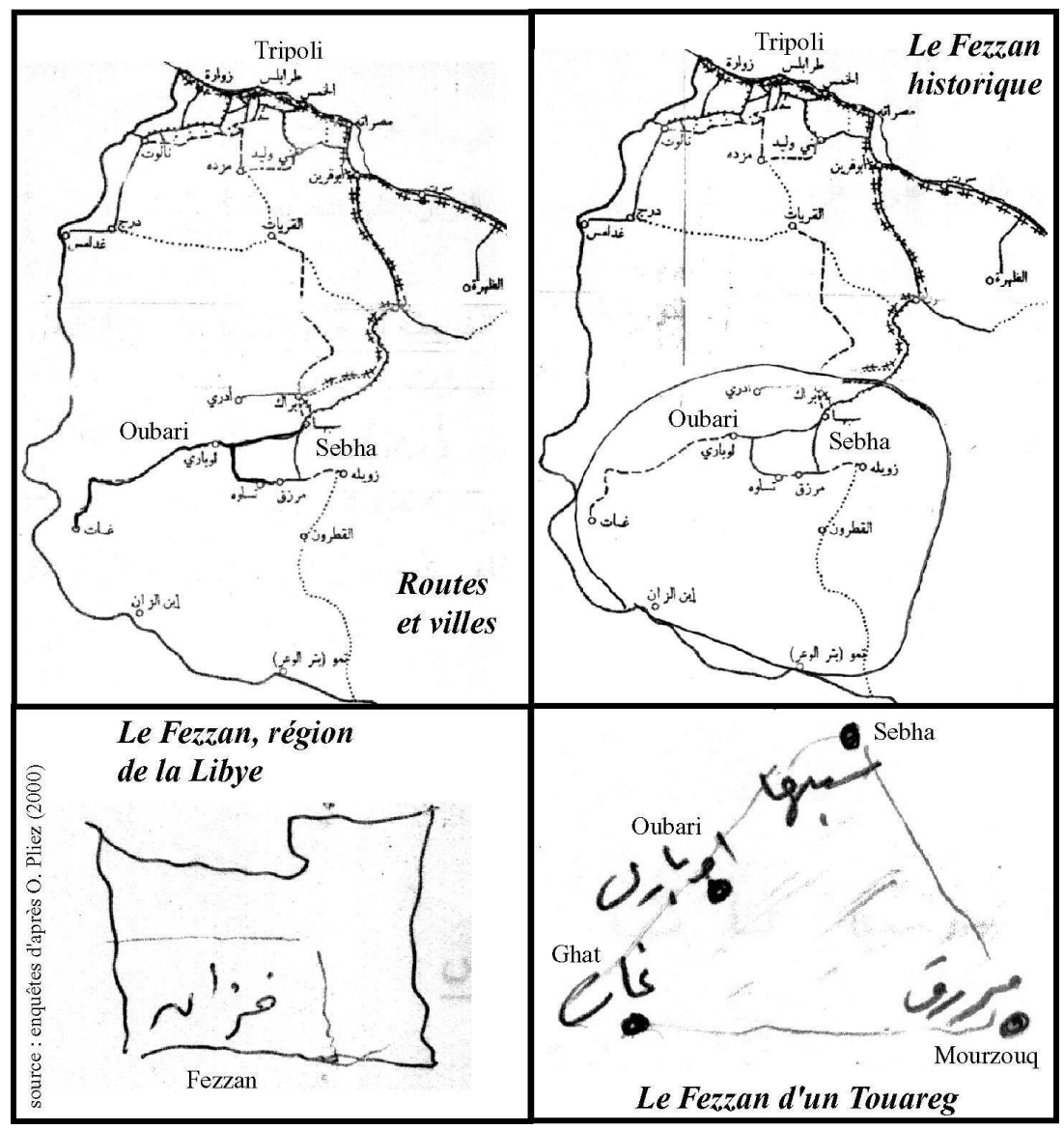

Ces deux conceptions du territoire s'accompagnent à nouveau d'une dualité dans les repères choisis pour le borner. La vision élargie associe les qualificatifs d'erg, de jardins, de vallée, en somme, une vision naturaliste qui correspond bien à une certaine idée de ce qu'est le Fezzan : une permanence, renvoyant au passé mais sans réel lien avec la situation qui prévaut aujourd'hui. La vision restrictive, par contre, s'appuie tantôt sur les routes, tantôt sur les agglomérations principales, voire les deux à la fois, c'est-à-dire sur les repères mis en place par les différents plans d'aménagement et l'urbanisation rapide.

La vision du territoire d'un jeune Touareg est intéressante car elle s'inscrit à la fois dans l'une et l'autre des représentations. En effet, on devine une proximité entre cette représentation et le fait que « pour décrire 
son territoire, un nomade dessinera sur le sable les vallées et les puits qui s'y inscrivent... le puits représente un véritable « centre » de rencontre, un point nodal entre plusieurs fractions ou tribus nomades» $(\mathrm{H}$. Claudot-Hawad, 1996). Or la transcription graphique triangulaire représente ce territoire nomade, à ceci près que les agglomérations s'y substituent aux puits.

Ces deux représentations du territoire traduisent deux glissements majeurs : glissement d'échelle lorsque à un niveau d'identification supposé régional, le Fezzan, se substitue un espace vécu essentiellement local ; glissement des repères lorsque à ceux, "immuables », de la nature se substitue le cadre spatial produit par l'Etat par le biais d'une politique d'aménagement volontariste. Ainsi, la rétraction du territoire qui s'esquisse à travers les représentations témoigne de l'intensité de la territorialité à l'œuvre. Le clivage est générationnel mais révèle sans doute aussi la propension des sociétés sahariennes à digérer les interventions extérieures, principalement l'urbanisation et le désenclavement, en procédant progressivement à leur réappropriation.

Ce qui s'apparente, vu de loin, à un mouvement d'urbanisation des campagnes, déstructurant et uniformisant, prend-il alors dans le discours de ceux qui sont censés le subir la même tonalité ?

Invitées à énumérer les mots qui permettent de qualifier la ville et l'urbain, les personnes enquêtées ne distinguent pas le fait dans ou hors du Sahara. Pas de spécificité saharienne, donc, mais une définition presque unanime de ce que signifie l'urbain. En effet, qu'il s'agisse des habitants (cité 18 fois), des voitures et des embouteillages ou des commerces ( 10 fois chacun), des immeubles et de l'habitat (9 fois), presque systématiquement leur sont accolés les qualificatifs de « trop, «beaucoup », « les uns sur les autres ». Ils révèlent une définition de la hiérarchie urbaine fondée sur l'entassement et la quantité. En somme, plus la ville est grande, plus les habitants s'y entassent, plus les immeubles, les usines, les embouteillages sont nombreux. Cette perception négative ne concerne pas l'ensemble des agglomérations mais seulement les plus grandes, Sebha, centre régional comptant plus de 100000 habitants, comprise. La transformation des vallées sahariennes n'est assimilée à ce mouvement que par un biais particulier. En effet, les entretiens et questionnaires laissent transparaître une représentation assez largement partagée de l'espace: on y trouve désormais les attributs de la ville mais à faible dose. Ce qui apparaissait à l'observateur extérieur comme un trait majeur des paysages, la négation de la ville, se réduit alors à un inconvénient mineur. Ainsi, si l'uniformité de l'habitat est nettement perçue, elle se justifie comme le pendant inévitable 
du confort. L'habitation «populaire », ou de manière plus évocatrice «salubre» $(s a h \hat{\imath})$, terme selon lequel les personnes qualifient leur domicile, devient alors pour ceux qui en possède une l'un des critères majeurs de la qualité de vie.

Les représentations de la nature confortent cette conception de l'urbain. Les vocables relatifs à l'agriculture tels que ferme, agriculture ou oasis sont très rarement mentionnés, de même que ceux évoquant la vie villageoise traditionnelle puisqu'il n'est question qu'une seule fois d' » union sociale » pour qualifier les villages. Au contraire, le discours se construit autour d'une idée forte : ici, on bénéficie aussi du confort mais sans pâtir des inconvénients de la ville. Pourquoi ? Parce que le Sahara libyen cumule les éléments qui étayent l'argument, à leurs yeux, de «bonnes conditions de vie », le calme (9 fois cité), l'air pur (6), le repos (5). Ces éléments sont à mettre en relation avec ceux qui concernent la définition de l'urbain car ils s'opposent à ce qui caractérise le désagrément de la vie citadine. La fréquence avec laquelle ces termes reviennent correspond sans doute à une volonté d'opposer deux modes de vie.

Une vision inversée de l'urbain se dessine progressivement, en porte-à-faux avec la vision extérieure, parfois plaquée sur la réalité villageoise.

Il est admis, tacitement, que l'urbanité est d'autant plus forte et évidente que la taille des agglomérations et la densité urbaine d'un espace donné sont importantes. Mais si l'on poursuit ce raisonnement jusque dans des espaces dont la marginalité semble indiscutable, des problèmes d'interprétation se posent. Ainsi, il apparaît que partir de la strate des métropoles et descendre jusqu'au niveau des plus petites agglomérations conduit à constater un appauvrissement progressif de l'urbanité des lieux observés. On retrouve une représentation classique des hiérarchies urbaines, celle d'un continuum partant du haut, les mégalopoles, vers le bas, le «rural profond», dont l'urbanité serait en fin de compte niée. Paradoxalement, alors que le consensus se noue autour de l'absence de pertinence du clivage ville-campagne, de manière plus discrète, le Sahara pourrait se trouver relégué dans une ruralité aux contours flous.

A cette vision, les enquêtés en renvoient une autre. L'urbain n'est pas perçu ici du haut vers le bas, prisme bien négatif et peu valorisant pour ceux qui le vivent à «la base », mais du bas vers le haut. Ceci semble logique et peut n'apparaître que comme un banal renversement de perspectives mais il faut peut-être préciser les conséquences de cette perception. On pourrait les résumer en disant qu'à la base de la hiérarchie se trouvent les attributs élémentaires de l'urbain ; plus on monte, plus ils se 
pervertissent par accumulation d'inconvénients inhérents à la (grande)

ville, ceux cités par la plupart des personnes.

Il ne s'agit évidemment pas de présenter une approche comme plus pertinente que l'autre mais de tenter de cerner une autre manière de percevoir l'urbain. Paradoxalement, s'il y a urbanisation des campagnes, et on mesure maintenant les précautions qu'il faut prendre pour accompagner l'utilisation du terme, on compterait plus de détracteurs du mouvement parmi ceux qui l'observent et le commentent que parmi ceux qui le vivent. N'y a t-il pas là un décalage paradoxal, entre la vision d'un espace idéalisé par ceux qui en sont extérieurs et la revendication de sa matérialité par ceux qui le pratiquent quotidiennement? En fait, le discours sur l'urbain dans le Fezzan se calque sur ce que l'Etat a créé puisqu'en disant urbain, on parle du confort et de l'amélioration du quotidien. Pour les enquêtés, l'uniformisation des paysages que renvoie la succession de villages et de demeures identiques le long de l'axe routier n'est pas perçue de manière négative. Au contraire, résider dans un logement construit par l'Etat renvoie à une image positive, celle d'une conception de la modernité pour le moins inattendue aux yeux des partisans de l'idéal perdu du ksar.

\section{TERRITORIALISER PAR LES PRATIQUES ROUTINIÈRES ET QUOTIDIENNES}

Ainsi, alors que la phase d'urbanisation des campagnes s'achève, des indices laissent supposer qu'une urbanité se construit. Elle est perceptible à travers les discours mais c'est essentiellement par le biais des pratiques routinières quotidiennes que progressivement les habitants s'approprient et donnent sens à l'espace produit par l'Etat.

Si l'on traverse les vallées oasiennes du Sahara libyen sans descendre de son véhicule, l'impression d'uniformité domine, similaire à celle qu'évoque Le Clézio à propos d'As Smara, dans le Sahara Occidental. Les commerces de chaque village s'alignent le long de l'axe; on trouve généralement une épicerie, un café, un ou deux vendeurs de cigarettes, autant de primeurs, parfois une station d'essence Libya. Ces quelques boutiques sont fermées durant la journée et accroissent l'impression de non-lieux laissée par ces villages. Elles n'ouvrent que le soir lorsque leurs propriétaires rentrent dans le village après l'emploi de fonctionnaire qu'ils occupent ou des cours qu'ils suivent dans l'un des nombreux centres universitaires dont le Fezzan est doté. Les véhicules qui circulent sur la route klaxonnent, s'arrêtent, leurs occupants descendent, saluent un ami, 
achètent quelques fruits ou légumes et repartent, contribuant à créer un peu d'animation.

Cette animation conduit à souligner un changement majeur du quotidien villageois : la palmeraie, les jardins ou la mosquée ne sont plus (autant) les lieux de sociabilité aujourd'hui. La route s'y substitue car elle émerge non seulement comme le vecteur essentiel de la circulation mais aussi de l'urbanité dans un cadre spatial plus étendu qu'auparavant. En effet, la circulation est omniprésente : on va voir ses amis, on les retrouve dans des lieux à l'écart des villages, parfois dans des sites touristiques. Les jardins, pourtant cités comme l'un des avantages de la localisation dans le Sud, sont peu pratiqués si ce n'est pour les loisirs.

L'analyse des pratiques habitantes confirme de manière plus tangible cette territorialité. Il suffit d'ailleurs de localiser certaines activités pour en avoir une idée. Ainsi, les centres administratifs et commerciaux, ces lieux où l'on va pour la nécessité, contribuent à étendre l'aire des déplacements, tout autant que ceux des loisirs et de l'amitié. Les lieux de loisirs correspondent rarement à des villes, au mieux au domicile, mais le café ne concerne «que les étrangers ou bien si l'on veut voir ensemble une rencontre sportive à la télévision » dit un jeune d'Al Gharayfa. En fait, deux éléments sont recherchés, d'abord l'isolement, jardins, déserts ou bien les sites de baignade tels les lacs d'oasis situé dans l'Erg d'Oubari. Les lieux où l'on va pour voir ses amis recoupent en partie ceux des loisirs mais lorsqu'il s'agit de les localiser, c'est l'ensemble du Wadi qui est représenté. Seule la localisation de la famille diffère puisque toutes les personnes questionnées disent vivre là où vivaient leurs ascendants.

La facilité avec laquelle les déplacements individuels peuvent être effectués donne une tonalité bien particulière aux modalités selon lesquelles les Fezzanais pratiquent leur espace. Elle contribue à créer une proximité spatiale susceptible d'intensifier et de diversifier les liens sociaux. L'accès généralisé aux études supérieures -permis grâce à la multiplication de petits centres universitaires de proximité (6 dans le Fezzan, en dehors de l'université de Sebha)- et la tertiarisation de l'économie contribuent en effet, à l'accroissement des déplacements. En conséquence, le cadre spatial dans lequel se déploie le quotidien s'est singulièrement étendu car tous les habitants peuvent circuler d'un village à l'autre et ainsi accéder à de nouveaux lieux de sociabilité. La dissociation des lieux de la pratique spatiale révèle que le quotidien des Fezzanais s'inscrit dans un espace sélectif et éclaté, selon un jeu d'échelle emboîté : la famille, c'est le village, les amis, la vallée, au-delà s'exprime l'altérité envers les étrangers et les Italiens, nom moqueur donné aux Tripolitains. 
L'impression qui ressort est donc celle d'une diffusion de l'urbanité à l'initiative des habitants, non pas en s'installant dans une agglomération importante mais en intégrant la ville et les pratiques urbaines dans son quotidien. Nombreux sont les habitants qui, par exemple, critiquent les villes du reste de la Libye, notamment celles du Sahara, tout en fondant leur idéal urbain autour de l'idée d'une urbanité sans villes. Nombreux sont, en conséquence, ceux qui ne se considèrent ni comme ruraux alors qu'ils vivent dans l'espace rural, ni comme urbains puisqu'ils évitent tous les tracas supposés de la vie quotidienne dans les grandes villes. Ce positionnement médian est très prégnant dans le discours, ouvertement comme un signe de distinction, voire de fierté mais aussi, de toute évidence, comme un ferment identitaire. Est-ce là l'équation réussie de l'urbanisation saharienne dans les petites agglomérations ?

\section{LES LIMITES D'UNE URBANITÉ IDÉALE}

L'impression laissée en tentant de cerner les contours d'une urbanité saharienne est finalement assez mitigée. Il est nécessaire de nuancer un discours un peu unanimiste à propos d'un « modèle idéal » mais on peut aussi risquer d'évoquer quelques pistes de réflexion.

Au premier regard, l'impression est positive. Le discours majoritaire se construit autour d'une conception de la modernité, dite urbaine, sans les inconvénients de la ville. Dans cette logique, les villes sont suspectées de présenter deux inconvénients majeurs, celui d'accélérer le délitement des structures sociales traditionnelles et celui de promouvoir l'anonymat, «ce signe d'une société toute entière urbanisée » (C. Petonnet, 1994). Ce qui n'est en fait pas du tout avéré comme le révèle le cas de Sebha, la «capitale »du Fezzan, où les stratégies familiales sont extrêmement actives.

Rapidement les limites d'un «modèle idéal » se précisent. L'urbanité est indéniable, certes, mais aussi incomplète. Curieusement, c'est chez un vieil homme que l'on retrouve la phrase la plus amère : «la moindre des choses est plus attirante en ville que dans le village ». On pense alors à tous ces non-dits, tels l'absence d'anonymat et le contrôle social dont pâtissent les jeunes et les travailleurs immigrés. Le regard croisé semble ici peser plus lourd qu'ailleurs, peut-être parce qu'il résulte de deux niveaux de surveillance qui finalement convergent dans le modèle urbain diffusé dans le Sahara libyen. Pour le premier niveau, institutionnel, accentuer l'agglomération des populations, c'est offrir le confort mais aussi contrôler, alors que pour le second niveau, social, l'enjeu vise à la 
perpétuation de la régulation villageoise traditionnelle dans un nouveau cadre spatial. En conséquence, l'urbanisation signifie l'atteinte de la normalité par rapport au reste du pays et la matérialisation d'une intégration nationale achevée. Mais derrière l'unanimité de façade que révèle le discours, on perçoit aussi des décalages entre les attentes de chacun, les limites d'un modèle pour tous.

L'urbanisation des campagnes n'est pas la finalité de la mutation mais une étape majeure dont la particularité est d'avoir été menée par l'Etat. Il est évidemment toujours plus aisé de juger à posteriori mais on sait aussi le risque qu'il y a à porter des jugements trop hâtifs et à considérer que là où l'on ne voit pas un fait, il convient peut-être de chercher les indices d'un processus émergeant ; peut-être que l'urbanité sans ville est l'un d'eux. Dès lors, les constats alarmistes portés sur un processus en cours ont pu induire en erreur, en conduisant à souligner la mort de la ville là où il serait peut-être plus prudent d'évoquer la mort d'une conception de la ville ou en diagnostiquant des pertes d'identité là où de nouveaux référents émergent.

Un des éléments les plus intéressants est l'intense fabrique des lieux publics dans les sociétés sahariennes, telle que la révèle l'étude des pratiques spatiales. On connaît les précautions d'usage liées à l'utilisation de ce terme ; nous entendons ici par lieu public les lieux «public-privé », selon la tripartition proposée par Cornelius Castoriadis ${ }^{23}$, entre l'oïkos (la maison, la famille, la vie privée) et... l'ecclésia, le lieu public-public... où s'exerce... le pouvoir politique ». Ces lieux n'existent pas dans les agglomérations actuelles, où chacun est incité à rester confiné à la sphère privée, et leur production s'effectue selon des modalités bien particulières. En réponse au contrôle social, ces lieux sont généralement créés à l'écart des agglomérations, ils peuvent coïncider avec les palmeraies, devenues pour beaucoup des jardins d'agrément ou tout simplement à un espace suffisamment éloigné que trahissent les traces de pneus hors des pistes, afin de se livrer à des activités prohibées comme la consommation d'alcool. Parfois, ces lieux émergent en coïncidence avec d'autres, notamment ceux du tourisme émergent. On en devine les motivations : symboles de l'ouverture à l'extérieur, ils sont en même temps ceux où l'on peut transgresser les interdits du quotidien sans pour autant avoir à se cacher ; les fêtes d'étudiants, de collègues de bureaux s'y déroulent donc. On notera d'ailleurs, selon une démarche identique, que les jeunes de Koufra privilégient les hôtels internationaux de la ville de Benghazi lorsqu'ils désirent se marier ; elle est pourtant située à près de 2 heures

${ }^{23}$ Cornelius Castoriadis., «L'individu privatisé », Le Monde Diplomatique, Février $1998, n^{\circ} 527$. 
d'avion, soit 1100 kilomètres de l'oasis. Il s'agit de contourner des pratiques sociales du mariage particulièrement onéreuses à Koufra mais aussi de chercher des lieux éloignés, comme c'est le cas dans le Fezzan. 


\section{UNE URBANITÉ, DES URBANITÉS}

Au fil de cette dernière partie, il est apparu que la ville «se fait » à la fois par le haut et par le bas.

Par le haut, elle traduit la vision des aménageurs dont l'objectif était essentiellement de répondre à une urgence et d'accompagner une dynamique d'agglomération faite de communautés. Les velléités de ce modèle se lisent dans le plan d'aménagement de Sebha pour 1988. Dans le reste du Fezzan, la question se posait avec moins d'acuité. En effet, la politique du logement concernait toute la population des villages, donc une seule communauté, ensemble homogène malgré des clivages familiaux encore prégnants. Ce modèle d'aménagement perd de sa justesse, tant au plan fonctionnel comme le prouve les «crises urbaines» qu'au plan humain.

Par le bas, les modalités de l'appropriation de l'habitat et des quartiers témoignent de l'évolution du modèle importé. Elles relèvent en partie du fait de civilisation, qu'il s'agisse de la fermeture des espaces ouverts ou de l'intégration progressive des groupes nomades. Sur ce point, rien ne diffère fondamentalement des constatations effectuées dans le reste du Sahara maghrébin. Plus discrets, mais tout aussi fondamentaux, les échanges, entre personnes, de produits, se développent, contribuent à l'émergence de nouvelles centralités et donc à de multiples opportunités d'interactions sociales.

En somme, pour reprendre les termes de la définition de Jacques Lévy (1994) sur l'urbanité, la densification s'accompagne d'une diversification croissante. Mais la diversité suscite-t-elle pour autant le passage de l'agglomération à la ville ? Reprenons le rapport au monde énoncé plus haut. La maison est dans le quartier, le quartier dans la ville mais la ville est plus que l'addition des quartiers car elle fait système. Or elle ne peut faire système que dans l'interaction et si la diversité sociale s'accompagne du cloisonnement spatial, le modèle se limite à celui de l'agglomération.

Cette idée nous semble fondamentale dans le Sahara libyen, et sans doute même dans l'ensemble du Sahara car elle pose problème. Elle conduit à repenser la question du lien entre la communauté et l'agglomération. La relation entre ces deux entités, l'une sociale, l'autre spatiale, a été abordée à plusieurs reprises. Le fait d'évoquer Sebha et le réseau du Wadi Al Haya incite à pousser un peu plus avant la réflexion, dans deux cadres géographiques différents. 
A Sebha, l'effet de taille contribue, dans un premier temps, à créer l'altérité et donc à ce que les personnes privilégient le lien familial aux interactions sociales avec l'extérieur. L'installation dans l'agglomération étant collective, le quartier est le niveau le plus pertinent afin d'appréhender le marquage spatial de cette altérité. Pour autant, la dynamique initiale ne doit pas conduire à sous-estimer les petits signes de «la ville en train de se faire». On les repère à travers les nouvelles centralités et les ambiances qui s'en dégagent. On repense à cet esprit des lieux évoqué en introduction auquel contribuent de manière sensible la présence d'immigrés. Pour autant, ces lieux de sociabilité ne concernent pas tous les habitants. Nous parlons ici des hommes, car ce sont eux qui participent à la sociabilité citadine. Les femmes restent cantonnées à celle des espaces privés ou semi-privés, maison et quartier. Le niveau de la ville, qui correspond à celui de l'interaction hors du cercle familial et communautaire est masculin, si l'on excepte ce qui concerne l'emploi.

Dans le Wadi, le fait que la communauté et la ville ne fassent qu'un en restreint tout autant la diversité. Celle-ci n'est perceptible qu'en prenant un peu de hauteur, c'est-à-dire en abandonnant définitivement l'idée du village cellule autarcique pour le penser dans son environnement social et spatial, celui de la vallée. On perçoit alors mieux à quel point, ici, l'étroite association entre la circulation et les interactions sociales contribue à l'élargissement de l'aire spatiale des pratiques. Or en multipliant ces pratiques par le travail, les études ou bien les loisirs, les personnes multiplient les lieux faisant partie de leur vécu, créant ainsi la diversité. Trivialement, nous pourrions résumer cette idée en disant que la voiture repousse les limites de l'altérité entre l'ici et l'ailleurs.

Finalement, à Sebha, la ville se dessine ou plutôt, est redessinée par ses habitants de la maison à la ville en passant par le quartier. Dans le Wadi Al Haya, on assiste plutôt à une recomposition régionale multi-scalaire appuyée sur des représentations spécifiques de l'urbanité. Elles s'expriment à la fois dans les pratiques et le discours. Partout la ville se fait mais autrement. Peut-être est-ce là la spécificité d'une hypothétique urbanité saharienne. 


\section{CONCLUSION GÉNÉRALE : QU'EST-CE QUE L'URBAIN DANS LE SAHARA LIBYEN ?}

Pour aller vite, et en usant de la rhétorique grammaticale, disons que nous avons tenté, au long de ce travail, de décliner un concept flou, l'urbain, à divers modes et divers temps. Ceci nous a conduit à envisager l'évidence, celle d'un processus d'urbanisation rapide et généralisé mais aussi celle du devenir d'une société dans un cadre spatial très largement modifié. Présentée de la sorte, cette entrée parait doublement banale, parce qu'elle est le fondement d'un vaste champ de la géographie sociale et que l'on ne manque pas de travaux de qualité mettant en valeur les interactions entre dynamiques urbaines et changements sociaux dans le Sahara contemporain.

Quel intérêt présente donc un travail de ce type sur le Fezzan ? Certes, il s'agit d'une région vaste, excentrée, sise en Libye et pour toutes ces raisons peu connues ; mais le géographe sait que les terrae incognita ne manquent pas et cela ne suffit pas toujours à rendre le choix d'un terrain pertinent. En revanche, le Fezzan a subi, à l'initiative de l'Etat, des mutations socio-spatiales d'une telle ampleur qu'il y avait sans doute là matière à interroger les ressorts du changement à l'échelle du Sahara maghrébin mais aussi de tout le Sahara.

Deux objectifs ont sous-tendu ce travail. D'une part, la nécessité de construire une grille de lecture des mutations urbaines du Sahara, fondée dans un premier temps sur des lectures concernant essentiellement le Sahara maghrébin puis en enrichissant ou en nuançant ce corpus à l'épreuve d'un terrain. D'autre part, la volonté de resituer l'étude dans la dialectique des relations entre le social et le spatial à un moment charnière : celui où la phase d'urbanisation volontariste ralentit et où des politiques d'ouverture et de libéralisation signalent le désengagement des Etats.

Pourquoi ce choix ? Le Sahara a connu depuis quelques décennies une urbanisation extrêmement rapide menée par des Etats. Or ces Etats en aménageant les déserts se sont inscrits en droite ligne dans une modalité qui veut que ce qui s'y passe soit toujours décidé à l'extérieur, par des commerçants, on a alors parlé de relais, par des colonisateurs, on a plutôt parlé d'hinterland, par des Etats, on parle alors de périphérie plus ou moins intégrée ou en voie de l'être. Ce fil directeur est lourd de sens car il m'a conduit à supposer que cette urbanisation s'est faite sans ou contre les sociétés. Dans le contexte de désengagement actuel se posait la question de 
comprendre comment cette urbanisation avait été vécue, et dans quelle mesure elle donnait lieu à des réappropriations. En effet, quoique l'on puisse penser de cette dynamique urbaine qui est dynamique de fond puisque touchant le monde entier, on se sent bien obligé d'en prendre acte ; reste alors à déterminer quels sont ses contours là où on l'observe.

Ce questionnement sous-tend le travail mené dans le dernier chapitre, visant à confirmer la rupture entre un urbanisme imposé, symbole de modernisation subie et les aspirations des habitants. C'est pourtant l'inverse qu'ont révélé les réponses (Ch.9), non seulement, cet urbanisme n'était pas rejeté mais il était signe de distinction et faisait déjà partie des représentations de l'espace. Ce constat n'a finalement rien pour surprendre car de dix à quinze années sont passées depuis cette phase d'aménagement volontariste et en conséquence, une partie importante de la population, les plus jeunes, n'avaient connu que cela. Après tout pourquoi auraient-ils idéalisé les ruines des ksour, transformées souvent en décharges publiques ou enclos à bétail ? A démarche artificielle réponses décalées ; c'est finalement dans la pratique du quotidien, fondée sur l'attente, les salutations, les fêtes, la circulation d'un village à l'autre que sont venues quelques clés de compréhension sur ce que signifie l'urbain dans le Fezzan.

Le fait de travailler sur un pays peu connu, à propos duquel les ouvrages les plus récents ont désormais plus de dix ans, incite à décrire un espace tel qu'il se présentait quitte à confirmer que ce que l'on y observe ne diffère guère de ce que d'autres ont observé ailleurs. C'est vrai de l'action de l'Etat (ch. 2) à propos de laquelle le constat de Jean Bisson (1993) qui notait que si les politiques variaient, les effets spatiaux étaient néanmoins identiques dans tout le Sahara maghrébin garde toute sa pertinence ; c'est vrai aussi des transformations des tissus urbains (ch. 5) et des complémentarités entre dynamiques urbaines et rurales (ch.6). En fin de compte, le parti pris ici a consisté à ne pas évacuer ce qu'il y avait de banal en Libye, car si l'on doit dire ce qui est différent, on peut aussi dire ce qui est semblable.

Les questions renvoyées par les fezzanais ont fondamentalement contribué à guider la présente recherche : les problèmes de l'eau dans les villes, des ordures, de la gestion de ville -qui ressemblent à beaucoup d'autres mais en tout cas pas aux plans d'aménagement que l'on continue à en dresser (ch.7)- mais aussi aux multiples discours sur l'espace tenus à propos des villages et de Sebha qui incitent dans un premier temps à aborder cette dernière comme l'antithèse des villages puis finalement comme une autre manière de vivre ensemble, de produire de la société 
(ch.8). En définitive, une définition de l'urbanité à contre-pied se dessine qui peut se fonder sur la densité et la diversité mais aussi sur différentes manières de considérer la proximité, notamment en la créant comme substitut à l'absence de densité, par la mobilité. Peut-être y a-t-il là un champ de réflexion conceptuel fondé sur la compréhension du changement social dans un contexte spatial déterminé.

Autre conséquence de l'observation, la rencontre avec un cosmopolitisme inattendu dans ces «confins ». Le Sahara n'est donc pas périphérique ? Il serait même largement ouvert ? Ces questions élémentaires émergent et trouvent réponses à l'observation de la vivacité des échanges dans le Sud libyen (ch. 3). De là en ont découlé d'autres : observe-t-on ici les limites de l'intégration nationale ? La présence de deux logiques qui s'opposent tout en se complétant ? L'interprétation des croissances urbaines renvoie à cette dernière question puisqu'elle coüncide globalement avec la phase d'achèvement du processus d'intégration nationale mais qu'elle soulève aussi la question du statut des marges dans la remise en cause des cadres nationaux.

La question des acteurs (ch.6) apparaît comme la plus frustrante dans la recherche de réponses. D'où est venue cette difficulté qui fait que malgré un certain nombre de monographies, d'itinéraires personnels, la dimension politique, portant sur les relations entre le national et le local, soit si difficile à aborder? Il y a d'abord cette absence de cadre conceptuel depuis des travaux tels ceux de John Davis (1987) qui conduit au fait que depuis les années 1970, on ne trouve plus réellement d'étude de terrain permettant de disposer de quelques clés d'interprétation. Par conséquent, les effets d'une coupure prolongée avec une société libyenne qui a connu des mutations considérables en 20 ans ne sont pas prêts d'être comblés. On peut aussi se réfugier derrière le constat établi par Dirk Vandewalle (1995, 1998) qui considère que les autorités jamahiryiennes se sont livrées à un processus de dépolitisation sur le long terme de la population libyenne. En revanche, si les rouages de la politique sont monopolisés, on sait que l'investissement du politique s'effectue dans toute société. On prend donc la mesure des chantiers qui s'ouvrent.

On retiendra trois pistes, portant sur les relations entretenues entre le « haut », le pouvoir central, et le « bas », le local, les temps de ce territoire saharien et la lecture d'un espace totalement urbanisé. 


\section{«HAUT » ET « BAS », NATIONAL ET LOCAL, DES RUPTURES À NUANCER}

Au bout du compte, où a mené le processus d'urbanisation impulsé avec vigueur durant deux décennies ? Il a d'abord été une réponse, en termes quantitatifs, à la croissance démographique qu'a connue le Sahara libyen. Il fallait loger vite et mieux, ce à quoi s'est employé l'Etat, acteur clé, mais aussi donner aux populations des motivations pour rester là où elles vivaient, plutôt que de quitter définitivement les espaces oasiens qui traversaient une crise grave et durable. La diffusion de l'urbain, l'extension des réseaux de transport et l'évolution du maillage administratif sont quelques-uns des éléments fondamentaux de toutes les politiques d'intégration nationale. La force du projet social se manifeste de manière tangible dans l'intervention étatique libyenne car l'urbanisation n'est en fin de compte qu'un élément d'une politique plus ambitieuse, celle de la redistribution d'une partie de la rente, aux mains de l'Etat, à la population libyenne. Par conséquent, l'urbanisation est aussi une manifestation de l'indispensable transaction qui lie les autorités aux sociétés qu'elles gouvernent.

De l'approche spatiale de la dynamique urbaine, on glisse progressivement vers d'autres questions, telle celle de la légitimité des actions engagées. Après tout, particulièrement dans les plus petits centres, l'urbanisation est pour le moins un processus brutal, fondé au plan spatial sur la politique de la table rase, comme on peut le voir en analysant les tissus urbains du début des années 1980. Smati Ababsa (1997) rappelle crûment, sous forme de réquisitoire à l'encontre des décideurs algériens de l'aménagement saharien, des éléments qui incitent à prendre du recul par rapport à l'objet d'étude et à ne pas oublier certains faits. «Si l'oasis est une réalité pour ceux qui y vivent, c'est-à-dire là où ils ont élu domicile, où ils élèvent leurs enfants, où ils rêvent de leur avenir et où ils triment au quotidien pour réaliser qu'une infime partie de leurs rêves, que peut-elle bien être pour ceux qui décident... qu'une oasis doit être développée d'une manière et pas d'une autre ?... Ils sont toujours de passage là où ils s'arrêtent. Entre deux mutations, ils vivent leur affectation au Sahara comme un incident de parcours... Lorsqu'on décide de construire des cités-dortoirs que ne se pose-t-on pas la question de savoir de quoi vont vivre leurs nouveaux résidents ? ». Certes, cet argumentaire renvoie à une situation nationale spécifique, celle de l'Algérie, où l'intervention étatique dans le Sahara s'est matérialisée par l'émergence de la figure du «nordiste », fonctionnaire peu au fait et peu intéressé par les lieux où il est muté, et par conséquent responsable de décisions calamiteuses dans le champ de l'aménagement. Mais on devine une critique adressée à tous les 
Etats, suspectés, par leur traitement spatial de la portion du Sahara sur laquelle ils exercent leur autorité, celle d'avoir diffusé, autoritairement, un modèle urbain inadéquat, uniformisant et porteurs de tous les maux urbains et sociaux.

Pourtant, il semble d'emblée nécessaire de nuancer l'image par trop manichéenne d'une urbanisation imposée contre la société et de se questionner sur la pertinence du modèle. Ne reflète-t-il pas plutôt les dynamiques de la période à laquelle il a été conçu ? Il n'était d'évidence pas à même d'anticiper les bouleversements qu'a connu la composition de la société saharienne. Comment la rupture entre les visions du haut et les aspirations du bas s'est-elle traduite en Libye? L'Etat a répondu, globalement, en termes de recomposition spatiale à une demande sociale comme ailleurs mais selon des spécificités qu'il ne faut pas négliger. Ainsi, il n'a jamais été question de déstructurer le mode de fonctionnement de la société, que l'on résume par le terme de tribalisme. Le modèle urbain étatique, modèle du village, de la communauté sociale élémentaire, qu'il nous a semblé percevoir jusque dans les plus importantes agglomérations, n'était en somme qu'un avatar de la formule du changement dans la continuité. Il ne faudrait pas voir là une solution conservatrice visant à freiner un changement inéluctable mais plutôt considérer cette option comme un élément du consensus permettant l'adéquation entre une production spatiale impulsée par le haut et une société qui subissait le mouvement. Ainsi, en Libye, le face-à-face entre les autorités nationales et les sociétés locales s'est longtemps exprimé avec force par le biais du processus d'urbanisation. Dans le reste du Sahara, le clivage était surtout local, mettant face-à-face la communauté (jamaa) et l'individu. L'Etat n'était dans ce rapport souvent qu'un acteur à posteriori, institutionnalisant des dynamiques internes aux villages.

Le face-à-face entre acteurs du national et acteurs du local perd aujourd'hui de sa force, du moins sous la forme qu'on lui connaissait jusqu'à présent. On a vu que le désengagement de l'Etat en est la cause essentielle, qui apparaît jusque dans la gestion urbaine. Certes, on peut arguer du fait que l'ampleur de l'urbanisation était au-dessus de ses moyens, comme le fait notamment Saad Qezeiri (1986) et donc convenir que la chute des prix du baril de pétrole entraîneraient de manière mécanique le recul de l'interventionnisme étatique. Mais rétrospectivement, ce désengagement n'apparaît que comme le déclencheur d'un nouveau mode de relations entre l'Etat et les communautés locales. En effet, ni l'une ni l'autre de ces deux parties n'est restée statique durant ces trois dernières décennies. L' «Etat sans Etat » libyen a délaissé, voire supprimé, quelques-uns des outils à même 
d'assurer le projet politique et social de son «Guide» Mouammar Kadhafi. Cet acteur très actif sur la scène internationale a progressivement pris acte de sa marginalisation politique durant le temps de l'embargo et tenté d'élaborer de nouveaux projets, tel l'Union africaine, où une fois encore géopolitique interne et externe sont étroitement liés. La société fezzanaise n'est plus non plus la même, nourrie par la croissance démographique, la fin de l'exode rural, les flux migratoires inter-arabes puis africains, elle présente aujourd'hui un visage bien différent de celui qu'elle donnait à voir en 1969.

Toutefois, ne nous y trompons pas, le mouvement du haut vers le bas, mené par l'Etat, façonne l'espace et par ce biais la société. Le mouvement inverse, du bas vers le haut n'est en fait qu'adaptation, appropriation, contournement, certainement pas remise en cause du pouvoir central. Pour autant, dans les interstices du processus d'urbanisation étatique se lisent, se pressentent parfois, des bribes du changement social dans le Sahara central. Il se traduit par les actives recompositions qui traversent la société fezzanaise, plus ouvertement hiérarchisée, plus nettement sur les critères financiers.

La complexité des stratégies à l'œuvre et la redéfinition des fondements des relations de clientèle sont partout flagrantes. Les notables, désormais intégrés dans des réseaux politiques ou économiques nationaux, affirment leur place d'élites locales et surtout de relais vers l'extérieur. Leurs stratégies, multiformes, se développent au plan local, à l'échelle des villages, des vallées et pour certains du territoire national. La plupart des habitants, contraints de s'adapter afin de pallier les carences liées au désengagement de l'Etat, s'intègrent au contexte de l'urbanisation en cours par des stratégies individuelles d'acquisition ou d'amélioration de l'habitat. Pour autant, il convient de ne pas restituer une vision trop manichéenne de la réalité. En effet, ce désengagement, après une période de redistribution qui a certes profité au plus grand nombre mais en limitant tout autant les marges de manœuvre des populations, permet aussi à la société libyenne d'opérer une réappropriation spatiale, dans le cadre des structures familiales, par le biais d'une gestion locale et coutumière du foncier.

Un mouvement impulsé de l'extérieur ne le reste pas ou alors, au Sahara, il disparait. Cette urbanisation reflète une vision idéologique de l'espace fondée sur un traitement égalitaire du territoire et des habitants. Elle n'est pourtant pas un épiphénomène sans lendemain que l'on ne pourrait décliner qu'au négatif. Les dynamiques d'extension et de diversification du tissu urbain prouvent que la fragmentation urbaine ne 
doit donc pas être interprétée comme une crise de la ville. Bien au contraire, elle confirme à quel point l'urbain, au-delà des chiffres qui permettent de constater l'agglomération continue de la population, est au cœur de la recomposition spatiale du Fezzan. Ici, cette idée prend toute sa dimension puisque l'espace rural se transforme sous l'impulsion de ceux qui vivent dans les villes. En définitive, la relation entre l'Etat et les tribus semble remarquablement s'adapter à la rapidité des changements politiques, économiques et sociaux, décrédibilisant tout discours passéiste sur son mode de fonctionnement.

\section{LE TEMPS LONG DU RÉSEAU ET LE TEMPS COURT DU TERRITOIRE D'ETAT}

D'emblée, il est nécessaire de revenir sur les limites des aires dans lesquelles s'inscrivent la plupart des études portant sur le Sahara. S'il y a un Sahara maghrébin et un Sahara sahélien, de nombreux indices incitent maintenant à considérer qu'il y a aussi un Sahara. Celui-ci se caractérise par des liens tissés au-delà des frontières par les migrations, les échanges marchands et les liens tribaux.

On ne peut réduire l'urbanisation saharienne, particulièrement en Libye, à l'expression de l'intégration nationale. Certes, l'Etat, en développant les infrastructures ou les services administratifs et plus généralement en ancrant le Sahara au territoire national a impulsé le processus, peut-être avec plus de force dans les régions oasiennes et agricoles qu'ailleurs. De plus, le Sahara est vaste, même si l'on n'envisage que des «portions » à l'intérieur de pays comme la Libye ou l'Algérie, et les dynamiques des marges septentrionales n'y sont pas du même type que celles des régions méridionales; elles ne relèvent pas des mêmes ressorts. L'intégration progressive au territoire national des premières contraste avec celle, plus tardive mais aussi incomplète des secondes, plus lointaines, laissant supposer que l'urbanisation des marges du territoire libyen marquent l'achèvement d'un processus d'intégration nationale.

Au plan géopolitique émerge une nouvelle donne saharienne qui remet en cause cette lecture du processus impulsé du centre vers la périphérie du territoire national. La politique africaine de la Libye constitue actuellement l'élément le plus voyant d'intégrations régionales institutionnelles étendues aux régions sahariennes. Mais c'est discrètement que se construisent des intégrations régionales «par le bas », silencieusement mais de manière plus dynamique que celle à laquelle peuvent aboutir les Etats. Elle s'effectue à l'instigation des gouvernants qui bénéficient de 
leurs réseaux utilisés à titre privé, mais aussi de tous ceux qui circulent à la recherche d'un emploi, car ils fuient des espaces de crises ou d'opportunités à saisir.

Ainsi, les foyers de peuplement les plus méridionaux du territoire national sont au cœur d'intenses circulations et intégrés dans un espace relationnel dense et transnational. Cet espace, produit par des nomades Touaregs et Toubous et des familles de commerçants arabes dispersées entre différents Etats, se construit dans la proximité qui permet circulations et d'échanges constants mais aussi dans la durée car les réseaux marchands et les alliances matrimoniales le pérennise. Les troubles géopolitiques, qu'il s'agisse de guerres, de rébellions ou de jeux d'influences entre Etats voisins, contribuent à l'alternance des périodes de cloisonnement et d'ouverture. L'ouverture, comme on peut le constater actuellement, se concrétise par l'intégration de ces espaces sahariens à des réseaux relationnels plus vastes, associant sur les mêmes itinéraires la circulation de proximité qui implique les régions frontalières du Sahara libyen et celle plus lointaine avec l'Afrique de l'ouest. Si les premières se maintiennent avec plus ou moins d'intensité selon les relations entre Etats, les secondes dépendent plus étroitement d'une conjoncture politique changeante.

\section{LA PORTÉE D’UN FAIT SPATIAL : L'URBANISATION GÉNÉRALISÉE}

En opérant un glissement de l'attention portée aux mouvements qui vont du haut vers le bas à ceux qui partent du bas, on constate que des urbanités émergent qu'il est nécessaire d'explorer.

L'urbanité du Fezzan se distingue des modèles théorisés ailleurs, généralement dans les pays développés. En effet, les secondes s'inscrivent dans une histoire urbaine qui n'a pas dans le Sahara d'équivalent, où elle est plus durablement inscrite dans les représentations que dans les espaces. Ce n'est en conséquence pas à l'initiative des citadins ni des villes que part le mouvement, comme permettait de le pointer l'analyse des représentations et du discours sur l'urbanité dans le Wadi Al Haya. Les déclencheurs ici sont d'une part l'Etat et de l'autre une configuration spatiale particulière (même si elle correspond à un tiers des surfaces émergées du globe), les espaces arides. Pourtant, l'urbanité du Fezzan pointe le devenir de bien d'autres urbanités, fondée sur les faibles densités et la dispersion des populations et par conséquent la nécessité d'intégrer le couple distance-proximité, le dépassement du clivage villes-campagnes.

A l'échelle locale, intra-urbaine ou villageoise, l'urbanité se manifeste par la réappropriation du modèle étatique, individuelle à travers la 
transformation du logement notamment, ou collectivement. Il apparait alors que l'agglomération devient ville sous l'impulsion de ceux qui y vivent car ils la pratiquent, la transforment, la signifient, et y inventent leurs propres modèles par une intense fabrique sociale. La fragmentation du tissu urbain donne une première piste, même si elle reste encore limitée compte tenu de la force des liens communautaires. L'exemple des « quartiers mixtes » rappelle que malgré les tiraillements induits par les hiérarchisations sociales, le lien familial prime encore dans de nombreux cas comme facteur de localisation dans les villes. Ces effets contraires indiquent une nouvelle piste de réflexion des dynamiques urbaines. Les recompositions spatiales relèvent à la fois de la segmentation, logique de regroupement à fondement tribal, et de la fragmentation, facteur de partition fondé sur les inégalités de revenus mais aussi sur l'altérité vis-à-vis de « l'autre », notamment l'étranger.

Sur ce point, la diversité croissante des populations constitue un élément fondamental. Elle entraîne évidemment de la diversité dans les agglomérations. Toutefois cette diversité ne devient opératoire comme facteur sociétal que lorsqu'elle contribue au décloisonnement. Dans un premier temps, la juxtaposition des groupes par origine, villageoise pour les Libyens, nationale pour les immigrés, domine, repliés sur leur communauté, qui s'approprient des portions de l'agglomération. Par conséquent, elle ne s'accompagne pas, momentanément, de cette dynamique de mixité sociale. Pour cela, il faut du temps et l'on n'en perçoit au mieux que des frémissements.

Dans une certaine mesure, l'urbanité émerge donc dans l'ensemble du Fezzan. C'est à Sebha que l'on appréhende le mieux ses contours. L'importance numérique des immigrés et des réfugiés a contribué à l'émergence de centralités fondamentales à l'insertion dans la ville des nouveaux arrivants. En effet, en évoquant le réseau saharien, on pense au rôle de carrefour et donc de transit du Fezzan mais il ne faut pas pour autant négliger les capacités de rétention de cette région. Or sa croissance démographique s'appuie très largement sur l'apport et l'installation de populations étrangères ; les centralités qu'elles contribuent à produire sont porteuses d'une neutralité certaine vis-à-vis des clivages communautaires entre Libyens. Il n'est alors pas improbable qu'elles suscitent l'émergence de ces lieux publics que tout semble empêcher, planification urbaine, poids du groupe, primat de l'automobile sur tout autre mode de déplacement.

L'urbanité ne peut être considérée uniquement comme une clé de lecture des dynamiques socio-spatiales à l'échelle la plus fine. Elle s'exprime aussi à l'échelle régionale comme un puissant moteur du 
mouvement de territorialisation de la société. Comment se dessine l'urbanité à l'échelle d'une région aussi vaste ? La circulation joue un rôle ambigu dans l'émergence de l'urbanité puisque ses modalités diffèrent selon la taille de la ville. Convenons que la motorisation a très largement contribué à façonner la morphologie des espaces urbains mais aussi à modifier le rapport des citadins à ces espaces, permettant souvent la mise à l'écart voulue, le retranchement du reste de la société (J. Lévy, 1999) mais nuançons cette proposition dans le contexte du Fezzan. Dans la walking-city d'avant la Révolution Industrielle, en Europe, les possibilités d'interactions sociales étaient sans doute plus importantes que dans les métropoles contemporaines. Les transports en commun, puis individuels, en favorisant l'extension du tissu urbain, ont contribué à un cloisonnement croissant. On ne peut s'empêcher d'effectuer un parallèle avec Sebha, modèle de l'agglomération. L'utilisation à outrance de l'automobile n'illustre-t-elle pas la profonde privatisation de l'espace qui la caractérise ? La relation entre l'organisation en quartiers communautaires et le modèle routier est tentante.

Ce principe perd de sa pertinence dans le cas des plus petites agglomérations. Sans circulation, les villages, malgré leur nombre et les faibles distances qui les séparent, ne seraient que des «quartiers» juxtaposés mais aussi cloisonnés le long des axes routiers. L'importance de la motorisation facilite les échanges. Elle contribue à créer une proximité spatiale susceptible d'intensifier et de diversifier les liens sociaux. Nous avons vu que les représentations des populations intègrent cette idée de points, les villages, reliés par une ligne, l'axe routier. L'image d'une région enracinée, entrée commode pour aborder les espaces de fortes densités du Sahara, perd donc de sa validité. Nous constatons la fluidité croissante de ces limites, entre particularisme de la vallée et intégration à une région articulée autour du centre principal, Sebha.

L'étude des pratiques révèle que l'on assiste à une véritable fabrique de lieux publics, souvent à l'écart, parfois en coïncidence avec d'autres lieux, ceux du tourisme émergent. Les «métriques pédestres » contribuent sans doute à un idéal de ville, favorisant le maximum d'interactions. Les «métriques automobiles »n'en sont pas moins, dans le cas des petites agglomérations, un substitut à la proximité, favorisant l'ouverture. On peut sans doute trouver dans cette thématique une manière de repenser le rôle des infrastructures routières dans le Sahara contemporain. Considérées comme l'élément essentiel du désenclavement et de l'intégration nationale, perçues en conséquence, souvent de manière négative, comme le véhicule de la dynamique d'urbanisation/modernisation des campagnes, elles 
deviennent aussi l'instrument par lequel les populations s'approprient l'espace et créent leur urbanité.

Nous disions en introduction que l'une de nos principales préoccupations dans ce travail était de penser l'après-Etat au Sahara. L'exercice est fondamental et le choix du Fezzan ne pouvait que faciliter cette démarche. On peut d'abord énumérer les intérêts et les limites du cas libyen. La Libye se différencie au moins sur deux plans de ses voisins, d'abord par l'importance des moyens financiers issus de la manne des hydrocarbures qui ont permis aux autorités politiques de mettre en œuvre une politique d'aménagement ambitieuse mais peu soucieuse des ressources naturelles, notamment en eau ; ensuite parce que la Libye est un pays d'immigration, contrastant en cela avec l'ensemble des Etats voisins. Contre-exemple évident, le Sahara libyen est aussi un observatoire privilégié de logiques partout initiées mais rarement menées à leur terme. L'analyse de ces logiques dans un contexte décalé permet en retour d'enrichir une grille de lecture afin de comprendre d'autres régions sahariennes selon de nouvelles perspectives.

En nous positionnant loin des marges septentrionales du Sahara, nous nous éloignions aussi des effets les plus marquants de ce gradient nord-sud qui conduit à généraliser à l'ensemble du Sahara des dynamiques qui touchent ses marges, mieux connues car mieux étudiées. Il n'est pas question de considérer les dynamiques actuelles comme l'amorce d'une nouvelle table rase, identique à celle qui a profondément modifié le Sahara libyen durant les années 1970 et 1980. Au contraire, nous avons tenté d'être attentif aux écarts, aux débordements, aux appropriations qui contribuent à l'évolution de ce modèle.

Enjeu national et international de premier plan, le Sahara est longtemps apparu au Maghreb comme l'espace rêvé des projections d'acteurs qui en sont extérieurs, militaires, gouvernants en quête de légitimité ou de projets fédérateurs à proposer. Loin de ces événements médiatisés où l'imaginaire a souvent pris le pas sur le réel, il est un espace vécu et en mutation constante. Le Sahara est aussi une «ville» pleinement intégrée aux dynamiques du monde actuel. 


\section{TABLE DES ILLUSTRATIONS}

Figure 1 : Urbanisation et circulations : une approche diachronique ............ 10

Figure $2:$ La structuration spatiale du Sahara ..........................................26

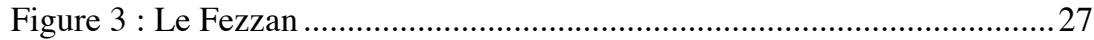

Figure 4 : Le désenclavement du Sahara libyen ............................................ 35

Figure 5 : Les agglomérations du Sahara libyen en 1973 ............................40

Figure 6 : Les agglomérations du Sahara libyen en 1995 ...........................4 41

Figure 7 : Les migrations internes en Libye de 1973 à 1995 ........................45

Figure 8 : Le carrefour migratoire libyen .............................................57

Figure 9 : Oubari, la morphologie urbaine (1981-2000) .............................92

Figure 10 : Les dynamiques de l'agriculture dans le Fezzan ........................99

Figure 11 : Oubari, un périmètre agricole étatique en crise ........................ 103

Figure 12: L'agriculture périurbaine autour de Sebha................................ 104

Figure 13 : Sebha, extension et migrations (1966-2000) ............................ 129

Figure 14 : Sebha en 2000, la morphologie urbaine ................................. 136

Figure 15 : Quatre visions du Fezzan ................................................... 142

\section{TABLE DES TABLEAUX}

Tableau 1 : Les villes sahariennes de plus de 10000 habitants (mi-1990)...... 4

Tableau 2 : Nombre d'agglomérations par strate (1973-1984-1995) .............38

Tableau 3 : Mesures de l'urbanisation dans le Sahara libyen .........................39

Tableau 4 : Les types d'habitats, essai de mesure .........................................86

Tableau 5 : Taux de croissance du bâti entre 1981 et 2000 (en \% par an).....86

Tableau 6 : Trajectoires urbaines................................................................. 88

\section{TABLE DES PHOTOGRAPHIES}

Photographie 1 : Camion à destination du Niger (Sebha) ............................51

Photographie 2: Takarkiba, le modèle urbain étatique ................................90

Photographie 3 : « Lac » d'eau stagnante à Oubari ...................................... 118

Photographie 4 : Transformation des logements collectifs public à $\mathrm{Al}$ Gharayfa

120

Photographie 5 : Autoconstruction à Al Gharayfa ..................................... 121

Photographie 6 : Locatif pour migrants à Sebha ......................................... 122

Photographie 7 : Squat Toubou dans les ruines du ksar d'Al Hajara (Sebha)123

Photographie 8 : Nomades et réfugiés tchadiens en périphérie de Sebha.... 124

Photographie 9 : Cloisonnement des balcons à Sebha.................................. 125

Photographie 10 : La tente de mariage, une appropriation temporaire de la rue133 


\section{INDEX DES NOMS DE LIEUX}

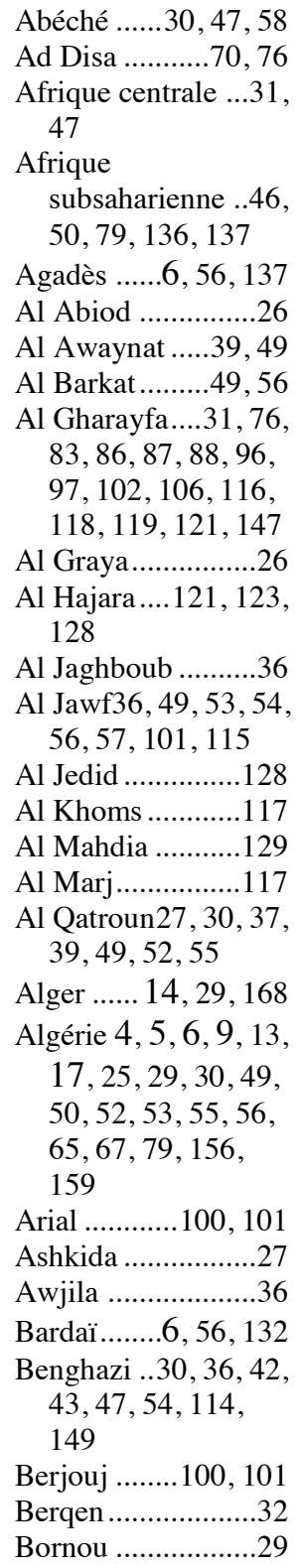

Brak....31, 32, 37, 43, $96,98,105,106$

Dépression de

Mourzouq...27, 28, $32,39,56,97$

désert Libyque.......25

Dirkou .....................52

Djanet .....................52

Edri..................27, 32

Egypte .31,46, 51, 53

El Oued ..........53, 140

Erg d'Oubari ..26, 74, 147

Europe 25, 46, 47, 52, 162

Faya ...........47, 53, 56

Fjej ............26, 74, 98

France..12, 29, 32, 47

Gabraoun.74, 75, 141

Germa.83, 86, 87, 88, 89

Ghadamès.30, 36, 39 , $43,50,60,64,78$, $79,80,81,82,122$, 142

Ghat....30, 31, 36, 37, $42,50,52,56,67$, $68,69,95,96,142$

Hajara ....................129

Houn .........................36

Irawan ..........100, 101

Jalou ................39, 114

Jebel Nefousa .........27

Joufra......................39

Kanem ................47, 48

Kawar ...............25, 52

Khartoum .........54, 58

Koufra 13, 25, 36, 39, $48,49,57,101$ 115,149

Maghreb .3, 4, 7, 17, $25,44,50,53,64$, $91,126,140,163$

Mahrouqa ................32
Maknoussa49, 98, 99, 100, 101

Mali.4, 5, 17, 49, 51, $52,55,71,131$

Maroc ....4, 5, 13, 17, $25,30,39,51,52$, 140

Mauritanie ...4, 5, 17, 42

Mizda ..... 39

Mourzouq 15, 26, 28, $29,30,31,37,42$, $49,56,60,67,68$, $96,98,129$

N'Djamena .. 58, 132, 137

Nalout.............. 39, 81

Niamey ............ 54, 58

Niger... 4, 5, 6, 9, 17, $46,49,51,53,54$, $55,56,58,71,106$, $131,137,142$

Nigéria............ 51, 53 Oubari.26, 32, 37, 43, 49, 50, 52, 56, 67, $70,83,86,87,88$, $89,92,93,97,98$, $100,101,103,105$, $117,118,132,141$, 142

Oum al Aranib ...... 98

Qourda........ 128, 129

Sahara central 1, 4, 6, 14, 17, 28, 34, 46, $50,58,89,158$

Sahara français 12, 15

Sahara libyen .....3, 9, 19, 20, 30, 36, 38, $39,40,42,43,46$, $48,50,58,60,68$, $80,81,97,100$, $101,103,110,116$, $120,127,139,142$, 
$145,146,148,151$, $153,156,160,163$ Sahara maghrébin.. 5, $7,9,13,16,39$, $67,112,141,151$, $153,154,159$

Sahel4, 5, 16, 17, 27, $29,30,49,60,131$, 137,141

Sebha....6, 13, 27, 31, $36,37,41,43,44$, $47,49,50,51,52$, $53,54,55,56,58$ $61,68,69,71,74$, $76,83,84,86,87$, $88,89,95,96,97$, 101, 102, 104, 105, $106,112,117,118$, $119,121,123,124$, $125,128,129,130$, $131,132,134,135$, $136,137,138,142$,
$144,147,148,151$, $152,154,161,162$

Sirte ..........................

Soudan 51, 53, 54, 55 . 58

Souf ...............15, 140

Soukna.....................30

Tahala....98, 100, 105

Takarkiba $.87,88,89$, $90,91,93,119$

Tamanghasset.. 6, 13, $50,52,56$

Tchad.. 4, 5, 6, 9, 17 , 29, 46, 48, 49, 51, 53, 54, 55, 56, 58, 93, 106, 115, 131, 132,137

Ténéré ..............25, 52

Tibesti $6,48,49,132$

Tmessa .................100

Tozeur .......... 15, 122

Traghen ..................28
Tripoli.. 6, 29, 30, 36, $37,42,43,47,53$, $54,58,69,70,78$, 79, 80, 81, 106, $109,114,119$

Tripolitaine25, 28, 29, $30,31,32,66,97$. 113,131

Tsawa ............. 39, 49

Tunisie.4, 12, 17, 39, $46,51,52,67,102$

Waddaï .................. 30

Wadi Ajal ....... 26, 28

Wadi Al Haya 26, 27, $32,68,73,83,89$, $96,97,98,101$, $103,105,112,139$, $141,142,151,152$, 160

Wadi Ash Shati....27, $28,32,68,96,142$ Zwila ............. 28, 30 


\section{BIBLIOGRAPHIE}

Sont indiqués par* les ouvrages utiles par la diversité et la richesse des articles regroupés.

\section{Libye}

ABDULLATIF A.A., The making of modern Libya, State Formation, Colonization and resistance, 1830-1932, Albany, State University Press of New-York Press, 1994, 222 p.

AIT OUMEZIANE LEDUC A., La recherche d'un équilibre régional en Libye, thèse de doctorat en géographie, Université de Paris I.

ALAWAR M.A., An examination of agricultural development projects in the Fezzan region, Libya, Ph.D. of Geography, University of Northern Colorado, 1979, $132 \mathrm{p}$.

ALAWAR M.A., « Fezzan : population, development and economic change «, in J.A. ALLAN (dir.), Libya since Independance, economic and political development, New-York, Saint Martin's Press, 1982, pp.91-109.

*ALLAN J.A. (dir.), Libya since Independance, economic and political development, New-York, Saint Martin's Press, 1982, 187 p.

*ALLAN J.A., Mc LACHLAN K.S., BURU M. (dir.), Libya, State and region. A study of regional evolution, London, School of Oriental and African Studies, Center of Near and Middle Eastern Studies, 1989, 208 p.

ATTIR M. O., «Social adjustment in new libyan agricultural towns», Ekistics, n³13, 1985, pp.320-326.

BERTARELLI L.V., Libia, Guida del Touring Club Italiano, Milan, Consociazione Turistica Italiano, 1937, $451 \mathrm{p}$.

BISSON J., « La Libye entre clientélisme et régionalisme tribal », Bulletin de l'Association des Géographes Français, 1997, 1, pp.70-82.

BISSON D., BISSON J., FONTAINE J., La Libye. A la découverte d'un pays, Paris, L'Harmattan, 1999, 2 t. (t. 1, Identité libyenne, 196 p. ; t.2, Itinéraires, 358 p.).

BLEUCHOT H., Chroniques et documents libyens, 1969-1980, Paris, CNRS, 1983, 274 p.

BOCCO R., «L'Etat «contourné» en Libye : tribalisme, clientélisme et révolution dans une économie pétrolière », Revue Genève-Afrique, Genève, IUED, vol. XXVI, n², 1988, pp.133-143. 
BORHAM A., FIKRY M., «Le phénomène urbain en Libye, problèmes juridiques et sociaux », Annuaire de l'Afrique du Nord, Paris, CNRS, vol. XI, 1972, pp.79-103.

BULQAMA H.H., QEZEIRI S., La Jamahiriya, une étude géographique, Sirte, 1995, 742 p. (en langue arabe).

BURGAT F., LARONDE A., La Libye, Que-sais-je, Paris, PUF, 1éd., 1996, $128 \mathrm{p}$.

CAUNEILlE A., «Les groupes d'Ouenzerik, tribu du Fezzân », Bulletin de Liaison Saharienne, n³8, 1960, pp.161-173.

CHANSON-JABEUR C., GALLET D., LARONDE A., LOCHON C. (Eds), Aux rivages des Syrtes : La Libye, espace et développement, de l'Antiquité à nos jours, Paris, CHEAM, 2000, 153 p.

*CHRONIQUES et documents de l'Annuaire de l'Afrique du Nord, notamment les articles de H. Bleuchot, F. Burgat, M. Djaziri, L. Martinez, T. Monastiri, 1962-1998, Paris, CNRS.

CLANET J.C., «L'émigration temporaire des Toubou du Kanem vers la Libye », Cahiers géographiques de Rouen, n¹5, 1981, pp.17-33.

CRESTI F., « Projet social et aménagement du territoire dans la colonisation démographique de la Libye (1938-1940) », Correspondances, Tunis, Institut de Recherche sur le Maghreb Contemporain, 1999, pp.11-19.

DAVIS J., Le système libyen. Les tribus et la révolution, Paris, PUF, 1990, 293 p.

DESPOIS J., Géographie humaine, Mission scientifique au Fezzan (1944-1945), Alger, Institut de Recherches Sahariennes, 1946, 260 p.

DIRECTION des TERRITOIRES du SUD, «Le Fezzan », in Les territoires du sud de l'Algérie, Compte rendu de l'œuvre accomplie de 1947 à 1952, Alger, Imprimerie officielle, 1953, pp.21-23.

DUVEYRIER H., Les Touaregs du Nord, Paris, Guallamel Ainé ed., 1864, 499 p.

ELDBLOM L., Structure foncière, organisation et structure sociale. Une étude comparative sur la vie socio-économique dans les trois oasis libyennes de Ghat, Mourzouq et plus particulièrement Ghadamès, Lund, 1968, 428 p.+ planche h.t.

FONTAINE J., «La Libye, un désert côtier riche en hydrocarbures... et en eau ? », Annales de Géographie, n589, 1996, pp.279-295.

FONTAINE J., «Libye, les premiers résultats du recensement de 1995 », L'information Géographique, n¹, 1998, pp.34-39. 
FONTAINE J., «Les mutations de la population libyenne de l'Indépendance à aujourd'hui » in C. Chanson-Jabeur, D. Gallet, A. Laronde, C. Lochon (Eds), Aux rivages des Syrtes : La Libye, espace et développement, de l'Antiquité à nos jours, Paris, CHEAM, 2000, pp.111-133.

GODARD J., « Témoignages sur les derniers épisodes de la présence française au Sahara », Histoire et Défense, n¹7, 1988-1, pp.47-62.

HANDIRI (el) S. A., Commerces et commerçants dans le Sahara central, les échanges entre la Vilayet de Tripoli et les pays de l'Afrique centrale de 1835 à 1911, thèse de doctorat en histoire, Aix-Marseille I, 1996, $434 \mathrm{f}$.

HONGROIS M., Antique Ghadamès, une rose des sables scintillait, Luçon, Hécate, 1987, 154 p.

*JOFFE E.G.H., Mc LACHLAN K.S. (dir.), Social and Economic Development of Libya, Middle East and North Africa Studies Association, London, Wisbech, 1982, 382 p.

LAWLESS R., «Les développements récents de l'agriculture en Libye », Méditerranée, Aix-en-Provence, n¹, 1976, pp.59-69.

LAWLESS R., QEZEIRI S., «Spatial aspects of population change in Libya », Méditerranée, Aix-en- Provence, 1983, n4, pp.81-86.

LAWLESS R., QEZEIRI S., «Libyan urbanization, the role of the small towns » in P. Signoles (dir.), Petites villes et villes moyennes dans le Monde arabe, Fascicule de recherche n¹6-17, Tours, URBAMA, 1986, pp.653-673.

LETHIELLEUX J., Le Fezzan, ses jardins, ses palmiers, Tunis, Institut des Belles Lettres Arabes, 1948, 253 p.

MARTEL A., «Le commerce du natron au Fezzan (1895 - 1899) », Travaux de l'Institut de Recherches Sahariennes, T. XX, 1961, pp.225 - 236.

MARTEL A., La Libye, 1835-1990, Essai de géopolitique historique, Paris, PUF, 1991, 293 p.

MARTINEZ L., « Transformations économiques et mutations politiques sous l'embargo », Annuaire d'Afrique du Nord, Paris, CNRS, vol. XXXVII, 1998, pp.205-229.

MARTINEZ L., coord., La Libye après l'embargo, monde arabe - Maghreb Machrek, Paris, La documentation Française, octobre-décembre 2000, pp.3-65.

MEHESHI (el) A., The transformation of libyan agriculture : the impact of oil revenues on agriculture of a developing country, Ph.D. of Geography, University of California, 1980, 156 p.

MEYNIER O., La pacification du Sahara et la pénétration saharienne (1852 1930), in Cahiers du Centenaire de l'Algérie, II, Alger, 1830, 64 p. 
MIEGE J.L., «La Libye et le commerce transsaharien au XIX ${ }^{\text {ème }}$ siècle », Revue de l'Occident Méditerranéen et de la Méditerranée, $1^{\text {er }}$ sem., 1975, pp.135-168.

MIOSSEC J.M., «La Libye, désert en bord de mer », in F. Durand-Dastès, G. Mutin, dir., Géographie Universelle, Afrique du Nord, Moyen-Orient, Monde Indien, Paris, Belin-Reclus, 1995, pp.119-5123.

NIBLOCK T. , "Pariah» states and sanctions in the Middle East, Iraq, Libya, Soudan, London, Roulder, 2001, 241 p.

PELT A., Libya Independence and the United Nations, a case of planned decolonisation, New Haven - London, The Carnegie endowment for international peace, $1970,1016 \mathrm{p}$.

QEZEIRI S., "L'explosion urbaine en Libye: la contribution des petites villes », in P. Signoles (dir.), Petites villes et villes moyennes dans le Monde arabe, Fascicule de recherche n $16-17$, Tours, URBAMA, 1986, pp.653-673.

QEZEIRI S., " The role of the state and the development of urban centres in Libya « in J.A. Allan, K.S. Mc Lachlan, M. Buru (dir.), Libya, State and region. A study of regional evolution, London, School of Oriental and African Studies, Center of Near and Middle Eastern Studies, 1989, pp.151-171.

RAHUMA S., La politique anglo-française en Libye pendant la période transitoire à l'Indépendance (1943-1951), thèse de doctorat d'histoire, U. de Paris VII, 1995,

SCARIN E., Le oasi del fezzan, Ricerche ed osservazioni di geografia umana, Bologna, N. Zanicchelli ed., vol. 1, 1934.

Secretariat d'Etat au plan, Atlas national de la Jamahiriya, Tripoli, 1979, 118 p. (langue arabe).

Secretariat d'Etat au plan, Recensement agricole de la Jamahiriya, Tripoli, 1987, (langue arabe).

Secretariat d'Etat au plan, Recensements généraux de la population, Tripoli, 1954, 1964, 1973, 1984, 1995, (langue arabe).

Secretariat Général du Gouvernement, Situation économique et politique des anciennes colonies italiennes, $2^{\text {ème }}$ partie, Libye, Notes et Etudes Documentaires $\mathrm{n}^{\circ} 1026$, Série internationale, Paris, La Documentation Française, 1948, 32 p.

Secretariat Général du Gouvernement, Le Fezzan, Notes et Etudes Documentaires $n^{\circ} 936$, Série France d'Outre-Mer, Paris, La Documentation Française, 1948, $11 \mathrm{p}$. 
Secretariat Général du Gouvernement, Situation économique de la Libye, Notes et Etudes Documentaires $n^{\circ} 1765$, Série Economique et Financière, Paris, La Documentation Française, 1953, 24 p.

SOAVE P., Fezzan, il deserto conteso (1842 - 1921), Milan, A. Giuffre ed., 2001, $530 \mathrm{p}$.

SOURIAU C., Libye, l'économie des femmes, Aix-en-Provence, CNRS-CRESM, 1984, 254p.

THIRY J., Le Sahara libyen dans l'Afrique du Nord médievale, Leuven, Peeters Press - Department of Oriental Studies, 1995, 604 p.

TRIAUD J.L., La légende noire de la Senoussiya, une confrérie musulmane sous le regard français (1840-1930), Paris, Maison des Sciences de l'Homme, 1995, 2 t., 1151 p.

VANDEWALLE D., «The failure of liberalization in the Jamahiriya «, in D. Vandewalle (ed.), Kadhafi's Libya, 1969-1994, New-York-St Martin's Press, 1995, pp.203-221.

VANDEWALLE D., Libya since Independence, oil and state building, London, IB Tauris, 1998, 226 p.

WRIGHT J., Libya, Tchad and the Central Sahara, London, Hurst and Co, $168 \mathrm{p}$.

\section{Sahara}

ABABSA S., «L'oasis, une réalité et un concept pour un développement multidimensionnel durable », fascicule multigraphié, 1997, pp.11-12.

BADUEL P.R., Société et émigration temporaire au Nefzaoua, Paris, CNRS, 1980, $121 \mathrm{p}$.

BADUEL P.R., « Emigration et micro-urbanisation dans le Sud tunisien », in Les travailleurs émigrés et le changement urbain des pays d'origine, Etudes méditerranéennes, Poitiers, Fascicule n4, 1983, pp.76-93.

*BADUEL P.R. (dir.), Enjeux sahariens, Paris, CNRS, 1984, 484 p.

BISSON J., «De la Zaouiia à la ville : El Abiod Sidi Cheikh ou la naissance d'une ville nomade » in P. Signoles (dir.), Petites villes et villes moyennes dans le Monde arabe, Fascicule de recherche $\mathrm{n}^{\circ} 16-17$, Tours, URBAMA, 1986, pp.139-152.

*BISSON J. (dir.), Le nomade, l'oasis et la ville, Fascicule de recherche $\mathrm{n}^{\circ} 20$, Tours, URBAMA, 1989, 288 p. 
BISSON J., «Un front pionnier au Sahara tunisien, le Nefzaoua », in J. Bisson (dir.), Campagnes maghrébines, Bulletin de l'Association des Géographes Français, nº 4, 1991, pp. 299-309.

BISSON J., «Le Sahara dans le développement des Etats maghrébins », I et II, Monde Arabe/Maghreb Machrek, Paris, la Documentation Française, $n^{\circ} 134$, 1991, pp. 3-27 et n 135,1992 , pp. 79-106.

BISSON J., Développement et mutations au Sahara maghrébin, Académie d'Orléans-Tours, Centre Régional de Documentation Pédagogique, 24 diapositives, 1993, $172 \mathrm{p}$.

*BISSON J. (coord.), Du Sahara tunisien aux rivages de l'Atlantique, Les Cahiers d'URBAMA, Tours, n², 1993, $161 \mathrm{p}$.

BISSON J., «Le Sahara entre explorations et indépendances », in J. Fontaine (coord.), Sociétés sahariennes entre mythes et développement, Les Cahiers d'URBAMA, Tours-Besançon, n¹2, 1996, pp.45-62.

BISSON J., «Paysanneries du Sahara maghrébin, dynamiques locales et politiques de développement», in J. Fontaine (coord.), Sociétés sahariennes entre mythes et développement, Les Cahiers d'URBAMA, Tours-Besançon, n¹2, 1996, pp.63-80.

BLIN L., «Economie et mobilité dans la Wilaya de Tamanghasset», in $\mathrm{J}$. Bisson (dir.), Le nomade, l'oasis et la ville, Fascicule de recherche $\mathrm{n}^{\circ} 20$, Tours, URBAMA, 1989, pp.91-100.

BOILLEY P.,Les Touaregs Kel Adagh : Dépendances et Révoltes : du Soudan français au Mali contemporain, Paris, Khartala, 1999, 644 p.

BOUDIAF N., «El Oued», Revue Habitat, Tradition et Modernité, Alger, n², 1994, pp. 181-185.

BOURGEOT A., Les sociétés touarègues, nomadisme, identités, résistances, Paris, Khartala, 1995, 544 p.

CAPOT-REY R., Le Sahara Français, Paris, PUF, 1953, 564 p.

CAPOT-REY R., «Les palmeraies du Tibesti » in Maghreb et Sahara : études géographiques offertes à Jean Despois, 1973, pp.69-78.

CARATINI S., Les Rgaybat (1610-1934), Paris, L'Harmattan, 1989, 2 tomes (t.1, Des chameliers à la conquête d'un territoire, 290 p.; t.2, Territoire et société, 290 p.).

CLAUDOT HAWAD H., «La conquête du «vide » ou la nécessité d'être nomade chez les Touaregs », in J. Fontaine (coord.), Sociétés sahariennes entre mythes et développement, Les Cahiers d'URBAMA, Tours-Besançon, $\mathrm{n}^{\circ} 12,1996, \mathrm{pp} .101-112$. 
*CLOUET Y., DOLLE V. (coord.), $\mathrm{N}^{\circ}$ spécial : Les oasis, Sécheresse, sciences et changements planétaires, Montrouge, AUPELF, vol.9, $\mathrm{n}^{\circ} 2,1998$, pp.79-174.

COTE M., «Espoirs et menaces sur le Sahara : les formes récentes de mise en valeur agricole », in J. Bisson (coord.), Du Sahara tunisien aux rivages de l'Atlantique, Les Cahiers d'URBAMA, Tours, n8, 1993, pp.11-27.

DESIRE-VUILLEMIN G., « Les villes sahariennes dans leur évolution et leur rôle », in A.M. Frérot (dir.) Espaces et sociétés en Mauritanie, Fascicule de recherche $\mathrm{n}^{\circ} 33$, Tours, URBAMA, 1998, pp.67-76.

DUVIGNAUD J., Chebika, suivi de retour à Chebika : changements dans un village du Sud tunisien, Paris, Plon, 1991, 501 p.

*FONTAINE J. (coord.), Sociétés sahariennes entre mythes et développement, Les Cahiers d'URBAMA, Tours - Besançon, n¹2, 1996, 151 p.

FONTAINE J., «Les populations sahariennes », in J. Fontaine (coord.), Sociétés sahariennes entre mythes et développement, Les Cahiers d'URBAMA, Tours - Besançon, n²12, 1996, pp.33-44.

GREGOIRE E., « Réseaux de commerce et maillages urbains », in E. Le Bris (coord.), Villes du Sud, sur la route d'Istanbul, Paris, ORSTOM, pp.147-165.

GREGOIRE E., «Sahara nigérien : terre d'échanges », in J. Egg, J. Herrera (ed.), Echanges transfrontaliers et intégration régionale en Afrique subsaharienne, Paris, ORSTOM, pp.147-165.

GREGOIRE E., Touaregs du Niger, le destin d'un mythe, Paris, Khartala, 1999, $339 \mathrm{p}$.

KASSAH A., «Tozeur et son oasis : problèmes d'aménagement d'une ville oasienne », Les Cahiers d'URBAMA, Tours, n8, 1993, pp. 51-75.

LE CLEZIO J. et J.M.G., Gens des nuages, Paris, Stock, 1997, 120 p.

MONOD T., «Les bases d'une division géographique du domaine saharien », Bulletin de l'I.F.A.N., Tome XXX, sér. B, n¹, 1968, pp. 269-288.

NACIRI M., «Les ksouriens sur la route. Emigration et mutation spatiale de l'oasis de Tinjdad », Annuaire de l'Afrique du Nord, Paris, CNRS, Tome XXV, 1986, pp.347-364.

*RETAILLE D. (coord.), Etudes Sahariennes, Cahiers Géographiques de Rouen, Université de Rouen, $\mathrm{n}^{\circ} 26,1986,82$ p.

RETAILLE D., «La conception nomade de la ville », in J. Bisson (dir.), Le nomade, l'oasis et la ville, Fascicule de recherche $n^{\circ} 20$, Tours, URBAMA, 1989, pp.21-35. 
RETAILLE D., «Sécheresse, migration et aménagement du territoire en Mauritanie », Revue de Géographie de Lyon, vol.70, n³-4, 1995, pp.233-238.

SOUAMI T., L'institutionnel face à l'espace autoproduit. Histoires d'une possible co-production de la ville. L'exemple du sud de l'Algérie, thèse de doctorat en géographie, Université de Paris 8, 1999, 742 p.

VILLASANTE-DE BEAUVAIS M., «Constructions et redéfinitions identitaires en Mauritanie », in M. Lussault, P. Signoles (dir.), La citadinité en questions, Fascicule de recherche n²9, Tours, URBAMA, 1996, pp.83-96.

ZINE A., «Les ksour », Revue Habitat, Tradition et Modernité, Alger, n², 1994, pp.17-22.

\section{Références théoriques}

ARRIF A., «Variations spatiales du public et du privé à travers les exemples de Ben M'sik et de Hay Moulay Rachid à Casablanca », Les Cahiers d'URBAMA, Tours, n 13, 1997, pp.61-89.

*BADUEL P.R., « La production de l'espace national au Maghreb », in P.R. Baduel (dir.), Etats, territoires et terroirs au Maghreb, Paris, CNRS, 1985, pp.3-39.

BADUEL P.R., «Le territoire d'Etat entre imposition et subversion: exemples saharo-sahéliens », in B. Badie et M.-C. Smouts (dir.), L'international sans territoire, Cultures et conflits, $\mathrm{n}^{\circ} 21-22$, printemps/été, 1996, pp.41-74.

BAZIN M., «L'urbanisation des campagnes en Turquie : l'exemple de Sulusaray », Annales de Géographie, n575, 1994, pp.41-56.

BENNAFLA K., «Entre Afrique noire et Monde arabe, nouvelles tendances des échanges informels tchadiens », Revue Tiers Monde, n²152, t.38, 1997, pp. 879-896.

BOUMAZA N., Questions de mutations et de formes urbaines au Maghreb, Grenoble, fascicule dactylographié, 1993.

CERTEAU (de) M., L'invention $d u$ quotidien, t.1, Arts de faire, coll. folio-essai, Paris Gallimard, éd. de 1990, 350 p.

COTE M., L'Algérie ou l'espace retourné, Paris, Flammarion, 1988, 362 p.

COTE M., «Algérie, la quête de l'autonomie locale », in M. Lavergne (dir.), Monde arabe, le retour du local, Peuples Méditerranéens, n72-73, 1995, pp.123-132.

DAMETTE F., «La question urbaine dans le Monde arabe », Espaces et sociétés n65, semestriel, n², L’Harmattan, 1991, pp.137-144. 
DI MEO G., Géographie sociale et territoires, Paris, Nathan Université, 1998, $317 \mathrm{p}$.

DI MEO G., "Géographies tranquilles du quotidien, une analyse à la contribution des sciences sociales et de la géographie à l'étude des pratiques spatiales », Cahiers de Géographie du Québec, vol. 43, n 118, avril 1999, pp.75-93.

ESCALLIER R., «Ville et informalité dans le Monde arabe », Les Cahiers de la Méditerranée, n56, 1998, pp.39-85.

*LAVERGNE M, TROIN J.F. (coord.), «Villes petites et moyennes au Moyen-Orient », Annales de Géographie, n575, 1994, pp.3-4.

*LAVERGNE M. (dir.), Monde arabe, le retour du local, Peuples Méditerranéens, nº72-73, 1995, $337 \mathrm{p}$.

LEVY J., L'espace légitime, Paris, Presses de la Fondation Nationale des Sciences Politiques, 1994, 442 p.

LEVY J., Europe, une géographie, Paris, Hachette, 1997, 288 p.

LEVY J., Le tournant géographique, coll. Mappemonde, Paris, Belin, 1999, $399 \mathrm{p}$.

LOUISET O., 2000, «L'urbanité ailleurs », in J. Lévy, M. Lussault, Logiques de l'espace, esprit des lieux, Mappemonde, Belin, pp.157-164.

LUSSAULT M., SIGNOLES P. (dir.), La citadinité en questions, Fascicule de recherche $\mathrm{n}^{\circ} 29$, Tours, URBAMA, 1996, $157 \mathrm{p}$.

MBEMBE A., «Vers une nouvelle géopolitique africaine », in Afriques en renaissance, Manière de voir n ${ }^{\circ}$ 1, Mai-Juin 2000, pp.10-15.

MIOSSEC J.M., «Urbanisation des campagnes et ruralisation des villes en Tunisie », Annales de Géographie, n521, 1985, pp.38-62.

NAVEZ-BOUCHANINE F., "Modèles d'habiter et crise de l'urbain: la situation vue à partir du Maroc », Espaces et sociétés nº5, semestriel n², L'Harmattan, 1991, pp.85-108.

PETONNET C., «L 'anonymat urbain », in C. Ghorra-Gobin, Penser la ville de demain. Qu'est-ce qui institue la ville? coll. Géographies et cultures, Paris, L'Harmattan, 1994, pp.17-21.

PONS F.X., «Le Sahara et ses frontières étatiques : géopolitiques et enjeux », L’Information Géographique, n4, 1997, pp.154-159.

PORTES A., «La mondialisation par le bas, l'émergence des communautés transnationales », Actes de la Recherche en Sciences Sociales, 1999, n 129 , pp.15-25. 
RETAILLE D., Le monde du géographe, Paris, Presses de Sciences-Po, 1997, $285 \mathrm{p}$.

SAUTTER G., «Réflexions sur les «petites villes » dans les pays en voie de développement », in P. Vennetier (dir.), Problèmes de la croissance urbaine dans le monde tropical, Travaux et Documents du CEGET, n45, 1982, pp.393-420.

SIDI BOUMEDINE R., «La citadinité, une notion impossible », in M. Lussault, P. Signoles (dir.), La citadinité en questions, Fascicule de recherche n²9, Tours, URBAMA, 1996, pp.49-56.

SIGNOLES P., «Quelques réflexions sur les petites villes et les villes moyennes dans le Monde arabe », in P. Signoles (dir.), Petites villes et villes moyennes dans le Monde arabe, Fascicule de recherche $\mathrm{n}^{\circ} 16-17$, Tours, URBAMA, 1986, pp.811-830.

*SIGNOLES P. (dir.), Petites villes et villes moyennes dans le Monde arabe, Fascicule de recherche ${ }^{\circ} 16-17$, Tours, URBAMA, 1986, 833 p.

*SIGNOLES P., KADI (el) G., SIDI BOUMEDINE R. (dir.), L'urbain dans le Monde arabe, Politiques, instruments et acteurs, Paris, IREMAM-CNRS, 1999, $373 \mathrm{p}$. 


\section{RESSOURCES CARTOGRAPHIQUES}

Au Centre des Archives d'Outre-Mer (CAOM, Aix-en-Provence)

\begin{tabular}{|c|c|c|c|c|c|}
\hline Code & Cote & Titre & Date & Echelle & Commentaire \\
\hline \multicolumn{6}{|c|}{ Série Archives d'Outre-Mer (zone générique cp) } \\
\hline 00096 & 2PL0051 & Nord Sahara & 1889 & $1 / 2.000 .000$ & $\begin{array}{l}\text { Plan de Tripoli et } \\
\text { Ghadamès }\end{array}$ \\
\hline 00198 & 2PL00137 & Afrique-Fezzan & 1952 & $1 / 2.000 .000$ & Géologie \\
\hline 00200 & 2PL00139 & Sahara & 1959 & $1 / 2.000 .000$ & Hydrocarbures \\
\hline 00208 & 1PL00069 & \begin{tabular}{|l|} 
Confins \\
algéro-tripolitains
\end{tabular} & 1912 & $1 / 1.000 .000$ & \\
\hline 00261 & 2PL00165 & Sahara & 1961 & $1 / 2.000 .000$ & Hydrocarbures \\
\hline 00965 & 2PL00514 & Régence de Tripoli & 1850 & $1 / 2.000 .000$ & Routes sahariennes \\
\hline 01208 & 1PL00695 & Tripolitaine & 1911 & $1 / 200.000$ & \\
\hline 01209 & 1PL00696 & Tripolitaine & 1911 & $1 / 200.000$ & \\
\hline 01210 & 1PL00697 & Tripolitaine & 1911 & $1 / 200.000$ & \\
\hline 01211 & 1PL00698 & Koufra & 1923 & $1 / 400.000$ & \\
\hline \multirow[t]{4}{*}{01212} & 1PL00699 & Benghazi & 1924 & $1 / 25.000$ & \\
\hline & B151/6 & Tripoli & 1893 & $1 / 2.000 .000$ & \\
\hline & B151/7 & Benghazi & 1893 & $1 / 2.000 .000$ & \\
\hline & B151/12 & Mourzouq & 1893 & $1 / 2.000 .000$ & \\
\hline \multirow[t]{7}{*}{ CC3 } & & Fezzan & 1946 & $1 / 2.000 .000$ & \\
\hline & IGN143 & \begin{tabular}{|l} 
Afrique \\
Nord-Ouest \\
\end{tabular} & & $1 / 2.000 .000$ & \\
\hline & IGN362 & Pistes et routes & & $1 / 2.000 .000$ & \\
\hline & IGN158 & Koufra & & $1 / 1.000 .000$ & \\
\hline & IGN178 & Mourzouq & & $1 / 1.000 .000$ & \\
\hline & IGN150 & Benghazi & & $1 / 1.000 .000$ & \\
\hline & IGN195 & Tripoli & & $1 / 1.000 .000$ & \\
\hline \multicolumn{6}{|c|}{ Série Service Géographique des Colonies (zone générique fm) } \\
\hline & \begin{tabular}{|l} 
SGEOCO \\
L26
\end{tabular} & Confins libyens & $\begin{array}{l}1927- \\
1957\end{array}$ & & $\begin{array}{l}\text { Croquis des } \\
\text { frontières } \\
\text { libyennes }\end{array}$ \\
\hline
\end{tabular}




\section{Ressources diverses}

- Cartes soviétiques au $1 / 200000^{\text {ème }}$ (publiées durant les années 1970-1980), couverture intégrale de la Libye.

- $\quad$ Photocopies de coupures au 1/25 $000^{\text {ème }}$ (publiées durant les années 1980), Brak, Sebha, Oubari, al Gharayfa-Germa.

Photocopies de photographies aériennes, 1981, al Gharayfa, Germa, Oubari.

- $\quad$ Photocopie du Plan d'aménagement 2000, ville de Sebha.

- $\quad$ Echantillons Images SPOT, 1998-1999, couverture du Fezzan.

- $\quad$ Images Landsat, 1984-1985, Mourzouq, Oubari, Sebha. 


\section{TABLE DES MATIÈRES}

\section{REMERCIEMENTS 2}

\section{UNE LECTURE URBAINE DU FEZZAN 3}

VERS L'URBANISATION TOTALE DU SAHARA 4 Un état des lieux 4 Urbanisation par le haut, urbanisation par le bas 7 Les réseaux de circulation produisent de l'urbain 9 LECTURES ET RELECTURES GÉOGRAPHIQUES DU SAHARA 14 Une géographie saharienne rurale et fractionnée par les aires culturelles 14

Une entrée urbaine sur le Fezzan contemporain 18

\section{Première Partie 22}

\section{LES VILLES DU FEZZAN ENTRE TERRITOIRE D'ETAT ET CARREFOUR 22}

CHAPITRE 1 - LE FEZZAN, UN CARREFOUR SAHARIEN 24 Les échelle du carrefour fezzanais 25 Hinterland ou périphérie? Les enjeux coloniaux (1850 - 1950) 29

CHAPITRE 2 - L'INTEGRATION DU FEZZAN AU TERRITOIRE NATIONAL 34

Désenclavement et mise en place de la trame urbaine 35 L'urbanisation généralisée, une dynamique en deux temps 38 Les migrations internes, produits et ressorts de la croissance urbaine 42

CHAPITRE 3 - LE RENOUVEAU DU CARREFOUR FEZZANAIS 46 Un espace migratoire de proximité 46 Un « entre-deux » entre l'Afrique et le Maghreb 50 Les échelles du nouveau carrefour 54

LES CYCLES URBAINS DU FEZZAN 60

\section{DEUXIÈME PARTIE 62}

\section{DES DYNAMIQUES LOCALES ENCADRÉES PAR L'ETAT 62}

CHAPITRE 4 - TRANSACTIONS ENTRE LE NATIONAL ET LE LOCAL 64 L'omniprésence de l'Etat sans Etat 64

De l'Infitah (ouverture) à la mutation de l'économie locale 68 
Des acteurs entre cadre familial et étatique 73

Ghadamés, entre local et global 77

CHAPITRE 5 - LA FIN DU MODÈLE URBAIN ÉTATIQUE 83

Les étapes de la croissance 83

Un modèle d'évolution commun? 88

CHAPITRE 6 - LA MUTATION RURALE SOUS L'INFLUENCE DE

L'URBAIN 95

Crise et mutation d'un espace oasien 95

Les aléas de l'agriculture bureaucratique 98

L'essor de l'agriculture privée périurbaine 101

Les complémentarités entre l'urbain et le rural 106

AGIR SUR L'ESPACE À L'HEURE DE L'INFITAH LIBYENNE 108

\section{TROISIÈME PARTIE 110}

\section{VERS UNE URBANITÉ SAHARIENNE 110}

CHAPITRE 7 - DE LA VILLE PENSÉE À LA VILLE VÉCUE 113

La conception d'un modèle d'urbanisation 113

Crise et réappropriation du modèle importé 116

Le logement, indice de changement social 120

CHAPITRE 8 - SEBHA, FACETTES DU COSMOPOLITISME SAHARIEN 128

La planification urbaine à l'épreuve des migrations 128

Le quartier, une unité familiale 131

Des centres créés par les usagers de la ville 134

CHAPITRE 9 - L'URBANITÉ SANS VILLE DU WADI Al HAYA 139

Consternants paysages urbains! 139

Visions et discours des habitants du Wadi 141

Territorialiser par les pratiques routinières et quotidiennes 146

Les limites d'une urbanité idéale 148

UNE URBANITÉ, DES URBANITÉS 151

CONCLUSION GÉNÉRALE : QU'EST-CE QUE L'URBAIN DANS LE

SAHARA LIBYEN ? 153

«Haut» et « bas », national et local, des ruptures à nuancer 156

Le temps long du réseau et le temps court du territoire d'Etat 159

La portée d'un fait spatial : l'urbanisation généralisée 160

TABLE DES ILLUSTRATIONS 164

TABLE DES TABLEAUX 164

TABLE DES PHOTOGRAPHIES 164 
INDEX DES NOMS DE LIEUX 165

BIBLIOGRAPHIE 167

RESSOURCES CARTOGRAPHIQUES 177

TABLE DES MATIÈRES 179 